Leitlinien

Z Rheumatol 2019· 78 (Suppl 1):S3-S64 https://doi.org/10.1007/s00393-019-0670-3

(c) Deutsche Gesellschaft für Rheumatologie e.V. Published by Springer Medizin Verlag $\mathrm{GmbH}$. All rights reserved 2019
U. Kiltz' für DGRh · J. Braun' für DGRh · A. Becker ${ }^{2}$ für DEGAM - J.-F. Chenot ${ }^{3}$ für DEGAM - M. Dreimann für DWG - L. Hammel ${ }^{5}$ für DVMB · A. Heiligenhaus ${ }^{6}$ für DOG · K.-G. Hermann ${ }^{7}$ für DRG · R. Klett ${ }^{8}$ für DGMM - D. Krause ${ }^{9}$ für DGRh · K.-F. Kreitner ${ }^{10}$ für DRG • U. Lange ${ }^{11}$ für DGPMR/DGRW · A. Lauterbach ${ }^{12}$ für Physio Deutschland $\cdot$ W. Mau ${ }^{13}$ für DGPMR/DGRW $\cdot$ R. Mössner ${ }^{14}$ für DDG $\cdot$ U. Oberschelp ${ }^{15}$ für DGOOC · S. Philipp ${ }^{16}$ für DDG - U. Pleyer ${ }^{17}$ für DOG · M. Rudwaleit ${ }^{18}$ für DGRh . E. Schneider ${ }^{19}$ für DGOOC · T. L. Schulte ${ }^{20}$ für DWG - J. Sieper ${ }^{21}$ für DGRh . A. Stallmach ${ }^{22}$ für DGIM • B. Swoboda ${ }^{23}$ für DGOOC/DGORh · M. Winking ${ }^{24}$ für DGNC ${ }^{1}$ Rheumazentrum Ruhrgebiet, Ruhr-Universität Bochum, Herne, Deutschland; ${ }^{2}$ Allgemeinmedizin, präventive und rehabilitative Medizin, Universität Marburg, Marburg, Deutschland; ${ }^{3}$ Universitätsmedizin Greifswald, Greifswald, Deutschland; ${ }^{4}$ Zentrum für Operative Medizin, Klinik und Poliklinik für Unfall-, Hand- und Wiederherstellungschirurgie, Universitätsklinikum Hamburg-Eppendorf (UKE), Hamburg, Deutschland; ${ }^{5}$ Geschäftsstelle des Bundesverbandes der DVMB, Schweinfurt, Deutschland; ${ }^{6}$ Augenzentrum und Uveitis-Zentrum, St. Franziskus Hospital, Münster, Deutschland; ${ }^{7}$ Institut für Radiologie, Charité Berlin, Berlin, Deutschland; ${ }^{8}$ Praxis Manuelle \& Osteopathische Medizin, Langgöns, Deutschland; ${ }^{9}$ Gladbeck, Deutschland; ${ }^{10} \mathrm{Klinik}$ und Poliklinik für Diagnostische und Interventionelle Radiologie, Universitätsmedizin Mainz, Mainz, Deutschland; "Kerckhoff-Klinik, Rheumazentrum, Osteologie \& Physikalische Medizin, Bad Nauheim, Deutschland; ${ }^{12}$ Schule für Physiotherapie, Orthopädische Universitätsklinik Friedrichsheim, Frankfurt, Deutschland; ${ }^{13}$ Institut für Rehabilitationsmedizin, Medizinische Fakultät, Martin-Luther-Universität Halle-Wittenberg, Halle (Saale), Deutschland; ${ }^{14}$ Klinik für Dermatologie, Universitätsmedizin Göttingen, Göttingen, Deutschland; ${ }^{15}$ Werne a.d. L., Deutschland; ${ }^{16}$ Praxis für Dermatologie, Oranienburg, Deutschland; ${ }^{17}$ Campus Virchow-Klinikum, Charité Centrum 16, Klinik f. Augenheilkunde, Charité, Berlin, Deutschland; ${ }^{18}$ Klinikum Bielefeld, Bielefeld, Deutschland; ${ }^{19} \mathrm{Abt}$. Fachübergreifende Frührehabilitation und Sportmedizin, St. Antonius Hospital, Eschweiler, Deutschland; ${ }^{20}$ Klinik für Orthopädie und Unfallchirurgie, Orthopädische Universitätsklinik, Ruhr-Universität Bochum, Bochum, Deutschland; ${ }^{21}$ Medizinische Klinik für Gastroenterologie, Infektiologie und Rheumatologie, Charité - Universitätsmedizin Berlin, Berlin, Deutschland; ${ }^{22}$ Klinik für Innere Medizin IV, Universitätsklinikum Jena, Jena, Deutschland; ${ }^{23}$ Abteilung für Orthopädie und Rheumatologie, Orthopädische Universitätsklinik, Malteser Waldkrankenhaus St. Marien, Erlangen, Deutschland; ${ }^{24}$ Zentrum für Wirbelsäulenchirurgie, Klinikum Osnabrück, Osnabrück, Deutschland

\section{Langfassung zur S3-Leitlinie Axiale Spondyloarthritis inklusive Morbus Bechterew und Frühformen, Update 2019}

\section{Evidenzbasierte Leitlinie der Deutschen Gesellschaft für Rheumatologie (DGRh) und der beteiligten medizinisch- wissenschaftlichen Fachgesellschaften und weiterer Organisationen*}

U. Kiltz,J.Braun (federführend)

\title{
*Fachgesellschaften:
}

Deutsche Gesellschaft für Rheumatologie (DGRh) (federführend), Deutsche Gesellschaft für Orthopädie und orthopädische Chirurgie (DGOOC), Deutsche Gesellschaft für orthopädische Rheumatologie (DGORh), Deutsche Dermatologische Gesellschaft (DDG), Deutsche Gesellschaft für Allgemeinmedizin und Familienmedizin (DEGAM), Deutsche Gesellschaft für Innere Medizin (DGIM), Deutsche Gesellschaft für Manuelle Medizin (DGMM), Deutsche Gesellschaft für Neurochirurgie (DGNC), Deutsche ophthalmologische Gesellschaft (DOG), Deutsche Gesellschaft fürPhysikalische Medizin und Rehabilitation (DGPMR), Deutsche Gesellschaft für Rehabilitationswissenschaften (DGRW), Deutsche Röntgengesellschaft (DRG), Deutsche Wirbelsäulengesellschaft(DWG)

Weitere:

Deutsche Vereinigung Morbus Bechterew (DVMB), Physio Deutschland 


\section{Infobox AWMF-Leitlinien \\ Register Nummer: 060/003, Entwicklungsstufe: $\mathrm{S} 3$}

Version: 2018

Leitliniensekretariat: PD Dr. med. Uta Kiltz, Rheumazentrum Ruhrgebiet, Herne Methodische Beratung: Frau Dr. S. Blödt, AWMF, Frau C. Weseloh, DGRh Sie finden Kurzfassung, Langfassung, Patientenleitlinie und den Leitlinienreport auf der Seite der AWMF, unter https://www.awmf. org/leitlinien/detail/II/060-003.html

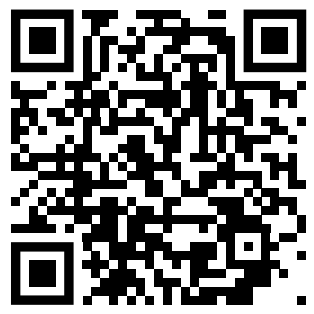

Besonderer Hinweis:

Die Medizin unterliegt einem fortwährenden Entwicklungsprozess, sodass alle Angaben, insbesondere zu therapeutischen Verfahren, immer nur dem Wissenstand zur Zeit der Drucklegung der Leitlinie entsprechen können. Hinsichtlich der angegebenen Empfehlungen zur Therapie wurde die größtmögliche Sorgfalt beachtet.

Der Benutzer selbst bleibt verantwortlich für jede diagnostische und therapeutische Applikation, Medikation und Dosierung.

\section{Zusatzmaterial online}

Die Online-Version dieses Beitrags (https://doi.org/10.1007/s00393-019-0670-3) enthält die Evidenztabellen (Tab. 11, 12, 13, 14).

Beitrag und Zusatzmaterial stehen Ihnen auf www.springermedizin.de zur Verfügung. Bitte geben Sie dort den Beitragstitel in die Suche ein, das Zusatzmaterial finden Sie beim Beitrag unter "Ergänzende Inhalte".

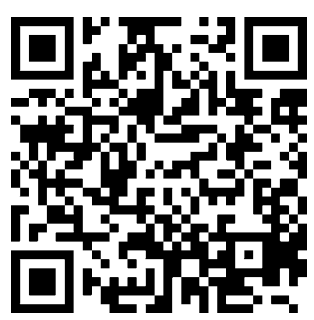

\section{Einleitung/Vorbemerkung}

\subsection{Definition}

Spondyloarthritiden (SpA) sind entzündlich rheumatische Erkrankungen, die durch Entzündungen im Bereich der Wirbelsäule gekennzeichnet sind, welche häufig zu Rückenschmerzen führen. Die gesamte Gruppe der SpA stellt sich heterogen dar mit einigen klinischen und genetischen Gemeinsamkeiten, aber auch mit Überlappungen und Übergängen in verwandte entzündlich rheumatische Erkrankungen. Die Beteiligung des Achsenskeletts und der Sehnenansätze (Enthesen) sowie die Assoziation mit dem MHC-Klasse-IAntigen HLA-B27 ist charakteristisch für diese Erkrankungsentität [1].

Die Gesamtgruppe der SpA lässt sich auf Basis klinischer und z.T. radiologischer Befunde unterteilen in eine prädominant axiale $\mathrm{SpA}$ inklusive des Morbus Bechterew (M. Bechterew $=$ ankylosierende Spondylitis [AS]) und eine prädominant periphere Form. Beider Mehrzahlder Patienten überwiegt eine prädominant axiale Manifestation, d. h. bei den Patienten stehen Schmerzen und Bewegungseinschränkung des Achsenskelettes im Vordergrund. Daneben gibt es aber auch Patienten ohne axiale Symptomatik, die unter einer prädominant peripheren Manifestation wie einer Arthritis, Enthesitis und/oder Daktylitis leiden. Patienten mit einer SpA können zusätzlich unter einer Psoriasis vulgaris, einer anterioren Uveitis und/oder einer chronisch entzündlichen Darmerkrankung (CED) leiden [2, 3].

Die Bezeichnung Morbus Bechterew ist gegenüber der internationalen Bezeichnung AS im deutschen Sprachraum gebräuchlicher. Beide Bezeichnungen werden in der Leitlinie parallel für diejenige Form der axialen SpA (axSpA) verwendet, bei der schon strukturelle Läsionen in den Sakroiliakalgelenken (SI-Gelenke) vorhanden bzw. röntgenologisch sichtbar sind (röntgenologische axiale SpA). Mit der Einführung der Assessment of SpondyloArthritis International Society(ASAS)-Klassifikationskriterien ist die axiale $\mathrm{SpA}$ in die nichtröntgenologische axiale SpA (nr-
axSpA) und die klassische AS (= röntgenologische axSpA) unterteilt worden (s. Abschn. 4.1) [2]. Es handelt sich hierbei aber eher um eine arbiträre Trennung, da es sich um ein einziges Krankheitsbild handelt, d.h. die Unterschiede zwischen der nr-axSpA und der AS bestehen nur hinsichtlich der Krankheitsdauer und hinsichtlich des Ausmaßes der $\mathrm{zu}$ einem bestimmten Zeitpunkt vorhandenen strukturellen Veränderungen. Dementsprechend wird mit dem Terminus nr-axSpA diejenige Patientengruppe beschrieben, bei der (noch) keine eindeutigen strukturellen Läsionen in den SI-Gelenken im konventionellen Röntgenbild zu sehen sind. Die Gruppe nr-axSpA ist nicht mit der früher häufig so genannten Gruppe der undifferenzierten SpA (uSpA) identisch. In dieser Gruppe fanden sich neben Patienten, die heute als nr-axSpA klassifiziert werden, auch Patienten, die unter einer rein peripheren SpA litten.

\subsection{Epidemiologie}

Die Prävalenz der gesamten Gruppe der SpA liegt zwischen 0,4 und $2 \%$ - belastbare Untersuchungen zur Prävalenz in verschiedenen SpA-Gruppen außerhalb der AS existieren nicht $[1,4]$. Ein aktuelles systematisches Review hat eine durchschnittliche AS-Prävalenz pro 10.000 für Europa mit 23,8, für Asien mit 16,7 , für Nordamerika mit 31,9, für Lateinamerika mit 10,2 und für Afrika mit 7,4 berechnet [4]. Die Unterschiede in der Häufigkeit sind regional bedingt und ergeben sich $z$. T. durch die unterschiedliche Häufigkeit von HLA-B27 in der Bevölkerung [1]. Für die AS wird eine Prävalenz weltweit zwischen 0,1 und $1,4 \%$ ermittelt, in Deutschland erscheint eine Prävalenz zwischen 0,3 und 0,5\% wahrscheinlich [5].

Bei der SpA geht man heute von einem Geschlechterverhältnis Männer zu Frauen von zumindest 2:1 aus. Daten der Kerndokumentation des Deutschen Rheuma Forschungs-Zentrums (DRFZ) von 2016 zeigen, dass ca. 61,5\% der Patienten mit M. Bechterew männlich gegenüber 38,5\% weiblichen Patientinnen sind. Das Verhältnis beträgt jedoch 1:1 bei Patienten mit nr-axSpA. 


\subsection{Krankheitsverlauf/Prognose}

Die ersten Symptome einer SpA treten im Durchschnitt im 2. bis 3. Lebensjahrzehnt auf [1]. Patienten mit familiärer Disposition erkranken früher als Patienten mit einer sporadischen Manifestation [6]. Juvenile Manifestationsformen sind mit dem schlechtesten Outcome vergesellschaftet [7]. Anhand der US-amerikanischen Kohorte PSOAS ist beschrieben worden, dass AS-Patienten mit schwarzer Hautfarbe ein schlechteres Outcome haben als AS-Patienten mit weißer Hautfarbe [8]. In einer südamerikanischen Kohorte wurde ein Drittel der Patienten nach dem 50. Lebensjahr symptomatisch und erst im höheren Lebensalter diagnostiziert [9].

In den ersten Jahren stehen Schmerzen an der Wirbelsäule und eine variable extraskeletale Beteiligung im Vordergrund der Beschwerdesymptomatik. Mit zunehmender Dauer der Erkrankung entstehen bei vielen, aber nicht allen Patienten Verknöcherungen am Achsenskelett, die in wenigen Fällen zu der charakteristischen Bambusstabwirbelsäule führen können. Sowohl entzündliche als auch strukturelle Veränderungen führen zu einer Einbuße der Funktionsfähigkeit [10]. Die Einbuße der Funktionsfähigkeit ist nicht allein auf den körperlichen Bereich beschränkt, sondern es liegt auch eine Einschränkung der Lebensqualität, Alltagsaktivität und der Teilhabe (Partizipation) am sozialen Leben vor [11, 12]. Diese Einschränkungen werden durch Umweltfaktoren oder personenbezogene Faktoren beeinflusst (Abschn. 9). Ein Erkrankungsbeginn in jungen Jahren kann bei den Patienten erhebliche sozioökonomische Konsequenzen haben $[13,14]$.

Rückenschmerzen als erstes Frühsymptom der Erkrankung werden bei SpA-Patienten häufig fehlgedeutet, und es kommt zunächst weder zu einer klaren Diagnose noch zu einer effektiven Therapie [15]. Unter den rheumatischen Erkrankungen zeichnet sich die axiale SpA durch eine lange Zeitdauer (meist von mehreren Jahren) zwischen Symptombeginn und Diagnosestellung aus. Dies ist vor allem darauf zurückzuführen, dass nicht ein einzelnes Symptom wegweisend für die Diagnose ist, sondern dass die „richtigen“ Patienten aus der großen Gruppe der Patienten mit unspezifischen Rückenschmerzen möglichst optimal vorselektiert werden müssen. Während bei männlichen Patienten mit AS/M. Bechterew eine Diagnoseverzögerung von 5 bis 10 Jahren ermittelt wurde, müssen Frauen mit einer Verzögerung von bis zu 14 Jahren rechnen [16, 17]. Daten aus europäischen Registern (z. B. DANBIO aus Dänemark) zeigen einen Rückgang der Diagnoseverzögerung auf Monate bis wenige Jahre [18]. Weltweite Daten zeigen jedoch weiterhin eine signifikante Diagnoseverzögerung von mehreren Jahren [19].

Die Ausprägung der klinischen Symptomatik der SpA ist variabel, und es kommen häufig fließende Übergänge zwischen den Subgruppen vor. Bei AS/M. Bechterew-Patienten ist bekannt, dass ca. ein Drittel der Patienten einen schwerwiegenden Verlauf erleben wird [20]. Über den natürlichen Verlauf der Gesamtgruppe der axialen SpA ist nur wenig bekannt, die Mehrzahl der Studien konzentriert sich auf die AS.

\subsection{Begründung der Leitlinie}

Aufgrund des chronischen Verlaufes und der Manifestation der Erkrankung in jungen Lebensjahren hat die axiale SpA eine erhebliche Bedeutung für die Patienten und die Gesellschaft. Durch die oft lange Diagnoseverzögerung kann das Ziel, strukturelle Läsionen am Achsenskelett $\mathrm{zu}$ verhindern, nicht erreicht werden. Zudem konnte in kontrollierten Studien gezeigt werden, dass insbesondere Patienten mit einem kurzen Krankheitsverlauf von der Therapie profitierten (s. Abschn. 8). Um eine frühzeitige Therapie bei den Patienten einleiten zu können, ist eine richtige und zeitnahe Diagnosestellung für die Patienten von essenzieller Bedeutung. Durch den Zuwachs an epidemiologischen Daten sowie neuen Therapieoptionen ergab sich die Notwendigkeit eines Updates der Fassung der Leitlinie von 2013.

\subsection{Ziel/Adressaten der Leitlinie}

Das Ziel der vorliegenden Leitlinie ist, die evidenzbasierte Diagnostik und Therapie der axialen SpA darzustellen und damit den Betroffenen die Möglichkeit einer frühzeitigen Diagnosestellung zu eröffnen und die Einleitung einer wissenschaftlich begründeten Therapie zu ermöglichen. Die Leitlinie soll damit helfen, die medizinische Versorgung dieser Patientengruppe zu verbessern. Dazu soll die Zeit bis zur Diagnosestellung reduziert, eine effektive Therapie rasch eingeleitet, strukturelle Läsionen verhindert, die Versorgung optimiert, die Lebensqualität verbessert und die Arbeitsfähigkeit erhalten werden. Die vorliegende Leitlinie fokussiert auf die axiale SpA inklusive AS/M. Bechterew, und soweit es die Verständlichkeit notwendig macht, werden Aspekte der peripheren SpA, die sich auf die Diagnostik und Therapie der axialen $\mathrm{SpA}$ beziehen, mitberücksichtigt.

Die Leitlinie richtet sich an Ärzte sowie Angehörige nichtärztlicher Berufsgruppen, die an der Versorgung der Patienten mit axialer SpA in allen Sektoren beteiligt sind (primäre, sekundäre und tertiäre Versorgungsebene, ambulant als auch stationär unter Berücksichtigung der Rehabilitation). Die Leitlinie bezieht sich somit sowohl auf die primärärztliche Versorgung als auch auf die fachärztliche Versorgung. Die inter- bzw. multidisziplinäre Strategie/Bewertung der Leitlinie wird ausdrücklich betont, dies ist ja Voraussetzung für eine S3-Leitlinie. Die Patientenzielgruppe umfasst alle erwachsenen Patienten mit einer axialen SpA. Die Leitlinie richtet sich darüber hinaus auch an Angehörige von Patienten mit axialer SpA. Betroffene und deren Angehörige werden durch eine speziell für sie erstellte Patientenleitlinie angesprochen.

\section{Präambel}

Aufgrund der häufig anzutreffenden Unsicherheit, Erkrankungen aus dem Formenkreis der SpA korrekt zu diagnostizieren und eine adäquate Therapie einzuleiten, hat sich die Konsensusgruppe entschlossen, eine Präambel zu formulieren. Diese Statements überlappen sich zum Teil mit denjenigen Empfehlungen, die in 
der internationalen ASAS(Assessment of Spondyloarthritis international Society)/ EULAR(European League Against Rheumatism)-Empfehlung zum Management der axialen SpA getroffen wurden [21]. Aufgrund des vielschichtigen klinischen Bildes sei hier insbesondere auf die Notwendigkeit eines koordinierten multidisziplinären Vorgehens hingewiesen. Nationale Stellungnahmen und Empfehlungen zu übergeordneten Themenkomplexen wie Impfempfehlungen und Familienplanung behalten ihre Gültigkeit.

\section{Empfehlung/Statement}

\section{2-1}

Unter einer axialen Spondyloarthritis (SpA) versteht man eine entzündliche Wirbelsäulenerkrankung aus dem rheumatischen Formenkreis, die mit verschiedenen muskuloskelettalen und extraskelettalen Manifestationen vergesellschaftet sein kann.

Empfehlungsgrad: Statement

Evidenz: -

\section{2-2}

Die axiale Spondyloarthritis ( $\mathrm{SpA})$ ist eine potenziell schwerwiegende Erkrankung mit unterschiedlichen Krankheitserscheinungen und -verläufen, welche vor allem auch unter Berücksichtigung von extraartikulären Manifestationen wie Psoriasis, Uveitis und chronischentzündlichen Darmerkrankungen und Komorbiditäten (z. B. kardiovaskuläre Erkrankungen) ein koordiniertes multidisziplinäres Vorgehen erfordert. Empfehlungsgrad: Statement Evidenz: -

\section{2-3}

Die Koordination der Versorgung sowie die Zuständigkeit für Komorbiditäten und deren Risikofaktoren soll zwischen dem Rheumatologen und dem Hausarzt abgesprochen werden. Empfehlungsgrad: Evidenz: Expertenkonsens
2-4

Patienten, die immunsuppressiv behandelt werden, sollen gemäß den STIKOEmpfehlungen geimpft werden. Empfehlungsgrad: -

Evidenz: Expertenkonsens

\section{2-5}

Das primäre Ziel in der Behandlung von Patienten mit axialer SpA ist die Optimierung der Lebensqualität durch das Erreichen einer weitgehenden Symptomfreiheit, die Reduktion der Entzündung, Verhinderung von strukturellen Schäden und die Aufrechterhaltung bzw. Normalisierung von Funktion, Aktivität und sozialer Partizipation einschließlich der Arbeits- und Erwerbsfähigkeit.

Empfehlungsgrad: -

Evidenz: Expertenkonsens

\section{2-6}

Die Behandlung der Erkrankung sollte auf die beste Betreuung ausgerichtet sein und auf der Grundlage einer partizipativen Entscheidungsfindung zwischen Patient und behandelndem Arzt erfolgen.

Empfehlungsgrad:-

Evidenz: Expertenkonsens

\section{Klinische Symptomatik}

\section{Schlüsselfrage 7}

- Welche muskuloskeletalen Symptome können bei Patienten mit SpA auftreten, und wie sollte das Assessment aussehen (Häufigkeit und welche)?

\subsection{Muskuloskeletales System}

\subsubsection{Symptome}

\subsubsection{Chronischer Rückenschmerz.}

Das führende Hauptsymptom bei Patienten mit einer axialen SpA sind chronische Rückenschmerzen, d.h. dass die Rückenschmerzen länger als 12 Wochen bestehen. Prinzipiell kann die gesamte Wirbelsäule schmerzhaft betroffen sein, vorzugsweise sind aber sakroiliakale vor lumbalen und untere thorakale vor zervikalen und oberen thorakalen Strukturen betroffen. Da ca. 75\% der Patienten mit axialer SpA unter einem entzündlichen Rückenschmerz (ERS) leiden, kommt dieser Form des chronischen Rückenschmerzes eine besondere Bedeutung zu [1, 22]. Populationsbasierte Daten aus Großbritannien legen nahe, dass die Prävalenz des ERS in der Primärversorgung zwischen 1,7 und 3,4\% schwankt [23].

Als spezifisches Zeichen einer SpA finden die Symptome des ERS zum ersten Mal 1977 Eingang in die von Calin entwickelten Screening-Kriterien für AS [24]. Die Beschreibungen der einzelnen Charakteristika des ERS sind im Folgenden modifiziert worden $[25,26]$. Es gibt keine Einigung international unter den Experten, welche Modifikation zu bevorzugen ist. Eine neuere Untersuchung zeigt, dass die Calin-Kriterien eine hohe Sensitivität, die Berlin-Kriterien eine hohe Spezifität und die ASAS-Kriterien eine gleich hohe Sensitivität und Spezifität aufweisen [27]. In einer Untersuchung anhand Daten der DESIR-Kohorte zeigte sich aber auch, dass eine niedrige Übereinstimmung der Kriteriensets mit der Meinung des Arztes vorliegt [28]. Der charakteristische ERS zeichnet sich dadurch aus, dass er bei jungen Patienten auftritt, die Schmerzphasen schleichend beginnen, die Schmerzen oft in der Nacht auftreten und dass die Schmerzen sich durch Bewegung, nicht aber durch Ruhe bessern lassen (•Tab. 1). Keines der Charakteristika des ERS eignet sich als einzelne Variable zur Differenzierung zwischen Patienten mit axialer SpA und nichtspezifischem Rückenschmerz. Die Frage nach „Morgensteifigkeit“ und nach dem „nächtlichen Aufwachen in der 2. Nachthälfte" scheint in einer Studie am besten zur Differenzierung geeignet $\mathrm{zu}$ sein, in einer anderen Studie war dies die Frage nach „Besserung durch Bewegung“ sowie „Gesäßschmerzen“ $[25,27]$.

Neben dem Rückenschmerz ist die zunehmende Steifheit der Wirbelsäule für die Patienten mit axialer SpA von zentraler Bedeutung. Der untersuchende Arzt sollte versuchen $\mathrm{zu}$ differenzieren, ob die Einschränkung der Wirbelsäulenbeweglichkeit auf strukturelle Schäden oder auf eine entzündliche Komponente zurückzuführen ist [10, 29], denn 
Tab. 1 Variablen der verschiedenen Definitionen des entzündlichen Rückenschmerzes

\begin{tabular}{|c|c|c|c|}
\hline & $\begin{array}{l}\text { Calin: Historische } \\
\text { Definition [24] }\end{array}$ & $\begin{array}{l}\text { Rudwaleit: Basierend } \\
\text { auf Studiendaten [25] }\end{array}$ & $\begin{array}{l}\text { Sieper: Basierend auf } \\
\text { Expertenkonsensus [26] }\end{array}$ \\
\hline Alter & $<40$ Jahre & $<45$ Jahre & $\begin{array}{l}\leq 40 \text { Jahre (Odds Ratio } \\
{[\text { OR]: } 9,9)}\end{array}$ \\
\hline Dauer & $\geq 3$ Monate & $\geq 3$ Monate & - \\
\hline Beginn & Schleichender Beginn & - & $\begin{array}{l}\text { Schleichender Beginn } \\
\text { (OR: 12,7) }\end{array}$ \\
\hline \multirow[t]{5}{*}{ Klinik } & Morgensteifigkeit & Morgensteifigkeit $>30 \mathrm{~min}$ & - \\
\hline & $\begin{array}{l}\text { Besserung durch } \\
\text { Bewegung }\end{array}$ & - & $\begin{array}{l}\text { Besserung durch Bewegung } \\
\text { (OR: } 23,1)\end{array}$ \\
\hline & - & $\begin{array}{l}\text { Keine Verbesserung durch } \\
\text { Ruhe }\end{array}$ & $\begin{array}{l}\text { Keine Verbesserung durch } \\
\text { Ruhe }(\mathrm{OR} 7,7)\end{array}$ \\
\hline & - & $\begin{array}{l}\text { Alternierender Gesäß- } \\
\text { schmerz }\end{array}$ & - \\
\hline & - & $\begin{array}{l}\text { Aufwachen in der } \\
\text { 2. Nachthälfte }\end{array}$ & $\begin{array}{l}\text { Nächtliche Schmerzen } \\
\text { (OR: 20,4) }\end{array}$ \\
\hline Sensitivität ${ }^{\mathrm{a}}$ & $\begin{array}{l}\text { Bei } 4 \text { von } 5 \text { Kriterien: } \\
90 \%\end{array}$ & Bei 2 von 4 Kriterien: $70 \%$ & Bei 4 von 5 Kriterien: $80 \%$ \\
\hline Spezifität ${ }^{b}$ & $\begin{array}{l}\text { Bei } 4 \text { von } 5 \text { Kriterien: } \\
52 \%\end{array}$ & Bei 2 von 4 Kriterien: $81 \%$ & Bei 4 von 5 Kriterien: $72 \%$ \\
\hline
\end{tabular}

dies hat mögliche Auswirkungen auf die Therapieentscheidung, da bei eingeschränkter Wirbelsäulenbeweglichkeit aufgrund von Entzündung eine antiinflammatorische Therapie $\mathrm{zu}$ einer Verbesserung der Wirbelsäulenbeweglichkeit führen kann - im Gegensatz dazu ist dieses bei einer eingeschränkten Wirbelsäulenbeweglichkeit durch strukturelle Schäden nicht zu erwarten. Mit zunehmenden strukturellen Schäden im Bereich der Wirbelsäule kann es zu einer veränderten Körperhaltung und Statik kommen: Vertikalstellung des Beckens, Ausgleich der Lendenlordose, Verstärkung der Brustkyphose und der Halswirbelsäulen(HWS)-Lordose, Tendenz der Beugestellung der Hüft- und Kniegelenke, Lateraldrehen der Schulterblätter, Überdehnung der Bauchmuskulatur mit dominierender Bauchatmung, Atrophie und Überdehnung der Lumbalmuskulatur, Hypotonie der Gesäßund Nackenmuskulatur und Mangel an Mitbewegungen der Wirbelsäule beim Gehen.

Als weitere wirbelsäulennahe Gelenke können auch die Gelenke im Bereich des Sternums entzündliche Veränderungen aufweisen. Hier ist häufig eine Schnitt- bilddiagnostik zur korrekten Diagnosestellung hilfreich [30].

\subsubsection{Beteiligung peripherer Gelen-}

ke. Patienten mit axialer SpA können zusätzlich zu der axialen Beteiligung an einer peripheren Arthritis leiden, die sich häufig als asymmetrische Oligoarthritis ( ${ }^{\star}$ M14.8) bevorzugt der unteren Extremität meist unter Aussparung kleinerer Gelenke darstellt [1]. Zirka 30\% der Patienten leiden an einer Arthritis oder Enthesitis (Sehnenansatzentzündung) (s. Abschn. 3.1.1.3). Eine Daktylitis tritt seltener als $30 \%$ auf. Mit einer Daktylitis wird die Entzündung eines ganzen Fingers oder Zehs beschrieben, d.h. es sind alle Gelenke mit umgebender Beteiligung der Weichteile im Strahl entzündet und nicht nur einzelne Gelenke. In einer französischen Kohorte wiesen 21,5\% der Patienten mit SpA eine Daktylitis auf (Gruppe bestand aus 190 [69,1\%] Patienten mit axSpA, 49 [17,8\%] PsA, 37 [13,4\%] uSpA, 23 [8,4\%] SpA-asso5 [1,8\%] reaktive Arthritis) [31]. Die Daktylitis manifestiert sich häufiger an der unteren $(78,0 \%)$ als an der oberen Extremität $(42,4 \%)$. Die Daktylitis weist eine hohe diagnostische Aussagekraft ziierte CED, 9 [3,3\%] juvenile SpA und bei Patienten mit chronischen Rückenschmerzen auf (pos. Likelihood-Ratio [LR] 4,1) (s. Abschn. 5.2.4) [32].

Die periphere Arthritis zeigt im Vergleich zur rheumatoiden Arthritis (RA) weniger häufig einen destruierenden Verlauf [1]. Der Befall der Hüftgelenke ist prognostisch ungünstig (s. Abschn. 7.2). Patienten mit peripherer Beteiligung weisen eine Einschränkung der körperlichen Funktionsfähigkeit auf, die sich auch in dem auf die Achsenskelettmanifestation fokussierten Fragebogen Bath Ankylosing Spondylitis Functioning Index (BASFI) niederschlägt [33].

3.1.1.3 Enthesitis. Die Entzündung an Sehnenansätzen ist pathognomonisch für die Patienten mit axialer SpA, wobei auch wieder die untere Extremität am häufigsten betroffen ist. Patienten mit AS wiesen im Vergleich mit gesunden Kontrollen sonographisch doppelt so viel Enthesophyten wie Kontrollpatienten auf [34]. In einer weiteren kontrollierten Studie zeigte sich in der klinischen Untersuchung bei 9,3\% der Patienten eine Enthesiopathie, wohingegen sich bei $60 \%$ der klinisch unauffälligen Enthesen sonographisch mindestens ein Ultraschallzeichen positiv darstellte [35]. In der Regel wird die Enthesitis klinisch diagnostiziert, indem die Druckschmerzhaftigkeit eines anatomischen Areals überprüft wird, das häufig von einer Enthesitis betroffen ist. Die in klinischen Studien am häufigsten verwendeten Instrumente überprüfen den Druckschmerz in folgenden Bereichen: kostochondral, Spina iliaca anterior superior, Processus spinosus, Achillessehne [36, 37]. Patienten mit Enthesitis haben allgemein eine höhere Krankheitsaktivität (gemessen mit dem Bath Ankylosing Spondylitis Disease Activity Index [BASDAI]) und eine stärkere Einschränkung der Funktionsfähigkeit (gemessen mit dem BASFI) [36].

Die Angaben zu den diagnostischen Testeigenschaften von Anamnese, körperlicher Untersuchung, Labor und Medikation finden sich in - Tab. 2.

Kohortendaten zeigen, dass Frauen im Vergleich zu Männern häufiger höhere Schmerzscores, eine stärkere FatigueSymptomatik, eine höhere Krankheits- 
Tab. 2 Diagnostische Testeigenschaften von Anamnese, körperlicher Untersuchung, Labor und Medikation. (Adaptiert nach $[42,43]$ )

\begin{tabular}{|c|c|c|c|c|c|}
\hline Parameter & $\begin{array}{l}\text { Sensi- } \\
\text { tivität } \\
(\%)\end{array}$ & $\begin{array}{l}\text { Spezifität (\%) } \\
\text { (AS vs. MRS- } \\
\text { Kontrollen) }\end{array}$ & $\begin{array}{l}\text { Likelihood- } \\
\text { Ratio (LR) }\end{array}$ & $\begin{array}{l}\text { Spezifität } \\
\text { (AS vs. alle } \\
\text { Kontrollen) }\end{array}$ & $\begin{array}{l}\text { Likelihood- } \\
\text { Ratio (LR) }^{\text {a }}\end{array}$ \\
\hline $\begin{array}{l}\text { Entzündlicher } \\
\text { Rücken- } \\
\text { schmerz }\end{array}$ & 77,3 & 81,6 & 4,5 & 89,7 & 7,5 \\
\hline $\begin{array}{l}\text { Alternierender } \\
\text { Gesäßschmerz }\end{array}$ & 39,7 & 85,8 & 2,8 & 96,3 & 10,9 \\
\hline $\begin{array}{l}\text { Beginn vor } \\
\text { dem } 45 . \text { Le- } \\
\text { bensjahr }\end{array}$ & 93,7 & 14,0 & 1,1 & 14 & 1,1 \\
\hline $\begin{array}{l}\text { Periphere } \\
\text { Arthritis }\end{array}$ & 45,5 & 96,1 & 11,7 & 72,8 & 1,7 \\
\hline Daktylitis & 3,5 & $x$ & $x$ & 96,8 & 1,1 \\
\hline Uveitis & 24,7 & 100 & $\infty$ & 99 & 24,6 \\
\hline Enthesitis & 31,8 & 92,2 & 4,1 & 92,5 & 4,2 \\
\hline $\begin{array}{l}\text { Positive Famili- } \\
\text { enanamnese }\end{array}$ & 22,3 & 100 & $\infty$ & 96,4 & 6,2 \\
\hline $\begin{array}{l}\text { Gutes Anspre- } \\
\text { chen auf NSAR }\end{array}$ & 57,9 & 86 & 4,1 & 76,3 & 2,4 \\
\hline $\begin{array}{l}\text { HLA-B27 posi- } \\
\text { tiv }\end{array}$ & 88,0 & 92,6 & 11,9 & 93,2 & 12,6 \\
\hline \multicolumn{6}{|c|}{$\begin{array}{l}\text { AS ankylosierende Spondylitis, MRS mechanischer Rückenschmerz, NSAR nichtsteroidale Antirheu- } \\
\text { matika, } x \text { keine Daten vorhanden } \\
\left.{ }^{a} \mathrm{LR}=\text { Sensitivität/(Spezifität }-1\right)\end{array}$} \\
\hline
\end{tabular}

aktivität (BASDAI) sowie eine stärkere Beeinträchtigung der körperlichen Funktionsfähigkeit bei allerdings gleichzeitig geringeren strukturellen Veränderungen (Röntgen) angeben [38-40]. In der körperlichen Untersuchung wird bei Frauen häufiger eine Enthesitis bzw. Daktylitis als bei Männern diagnostiziert [41]. Männliche axSpA-Patienten weisen dagegen einen schweren Strukturschaden an der Wirbelsäule auf (s. Abschn. 7.2) [39].

3.1.1.4 Veränderung der Knochendichte und Frakturrisiko. Neben genannten primär entzündlichen muskuloskeletalen Manifestationen findet sich bei SpA-Patienten häufig eine verminderte Knochendichte. Die Mehrzahl der Patienten mit AS zeigt eine Beeinträchtigung der Knochendichte (Osteopenie 59\%, Osteoporose 18\%) [44, 45]. Aber auch schon Patienten mit einer kurzen Erkrankungsdauer weisen eine erniedrigte Knochendichte auf [46]; $14,7 \%$ der 265 Patienten aus der französischen DESIR-Kohorte mit axSpAPatienten und kurzer Erkrankungsdauer wiesen eine Knochendichte mit einem Z-Score $\leq 2$ (mindestens einseitig) auf. Folgende Faktoren sind bei AS-Patienten mit einem erhöhten Risiko für eine erniedrigte Knochendichte assoziiert: männliches Geschlecht (OR 3,87 [95\%CI 1,21-7,36]), hohe Krankheitsaktivität (OR [ASDAS] 2,83 [95\%-CI 1,36-4,76] bzw. OR [MRT Sakroiliitis] 2,83 [95\%CI 1,77-6,23]) sowie eine röntgenologische Sakroiliitis (OR 2,93 [95\%-CI 1,82-4,45]) [45, 47]. Patienten mit AS haben daher ein hohes Frakturrisiko, insbesondere der Wirbelsäule (Frakturen allgemein OR 1,54 [95\%-CI 1,26-1,89]), Frakturen der Wirbelsäule 5,42 (95\%CI 2,50-11,70) und Frakturen anderer Gelenke 1,39 (95\%-CI 1,12-1,73) [48]. Eine erniedrigte Knochenmineraldichte im Bereich des Femurs korreliert mit dem Risiko, eine vertebrale Fraktur zu erleiden [49]. Patienten unter einerTNFi-Therapie zeigen einen signifikanten Anstieg der Knochendichte an der Wirbelsäule, nicht jedoch an der Hüfte $[46,50,51]$ Dies legt nahe, dass die erniedrigte Knochendichte zumindest teilweise entzündlich bedingt ist.
Gemäß einer Registerstudie sind diese Frakturen $\mathrm{zu} 82 \%$ in der Brustwirbelsäule lokalisiert [52]. Nach adäquatem Trauma (z. B. Sturz, Verkehrsunfall) sind bei AS-Patienten in erster Linie die untere HWS, gefolgt von der unteren Brustwirbelsäule frakturiert (s. Abschn. 8.6.3) [53]. Ursache für diese große Diskrepanz sind die sehr unterschiedlichen Erfassungsmethoden und Verletzungsgrade in der zu bewertenden Literatur. Osteoporosebedingte spontane Keilwirbelbildungen durch Impressionen der Deckplatte bei intakt gebliebener dorsaler Säule können nicht gleichgestellt werden mit der hochgradigen Instabilität einer durch adäquates Trauma hervorgerufenen Fraktur aller knöchernen Anteile der Wirbelsäule. Bei einem ASPatienten frakturieren diese wie ein langer Röhrenknochen, meist in Form einer distrahierenden Verletzung. Daher sind Letztere hoch instabil und mit einem hohen initialen und Spät-Lähmungsrisiko verbunden (persönliche Kommunikation der beteiligten Wirbelsäulenchirurgen). AS-Patienten mit einer akuten Wirbelfraktur sollten in einem spezialisierten operativen Wirbelsäulenzentrum vorgestellt werden (s. Abschn. 8.6.3). Die in diesem Kapitel zusammengefassten Ergebnisse zur Veränderung der Knochendichte bei AS-Patienten sind vor allem vor dem Hintergrund interessant, dass zumindest AS-Patienten ein erhöhtes Sturzrisiko aufweisen. In einer türkischen Kohorte berichteten 13,1\% der Patienten über einen Sturz, der innerhalb der letzten 12 Monate aufgetreten war [54]. Patienten mit Stürzen haben ein höheres Durchschnittsalter, eine längere Krankheitsdauer und eine stärker eingeschränkte Funktionsfähigkeit.

Das Risiko einer peripheren Fraktur wie Radiusfraktur oder einer Oberschenkelhalsfraktur war bei AS-Patienten gemäß einer umfangreichen Fall-KontrollStudie nicht signifikant erhöht (OR 1,21 [95\%-CI 0,87-1,69] bzw. OR 0,77 [95\%CI 0,43-1,37]) [50]. Das Risiko einer Fraktur war bei Patienten, die mit nichtsteroidalen Antirheumatika (NSAR) therapiert wurden, niedriger (OR 0,65 [95\%CI 0,50-0,84]) [50]. Alter, hoher BASFI und BASRI (Ausmaß struktureller Läsio- 
nen in der Wirbelsäule) sind häufiger mit Frakturen assoziiert [52].

\subsubsection{Untersuchung}

3.1.2.1 Körperliche Untersuchung. Bei der körperlichen Untersuchung eines $\mathrm{Pa}$ tienten mit SpA sollte das Augenmerk des Untersuchers besonders auf der Erfassung der Wirbelsäulenbeweglichkeit, von Haltungsveränderungen, der Beteiligung peripherer Strukturen und extraskeletaler Manifestationen (Abschn. 3.2) liegen. $\mathrm{Zu}$ beachten ist, dass alle unten angegebenen Maße altersabhängige Normwerte haben und dass alle unten angegebenen Indizes lediglich bei Patienten mit AS untersucht wurden.

\subsection{Wirbelsäulenbeweglichkeit.}

Die Überprüfung der Wirbelsäulenbeweglichkeit soll alle Wirbelsäulenabschnitte und alle Bewegungsrichtungen der Wirbelsäule einbeziehen. Wie oben bereits erwähnt, kann die Einschränkung der Wirbelsäulenbeweglichkeit sowohl durch entzündliche Prozesse als auch durch strukturelle Veränderungen verursacht werden. Auch der Einfluss von Veränderungen im SIG auf die Wirbelsäulenbeweglichkeit sollte berücksichtigt werden [55]. Es gibt eine Vielzahl von Untersuchungstechniken zur Erfassung der Wirbelsäulenbeweglichkeit. In einem Review sind die verschiedenen Untersuchungstechniken hinsichtlich ihrer Validität zusammengefasst worden [56].

Bei der Lendenwirbelsäule sollen die Anteflexion und die Lateralflexion untersucht werden. Dies erfolgt mittels modifizierter Untersuchung nach Schober. Dabei wird ein definierter Abschnitt der unteren Lendenwirbelsäule (LWS) (Dornfortsatz 5. Lendenwirbel und $10 \mathrm{~cm}$ nach kranial) in Normalhaltung und nach Anteflexion vermessen (Norm $>4 \mathrm{~cm}$ ). Das Schober-Maß ist für kurzfristige Verlaufsuntersuchungen weniger sensitiv als die Messung der Lateralbeweglichkeit der Wirbelsäule $($ Norm $>10 \mathrm{~cm})$, die Zuverlässigkeit (Reliabilität) ist jedoch gut (IntraclassKorrelation >0,9) [56, 57]. Die zervikale Rotation wird am sitzenden Patienten gemessen (Norm $>70^{\circ}$ ). Zur Beurteilung der Kyphosierung können die Messung des Tragus-Wand-Abstandes als auch die Messung des Hinterhaupt-WandAbstandes (HWA) durchgeführt werden [58]. Die Messung des HWA ist als zuverlässige Methode in einer longitudinalen Studie über 2 Jahre validiert worden (Intraclass-Korrelation >0,9) [59]. Die Messung der Thoraxexkursion erfolgt vor und nach maximaler In- bzw. Exspiration im 4. ICR mithilfe eines flexiblen Maßbandes (Norm $>5 \mathrm{~cm}$ ). Die Untersuchung der Thoraxexkursion gibt Rückschlüsse auf den Befall bzw. die noch vorhandene Beweglichkeit der kostosternalen und kostovertebralen Gelenke, die Zuverlässigkeit (Reliabilität) ist jedoch schlecht [60, 61]. Querschnittstudien haben eine gute Korrelation zwischen Messungen der Wirbelsäulenbeweglichkeit und radiologischen Veränderungen bei Patienten mit AS gezeigt [57, 62, 63]. In Fall-Kontroll-Studien konnte die Diskriminationsfähigkeit der Wirbelsäulenvermessung zwischen gesunden Kontrollen und AS-Patienten gezeigt werden [64].

Die Auswirkungen einer eingeschränkten körperlichen Funktionsfähigkeit können durch den Fragebogen BASFI erfasst werden (s. Abschn. 3.1.2.2). Die diagnostischen Testeigenschaften der körperlichen Untersuchung weisen eine breite Spannweite auf [42]. Das Summenmaß für die Beweglichkeitseinschränkung der Wirbelsäule kann mithilfe des BASMI (Bath Ankylosing Spondylitis Metrology Index) erfasst werden [65]. In diesem Score ist die Beurteilung der lumbalen Flexion, der lateralen lumbalen Flexion, des Tragus-Wand-Abstandes, des maximalen Intermalleolarabstandes (indirekte Messung der Hüftgelenkbeweglichkeit s. Abschn. 3.1.2.1.2) und des HWS-Rotationswinkels enthalten.

3.1.2.1.2 Periphere Manifestation. Im Fokus der Untersuchung sollte die untere Extremität stehen, und es sollten die Anzahl der druckschmerzhaften und die Anzahl der geschwollenen Gelenke dokumentiert werden. Im Vergleich zur RA und zum DAS-28 gibt es für die axiale SpA kein validiertes Instrument zur Erfassung der peripheren Gelenkmanifestation. Zur Erfassung des Bewegungsausmaßes wird im klinischen Alltag die Neutral-Null-Methode verwendet.
Zur Dokumentation der Hüftgelenkbeweglichkeit eignet sich die Messung des maximalen Intermalleolarabstandes am stehenden oder liegenden Patienten (Norm $>100 \mathrm{~cm}$ ). Neben der peripheren Arthritis sollte die Aufmerksamkeit des Untersuchers auch auf die Erfassung von möglichen Enthesitiden gerichtet sein.

3.1.2.2 Assessment. Verschiedene Assessments werden bei Patienten mit einer axialen SpA sowohl in der klinischen Routine als auch in Studien eingesetzt.

3.1.2.2.1 Bath-Indizes. Die Bath-Indizes sind Kompositionsmessinstrumente, die für Patienten mit AS entwickelt worden sind, um die Krankheitsaktivität (BASDAI) (s. Abschn. 7.1) und die körperliche Funktionsfähigkeit (BASFI) zu erfassen $[66,67]$. Der BASMI fasst die Ergebnisse der körperlichen Untersuchung zusammen, wird aber überwiegend nur in Studien erhoben [65].

Die Einschränkung der körperlichen Funktionsfähigkeit kann mit der in Deutschland validierten Version des BASFI (Bath Ankylosing Spondylitis Functioning Index) erhoben werden $[66,68]$. In diesem Fragebogen werden 10 Alltagsverrichtungen aufgeführt, die anhand einer numerischen Ratingskala von 0-10 eingeschätzt werden müssen. Die Summe des BASFI liegt zwischen 0 (keine Einschränkung) und 10 (sehr starke Einschränkung). Die mit dem BASFI dokumentierte Einschränkung der körperlichen Funktionsfähigkeit ist Folge einer erhöhten Entzündungsaktivität, bereits bestehender röntgenologischer Veränderungen und/oder einer Hüftgelenkbeteiligung [69]. Zusätzlich zu den strukturellen Veränderungen sind eine längere Krankheitsdauer, eine größere Anzahl an Komorbiditäten und eine körperlich anstrengende Berufstätigkeit mit einem höheren BASFI-Wert assoziiert [70]. Neben dem BASFI kann die Einschränkung der körperlichen Funktionsfähigkeit auch mit dem Health Assessment Questionnaire (HAQ) in der Modifikation für Spondyloarthritiden (HAQ-S) beurteilt werden [71]. Der BASFI hat sich aufgrund der einfacheren Berechnung in der klinischen Anwendung durchgesetzt. 
Tab. 3 Prävalenz der extraartikulären Manifestationen und Komorbiditäten

\begin{tabular}{|l|c|c|}
\hline $\begin{array}{l}\text { Extraartikuläre } \\
\text { Manifestation }\end{array}$ & $\begin{array}{c}\text { Prävalenz bei } \\
\text { AS-Patienten (\%) [72] }\end{array}$ & $\begin{array}{l}\text { Standardisierte Morbiditätsrate, } \\
\text { SMR (95\%-CI) [78] }\end{array}$ \\
\hline Uveitis & $30-50$ & $34,35(28,55-40,98)$ \\
\hline CED & $4-10$ & $9,28(7,07-11,97)$ \\
\hline Psoriasis & $10-25$ & $2,94(2,06-4,08)$ \\
\hline Aorteninsuffizienz & $1-10$ & - \\
\hline $\begin{array}{l}\text { Reizleitungsstörung des } \\
\text { Herzens }\end{array}$ & $1-33$ & $3,97(1,90-7,30)$ \\
\hline Koronare Herzerkrankung & - & $2,20(1,77-2,7)$ \\
\hline Arterielle Hypertonie & - & $1,98(1,72-2,28)$ \\
\hline Diabetes mellitus & - & $1,41(1,10-1,78)$ \\
\hline Lungenbeteiligung & $9-88$ & - \\
\hline Osteopenie & $35-59$ & - \\
\hline Osteoporose & $11-18$ & $4,33(2,96-6,11)$ \\
\hline
\end{tabular}

\subsection{Weitere Patient-reported Out-}

comes. Schmerz: Für die Erfassung von Schmerz wird eine visuelle Analogskala (VAS 0-10 oder 0-100) oder eine numerische Ratingskala (NRS 0-10) verwendet. Hierbei kann nach Schmerzen im Allgemeinen als auch nach nächtlichen Wirbelsäulenschmerzen getrennt untersucht werden.

Steifheit: Patienten mit axialer SpA berichten sehr häufig über eine Steifheit der Wirbelsäule. Es existiert kein validiertes Messinstrument. Alternativ können die Fragen 5 und 6 des BASDAI herangezogen werden, die sich auf Ausprägung und Länge der Steifheit beziehen.

\section{Empfehlung/Statement}

\section{3-1}

Bei Patienten mit chronischen Rückenschmerzen ( $\geq 12$ Wochen) sollten die Charakteristika des entzündlichen Rückenschmerzes erfragt werden (Morgensteifigkeit $>30 \mathrm{~min}$, Aufwachen in der 2. Nachthälfte, Besserung durch Bewegung, keine Verbesserung durch Ruhe, schleichender Beginn, Alter bei Beginn $\leq 45$ Jahre). Hierbei ist zu beachten, dass nur zirka $75 \%$ der Patienten mit SpA diese typischen Charakteristika aufweisen.

Empfehlungsgrad: $B$

Evidenz: 3b
3-2

Bei einer raschen Verschlimmerung oder Veränderung der Schmerzsymptomatik der Wirbelsäule sollte neben einer Entzündung auch eine Fraktur (auch nach geringfügigem Trauma) als mögliche Ursache in Betracht gezogen werden. Eine entsprechende Diagnostik inklusive Bildgebung (Rö/CT/MRT) sollte zeitnah veranlasst werden. Bei Wirbelsäulenverletzungen (Frakturen) sollte aufgrund des höheren Instabilitätspotenzials nur in Ausnahmefällen eine konservative der operativen Therapie vorgezogen werden.

Empfehlungsgrad: $B$

Evidenz: 4

\section{Kommentar:}

Die Empfehlung wurde von einer " 0 "auf eine "B"-Empfehlung hochgestuft, weil es aufgrund ethischer Verpflichtung keine prospektiven Daten geben kann.

\section{3-3}

Bei Patienten mit axialer Spondyloarthritis soll regelmäßig und in Abhängigkeit vom Krankheitsverlauf geprüft werden, ob sich die Wirbelsäulenbeweglichkeit verschlechtert. Empfehlungsgrad:

Evidenz: Expertenkonsens
3-4

In der Betreuung von Patienten mit axialer Spondyloarthritis sollen regelmäßig und in Abhängigkeit des Krankheitsverlaufes die Krankheitsaktivität und körperliche Funktionsfähigkeit ggf. auch unter Zuhilfenahme von Fragebögen (BASDAI und BASFI) bzw. Composite Scores (ASDAS) erfasst werden. Empfehlungsgrad: Evidenz: Expertenkonsens

\subsection{Extraskeletale Manifestation}

\section{Schlüsselfrage 6}

- Welche extraskeletalen Manifestationen können bei Patienten mit SpA auftreten, und wie sollte das Assessment aussehen (Häufigkeit und welche)?

\section{Schlüsselfrage 22}

- Wann sollte eine Mitbehandlung durch den Spezialisten erfolgen?

Extraartikuläre Manifestationen (EAM) an den Augen, der Haut und am Darm liegen bei bis $\mathrm{zu} 40 \%$ der Patienten vor (• Tab. 3; [72, 73]). Die meisten Studien liegen zu Patienten mit AS vor. Einige Kohortenstudien berichten jedoch auch von Häufigkeiten der EAM bei Patienten mit nr-axSpA [74, 75]. Am häufigsten leiden Patienten mit einer axSpA an einer Uveitis $(25,8 \%)$, einer Psoriasis (9,3\%) oder einer chronisch entzündlichen Darmerkrankung (CED) (6,8\%) [76]. Bei bis zu $10 \%$ der Patienten liegt eine Kombination von EAM vor [72]. Registerdaten aus Spanien haben ähnlich hohe Prävalenzen bei Patienten mit SpA gezeigt [77]. Die Prävalenz der EAM steigt mit zunehmender Krankheitsdauer an [76]. Schwedische Registerdaten zeigen eine erhöhte standardisierte Morbiditätsrate (SMR) bei AS-Patienten mit Uveitis (SMR 34,35 [95\%-CI 28,55-40,98]) und CED (SMR 9,28 [95\%-CI 7,07-11,97]) (• Tab. 3; [78]). Patienten mit kardiovaskulären Erkrankungen und Osteoporose weisen eine geringere Erhöhung der SMR auf (• Tab. 3).

Das zugrundeliegende pathophysiologische Konzept der extraskeletalen Manifestationen ist nicht vollständig geklärt. Bei der anterioren Uveitis liegt eine As- 
soziation mit $H L A-B 27$ vor, dies ist bei Patienten mit extraskeletalen Manifestationen an Haut oder Darm nicht der Fall.

Die Beteiligung des Knochens mit einer verminderten Knochendichte nimmt insofern eine Sonderstellung ein, als hier mehrere Faktoren für die Ausbildung und Ausprägung der Osteoporose verantwortlich $\mathrm{zu}$ sein scheinen, unter anderem chronische Entzündung und relative Immobilität.

Das Vorhandensein von extraskeletalen Manifestationen hat einen wesentlichen Einfluss auf die Lebensqualität der Patienten. So konnte z.B. gezeigt werden, dass insbesondere die psychische Gesundheit der Patienten beeinträchtigt ist [79]. Klinische Symptome wie Diarrhö, Haut- und Nagelprobleme, Augenschmerzen- und/oder -rötungen sowie unerklärter Gewichtsverlust sollten Anlass zu weiteren, in der Regel fachspezifischen Untersuchungen geben.

\subsubsection{Augenbeteiligung}

Bei Patienten mit SpA manifestiert sich die Augenbeteiligung in der Regel als akute anteriore Uveitis (AAU) und kommt bei 18-40\% der AS-Patienten vor $[73,80,81]$. In einer Metaanalyse mit 29.877 Patienten wurde die Prävalenz mit $32,7 \%$ angegeben, bei AS-Patienten ist sie am höchsten (33,2\%) [82]. In dieser Analyse war die Prävalenz bei HLAB27-positiven Patienten am höchsten (OR 4,2) [82]. In der niederländischen OASIS-Kohorte war das Vorhandensein einer AAU mit erhöhtem Alter (OR 1,04 [95\%-CI 1,01-1,07]), einer längeren Symptomdauer (OR 1,05 [95\%-CI $1,02-1,08])$ und mit vermehrtem röntgenologischem Schaden (OR 1,02 [95\%CI 1,00-1,04]) assoziiert [83]. In einer französischen Kohorte traten bei 11,7\% der Patienten Komplikationen auf [81]. Weitere genetische Assoziationen mit dem Auftreten einer AAU sind beschrieben worden [84]. Umgekehrt weisen Patienten mit einer HLA-B27-assoziierten Uveitis ohne (bisher) bekannte SpA eine hohe Wahrscheinlichkeit auf, eine Sakroiliitis oder periphere Arthritis zu entwickeln [85]. Die AAU ist durch ein schmerzhaftes rotes Auge charakterisiert und tritt meistens akut und typischerweise unilateral auf [86]. Be- troffene Patienten berichten von einer Photophobie, verschwommenem Sehen und einer überschießenden Tränenproduktion. Die AAU bildet sich in der Regel nach 1 bis 3 Monaten zurück mit einer deutlichen Tendenz zum Rezidiv in den nächsten Monaten. Patienten mit einer Augenbeteiligung sollten ophthalmologisch mitbetreut werden. Durch eine effektive antiinflammatorische Therapie und „Ruhigstellung“" der Pupille durch Mydriatika sollen Komplikationen und Risiken für eine dauerhafte Seheinschränkung durch Hypopyon, Synechien, Katarakt, Glaukom oder Makulaödem verringert werden. In der französischen Kohorte mit mehr als 900 SpA-Patienten zeigte sich eine Komplikationsrate von 11,7\% [81]. Die ophthalmologische Therapie besteht aus der Gabe von topischen Steroiden und Mydriatika (Scopolamin). Peri- und intraokuläre Kortikosteroide und meist hoch dosierte orale Kortikosteroide sind ebenfalls effektiv. NSAR und Methotrexat (MTX) haben nur einen begrenzten Stellenwert. Für das Medikament Sulfasalazin („disease-modyfying antirheumatic drug" [DMARD]) gibt es einige Studien, die eine vorbeugende Wirksamkeit belegen [87, 88]. Die Datenlage ist hierzu aber begrenzt. Bei Patienten, die nicht auf die Standardtherapie der AAU ansprechen, kann eine Therapie mit Tumor-Nekrose-Faktor-Inhibitoren (TNFi) in Erwägung gezogen werden. Adalimumab ist für die Behandlung der nichtinfektiösen posterioren oder intermediaten Uveitis zugelassen [89]. Kontrollierte Studien für die anteriore Uveitis liegen nicht vor. Unter einer Behandlung mit TNFi verringert sich die Inzidenz von Uveitisschüben verglichen mit Plazebo (Plazebo 15,6/100 Patientenjahre; Infliximab 3,4/100 Patientenjahre; Etanercept 7,9/100 Patientenjahre; $p=0,05$ ) [90]. Adalimumab oder Infliximab sind im Vergleich zum Fusionsprotein Etanercept effektiver in der Verhinderung neuer Schübe oder einer Erstmanifestation einer AAU bei Patienten mit axSpA [91-94]. Bei retrospektiven Studien mit Sulfasalazin als aktivem Vergleichspräparat unterschied sich die Uveitisrate im Vergleich zu Etanercept nicht (Etanercept 10,7 [95\%-CI 5,5-17,6], Sulfasalazin
14,7 [95\%-CI 6,4-26,5]) [91]. Eine offene Studie mit Adalimumab zeigte, dass sich die Schubhäufigkeit einer AAU unter Therapie mit Adalimumab verglichen mit Plazebo um ca. $50 \%$ senken ließ [95]. Daten zu Certolizumab und Golimumab in Bezug auf AAU liegen nicht vor. Die Studien mit Secukinumab (IL17-Blocker) zeigten keine Wirksamkeit bei nichtinfektiöser Uveitis [96, 97].

\subsubsection{Gastrointestinale Beteiligung}

Bis zu $60 \%$ der Patienten mit AS zeigen histologische Zeichen einer Darmentzündung [98] - zumeist ohne klinische Symptome. Ein kleiner Teil dieser Patienten entwickelt mit der Zeit das Vollbild einer CED (entweder einen Morbus Crohn [MC] oder eine Colitis ulcerosa [CU]) [99]. Insgesamt leiden etwa $10 \%$ der Patienten mit AS gleichzeitig an einer CED [73]. Andererseits gaben $40 \%$ der Patienten in einer CED-Ambulanz muskuloskeletale Beschwerden an [100]. In einer belgischen CED-Kohorte, in der alle 251 Patienten eine Röntgenuntersuchung der SI-Gelenke unabhängig von Rückenschmerzen erhielten, wurde bei $27 \%$ eine radiologische Sakroiliitis gefunden [101]. In einer Schweizer CEDKohorte mit 950 Patienten betrug die Prävalenz der AS nur $2 \%$ bei UC bzw. $6 \%$ bei MC, aber die der peripheren Arthritis $21 \%$ bei UC und $33 \%$ bei MC [102]. Die effektive Behandlung der muskuloskeletalen Manifestationen führte bei ASPatienten auch zu einer Besserung der Darmsymptome [98]. Die Behandlung mit einem Coxib führte bei Patienten mit CED zu keiner Verschlechterung der Darmsymptome [103].

Die Standardtherapie der CED besteht aus der Gabe antiinflammatorischer Substanzen wie 5-Aminosalizylsäure oder Kortikosteroiden und Immunsuppressiva wie Azathioprin und TNFi [104]. Etanercept ist bei Patienten mit CED unwirksam [105]. In einer aktuellen Metaanalyse konnte jetzt bestätigt werden, dass die gepoolte CED-Rate unter einer Therapie mit TNFi bei Infliximab (0,2 pro 100 Patientenjahre) und Adalimumab (0,6 pro 100 Patientenjahre) gegenüber Etanercept (2,2 pro $100 \mathrm{~Pa}$ tientenjahre) niedriger ist [92]. Selten kann eine CED als paradoxe Reaktion 
auch unter einer TNFi-Therapie neu auftreten [106]. Secukinumab als IL-17Blocker zeigte keine Wirksamkeit in der Behandlung eines Morbus Crohn [107]. Die Behandlung der CED liegt in der Hand des Gastroenterologen und sollte darüber hinaus ggf. in Kooperation mit dem Rheumatologen erfolgen.

\subsubsection{Beteiligung der Haut}

Patienten mit SpA haben nicht selten psoriasiforme Hautläsionen. Bis zu 20\% der Patienten mit AS leiden zusätzlich an einer Psoriasis vulgaris. In der deutschen Inzeptionskohorte GESPIC hatten 10-15\% der Patienten mit AS zusätzlich eine Psoriasis vulgaris [74]. Patienten mit Nagelbeteiligung bei Psoriasis haben tendenziell häufiger eine Gelenkentzündung [74].

\subsubsection{Weitere betroffene Organsysteme}

Herz: Patienten mit AS können als kardiale Manifestationen Reizleitungsstörungen, eine Aortitis und/oder eine Aorteninsuffizienz entwickeln [108, 109]. Die Prävalenz für Reizleitungsstörungen schwankt zwischen 1 und $33 \%$ und für die Aorteninsuffizienz zwischen 1 und $10 \%$. In einer aktuellen Analyse aus dem schwedischen Patientenregister $(n=27.700$ Patienten mit SpA) (NPR) bestätigte sich das erhöhte Risiko für Herzrhythmusstörungen und Aortenklappeninsuffizienz (HR für AVBlock: 2,3 [95\%-CI 1,6-3,3], HR für Vorhofflimmern: 1,3 [95\%-CI 1,2-1,6], HR für Schrittmacher: 2,1 [95\%-CI 1,6-2,8] und HR für Regurgitation der Aorta: 1,9 [95\%-CI 1,3-2,9]) [110].

Lunge: Restriktive Ventilationsstörungen liegen vornehmlich bei Patienten mit fortgeschrittener AS als Resultat der reduzierten Thoraxbeweglichkeit vor. Zum Teil liegen dabei auch parenchymatöse Veränderungen vor [111-113].

Niere: Daten zur Beteiligung der Niere stützen sich überwiegend auf einzelne Kohortenstudien und Fallberichte. Es wird überwiegend über Amyloidose berichtet, wobei die Häufigkeit in einer kleinen Fallserie mit $7 \%$ angegeben wird [114]. Die Inzidenz von renalen Auffälligkeiten schwankt zwischen 10 und $35 \%$ $[114,115]$. Patienten mit AS haben ein erhöhtes Risiko für die Entwicklung einer Nephrolithiasis [116]. In dieser schwedischen Kohortenstudie konnte als Risikofaktoren das Vorhandensein einer CED sowie einer Nephrolithiasis in der Vorgeschichte herausgearbeitet werden.

\subsection{Komorbidität und Mortalität}

Bei Patienten mit SpA können Komorbiditäten vorliegen und sollten regelmäBig diagnostiziert und therapiert werden. In der internationalen COMOSPA-Studie zur Erfassung von Komorbiditäten und ihrer Screeningstrategie bei Patienten mit SpA waren die häufigsten Komorbiditäten Osteoporose (13\%), gastrointestinale Ulzerationen (11\%) und kardiovaskuläre Erkrankungen (3,5\%) [117]. Als häufigster Risikofaktor für Komorbiditäten zeigten sich in dieser Studie eine arterielle Hypertonie (34\%), Rauchen $(29 \%)$ und eine Hypercholesterinämie (27\%).

Die 2 wichtigsten Komorbiditäten bei Patienten mit SpA sind die kardiovaskulären Erkrankungen und die Veränderung der Knochendichte im Sinne von Osteoporose und Osteopenie. Da es sich bei der Beteiligung der Knochen um eine Folgeerkrankung mit muskuloskeletaler Symptomatik handelt, wird letztere Problematik im Abschn. 3.1.1.4 besprochen.

Eine Vielzahl weiterer Symptome und Komorbiditäten wurde bei Patienten mit SpA beschrieben.

Vitamin-D-Mangel: In einer Untersuchung der DESIR-Kohorte konnte gezeigt werden, dass ein Vitamin-D-Mangel mit einer hohen Krankheitsaktivität und einer Adipositas assoziiert ist [118].

Die Rate an Depression ist bei Patienten mit axSpA erhöht (Frauen 1,81 [95\%CI 1,44-2,24] und Männer 1,49 [95\%-CI 1,20-1,89]) [119-121]. Zudem sind Patienten mit Depression schwieriger analgetisch einzustellen. Daten aus der amerikanischen SpA-Kohorte PSOAS zeigen, dass 9,5\% der Patienten in der Kohorte dauerhaft Opioide einnahmen und diese Patienten auch einen höheren Score in einem Depressionsfragebogen (Center for Epidemiologic Studies Depression Scale [CES-D]) aufwiesen [122].

Adipositas: Auch wenn sich der mittlere BMI bei Patienten in einer niederlän- dischen Fall-Kontroll-Studie nicht signifikant von dem der gesunden Vergleichspopulation unterschied, lagen bei axSpA-Patienten mit Adipositas eine höhere Krankheitsaktivität und eine schlechtere körperliche Funktionsfähigkeit vor als bei normgewichtigen axSpA-Patienten [123]. Patienten mit Adipositas sprechen auf eine TNFi-Therapie deutlich schlechter als normgewichtige Patienten an (s. Abschn. 8 Therapie).

Fibromyalgie: Chronifizierte Schmerzen können bei Patienten mit axSpA auftreten. Die Angaben zur Prävalenz der Fibromyalgie schwanken zwischen 20 und $24 \%$ [124-126]. Symptome eines generalisierten Schmerzsyndroms werden häufiger von AS als von Patienten mit nraxSpA geklagt (29\% in AS und 19\% in nr-axSpA) [124], Patienten mit axSpA und FM zeigen eine niedrigere Ansprechrate auf eine TNFi-Therapie als $\mathrm{Pa}$ tienten ohne generalisiertes Schmerzsyndrom [125].

Sexuelle Dysfunktion: In einer kleinen Fall-Kontroll-Studie wird eine im Vergleich zum Gesunden höhere Rate an sexueller Dysfunktion bzw. Symptomen des unteren Harntraktes beschrieben [121]. Weitere Studien, die die Ein- und Ausschlusskriterien erfüllt hätten, wurden allerdings nicht identifiziert.

\subsubsection{Kardiovaskuläres Risiko}

Als wesentlicher Faktor für akzelerierte Arteriosklerose ist die unbehandelte systemische Entzündung, wie sie auch bei Patienten mit AS vorliegen kann, identifiziert worden. Bei AS-Patienten liegt eine um $20-40 \%$ erhöhte kardiovaskuläre Mortalität vor [127]. Eine kanadische Fall-Kontroll-Studie mit 21.473 Patienten mit AS berechnete die vaskuläre Mortalität mit einer Hazard Ratio (HR) von 1,36 (95\%-CI 1,13-1,65), wobei die zerebrovaskuläre Mortalität mit einer HR von 1,60 (95\%-CI 1,17-2,2) und die kardiovaskuläre Mortalität mit einer HR von 1,35 (95\%-CI 1,07-1,70) berechnet wurde [128]. AS-Patienten haben verglichen mit Kontrollen eine höhere Arterioskleroseprävalenz, wobei die Breite der Intima-media-Verdickung mit der Höhe der Krankheitsaktivität assoziiert ist [127, 129-131]. Einzelne Studien konnten jedoch keinen Unterschied in der Ausprä- 
gung der subklinischen Arteriosklerose bei Patienten mit AS im Vergleich zur gesunden Normalbevölkerung identifizieren [132-134]. Das erhöhte kardiovaskuläre Risiko konnte in der großen Studie des schwedischen Patientenregisters bestätigt werden [110, 135]. Die Herzinfarktprävalenz von Patienten mit AS war bei einer niederländischen Patientenbefragung mit $4,4 \%$ im Vergleich zu $1,2 \%$ in der niederländischen Bevölkerung erhöht [136]. Die alters- und geschlechtsadjustierte OR, als AS-Patient einen Herzinfarkt zu erleiden, lag bei 3,1 (95\%-CI 1,9-5,1). Eine Metaanalyse zeigte dagegen keine Erhöhung der Herzinfarktinzidenz (,risk ratio“ 1,88 ; $95 \%$ CI 0,83-4,28). Das Risiko, einen Schlaganfall zu erleiden, war in dieser Analyse ebenfalls nicht erhöht [137]. In einer kleinen chinesischen Fall-KontrollStudie konnten zwischen AS-Patienten und gesunden Kontrollen keine Unterschiede in der Herzfunktion (echokardiographisch ermittelt) gezeigt werden [131]. Eine hohe Krankheitsaktivität war bei AS-Patienten mit einer Verschlechterung des Lipidprofils assoziiert [138]. Eine weitere Studie mit Fokus auf Serumparameter wie ,asymmetric dimethylarginen“(ADMA)-Spiegel bestätigt die eingeschränkte Endothelfunktion [139].

Die antientzündliche Wirkung einer Therapie mit TNFi hat möglicherweise auch Einfluss auf das kardiovaskuläre Risiko. Zwei unkontrollierte Studien zeigen unter einer TNFi-Therapie ein verbessertes Lipidprofil [140, 141]. Unter Etanercept wurde bei Patienten mit AS eine verbesserte mikrovaskuläre Funktion festgestellt [142]. Die EULAR (European League Against Rheumatism) hat für Patienten mit Arthritis evidenzbasierte Empfehlungen zum Management des kardiovaskulären Risikos herausgegeben, die uneingeschränkt auch für Patienten mit axSpA gelten [143].

\subsubsection{Müdigkeit}

Ein wesentlicher Faktor der Morbidität bei Patienten mit entzündlich rheumatischen Erkrankungen ist die oft als belastend empfundene Müdigkeit, die nach der Klage über Schmerzen und Steifigkeit als dritthäufigstes Symptom von den Patienten genannt wird [144]. Die Fatigue-
Symptomatik tritt signifikant häufiger bei AS-Patienten als bei gesunden Kontrollen auf [145]. Aus pathophysiologischen Überlegungen heraus kann die Fatigue dem gestörten Nachtschlaf aufgrund von Schmerzen oder aufgrund der chronischen Entzündung und der daraus resultierenden Erschöpfung zugeordnet werden. Die Angaben zur Prävalenz der Müdigkeit und Abgeschlagenheit schwanken zwischen 53 und $68 \%$, wobei verschiedene Definitionen als auch verschiedene Schweregradeinteilungen zur Erfassung der Prävalenz verwendet wurden [145-148]. Das Ausmaß der Müdigkeit ist mit der Höhe der Krankheitsaktivität und der psychischen Gesundheit (gemessen mit SF-36) assoziiert $[149,150]$. Die Prävalenz der Müdigkeit ist bei Frauen im Vergleich zu Männern erhöht und kann auch schon früh im Krankheitsverlauf als limitierender Faktor auftreten [38, 144, 149, 151]. Im klinischen Alltag ist es am sinnvollsten, für die Quantifizierung der Müdigkeit (Fatigue) die 1. Frage des BASDAI zu verwenden (s. Abschn. 7.1.1) [144]. Wie auch schon in interventionellen Studien gezeigt, konnte in einer aktuellen Kohortenstudie erneut gezeigt werden, dass sich die Anzahl der Patienten mit schwerer Fatigue-Symptomatik unter einer TNFi-Therapie signifikant reduzieren lässt $(87,8 \%$ vs. $72,7 \%, p<0,0001)$ $[148,152,153]$. Der Einfluss einer Fatigue-Symptomatik auf die Arbeitsproduktivität ist naheliegend und konnte auf Gruppenebene auch bei AS-Patienten gezeigt werden [154].

Der gestörte Nachtschlaf kann durch Rückenschmerzen entstehen, aber auch durch Depressionen oder Ängste ausgelöst werden. In einer chinesischen FallKontroll-Studie zeigte sich eine reduzierte Schlafqualität bei $35,4 \%$ der AS-Patienten im Gegensatz zu 22,9\% der Kontrollpatienten [120].

\subsubsection{Mortalität}

Die standardisierte Mortalitätsrate (SMR) liegt bei AS-Patienten zwischen 1,33 und 1,8 [155-157]. In einer aktuellen FallKontroll-Studie aus Schweden wird die Mortalität mit einer HR von 1,60 (95\%CI 1,44-1,77) angegeben [157]. In dieser Kohorte zeigte sich ein höheres Mortalitätsrisiko für Frauen $(\mathrm{HR}=1,83$;
95\%-CI 1,50-2,22) gegenüber Männern $(\mathrm{HR}=1,53$; 95\%-CI 1,36-1,72). Komorbiditäten, Hüft-TEP-Implantation und ein niedriger Bildungsstandard wurden als Prädiktoren für Tod identifiziert. In einer britischen Fall-Kontroll-Studie zeigte sich, dass Patienten, die neu mit Statinen behandelt wurden, ein höheres Überleben zeigten als Patienten ohne Statintherapie (HR 0,63 [95\%CI 0,46-0,85]) [158]. Aktuelle schwedische Registerdaten mit 677 AS-Patienten zeigten bei einer SMR von 1,61 (95\%CI 1,29-1,93) einen Unterschied zwischen den Geschlechtern (männlich 1,63 vs. weiblich $1,38, p<0,001$ ) [159]. Die Haupttodesursache waren HerzKreislauf-Erkrankungen ( $40 \%)$, gefolgt von malignen Erkrankungen (26,8\%) und Infektionen (23,2\%). Die höhere Mortalität bei Männern konnte in der kanadischen Fall-Kontroll-Studie bestätigt werden (HR für Männer vs. Frauen $1,82$ [95\%-CI $1,33-2,48 ; p<0,001]$ ). Folgende Faktoren waren mit einer reduzierten Überlebensrate assoziiert: Diagnoseverzögerung (OR 1,05), erhöhtes C-reaktives Protein (CRP) (OR 2,68), Arbeitsunfähigkeit (OR 3,65) und der Umstand, dass keine NSAR-Therapie durchgeführt wurde (OR 4,35). Die Reduktion der Mortalität bei Gebrauch von NSAR (HR 0,1 [95\%-CI 0-0,61, $p=0,01])$ zeigte sich auch in der kanadischen Fall-Kontroll-Studie, die zudem eine Risikoreduktion durch Statine zeigte (HR 0,25 [95\%-CI 0,13-0,51, $p<0,001]$ ) [128]. Die Lebenserwartung ist bei weniger stark betroffenen Patienten mit SpA wohl nicht erheblich vermindert. Schwer betroffene AS-Patienten haben aber ein erhöhtes Risiko, früher zu versterben vor allem an Herz-Kreislauf-, Nierenund Atemwegserkrankungen sowie an Amyloidose [160].

Die Mortalität bei hospitalisierten ASPatienten ist hauptsächlich durch eine vorliegende HWS-Fraktur und begleitende Rückenmarkverletzung (OR 13,43 [95\%-CI 8,00-22,55]) bzw. durch eine Sepsis (OR 7,63 [95\%-CI 5,62-10,36]) erhöht [161].

Eine deutsche Untersuchung an ASPatienten, die in den 40er- bis 70er-Jahren eine Radiumtherapie erhalten hatten, zeigte ein erhöhtes Risiko für akute mye- 
Nicht-röntgenologisches Stadium
Röntgenologisches Stadium

\section{Modifizierte New-York-Kriterien 1984}

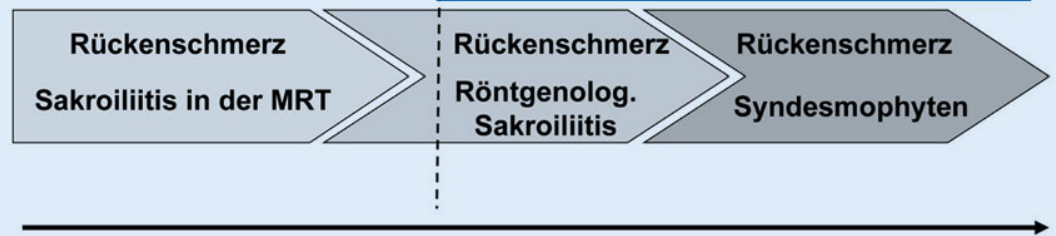

Zeit (Jahre)

Abb. 1 \ Verlauf der axialen Spondyloarthritis [171]. ( ${ }^{*}$ Die modifizierten New-York-Kriterien dienen zur Charakterisierung des röntgenologischen Stadiums und werden im Abschn. 4.2 und 6.1.1.1 näher erläutert). (Publikation mit Genehmigung durch ASAS, www.asas-group.org)

loische Leukämie: Es wurden 11 Fälle berichtet, es waren aber nur 2,9 Fälle erwartet worden $(p<0,001)$ [162].

\section{Empfehlung/Statement}

\section{3-5}

Extraskeletale Manifestationen und Begleiterkrankungen, wie z. B. gastrointestinale und/oder kardiovaskuläre Erkrankungen, bzw. deren Risikofaktoren sollten regelmäßig evaluiert und therapiert werden.

Empfehlungsgrad: $B$

Evidenz: 3b

\section{Klassifikations- und Diagnosekriterien}

\section{Schlüsselfrage 3}

- Was sind die wichtigsten Symptome/ Befunde/Kriterien bei Patienten mit chronischen Rückenschmerzen, die zur Diagnose SpA führen?

Ein Teil dieser Schlüsselfrage wird in anderen Schlüsselfragen beantwortet. Die Frage nach den Symptomen und Befunden wird im Abschn. 3.1 (muskuloskeletale Beschwerden) und im Abschn. 3.2 (extraskeletale Manifestation) erläutert. Daher wird in diesem Teil der Schlüsselfrage nur auf die Klassifikationskriterien eingegangen.

\subsection{ASAS(Assessment of SpondyloArthritis international Society)-Klassifikationskriterien}

In Ablösung der ESSG(European Spondylarthropathy Study Group)- und Amor-Kriterien wurden 2009 die ASASKlassifikationskriterien für axiale und periphere SpA publiziert [2, 163-165]. Auf die Klassifikation und Diagnostik der peripheren $\mathrm{SpA}$ wird in der vorliegenden Leitlinie nicht eingegangen, da sich die Leitlinie auf die axiale SpA konzentriert. Die Diagnostik und Therapie von unspezifischen Kreuzschmerzen wird in der Nationalen Versorgungsleitlinie (NVL) "Nicht spezifischer Kreuzschmerz" thematisiert [166].

Die ASAS-Kriterien erfordern als Eingangskriterium die Präsenz von chronischen Rückenschmerzen $\geq 3$ Monate und ein Patientenalter von $<45$ Jahren bei Beginn der Symptomatik. Wenn dieses Eingangskriterium bei einem Patienten vorliegt, kann die weitere Abklärung der Verdachtsdiagnose axiale SpA anhand eines klinischen Armes (HLAB27 plus $\geq 2$ weitere SpA-Kriterien) oder eines bildgebenden Armes (Sakroiliitis in der Bildgebung plus $\geq 1$ weiteres SpAKriterium) erfolgen (• Abb. 1 und 2). Im Abschn. 5.2.1 wird auf den diagnostischen Nutzen der einzelnen SpA-Parameter eingegangen. Die Kriterien weisen eine Sensitivität von 82,9 und eine Spezifität von $84,4 \%$ auf, wenn das gesamte Set getestet wurde. Wenn nur der bildgebende Arm alleine getestet wurde, liegen eine Sensitivität von $66,2 \%$ und eine Spezifi- tät von 97,3\% vor [2, 167]. Die Spezifität ist damit für die ASAS-Klassifikationskriterien besser als für die Amor- und ESSG-Kriterien (• Tab.4). Inzwischen ist auch die Validität der ASAS-Klassifikationskriterien mittels einer Längsschnittuntersuchung bestätigt worden sowie die Validität der ASAS-Klassifikationskriterien in der Routineversorgung in einer unabhängigen Kohorte bestätigt worden $[168,169]$. Der positive prädikative Wert (PPV) lag in der Längsschnittuntersuchung bei 92,2\% (axSpA 93,3) und der negative prädikative Wert bei $62,0 \%$. In der französischen DESIR-Kohorte konnte zudem die externe Validität des klinischen und des Bildgebungsarms gezeigt werden [170].

Theoretisch kann zwischen der NVL Nicht spezifischer Kreuzschmerz und den ASAS-Klassifikationskriterien für axiale SpA eine Diskrepanz bezüglich der Länge der klinischen Symptomatik bestehen. Die NVL Nicht spezifischer Kreuzschmerz empfiehlt eine Diagnostik ab einem Zeitintervall von 6 Wochen, wohingegen die ASAS-Klassifikationskriterien für axiale $\mathrm{SpA}$ als Eingangsvoraussetzung einen Rückenschmerz von 12 Wochen erfordern. Der Schwellenwert von 12 Wochen ist arbiträr gewählt worden, weil bei kurzer Krankheitsdauer nichtentzündliche Ursachen wahrscheinlicher als entzündliche Ursachen sind. Die Diskrepanz von 6 Wochen zwischen NVL und ASASKlassifikationskriterien ist nach Ansicht der Autoren der vorliegenden Leitlinie lediglich eine theoretische Lücke, da sich die Diagnoseverzögerung für eine axiale SpA eher in Monaten und Jahren als in Wochen bemisst.

Die ASAS-Klassifikationskriterien dienen zur Klassifikation von Patienten und sind NICHT primär für die Diagnosestellung für diese Patienten entwickelt worden ( $\bullet$ Abb. 2). Die Kriterien ermöglichen die Durchführung von Studien. Rheumatologen können die Klassifikationskriterien aber nichtsdestoweniger zur Bestätigung ihrer Diagnose heranziehen. Da die Sensitivität dieser Kriterien aber "nur" etwas höher als $80 \%$ ist, ist davon auszugehen, dass es SpA-Patienten gibt, die die Erkrankung haben - ohne dass sie die genannten Kriterien erfül- 
Patienten mit chron. Rückenschmerzen $\geq 3$ Monate, bei Beginn < 45 Jahre

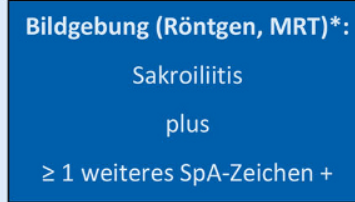

oder

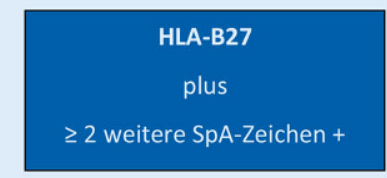

* Sakroiliitis in der Bildgebung:

- Aktive (akute) Entzündung in der MRT, gut vereinbar mit einer SpAassoziierten Sakroiliitis

oder

- Definitive röntgenologische Sakroiliitis (Strukturveränderungen) gemäß

+ zusätzliche SpA-Zeichen

- entzündlicher Rückenschmerz

- Arthritis

- Enthesitis (Ferse)

- anteriore Uveitis

- Daktylitis

- Psoriasis

- Kolitis bei CED

- Gutes Ansprechen auf NSAR

- Familiengeschichte für $S p A$

- HLA-B27+

- erhöhtes CRP den mod. NY-Kriterien

nichtröntgenologische axiale SpA (nraxSpA), die letztere als AS bezeichnet. ${ }^{1}$

Die ASAS-Klassifikationskriterien für die axiale SpA dienen der Kategorisierung von Patienten in Studien. Die klinische Diagnose kann auf die Präsenz von SpA-Variablen, wie sie in den Klassifikationskriterien benannt sind, beruhen, die Diagnosestellung umfasst aber immer eine klinische Bewertung des Krankheitsbildes. Je mehr SpA-Parameter bei einem Patienten vorliegen, umso eher wird auch die Diagnose einer axialen SpA gestellt werden, wie dies z. B. in der niederländischen Frühkohorte SPACE gezeigt werden konnte [172]. In demselben Kollektiv untersuchten die niederländischen Kollegen auch die Frage, welche der SpA-Variablen zur Diagnosestellung einer SpA beitragen, wenn bei Patienten keine oder nur eine SpA-Variable vorliegt [173]. Als wichtige SpA-Variablen wurde in dieser Studie das Vorhandensein von einer Sakroiliitis bzw. einem positiven HLAB27-Befund identifiziert. In der spanischen ESPERANZA-Kohorte konnte die Bedeutung des Vorhandenseins einer Sakroiliitis unterstrichen werden [174]. Der Nachweis einer Sakroiliitis in der MRT (LR 6,6) oder Sakroiliitis im konventionellen Röntgenbild (pos. LR 31,3) sowie peripherer Arthritis (pos. LR 8,9) wies eine hohe diagnostische Aussagekraft auf. Eine geringe diagnostische Aussagekraft wies jedoch die SpA-Variable familiäre Disposition (pos. LR 1,5), gutes Ansprechen auf NSAR (pos. LR 1,6), entzündlicher Rückenschmerz (pos. LR 2,3) und HLA-B27 (pos. LR 2,8) auf. In dieser Ko-

len (ca. 20\%). Dies sollte aber immer fachärztlich begründet werden. Da die Spezifität ebenfalls nur $80-85 \%$ beträgt, wird es auch Patienten geben, die die Klassifikationskriterien formal erfüllen, die aber keine axiale SpA haben.

Die ASAS-Klassifikationskriterien sind für die gesamte Gruppe der Patienten mit axialer SpA entwickelt worden, d.h. in dieser Gruppe sind sowohl Patienten ohne strukturelle Veränderungen als auch Patienten mit strukturellen Veränderungen in den SI-Gelenken eingeschlossen. Die erstere Gruppe wird als

\footnotetext{
1 Die Mehrzahl der in dieser Leitlinie genannten Studien bezieht sich auf Patienten mit AS. Gerade in jüngster Zeit sind aber auch viele Studien, die Patienten mit nr-axSpA umfassen, publiziert worden. Im klinischen Alltag hat diese artifizielle Einteilung nur eine untergeordnete Bedeutung und spielt im Wesentlichen eine Rolle bei der Indikation für eine Biologikatherapie. Die überwiegende Anzahl der medikamentösen Therapien ist in Deutschland für Patienten mit AS. Seit 2012 sind bis auf Infliximab alle TNFi für die Behandlung symptomatischer Patienten mit nr-axSpA und objektivem Entzündungsnachweis* zugelassen (Abschn. 8.4.2) (*Die modifizierten New-York-Kriterien dienen zur Charakterisierung des röntgenologischen Stadiums und werden im Abschn. 4.2 und 6.1.1.1 nähererläutert).
} 
Tab.5 Klassifikationskriterien für die

ankylosierende Spondylitis (New York,

1984)

Klinische Parameter

- Entzündlicher Rückenschmerz

- Limitation der Wirbelsäulenbeweglichkeit in 3 Ebenen

- Einschränkung der Thoraxexkursionsfähigkeit

Radiologische Parameter

Sakroiliitis mindestens:

- Bilateral Grad II

- Unilateral Grad III oder IV

horte waren allerdings auch nur $48 \%$ der Patienten HLA-B27-positiv.

\subsection{Modifizierte New-York- Kriterien}

Die auf der Basis der modifizierten NewYork-Kriterien vorgenommene Differenzierung zwischen den verschiedenen Ausprägungen von strukturellen Veränderungen der SI-Gelenke beruht auf dem Schweregrad der röntgenologischen Veränderungen in diesen Gelenken und berücksichtigt darüber hinaus auch klinische Parameter (• Tab. 5; [175]). Für die Diagnose einer AS ist ein Grad $\geq 2$ beidseits oder $\geq 3$ unilateral gefordert (s. Abschn. 6.1.1.1).

Die Unterscheidung zwischen Patienten mit (= AS) und ohne (=nr-axSpA) nativ-röntgenologisch sichtbaren strukturellen Veränderungen in den SI-Gelenken ist primär eine historische Unterteilung, die zum Teil auch zulassungstechnische Aspekte hat. Zur Beschreibung des Schweregrades der Erkrankung können die genannten Kriterien begrenzt hilfreich sein. In der klinischen Präsentation unterscheiden sich Patienten mit AS und nr-axSpA nur hinsichtlich des Ausmaßes des strukturellen Schadens und der Entzündungsaktivität, jedoch nicht hinsichtlich subjektiver Parameter wie Schmerz, Funktionseinschränkung und anderer patientenberichteter Endpunkte (siehe auch - Abb. 1; [75, 176, 177]). Einzelne Studien zeigen jedoch auch eine stärkere Beeinträchtigung der nr-axSpAPatienten [178].

\section{Empfehlung/Statement}

\section{4-1}

Die Diagnosestellung einer axialen SpA soll aufgrund von Anamnese, klinischer Untersuchung, Laborbefunden, Bildgebung und unter Berücksichtigung von Differenzialdiagnosen erfolgen. Empfehlungsgrad: Evidenz: Expertenkonsens

\section{4-2}

Die ASAS-Klassifikationskriterien beinhalten wichtige Parameter der axialen SpA, können die oben beschriebene Diagnosefindung aber nicht ersetzen. Empfehlungsgrad: Statement Evidenz: -

\section{Erstdiagnose/ Überweisungsstrategie}

\section{Schlüsselfrage 4}

- Welches Ausmaß an Labor/Röntgen ist in der Primärversorgung vernünftig?

\subsection{Rationale und Ziele für eine verbesserte Frühdiagnostik}

Unter den rheumatischen Erkrankungen zeichnet sich die AS durch eine lange Zeitdauer zwischen Symptombeginn und Diagnosestellung aus. Die mittlere Zeit der Diagnoseverzögerung schwankt und beträgt zwischen 5 und 14 Jahren $[16,17]$. Dies ist vor allem darauf zurückzuführen, dass nicht ein einzelnes Symptom wegweisend für die Diagnose ist, sondern dass die relativ wenigen betroffenen Patienten aus der großen Gruppe der Patienten mit unspezifischen Kreuz- oder Rückenschmerzen möglichst optimal selektiert werden müssen. Das Ziel der vorliegenden Leitlinie ist es deshalb, möglichst evidenzbasierte, aber auch praktikable Empfehlungen für die Frühdiagnostik bzw. die Überweisung in der Primärversorgung zu geben und die Häufigkeit von Diagnoseverzögerungen zu minimieren. Die Praktikabilität soll dadurch gewährleistet werden, dass die zu erhebenden Variablen einfach zu verwenden sind, dass sie sicher als auffällig oder unauffällig zu identifizieren sind und dass die Auswertung der Variablen zuverlässige Ergebnisse erzielt [179].

Das Ziel einer frühen Diagnosestellung bei Patienten mit axialer SpA ist die rechtzeitige Sicherstellung einer optimalen Therapie und die Vermeidung unnötiger weiterer Diagnostik und nicht indizierter Therapie. Grundsätzliches Ziel ist auch die Verhinderung struktureller Schäden, das bedeutet Vermeidung von Funktionseinbußen und von Knochenneubildung. In diesem Zusammenhang ist darauf hinzuweisen, dass das Auftreten struktureller Schäden bei Patienten mit axialer SpA variabel ist. In einer Kohorte von Patienten mit entzündlichem Rückenschmerz, die kürzer als 2 Jahre bestanden, hatten $20 \%$ der Patienten bereits strukturelle Veränderungen in den SI-Gelenken [180]. Es ist darauf hinzuweisen, dass zwei Drittel der Patienten mit AS nicht fachrheumatologisch, sondern allgemeinmedizinisch versorgt werden. Dies konnte unabhängig voneinander in einem schottischen und einem USamerikanischen Register gezeigt werden [181, 182].

\subsection{Untersuchte Variablen}

Die getesteten Variablen und die untersuchten Populationen unterscheiden sich in den einzelnen Studien. Klinische Variablen wurden allein oder in Kombination mit HLA-B27 und/oder bildgebender Diagnostik getestet. Klinische Variablen, die durch einfache Befragung der Patienten erhoben wurden, sind am häufigsten untersucht worden. Keine der getesteten Variablen ist als Einzelparameter zu empfehlen, da Sensitivität und Spezifität nicht ausreichen, um eine Frühdiagnostik praktikabel zu gestalten. Die Erhebung von mehreren Variablen (sog. Sets) erhöht die Aussagekraft deutlich. Die Leitlinien-Autoren stellen in diesem Kontext aber klar, dass das alleinige Erfüllen von Klassifikationskriterien NICHT die Diagnose einer axialen SpA sichern kann.

Für die Bewertung der einzelnen Variablen sind Sensitivität, Spezifität und LR von besonderer Bedeutung. Die LR gibt an, um wie viel Mal häufiger ein positives Testresultat bei Personen mit Erkrankung im Vergleich zu Personen 


\begin{tabular}{|c|c|c|c|c|c|c|c|c|}
\hline Parameter & $\begin{array}{l}\text { Sensiti- } \\
\text { vität (\%) }\end{array}$ & $\begin{array}{l}\text { Spezifität } \\
(\%)\end{array}$ & $\begin{array}{l}\text { Likelihood- } \\
\text { Ratio (LR) }\end{array}$ & $\begin{array}{l}\text { Nachtest- } \\
\text { wahrschein- } \\
\text { lichkeit (\%) }\end{array}$ & $\begin{array}{l}\text { Anzahl Patienten, die vom } \\
\text { Rheumatologen gesehen } \\
\text { werden müssen, um bei ei- } \\
\text { nem Patienten die Diagnose } \\
\text { axiale SpA zu stellen }\end{array}$ & $\begin{array}{l}\text { Test- } \\
\text { kosten }\end{array}$ & $\begin{array}{l}\text { Auswer- } \\
\text { tung }\end{array}$ & $\begin{array}{l}\text { Gesamt- } \\
\text { beurtei- } \\
\text { lung }\end{array}$ \\
\hline \multicolumn{9}{|l|}{ Klinische Parameter } \\
\hline $\begin{array}{l}\text { Entzündlicher } \\
\text { Rückenschmerz }\end{array}$ & $69-75$ & $75-80$ & 3 & 14 & 7 & Gering & Gut & ++ \\
\hline $\begin{array}{l}\text { Ansprechen auf } \\
\text { NSAR-Therapie }\end{array}$ & $61-93$ & $48-85$ & $1,8-5,1$ & 21 & 5 & Gering & Gut & + \\
\hline Enthesitis & $15-37$ & $89-94$ & 3,4 & - & - & - & - & - \\
\hline Uveitis & $10-22$ & $97-99$ & 7,3 & 28 & $3-4$ & Gering & Gut & 0 \\
\hline Periphere Arthritis & $40-62$ & $90-98$ & 4,0 & - & - & - & - & - \\
\hline $\begin{array}{l}\text { Anamnese einer } \\
\text { peripheren Arthritis }\end{array}$ & 10,6 & 95,7 & 2,5 & - & - & - & - & - \\
\hline Familienanamnese & 25 & 96 & 6,4 & 25 & 4 & Gering & Moderat & 0 \\
\hline \multicolumn{9}{|l|}{ Laborparameter } \\
\hline HLA-B27 & $83-96$ & $90-96$ & 9,0 & 32 & 3 & Moderat & Sehr gut & +++ \\
\hline BSG/CRP & $38-69$ & $67-80$ & 2,5 & 11,6 & 10 & Moderat & Gut & 0 \\
\hline \multicolumn{9}{|l|}{ Bildgebung } \\
\hline MRT (Sakroiliitis) & 90 & 90 & 9,0 & 32 & 3 & Hoch & Moderat & + \\
\hline Röntgen (Sakroiliitis) & 80 & 80 & 4 & 17,4 & 5 & Moderat & Moderat & + \\
\hline
\end{tabular}

ohne Erkrankung vorkommt. Die Vortestwahrscheinlichkeit ist die geschätzte Wahrscheinlichkeit, dass ein Patient an einer gewissen Krankheit leidet, bevor Zusatzinformationen aus einem diagnostischen Test vorliegen. Die Vortestwahrscheinlichkeit ergibt sich aus der Prävalenz der Erkrankung unter näher $\mathrm{zu}$ definierenden Umständen der Erhebungsart (z. B. Versorgungsprävalenz vs. Bevölkerungsprävalenz). So unterscheidet sich die Prävalenz in der Bevölkerung von der Prävalenz in einer bestimmten Praxis oder Ambulanz. Anamnestische Daten (z. B. Risikofaktoren) und Befunde der klinischen Untersuchung können ebenfalls eine Rolle spielen. Das heißt, dass die Vortestwahrscheinlichkeit auch dadurch beeinflusst wird, ob die Vorstellung des Patienten im primärärztlichen oder im fachärztlichen Bereich erfolgt. Die Nachtestwahrscheinlichkeit ist die Wahrscheinlichkeit für eine Erkrankung nach Vorliegen von Testergebnissen. Die entsprechenden Parameter sind für die einzelnen Variablen der Abschn. 2.1-2.3 in - Tab. 6 aufgeführt.

\subsubsection{Klinische Variablen}

Mögliche klinische Variablen im Rahmen der Diagnostik sind Fragen nach Art der
Rückenschmerzen, nach anderen Symptomen der SpA, nach der Familienanamnese und nach dem Ansprechen auf eine NSAR-Therapie.

5.2.1.1 Rückenschmerz, entzündlicher Rückenschmerz. Das zentrale Symptom bei Patienten mit axialer SpA ist der chronische Rückenschmerz (>3 Monate) (s. Abschn. 3.1.1.1). Die Prävalenz der AS innerhalb einer Gruppe von Patienten mit chronischen Rückenschmerzen in Allgemeinpraxen wird zwischen 1 und $5 \%$ (China $11 \%$ ) geschätzt [15, 183-185].

Die neuesten Daten zur Prävalenz des entzündlichen Rückenschmerzes in der Allgemeinbevölkerung stammen aus den US-amerikanischen NHANES (National Health and Nutrition Examination Survey) 2009-2010-Daten [186]. Die Prävalenz wird hier mit Werten zwischen 5 und $6 \%$ angegeben (Schwankung beruht auf der Analyse verschiedener Kriteriensets). Als typisches Charakteristikum für die axiale SpA gilt der entzündliche Rückenschmerz (ERS) (s. Abschn. 3.1.1.1). Die Abklärung, ob ein ERS vorliegt oder nicht, setzt eine gewisse Erfahrung bei dem Untersucher voraus. In einer britischen Befragung konnten nur 5\% der Hausärzte im Bezirk Norfolk $(n=186)$ al- le Charakteristika des ERS nennen [187]. Tatsächlich ist bei etwa einem Drittel der Patienten nicht einfach zu entscheiden, ob ein ERS vorliegt oder nicht [179, 188, 189]. Zudem gibt es verschiedene Definitionen des ERS (s. Abschn. 3.1.1.1). In einer deutschen Kohorte von Patienten mit chronischen Rückenschmerzen, die vor dem 45. Lebensjahr begonnen hatten, wurden die einzelnen Variablen für den ERS untersucht [190]. Es konnten Parameter identifiziert werden, die prädiktiv für eine positive Diagnosestellung waren und die in der Primärversorgung ohne apparativen Aufwand erhoben werden können (•Tab. 7). Diese Variablen zeigen eine Sensitivität von $52,2 \%$ und eine Spezifität von $74,6 \%$. Die positive LR liegt bei 2,8 und die negative LR bei 0,64 . Interessanterweise zeigte die Variable "Morgensteifigkeit $>30 \mathrm{~min}^{\text {“ in }}$ dieser Kohorte in der Regressionsanalyse keine signifikanten Unterschiede.

In einer weiteren deutschen Kohorte konnte bei 57,9\% der überwiesenen $\mathrm{Pa}$ tienten die Diagnose einer axialen SpA gestellt werden, wenn ERS in Kombination mit einem positiven HLA-B27-Befund vorlag [188]. Wenn allerdings nur ERS ohne HLA-B27 und ohne bildgebende Hinweise auf eine SpA vorlag, konnte 
Tab. 7 Bildgebende Methoden mit Zielparametern

\begin{tabular}{lllll}
$\begin{array}{l}\text { Bildgebende } \\
\text { Methode }\end{array}$ & Lokalisation & $\begin{array}{l}\text { Entzün- } \\
\text { dung }\end{array}$ & $\begin{array}{l}\text { Knochen- } \\
\text { zerstörung }\end{array}$ & $\begin{array}{l}\text { Knochen- } \\
\text { neubildung }\end{array}$ \\
\hline Röntgen & Axial und peripher & $(-)$ & ++ & +++ \\
\hline MRT & Axial und peripher & +++ & + & + \\
\hline Sonographie & Nur peripher & ++ & ++ & + \\
\hline CT & Überwiegend axial & $(-)$ & +++ & +++ \\
\hline Szintigraphie & Axial und peripher & ++ & - & -
\end{tabular}

die Diagnose axiale SpA nur in 16,2\% der Fälle gesichert werden [191]. Entzündlicher Rückenschmerz ist somit also ein wichtiges Leitsymptom, der diagnostische Wert allein ist jedoch begrenzt.

5.2.1.2 Ansprechen auf NSAR. Patienten mit SpA sprechen im Vergleich zu Patienten mit unspezifischen Rückenschmerzen deutlich besser auf eine NSAR-Therapie an - deshalb ist dies auch als Parameter in die ASAS-Klassifikationskriterien für axiale SpA (s. Abschn. 4) aufgenommen worden [25]. Die NSAR-Variable zeigt eine Sensitivität von $61-93 \%$ und eine Spezifität von $48-85 \%$ bei einer positiven LR von 1,8-5,1. Zur Verwendung dieser Variablen im hausärztlichen Bereich ist aber zu bedenken, dass nicht alle Patienten auch NSAR einnehmen. Es ist zu betonen, dass das Ansprechen auf NSAR nach $24-48 \mathrm{~h}$ vor allem dann gewertet werden kann, wenn die Maximaldosis des entsprechenden NSAR eingenommen wurde.

\subsubsection{Extraspinale Manifestationen.}

Bei Verdacht auf SpA ist es empfehlenswert, die Patienten auf das Vorhandensein bzw. die Anamnese von peripherer Arthritis, Enthesitis (oder Fersenschmerz), Psoriasis vulgaris und anteriorer Uveitis zu befragen. Bei diesen Variablen sollte die niedrige Sensitivität bei allerdings hoher Spezifität (Sensitivität schwankt zwischen 5 und $62 \%$, Spezifität schwankt zwischen 89 und 99\%) grundsätzlich beachtet werden. In einer Untersuchung mit chronischen Rückenschmerzpatienten zeigte sich, dass die Anamnese einer Uveitis eine hohe Wahrscheinlichkeit für die Diagnose einer AS mit sich bringt (OR 7,2) und dass die Anamnese einer Enthesitis (OR 2,7) und einer Psoriasis (OR 3,6) ein erhöhtes Risiko birgt, eine nr-axSpA zu haben [190].

5.2.1.4 Manifestationsalter. Wenn bei Patienten die chronischen Rückenschmerzen im jungen Alter beginnen, ist das Risiko, an einer axialen SpA zu erkranken, erhöht (OR 2,6 [95\%CI 1,5-4,5]) [190]. In den verschiedenen Untersuchungen zur Selektion von hilfreichen Variablen zur Identifizierung von Patienten mit SpA ist das Alter mit unterschiedlichen Grenzwerten festgelegt. Daher schwanken die Altersangaben zwischen 35 und 45 Jahren.

\subsubsection{Variablen Labor}

Als Variablen zur Frühdiagnostik kommt die Bestimmung von HLA-B27 und die quantitative Messung von Akute-PhaseProteinen wie dem C-reaktiven Protein in Betracht.

5.2.2.1 HLA-B27. Bei Verdacht auf SpA ist die Bestimmung von HLA-B27 empfehlenswert. Der Nachweis von HLA-B27 weist eine gute Sensitivität (83-96\%) und eine hohe Spezifität (90-96\%) mit einer positiven LR von 9,0 auf [43]. Zu beachten ist, dass ein positiver HLA-B27Befund allein keinesfalls die Diagnose sichert. In der Primärversorgung müssen die nicht geringen Testkosten von HLAB27 berücksichtigt werden, eine gesundheitsökonomische Analyse liegt hierzu allerdings bis jetzt nicht vor.

Eine Verlaufskontrolle des HLA-B27 ist nicht sinnvoll. Dies gilt sowohl für HLA-B27-positive als auch für -negative Befunde, da die kommerziellen Testverfahren nur wenig falsch negative Befunde produzieren (2-3\%).

Abhängig von der untersuchten Kohorte sind im Mittel 80-95\% der Patienten mit AS HLA-B27-positiv. Betrachtet man die Gesamtgruppe der Patienten mit axialer SpA inklusive der nr-axSpA, fällt dieser Durchschnittswert auf 60-85\% [74, 192]. HLA-B27-positive Patienten mit axialer SpA haben insgesamt einen schwereren Verlauf [16, 193-198]. Kohortenstudien haben gezeigt, dass die HLA-B27-positiven Patienten früher erkranken, eine höhere Krankheitsaktivität aufweisen, an größeren funktionellen Einbußen leiden und häufiger eine Uveitis und kardiale Manifestationen entwickeln, während HLAB27-negative Patienten später und meist weniger schwer erkranken, sie leiden aber häufiger an Psoriasis vulgaris und/ oder einer CED [193]. Bei Patienten mit einer kurzen Krankheitsdauer haben HLA-B27-positive Patienten häufiger als HLA-B27-negative Patienten eine in der MRT nachweisbare Entzündung in den SI-Gelenken (OR 2,13) [194]. HLA-B27 und männliches Geschlecht beeinflussen unabhängig voneinander die Wahrscheinlichkeit, eine kernspintomographisch nachweisbare Sakroiliitis aufzuweisen [195]. In dieser Studie ist bei HLA-B27-negativen Patienten ohne kernspintomographisch nachweisbare Sakroiliitis die Wahrscheinlichkeit, ein positives MRT nach 2 Jahren entwickelt zu haben, nur 5\%. Für die Schwere der Erkrankung scheint es unwichtig zu sein, ob HLA-B27 homozygot vorliegt [198]. Die Analyse von HLA-B27-Polymorphismen ist für die klinische Versorgung ebenfalls nicht relevant [199].

5.2.2.2 CRP und BSG. Die Bestimmung von CRP und/oder Blutsenkungsgeschwindigkeit (BSG) kann zur Abklärung der Verdachtsdiagnose axiale SpA eingesetzt werden, aber nur etwa die Hälfte der Patienten mit axialer SpA weist erhöhte Spiegel der Entzündungsparameter auf $[74,200]$. Die Post-TestWahrscheinlichkeit eines erhöhten CRP oder der BSG liegt nur bei $12 \%$. Die Sensitivität erhöhter Entzündungszeichen liegt zwischen 38 und 69\%, Spezifität bei $67-80 \%$ und die positive LR bei 2,5 [43]. Wegen der geringen Kosten wird die Bestimmung des CRP in der Primärversorgung aber häufig durchgeführt und kann bei erhöhten Werten differenzialdiagnostisch hilfreich sein, da Patienten mit nichtspezifischen Kreuz- 
schmerzen im Prinzip keine erhöhten Entzündungszeichen aufweisen.

Siehe auch ausführliche Darstellung von CRP und BSG in Abschn. 6.2.

\subsubsection{Variablen der bildgebenden Diagnostik}

Die Wahrscheinlichkeit, dass bei einem Patienten die Verdachtsdiagnose einer axialen SpA bestätigt wird, ist bei Vorliegen von strukturellen Läsionen im konventionellen Röntgenbild oder von kernspintomographisch darstellbaren Entzündungen im SI-Gelenk hoch (sie ist nicht $100 \%$, da auch Differenzialdiagnosen zu erwägen sind wie stärkere mechanische Belastung oder auch in seltenen Fällen mal infektiologische Ursachen). Der Vorteil der MRT-Diagnostik ist die hohe Sensitivität und Spezifität. In der Berliner Kohorte war die MRTUntersuchung die sensitivste Methode zur Diagnose einer axialen SpA $(69,7 \%)$ [191]. Hervorzuheben bleibt aber, dass in dieser Kohorte die Mehrzahl der überwiesenen Patienten eine radiologisch nachweisbare Sakroiliitis aufwiesen verglichen mit den (wenigen) Patienten, die aufgrund einer in der MRT darstellbaren Sakroiliitis überwiesen wurden. Wegen der für Patienten mit entzündlichen Rückenschmerzen oft nicht optimalen Initialbildgebung (z.B. aufgrund einer falschen Methodenwahl oder einer ungünstigen Auswahl des Bildbereiches) sollte die Indikation für Bildgebung bei Patienten in der Primärversorgung eher zurückhaltend gestellt werden.

Die Wertigkeit von MRT und Röntgen sind im Abschn. 6 Bildgebung ausführlich dargestellt.

\subsubsection{Kombination von Variablen}

Das Ziel, selektiv Patienten mit einer hohen Wahrscheinlichkeit für axiale SpA zur weiteren Abklärung bzw. Diagnosesicherung zum Rheumatologen zu überweisen, gelingt am besten mit einer Kombination von Variablen. Einzelvariablen können nur eingeschränkt verwendet werden, aber die Kombination von mehreren Variablen erhöht die prädiktive Aussagekraft. Alle oben genannten Strategien beruhen auf einer Kombination von Variablen [188, 190, 191, 201]. In einer niederländischen Kohorte wurde bei
364 Patienten mit chronischen Rückenschmerzen überprüft, welche Variablen der ASAS-Klassifikationskriterien bei Patienten mit Rückenschmerzen vorliegen [202]. Hierbei zeigte sich, dass die Variablen ERS, positive Familienanamnese, gutes Ansprechen auf eine NSARTherapie und Symptomdauer hinweisend für die Diagnose einer axSpA sein können. Allerdings wurde in dieser Studie das Erfüllen der ASAS-Klassifikationskriterien gleichgesetzt mit der Diagnosestellung einer axSpA - welches der Verwendung von Klassifikationskriterien zuwiderläuft (s. Abschn. 4). In einer US-amerikanischen Untersuchung zeigte sich, dass bei $63 \%$ der Risikopatienten (18 bis 44 Jahre alte Patienten mit Rückenschmerzen) in 101 zufällig ausgewählten rheumatologischen Praxen die ASAS-Klassifikationskriterien positiv waren, woraus sich eine nationale Prävalenz von 0,70\% (95\%-CI 0,38-1,1\%) ergibt [184].

Der Vorteil von Überweisungsstrategien, die auf einer Kombination aus Variablen bestehen, konnte in der internationalen RADAR-Studie gezeigt werden [203]. Wenn 3 Variablen (ERS, Sakroiliitis oder HLA-B27) kombiniert wurden, konnte die Diagnose axSpA bei einem Drittel der Patienten gestellt werden. Wenn mehr als 3 Variablen (z. B. Familienanamnese, gutes Ansprechen auf eine NSAR-Therapie, Vorhandensein von EAM) berücksichtigt werden konnten, wurde die Diagnose bei 35,6\% der Patienten gestellt. Innerhalb der SPACE Kohorte wurde evaluiert, welche Überweisungsstrategie eine hohe diagnostische Wertigkeit hat [204]. Es wurde geziegt, dass keine der Überweisungsstrategien auf internationaler Ebene uneingeschränkt empfohlen werden kann, da sie entweder zu zeitaufwendig oder zu kostenintensiv sind.

Aufgrund der hohen Prävalenz chronischer Rückenschmerzen in der Allgemeinbevölkerung ist die Frage, welche SpA-Variablen zur Selektion geeigneter Patienten in besonders hohem Maße beitragen, relevant. Auf die diagnostische Wertigkeit verschiedener Variablen wurde bereits im Abschn. 4.1 eingegangen. In der spanischen EPSERANZA-Kohorte wurde diese Frage mit Hinblick auf die eher teuren Untersuchungen der HLAB27-Bestimmung und der MRT-Untersuchung analysiert [32]. Die Kollegen konnten zeigen, dass das Vorhandensein eines alternierenden Gesäßschmerzes $(L R+2,6)$, einer Daktylitis $(L R+4,1)$ oder einer CED $(\mathrm{LR}+6,4)$ die Wahrscheinlichkeit, bei Patienten mit chronischen Rückenschmerzen eine Sakroiliitis in der MRT zu identifizieren, erhöht. Lagen eine Daktylitis oder eine CED vor, erhöhte sich die Wahrscheinlichkeit für eine Sakroiliitis in der MRT von 40 auf $79 \%$. HLA-B27 hatte in dieser Untersuchung keine hohe Vorhersagekraft.

Es fehlen randomisierte Studien, die zeigen, dass die Anwendung von Überweisungsstrategien zu einer Verkürzung der Diagnoseverzögerung oder einer Verhinderung der Zunahme röntgenologischer Progression führt.

\section{Empfehlung/Statement}

5-1

Die axiale SpA ist eine wichtige Differenzialdiagnose bei Patienten mit chronischen Rückenschmerzen, wenn diese vor dem 45. Lebensjahr beginnen. Empfehlungsgrad: Statement Evidenz: -

\section{5-2}

Patienten mit chronischen Rückenschmerzen ( $\geq 3$ Monate), einem Alter bei Beginn der Rückenschmerzen $<45$ Jahre und mindestens einem weiteren Parameter, der für eine SpA spricht, sollen zur weiteren Klärung zum Rheumatologen überwiesen werden. Besonders geeignete Parameter sind: - entzündlicher Rückenschmerz (s. Empfehlung 3-1), - Nachweis von HLA-B27.

\section{Empfehlungsgrad: NA \\ Evidenz: NA}

\section{Kommentar zu NA:}

Eine methodenkritische Bewertung ist bei diesen Studien überwiegend nicht möglich, da es sich weder um Kohorten noch um Fall-Kontroll-Studien im definierten Sinne handelt. Daher wurde weder ein Empfehlungsgrad noch ein Evidenzlevel vergeben. 


\section{Diagnostik}

\section{Schlüsselfrage 8}

- Wann und wie oft sollte welche Bildgebung/welches Labor bei Patienten mit SpA durchgeführt werden (Stellenwert)?

\subsection{Bildgebung}

Die Bildgebung ist bei Patienten mit axialer SpA essenziell für die Diagnose und das Management der Erkrankung - dies schließt die Bestimmung der Krankheitsaktivität (Entzündung) und die Messung von strukturellen Schäden (Knochenneubildung, Erosionen) mit ein. Die wichtigsten Lokalisationen von pathologischen Veränderungen bei axialer SpA sind die SI-Gelenke, die Wirbelsäule und die Hüftgelenke.

Grundsätzlich ist zwischen aktiven entzündlichen und chronischen strukturellen Veränderungen zu unterscheiden. Bei Patienten mit SpA kann ein Nebeneinander von entzündlichen Veränderungen (z.B. Nachweis eines Knochenmarködems bei Spondylitis) und strukturellen Prozessen der Knochenneubildung (z. B. Syndesmophyten, Ankylose) vorliegen. Inwieweit diese charakteristischen pathophysiologischen Phänomene zusammenhängen, ist nicht vollständig klar [205].

Es liegen verschiedene Studien über den Zusammenhang von Entzündung und Knochenneubildung in der Wirbelsäule bei AS-Patienten vor [205-209]. Einige Studien zeigen, dass ein Teil der Syndesmophyten an denjenigen Stellen entsteht, an denen meist 2 Jahre vorher eine Entzündung dargestellt werden konnte [207]. Auf der anderen Seite gab es parallel dazu auch Knochenneubildung ohne vorherigen Nachweis von entzündlicher Aktivität [205].

Die Rolle chronischer struktureller Veränderungen im Achsenskelett wie fettige Läsionen und die daraus resultierende Potenz zur Knochenneubildung wird zurzeit erforscht. Vor Kurzem veröffentlichte Studien haben gezeigt, dass sowohl ein initial erhöhtes CRP als auch das Vorhandensein von Syndesmophyten bei der Erstvorstellung von Patienten mit AS die Entwicklung neuer knöcherner Veränderungen im weiteren Verlauf vorhersagen können [210]. Eine wichtige pathophysiologische Hypothese ist, dass die Entzündung das Wachstum von Syndesmophyten begünstigt und dass das Wachstum von Syndesmophyten indirekt durch Unterdrückung der Entzündung oder direkt durch Unterdrückung der Knochenneubildung verhindert oder zumindest verlangsamt werden kann. Mögliche Gründe dafür sind, dass (i) entzündliche Aktivität und Knochenneubildung, zumindest zum Teil, voneinander unabhängig verlaufen („uncoupling“) und/oder (ii) eine Therapie mit TNFi durch Osteoklasteninhibition und Osteoblastenaktivierung über eine Modifikation des sog. Wnt und/oder RANKL-Systems den ankylosierenden Prozess bei AS-Patienten sogar fördern kann [211-213].

Um die richtige Methode der Bildgebung auswählen zu können, muss der Untersucher neben der Lokalisation zunächst festlegen, ob entzündliche Veränderungen oder ob morphologische strukturelle Veränderungen im Fokus der Fragestellung stehen (•Tab. 7). Die Fragestellung bestimmt somit die Auswahl des bildgebenden Verfahrens.

\subsubsection{Konventionelle Röntgentech- niken}

Bei der konventionellen Radiographie steht die Darstellung chronischer knöcherner Strukturveränderungen im Vordergrund. Diese treten bei Patienten mit axialer SpA vorwiegend in der Wirbelsäule sowie den SI- und Hüftgelenken als Folge vorausgegangener Entzündungszustände auf. Die Knochenneubildung am Achsenskelett mit Ausbildung von Syndesmophyten gilt als besonders charakteristisch für die AS [1, 210].

\subsubsection{Röntgenuntersuchung der Sa-} kroiliakalgelenke. Der entzündliche Krankheitsprozess bei axialen SpA beginnt in der Mehrzahl der Fälle in den SakroiliakaIgelenken (SI-Gelenke). Die Indikation für die röntgenologische Darstellung erfolgt in Abhängigkeit von der Lokalisation der angegebenen Beschwerden: Bei typischem tief sitzendem entzündlichem Rückenschmerz sollte eine Röntgen-Beckenübersichtsaufnahme anterior-posterior erfolgen. Diese ist ebenfalls sinnvoll bei Verdacht auf Beteiligung der Hüftgelenke. Bei parallelem Vorliegen von höher liegenden Rückenschmerzen ist eine Darstellung der LWS a.-p. (mit weiter Aufblendung zur Darstellung der Sakroiliakalgelenke) und lateral der konventionellen Beckenübersicht vorzuziehen. Die Spezialaufnahme der SI-Gelenke nach Barsony bzw. die Beckenübersichtsaufnahme nach Ferguson ermöglicht dagegen nur die Darstellung der SI-Gelenke ohne bzw. mit verzerrter Abbildung der Hüftgelenke. Die Sensitivität der Untersuchung wird bei Patienten mit AS mit 35\% angegeben, die Spezifität liegt zwischen 95 und $100 \%$ (abhängig von der untersuchten Population) [42].

Das Röntgenbild der SI-Gelenke ist für die qualitative Differenzierung zwischen AS (= M. Bechterew) und nr-axSpA definitionsgemäß ausschlaggebend. Hierbei wird der Schweregrad der strukturellen Veränderungen an den SI-Gelenken quantifiziert. Unter einer ankylosierenden Spondylitis (AS $=$ Morbus Bechterew) versteht man daher diejenige Form der axialen $\mathrm{SpA}$, die bereits röntgenologisch sichtbare knöcherne Veränderungen in den Sakroiliakalgelenken (SI-Gelenken) und/oder der Wirbelsäule hervorgerufen hat. Die Graduierung der Veränderungen an den SI-Gelenken ist die Grundlage der Klassifikation einer AS nach den 1984 modifizierten New-YorkKriterien (s. Abschn. 4.2) (• Tab. 8).

Zur Klassifikation (oft auch für Diagnosestellung verwendet) einer AS müssen nach diesen Kriterien neben einem von 3 klinischen Zeichen strukturelle Veränderungen (= röntgenologische Sakroiliitis) mindestens Grad 2 beidseits oder unilateral Grad 3 oder 4 vorliegen [175]. Eine Diagnosestellung nur auf der Basis von Wirbelsäulenveränderungen (meist Syndesmophyten bzw. Ankylose) ist möglich, aber vergleichsweise selten.

Häufig treten knöcherne Veränderungen erst relativ spät im Krankheitsprozess auf - zum Teil dauert es mehrere Jahre [214]. In sog. Frühkohorten finden sich aber auch Patienten, die schon nach einer kurzen Krankheitsdauer eine röntgenologische Progression in den SI-Gelenken 
Tab. 8 Scoring der SI-Gelenke, Graduierung nach den modifizierten New-York-Kriterien [175]

\begin{tabular}{ll} 
Graduierung & Veränderungen \\
\hline Grad 0 & Normal, keine Veränderungen \\
Grad I & $\begin{array}{l}\text { Verdächtige, mögliche Veränderungen } \\
\text { Grad II }\end{array}$ \\
$\begin{array}{l}\text { Minimale Veränderungen, minimale Sakroiliitis (geringe Sklerose, Erosionen, } \\
\text { keine Gelenkspaltveränderungen) }\end{array}$ \\
Grad III & $\begin{array}{l}\text { Eindeutige Veränderungen, moderate Sakroiliitis (deutliche Sklerose, Erosionen, } \\
\text { Gelenkspaltverbreiterung oder Gelenkspaltverschmälerung) }\end{array}$ \\
Grad IV & Ankylose
\end{tabular}

aufweisen. Die röntgenologische Progression war definiert mit „Verschlechterung von mindestens einem Grad gemäß der modifizierten New-York Kriterien“. In der niederländischen Kohorte $(n=68)$ hatten $20 \%$ der Patienten schon nach nur 18 Monaten röntgenologische Auffälligkeiten in den SI-Gelenken [180]. In einer deutschen Frühkohorte $(54,8 \%$ AS-Patienten, 45,2\% nr-axSpA-Patienten) mit einem Symptombeginn $<5$ Jahre trat nach 2 Jahren bei 10,5\% der Patienten mit nr-axSpA eine röntgenologische Progression in den SI-Gelenken auf [215]. In der französischen Frühkohorte lag die Progressionsrate an den SIG über 5 Jahre bei 5,1\% (Wechsel nr-axSpA zu AS) [216]. Epidemiologische Daten aus der Rochester-Kohorte zeigen, dass Patienten mit nr-axSpA über Jahrzehnte keinen Progress der Erkrankung aufweisen können (Wahrscheinlichkeit des Verbleibens als nr-axSpA in 15 Jahren: $73,6 \%$ [95\%-CI, 62,7-86,3]) [217]. Eine weitere Studie aus den USA zeigte eine ähnlich hohe Rate an Persistenz im nraxSpA-Klassifikationsarm [217]. Hierbei ist allerdings zu beachten, dass diese Patienten zwar die ASAS-Klassifikationskriterien für axSpA erfüllen, es bleibt aber unklar, ob die Diagnose einer axSpA auch rheumatologisch gestellt wurde.

\subsection{Differenzialdiagnose der Sa-} kroiliitis im Röntgenbild. Als Differenzialdiagnose der Sakroiliitis müssen im konventionellen Röntgenbild die entzündlichen Läsionen von degenerativen Läsionen, tumorösen oder septischen Läsionen abgegrenzt werden. Die Osteitis condensans ilii (= Hyperostosis triangularis) und Veränderungen im Rahmen eines DISH-Syndroms (diffuse idiopathische Skeletthyperostose, Morbus Forestier) (M48.19) müssen als be- sondere Formen degenerativer Veränderungen im SI-Gelenk von der Sakroiliitis im Rahmen einer axialen SpA abgegrenzt werden. Erkrankungen aus dem rheumatischen Formenkreis wie SAPHOSyndrom (Synovitis, Akne, Pustulosis, Hyperostosis, Osteitis) (M86.3), Morbus Behçet (M35.2), Morbus Paget (M88.99) sowie Psoriasisarthritis (L40.5, ${ }^{\star}$ M07.3) können ebenfalls das Bild einer Sakroiliitis hervorrufen. Eine rein morphologische Differenzierung ohne Berücksichtigung der klinischen Symptome (aktuell und auch anamnestisch) ist anhand der Röntgenbilder oft nicht möglich.

Die früher häufiger durchgeführten röntgenologischen Schichtuntersuchungen der SI-Gelenke (Tomographie) gelten heute wegen der Strahlenexposition und der Verfügbarkeit von MRT als obsolet.

\subsubsection{Röntgenuntersuchung der Wir-} belsäule. Bei mehr als der Hälfte der Patienten mit AS manifestiert sich der Krankheitsprozess im Verlauf der Erkrankung auch an der Wirbelsäule. Die Wirbelsäule kann in allen Etagen betroffen sein, am häufigsten ist jedoch der thorakolumbale Übergang beteiligt [218]. Syndesmophyten und Ankylosierungen sind pathognomonisch für die AS. Weitere typische Röntgenveränderungen in der Wirbelsäule von AS-Patienten sind:

- Sklerosierungszonen an den Wirbelkörperecken (glänzende Ecken $=$, shiny corners" $=$ RomanusLäsion), wahrscheinlich als Folge einer stattgehabten Spondylitis,

- Erosionen in den Grund- oder Deckplatten der Wirbelkörper (AndersonLäsion), wahrscheinlich als Folge einer stattgehabten Spondylodiszitis, - Bildung von sog. Kasten- oder Tonnenwirbeln, wahrscheinlich als Ausdruck von entzündlichen
Veränderungen an den Wirbelkörperkanten,

- Wirbelfrakturen, die vor allem im späteren Krankheitsverlauf auftreten.

Sind bei einem Patienten Strukturveränderungen eingetreten, liegt meist auch eine Beeinträchtigung der körperlichen Funktionsfähigkeit und Lebensqualität vor [219]. Der Schweregrad der röntgenologischen Progression (Knochenneubildung) bei Patienten mit axialer SpA ist variabel [220]. Zirka ein Viertel der Patienten mit AS hat einen schnellen Progress [20] (s. Abschn. 7). Bei Patienten der deutschen Inzeptionskohorte GESPIC (AS und nr-axSpA) lag die Progressionsrate an der Wirbelsäule (Differenz von $\geq 2$ mSASSS-Punkten) bei $15,2 \%$ in einem Beobachtungszeitraum von 2 Jahren. Dieselben Autoren konnten zeigen, dass $11,2 \%$ der Patienten neue Syndesmophyten entwickelten oder an existierenden Syndesmophyten einen Progress zeigten [221]. In der niederländischen Kohorte mit ausschließlich ASPatienten lag die Rate neuer Syndesmophyten bei $33 \%$ in 2 Jahren [222]. In allen Kohorten konnte gezeigt werden, dass eine hohe Krankheitsaktivität mit einem hohen Risiko der röntgenologischen Progression assoziiert ist [223]. In der deutschen Kohorte lagen folgende Prädiktoren für eine Progression vor: bei Baseline vorhandene Syndesmophyten (OR 6,29), erhöhter CRP-Wert (OR 3,8) und Nikotinkonsum (OR 2,75) [224]. In einer niederländischen Kohorte lag das relative Risiko, innerhalb von 4 Jahren neue Syndesmophyten zu entwickeln, bei 5,0 (95\%-CI 2,5-10,2) [222].

Auf den möglichen Einfluss von NSAR und Biologika auf die röntgenologische Progression wird in Abschn. 8.1.4 (NSAR) und 8.4.2 (Biologika) noch näher eingegangen.

Die LWS und Brustwirbelsäule (BWS) werden in der Regel in 2 Ebenen geröntgt (a.-p. und lateral), wohingegen zur Beurteilung von strukturellen Läsionen in der HWS oft eine seitliche Aufnahme ausreicht. Die Indikation, welcher Abschnitt der Wirbelsäule geröntgt wird, ergibt sich aus der klinischen Beurteilung. Hierbei ist $\mathrm{zu}$ beachten, dass bei einigen Patienten nicht ausschließlich der Schmerz 
im Vordergrund stehen muss, sondern der behandelnde Arzt kann z. B. bei einer Zunahme der Funktionseinschränkung auch ohne begleitende Schmerzsymptomatik eine Röntgenuntersuchung veranlassen. Eine Röntgenuntersuchung der Wirbelsäule ist in der Regel nicht häufiger als alle 2 Jahre indiziert [225]. Dies liegt zum einen an dem im Mittel geringen Progress bei den AS-Patienten und der begrenzten Sensitivität für Veränderungen der Scoring-Methoden sowie der kumulativen Strahlenexposition bei diesen zum Teil relativ jungen Patienten.

Für die Quantifizierung struktureller Veränderungen der Wirbelsäule sind 3 verschiedene Scores entwickelt worden, die aber lediglich im wissenschaftlichen Kontext ihre Berechtigung haben und deshalb hier nicht einzeln aufgeführt werden [226-229].

\subsection{Differenzialdiagnose der Wir- belsäulenveränderung im Röntgen-} bild. Als Differenzialdiagnose müssen Spondylophyten als morphologisch führendes Bild degenerativer Veränderungen von den Knochenanbauten im Rahmen einer SpA abgegrenzt werden. In einer niederländischen Untersuchung zeigte sich innerhalb der SAPCE-Kohorte (junge Patienten mit chronischen Rückenschmerzen hinweisend auf eine SpA) eine hohe Prävalenz an degenerativen Wirbelsäulenveränderungen (zwischen 50 und $90 \%$ für verschiedene Degenerationen) [230]. Spondylophytäre Veränderungen können im Rahmen eines DISH-Syndroms bestehen, wobei multiple benachbarte Wirbelsegmente durch überschießende ventrale Ossifikationen überbrückt sind, häufig an der unteren HWS und der gesamten BWS. Erkrankungen aus dem rheumatischen Formenkreis wie SAPHO-Syndrom, Morbus Paget sowie Psoriasisarthritis sind in der differenzialdiagnostischen Abklärung von Knochenneubildungen der Wirbelsäule zu bedenken.

\subsubsection{Erfassung der Knochendichte} und Erfassung von Wirbelkörperdeformitäten. Die Prävalenz von Frakturen der Wirbelsäule ist bei Patienten mit AS erhöht (s. Abschn. 3.1.1.4) [48-50, 52]. Die Erfassung von stärkeren Schmerzen in der Wirbelsäule und von plötzlichen Veränderungen im Krankheitsverlauf ist daher wichtig und sollte Anlass für eine Abklärung geben. Zum Nachweis von Wirbelkörperdeformitäten sind verschiedene Verfahren entwickelt worden, wobei sich die Technik der Bildgebung und die Definition „Fraktur" unterscheiden. Zwei Methoden sind bei postmenopausalen Frauen evaluiert worden: die radiologische Wirbelkörpermorphometrie (MRX) und eine Wirbelkörpermorphometrie auf der Basis einer DXAMessung (MXA). Beide Verfahren sind in einer Studie an 30 AS-Patienten getestet worden [231]. Die Ergebnisse beider Methoden sind auf einem globalen Level vergleichbar, nicht jedoch, wenn einzelne Wirbelkörper miteinander verglichen werden.

Die Messung der Knochendichte kann durch das Vorhandensein von Syndesmophyten verfälscht sein, weshalb die Messwerte der LWS mit Vorsicht interpretiert werden müssen. Schwedische Kohortendaten zeigen, dass sich die Knochendichte bei AS-Patienten zwischen Hüfte und LWS unterscheidet und an der Hüfte deutlich niedrigere Messwerte vorliegen [51].

\subsubsection{MRT}

Die Magnetresonanztomographie(MRT) dient vor allem der Diagnostik früher und aktiver Entzündungsstadien. Die Entzündung kann in der Wirbelsäule, den SI-Gelenken, peripheren Gelenken oder extraartikulären Strukturen wie Sehnenansätzen (Enthesen) dargestellt werden. Die MRT-Technik erlaubt auch die Erfassung von bestimmten strukturellen Veränderungen wie lokalen Verfettungen; Knochenneubildungen oder Erosionen sind ebenfalls sichtbar. Die Erfassung struktureller Veränderungen in der MRT ist mit den derzeit verbreiteten Standardverfahren jedoch deutlich schlechter standardisiert. Neuere Untersuchungen zeigen, dass im Vergleich zur CT-Untersuchung (Goldstandard in der Erfassung struktureller Läsionen) mit der MRT mehr und zuverlässiger Erosionen in den SIG erfasst werden können als in der konventionellen Röntgenuntersuchung [232].
Es gibt insgesamt 4 MRT-Techniken, die sowohl in der klinischen Praxis als auch bei klinischen Studien eingesetzt werden. Grundsätzlich muss bei pathologischen Befunden zwischen entzündlichen und strukturellen bzw. chronischen Veränderungen unterschieden werden. T1-gewichtete Spinechosequenzen werden eingesetzt, um strukturelle Veränderungen zu erfassen. Dabei zeigt sich das Fettmark als hyperintense Struktur, wodurch gut zwischen verschiedenen anatomischen Strukturen differenziert werden kann. Entzündliche Veränderungen können entweder mit T2-gewichteten fettsupprimierten Sequenzen (T2/FSE), mit ,short-tau inversion recovery" (STIR) oder mit T1-gewichteten fettsupprimierten Sequenzen nach Gabe von Kontrastmittel (normalerweise Gadolinium (auch Gadopentetat-Dimeglumin $[\mathrm{T} 1 / \mathrm{Gd}]$ ) genannt) dargestellt werden. Diese letzten 3 MRT-Techniken zeigen die entzündlichen Läsionen als hyperintense Strukturen, wobei sich normales Knochenmark als hypointense Struktur darstellt. Die T2/FS- und die STIR-Sequenz sind sensitiv für die Erfassung von Flüssigkeit, sodass auch pathologische Flüssigkeit, wie z. B. beim Knochenmarködem, als hyperintenses Signal dargestellt wird. Die T1/Gd-Sequenz zeigt entzündliche Läsionen als hyperintenses Signal. Hierbei führt die vermehrte Vaskularisierung im Falle von Entzündung zu einer Diffusion von Kontrastmolekülen in das Interstitium. Es kann keine Präferenz für oder gegen STIR und T1-Sequenz mit Kontrastmittel gegeben werden, da beide Sequenzen entzündliche Areale adäquat abbilden [233, 234]. Die Kontrastmittelgabe ist aber teurer und hat keinen eindeutigen Vorteil gegenüber der STIR-Technik [233].

Ein in der klinischen Praxis häufig benutztes MRT-Protokoll beinhaltet eine sagittale T1- sowie eine sagittale STIRSequenz mit einer Bildmatrix von $512 \mathrm{Pi}$ xel und einer Schichtdicke von 3-4 mm. Üblicherweise werden 1,5-T-MRT-Geräte für die Untersuchung dieser Patienten benutzt, neuere Geräte mit höheren Feldstärken sind ebenfalls einsetzbar.

Die (experimentelle) GanzkörperMRT wird bei Patienten mit AS/SpA 
eingesetzt, um verschiedene Pathologien (Spondylitis, Enthesitis, Arthritis) multilokulär zeitgleich abzubilden. Somit können die gesamte WS inklusive SIGelenken, die Hüft- und Schulterregion sowie die vordere Thoraxapertur untersucht werden $[235,236]$. Für die SIGelenke und die Wirbelsäule liegt eine sehr gute Korrelation zwischen Ganzkörper-MRT und konventioneller MRTUntersuchung vor [237, 238].

\subsubsection{MRT der Sakroiliakalgelenke.}

Für die SI-Gelenke wird eine Schnittführung in schräg koronarer Schicht (parallel zu einer Linie, die den oberen dorsalen Anteil von S1 und S3 tangiert) empfohlen. Die Erfassung von pathologischen MRT-Veränderungen in den SI-Gelenken ist bei Patienten mit früher SpA von großer Bedeutung, da bis zur Ausbildung von strukturellen, im konventionellen Röntgenbild sichtbaren Veränderungen zum Teil Jahre vergehen können, entzündliche Veränderungen in den SI-Gelenken jedoch schon frühzeitig darstellbar sind. Die durch MRT erfassten entzündlichen Veränderungen korrelierten in einer Untersuchung aus Deutschland gut mit dem histologischen Nachweis von entzündlichen Zellinfiltraten [239]. In einer aktuellen Studie aus China wurde die gute Korrelation aber wieder infrage gestellt. Hier lag die Sensitivität nur bei 37,7\% [240].

Entzündliche Aktivität in den SI-Gelenken findet man bei MRT-Untersuchungen von Patienten mit Sakroiliitis auf dem Boden einer SpA in allen Stadien unabhängig von der Krankheitsdauer und vom Ausmaß der bereits stattgehabten strukturellen Veränderungen [241]. Es gibt verschiedene Vorschläge, wie MRTs der SI-Gelenke systematisch ausgewertet und die Veränderungen quantitativ erfasst werden können. Alle Methoden sind nicht validiert, und es gibt keine vergleichenden Studien [242-245]. Im Wesentlichen erfolgt die Graduierung aktiv entzündlicher und chronisch struktureller Läsionen bezogen auf 4 Quadranten pro SI-Gelenk.

Die ASAS/OMERACT-Gruppe MRT hat als Zeichen einer aktiven Entzündung in den SI-Gelenken folgende Pathologika definiert [234]:
- Knochenmarködem: Typischerweise liegt das Knochenmarködem periartikulär und subchondral. Zur Bewertung einer aktiven Entzündung sollte es obligat vorhanden sein.

- Synovitis, Enthesitis oder Kapsulitis: Wenn diese Veränderungen alleine ohne subchondrales Knochenmarködem bestehen, ist dies vereinbar mit, aber nicht ausreichend für die Diagnosestellung einer aktiven Sakroiliitis.

Als Zeichen chronischer Veränderungen in den SI-Gelenken gelten:

- Erosionen: Diese befinden sich an der Knorpel-Knochen-Grenze und stellen ein wichtiges Merkmal zur Diagnose der SpA dar.

- Subchondrale Sklerose: Sie kann sowohl bei Patienten mit SpA als auch bei Gesunden vorkommen.

- Fettmetaplasie: Sie gilt als Zeichen einer abgelaufenen Entzündung.

- Ankylose oder Knochenbrücken: Sie können so stark ausgebildet sein, dass das SIG komplett durchbaut ist.

Zurzeit wird diskutiert, ob chronische Veränderungen in der MRT-Untersuchung allein oder in Kombination mit akut-entzündlichen Veränderungen für die Diagnose einer Sakroiliitis als Hinweis auf eine SpA mit verwendet werden sollten. Erosionen und Fettmetaplasie scheinen in der Beurteilung bei Patienten mit SpA eine wichtige Rolle zu spielen. Fettmetaplasie im SIG ist ein spezifisches Zeichen für axSpA, jedoch nur wenig änderungssensitiv [246]. Die diagnostische Aussagekraft von Erosionen ist aber nicht vollständig klar, insbesondere wenn es sich um geringe Veränderungen und um einzelne Läsionen handelt [247]. Eine aktuelle Fall-Kontroll-Studie kommt zu dem Schluss, dass die Kombination Sakroiliitis plus Erosion die Sensitivität erhöht ohne Reduktion der Spezifität [248]. In einer kürzlich publizierten Studie aus der Charité Berlin konnte gezeigt werden, dass die MRT der SIG dem Röntgen im Vergleich zur CT in der Detektion struktureller Veränderungen am SI-Gelenk überlegen ist [232].
6.1.2.1.1 Diagnostischer Nutzen von MRT der SI-Gelenke. Der diagnostische Nutzen einer MRT-Untersuchung der SI-Gelenke ist sehr hoch. Allerdings ist die Angabe von Sensitivität und Spezifität sowie positiver und negativer LR problematisch, da ein Goldstandard, gegen den die MRT getestet werden kann, fehlt. In einer kanadischen Studie wurde der diagnostische Nutzen einer MRT der SI-Gelenke bei 187 geschlechts- und altersgematchten Gruppen untersucht [249]. Die Spezifität der MRT der SIGelenke bei Patienten mit gesicherter AS lag zwischen 94 und $99 \%$ und die Sensitivität zwischen 83 und $99 \%$. Die positive und negative LR lag bei 44,6 bzw. 0,10. Die Spezifität der MRT der SI-Gelenke bei Patienten mit entzündlichem Rückenschmerz schwankte zwischen 94 und $99 \%$, die Sensitivität lag niedriger bei $48-52 \%$. Die positive und negative LR lag bei 46,0 bzw. 0,50. Die Autoren fanden, dass die zusätzliche Beurteilung und Miteinbeziehung von Erosionen in den Gesamtscore zusätzlich zum alleinigen Knochenmarködem die diagnostische Aussage erhöhen können. Durch Hinzunahme der Erosionen konnten sie die Sensitivität von 67 auf $81 \%$ steigern bei gleichbleibender hoher Spezifität (von $88 \%$ ). Zusätzlich erhöhte das Nebeneinander von akuten entzündlichen Veränderungen und bereits strukturellen Veränderungen als Folge früherer Entzündungen die diagnostische Sicherheit der MRT der SI-Gelenke [250].

6.1.2.1.2 Indikation zur MRT der SI-Gelenke. Erstdiagnostik: Bei einem Verdacht auf eine axiale SpA und unauffälligem Röntgenbild der SI-Gelenke sollte zunächst eine MRT-Untersuchung der SI-Gelenke mit Entzündungssequenz durchgeführt werden, unabhängig von der Lokalisation des Rückenschmerzes. In den Studien wird über eine Häufigkeit eines positiven MRT-Befundes bei Patienten mit einem Verdacht auf SpA zwischen 26 und $85 \%$ berichtet [2, 188]. Es liegt keine signifikante Korrelation zwischen dem Ausmaß der akuten Entzündung und klinischen $\mathrm{Pa}$ rametern vor. Das Vorhandensein eines Knochenmarködems korrelierte nicht 
mit der Einnahme von NSAR [244]. Das Ausmaß der Entzündung in den SI-Gelenken ist bei HLA-B27-positiven Patienten größer [244, 251]. In einer britischen Studie konnte gezeigt werden, dass bei Patienten mit entzündlichem Rückenschmerz die Kombination von HLA-B27 mit einer ausgeprägten Sakroiliitis eine hohe Spezifität für die Entwicklung einer AS aufwies (positive LR 8,0; Spezifität $92 \%$ ) [252]. Bei Patienten mit gering ausgeprägter Sakroiliitis lag unabhängig vom HLA-B27-Status eine geringe Wahrscheinlichkeit vor, eine AS zu entwickeln.

Im Verlauf: In der Literatur werden nur wenige Studien mit Verlaufsuntersuchungen der MRT der SI-Gelenke sowohl bei axialer SpA als auch bei AS-Patienten vorgestellt [244, 245, 251]. Es kann keine sichere Aussage darüber getroffen werden, wie sich in der MRT nachweisbare Veränderungen in den SI-Gelenken im zeitlichen Verlauf verhalten. In einer dänischen Beobachtungsstudie bei Patienten mit axialer SpA, die die ESSGKriterien erfüllten, nahmen die Veränderungen, insbesondere der chronischen SIG-Veränderungen, über den beobachteten Zeitraum (im Mittel Folgeuntersuchung nach 51 Monaten) zu; allerdings wurde keine Aussage über mögliche Begleittherapien gemacht [251]. Innerhalb des 2-jährigen Beobachtungszeitraumes entwickelten $61 \%$ der Patienten eine AS. Diese Progressionsrate ist als hoch bzw. erhöht einzuschätzen. In einer britischen Beobachtungsstudie zeigte sich bei Patienten mit entzündlichen Rückenschmerzen ( $90 \%$ erfüllten ESSG-Kriterien) eine geringe Abnahme des Knochenmarködems über den beobachteten Zeitraum von 12 Monaten. Die Patienten hatten in der Mehrzahl eine NSAR-Therapie erhalten, $20 \%$ der Patienten waren neu aufSulfasalazin eingestellt worden [244]. In beiden Studien waren das Auftreten und das Ausmaß der Entzündung bei HLA-B27positiven Patienten größer. In einer neueren Studie aus Dänemark zeigte sich, dass sich unter einer 12-wöchigen Therapie mit Adalimumab das Ausmaß der Sakroiliitis stärker im Vergleich zur Plazebomedikation zurückbildete (Verum: Berlin Score: $-62 \%$, SPARCC Score: $-58 \%$, Pla- zebo: Berlin Score: $-5 \%$, SPARCC Score: $-12 \%)$ [253].

\subsection{Differenzialdiagnose der Sa-} kroiliitis in der MRT. In die Betrachtung müssen septische Sakroiliitiden, (Insuffizienz-)Frakturen, Knochentumoren oder eher degenerative Veränderungen wie bei Osteitis condensans ilii (Hyperostosis triangularis) mit einfließen. SpA-typische Veränderungen finden sich in der Regel innerhalb der anatomischen Grenzen und sind auf den Knochen und die SI-Gelenke limitiert.

In den Studien, die Aussagen zu Sensitivität und Spezifität der MRT bei SpA machen, ist bei ansonsten fehlendem „Goldstandard“ als Vergleichsmethode die Bestätigung der Diagnose durch einen Rheumatologen als Goldstandard mit den MRT-Ergebnissen verglichen worden. Daher wird die Evidenz nach den Oxford-Kriterien mit 1B bewertet, sofern die anderen Studiencharakteristika auf eine gute Methodik hinweisen.

6.1.2.2 MRT der Wirbelsäule. Die MRTUntersuchung der Wirbelsäule erfasst unterschiedliche Pathologien in verschiedenen bei AS betroffenen Wirbelsäulenstrukturen wie den Wirbelkörpern, den Bandscheiben und den Ligamenten und Sehnenansätzen in axialen, sagittalen und koronaren Schnittbildern. Dabei werden in der täglichen Praxis die sagittalen Schnittbilder als wichtig angesehen. Wie auch in den SI-Gelenken wird bei der Erfassung pathologischer Veränderungen in der Wirbelsäule bei Patienten mit SpA zwischen entzündlichen und strukturellen/chronischen Veränderungen unterschieden. Die Veränderungen treten in allen Regionen der Wirbelsäule auf; die untere BWS ist jedoch bevorzugt sowohl von entzündlichen (45-75\%) als auch von chronischen Veränderungen (60\%) betroffen [254, 255].

Die in der Wirbelsäule am häufigsten vorkommenden pathologischen Veränderungen sind die Spondylitis anterior und posterior, die (abakterielle) Spondylodiszitis (Andersson-Läsion) und die Arthritis/Enthesitis der Kostovertebral- und Kostotransversalgelenke sowie der Zygoapophysealgelenke [255]. Ei- ne pathologische Signalanhebung (als Zeichen entzündlicher Veränderung) in den STIR- oder T1/Gd-Sequenzen, interpretiert als Knochenmarködem oder Osteitis, entspricht in histopathologisch untersuchten Zygoapophysealgelenken von AS-Patienten dem Befund entzündlicher Zellinfiltrate [256]. Allerdings zeigte diese Studie auch, dass die MRT nicht immer sensitiv genug ist, Bereiche mit geringer Entzündungsaktivität in der Wirbelsäule sichtbar zu machen.

Entzündliche Veränderungen finden sich besonders häufig an den Wirbelkörperecken - die sog. Spondylitis anterior und posterior. Solche entzündliche Läsionen repräsentieren das Vorliegen eines Knochenmarködems bzw. einer Osteitis, sie können als Zeichen einer Enthesitis angesehen werden [257]. Nachdem in der Vergangenheit überwiegend die anterioren Anteile der Wirbelkörperecken im Fokus standen, zeigen neuere Studien, dass die posterioren Anteile genauso häufig betroffen sind [258, 259]. Wenn sich die entzündlichen Areale im posterioren Anteil der Wirbelsäule (also Facettengelenke und Kostovertebralgelenke) befinden, ist die diagnostische Aussagekraft hoch (LR 14,5) [259].

Entzündliche Veränderungen, welche die Wirbelkörperkanten und die Bandscheiben betreffen, werden (abakterielle) Spondylodiszitis oder Andersson-Läsion genannt [260]. Solche Veränderungen kommen zwar bei weniger als $10 \%$ der Patienten mit AS vor, bieten jedoch differenzialdiagnostische Schwierigkeiten [261]. In der STIR-Sequenz zeigen sie sich als hyperintenses Signal in der Bandscheibe und in einer oder beiden angrenzenden Wirbelkörper. In der Differenzialdiagnose muss auch an bakterielle Ursachen, wie z. B. Tuberkulose, oder an mechanische Ursachen wie die erosive Osteochondrosis intervertebralis gedacht werden.

Der Stellenwert chronischer Veränderungen in der Wirbelsäule wie Erosionen, Fettablagerungen und Syndesmophyten oder Ankylose ist bei Patienten mit axialer SpA nicht gut untersucht. Es gibt lediglich eine (retrospektive) Studie, die Aussagen zum diagnostischen Nutzen chronischer Veränderungen in der WS macht [262]. Es wiesen $31 \%$ der Pa- 
tienten mit früher SpA in dieser Kohorte chronische Veränderungen im Sinne einer Romanus-Läsion mit fettiger Degeneration auf. Die Mehrheit der Läsionen war in der BWS zu finden (60\%). In der Erfassung chronischer Veränderungen ist die MRT den konventionellen Röntgenbildern nicht überlegen [263].

Für die Erfassung entzündlicher Veränderungen der Wirbelsäule sind 3 verschiedene Scores entwickelt worden [264-267]. Da die Erfassung chronischer Läsionen schwieriger ist, gibt es lediglich Vorschläge für eine Quantifizierung [263]. Alle 3 Methoden zur Analyse von entzündlichen Veränderungen sind sowohl als zuverlässig und übertragbar als auch als sensitiv gegenüber Veränderung unter Therapie geprüft worden [268]. Auf Grundlage der Daten kann keine Methode priorisiert werden. Da die Scores im klinischen Alltag keine Rolle spielen, werden sie hier nicht gesondert aufgeführt.

Die Korrelation zwischen dem Ausmaß entzündlicher Läsionen in der MRT und der klinischen Krankheitsaktivität ist relativ niedrig [75, 269, 270]. Daher sollen häufige Wiederholungen von MRT-Untersuchungen vermieden werden - das gilt vor allem, wenn bereits negative $\mathrm{Be}$ funde vorliegen.

Es gibt nur wenig Übereinstimmung zwischen dem Ausmaß chronischer Veränderungen in der MRT und dem Ausmaß struktureller Veränderungen im konventionellen Röntgenbild [263, 271]. Dies beruht hauptsächlich auf der Tatsache, dass Syndesmophyten in der MRT nur schwer zu erkennen sind und andererseits in der MRT sichtbare Fettablagerungen nicht im konventionellen Röntgenbild dargestellt werden.

\subsection{Diagnostischer Nutzen der} MRT der Wirbelsäule. Für den Nachweis entzündlicher Areale in einer MRT der Wirbelsäule ist ein zusätzlicher diagnostischer Nutzen anzunehmen. Je mehr entzündete Areale in der Wirbelsäule vorliegen, umso höher ist die diagnostische Aussagekraft. In einer prospektiven Studie, die nach Geschlecht und Alter gematcht war, zeigten sich eine Sensitivität der MRT der Wirbelsäule bei ASPatienten von $69 \%$ und eine Spezifität von $94 \%$ mit einer positiven LR von 12,0 [235]. In der Gruppe der Patienten mit entzündlichem Rückenschmerz lagen die Sensitivität bei $32 \%$, die Spezifität bei $96 \%$ und die positive LR bei 8,0 . In dieser Studie fand sich bei $26 \%$ (9/35) gesunden Individuen mindestens eine entzündliche Läsion, allerdings bei nur $5 \%(2 / 35)$ mehr als 2 entzündliche Läsionen. Zeigten die entzündlichen Areale noch ein besonders intensives hyperintenses Signal und handelte es sich um jüngere Patienten, sind diese Befunde wegweisend für die Diagnose einer axialen SpA [252].

Der diagnostische Nutzen chronischer Veränderungen ist im Vergleich zum diagnostischen Nutzen entzündlicher Areale geringer. Die diagnostische Aussagekraft von Fettläsionen an der Vorderkante der Wirbelkörper wird mit einer positiven LR von 4,7 angegeben [262].

Der diagnostische Nutzen einer MRT der Wirbelsäule in Kombination mit einer MRT der SIG im Rahmen einer Diagnosesicherung bei nr-axSpA-Patienten wird als gering angesehen [272].

Die ASAS/OMERACT MRT-Gruppe hat als Zeichen einer aktiven Entzündung in den SI-Gelenken folgende Pathologika definiert [273]:

- Veränderungen, hinweisend auf entzündliche Läsionen:

- anteriore/posteriore Spondylitis,

- Spondylodiszitis,

- Arthritis der Kostovertebralgelenke,

- Arthritis der zygoapophysealen Gelenke,

- Enthesitis der Bandstrukturen der Wirbelsäule;

- Veränderungen, hinweisend auf strukturelle Läsionen:

- fettige Degeneration,

- Erosionen,

- Syndesmophyten,

- Ankylose.

Gemäß diesem Konsensuspapier gelten die anteriore/posteriore Spondylitis und die fettige Degeneration als besonders charakteristische Veränderungen. Die Definition einer „positiven MRT“, welche hinweisend auf eine spinale Manifestation der SpA ist, ist erfüllt, wenn an mehr als 3 Lokalisationen eine anteriore/ posteriore Spondylitis vorliegt.

6.1.2.2.2 Indikation der MRT der Wirbelsäule. Bei Patienten mit Verdacht auf eine Wirbelsäulenbeteiligung im Rahmen der axialen SpA sollte eine MRTUntersuchung der Wirbelsäule mit Entzündungssequenz durchgeführt werden. Die MRT der Wirbelsäule ist die zentrale Untersuchungsmethode für die Feststellung der Lokalisation und des Ausmaßes entzündlicher Veränderungen, insbesondere im Bereich der Wirbelkörper, aber auch der angrenzenden Strukturen.

Neben der Möglichkeit der Lokalisation entzündlicher Areale, kann das Ausmaß der Entzündung zur Vorhersage eines therapeutischen Ansprechens genutzt werden. Besteht eine ausgedehnte Entzündung in der Wirbelsäule, spricht dies für eine gute Ansprechwahrscheinlichkeit auf eine TNFi-Therapie [274]. Das Ausmaßder Entzündung (auch CRP) und die (Kürze der) Krankheitsdauer waren statistisch signifikante Prädiktoren für ein mindestens 50\%iges Ansprechen auf eine TNFi-Therapie in Woche $12 \mathrm{der}$ Behandlung. Unter einer TNFi-Therapie kann schon nach 6 Wochen eine Verbesserung der Wirbelsäulenentzündung nachgewiesen werden [275-278]. Es hat sich jedoch gezeigt, dass entzündliche Veränderungen in der Wirbelsäule trotz TNFi-Therapie oft nicht vollständig verschwinden. Bei ca. 30-40 \% der Patienten mit AS verbleiben entzündliche Restzustände [278]. In der klinischen Routine ist eine Kontrolle der entzündeten Areale mittels MRT wegen fehlender Konsequenzen zum jetzigen Zeitpunkt als nicht sinnvoll zu erachten.

Ein positiver Einfluss einer NSARTherapie auf die entzündlichen Läsionen in der WS ist bisher nicht nachgewiesen. Es gibt eine kleine offene Studie über 6 Wochen mit Etoricoxib, welche eine MRT-Messung der Wirbelsäule in Woche 0 und 6 umfasst (allerdings ohne Kontrollgruppe) [279]. Innerhalb dieser kurzen Zeit hatten sich keine Veränderungen in den entzündlichen Arealen der Wirbelsäule dargestellt. Diese Patienten waren jedoch schon klinisch NSAR-Versager bei Studieneinschluss. 
Bei Patienten mit Verdacht auf eine Wirbelfraktur sollte unverzüglich eine entsprechende Bildgebung veranlasst werden. Diese kann neben einer MRT-Untersuchung der Wirbelsäule auch eine Röntgendarstellung bzw. Computertomographie des entsprechenden Wirbelsäulenabschnittes umfassen. Die entsprechenden Details werden in Abschn. 3.1.1.4 und 8.6.3 dargestellt.

6.1.2.2.3 Differenzialdiagnose. $\mathrm{Zu}$ den wichtigsten Differenzialdiagnosen pathologischer MRT-Befunde gehören im Bereich der Wirbelsäule physiologisch vorkommende kleine dorsoventral verlaufende Gefäße und Hämangiome. Blutgefäße können vor allem dorsalseitig der Wirbelkörper gefunden werden und ein hyperintenses Signal in STIR oder T1/Gd aufweisen. Hämangiome unterscheiden sich von entzündlichen Läsionen hauptsächlich durch ihre Form und Lage (rund, gut abgegrenzt, in der Mitte des Wirbelkörpers).

Insgesamt gibt es wenige Daten zur Spezifität unterschiedlicher MRT-Läsionen im Rahmen der Differenzialdiagnose zwischen SpA und anderen Erkrankungen des Achsenskeletts. Im Bereich der Wirbelsäule können die im Röntgenbild als Andersson-Läsionen bezeichneten Auffälligkeiten im Frühstadium leicht mit Modic-I-Läsionen verwechselt werden. In ähnlicher Weise können ältere Andersson-Läsionen mit Modic-II-Läsionen verwechselt werden. Weiterhin gibt es bisher keine systematischen Untersuchungen über die Differenzierung zwischen aktivierter Facettengelenkarthrose bei degenerativen Wirbelsäulenbeschwerden und dem entzündlichen Befall dieser Gelenke bei Patienten mit axialer SpA. Das bedeutet, dass eine rein morphologische Differenzierung zwischen unterschiedlichen Diagnosen ohne Berücksichtigung der klinischen Symptome (aktuell und auch anamnestisch) anhand der MRT-Befunde allein oft nicht möglich ist.

\subsubsection{Sonographie}

Die Sonographie erlaubt die bildgebende Diagnostik entzündlicher Veränderungen am peripheren Skelettsystem und kann mit akzeptabler Sensitivität sowohl eine Arthritis als auch eine Enthesitis nachweisen. Allerdings ist die diskriminative Fähigkeit des Ultraschalls gerade bei Fersenschmerzen gering, da häufig positive Befunde auch bei Patienten ohne SpA vorkommen [280].

Zur Diagnostik der Arthritis sind viele kontrollierte Studien bei Patienten mit RA durchgeführt worden. Bei Patienten mit SpA gibt es keine Studien, die den diagnostischen Nutzen der Sonographie bei peripherer Arthritis untersuchen. Klinisch ist bei V.a. eine periphere Arthritis die Durchführung einer Arthrosonographie hilfreich zur Abklärung, ob ein Erguss oder eine Synovialitis detektiert werden kann. Auch bei unklaren Gelenkbeschwerden, insbesondere bei Patienten mit Hüftschmerzen, kann die Durchführung einer Arthrosonographie wertvolle Hinweise für die Genese der Beschwerden erbringen (z. B. Nachweis einer Arthritis oder Bursitis).

Für die Wirbelsäule können mittels Sonographie kaum Aussagen getroffen werden. In kleinen Studien wurden kürzlich SI-Gelenke bewertet [281, 282]. Da bislang nur wenige Patienten unter kontrollierten Bedingungen untersucht worden sind, erfolgt hier noch keine Bewertung dieser neuen Methode.

Die Sonographie ist hilfreich im Aufdecken einer enthesialen Beteiligung bei SpA-Patienten und sensitiver als die klinische Untersuchung [35, 283]. Die Sensitivität der Sonographie wird bei Patienten mit SpA zwischen 55,7 und 76,5\% angegeben und die Spezifität zwischen 81,3 und 89,5\% [284, 285]. Die Hypervaskularisation im Bereich von Sehnenansätzen mittels Power-Doppler-Signal von mindestens einem Sehnenansatz hat die höchste diagnostische Aussagekraft (positive LR 4,1 [OR 14,1; $p<0,0001$ ]), negative LR 0,2 [284, 286]. Allerdings zeigt sich keine Korrelation zwischen im US dokumentierter Enthesitis und klinischer Krankheitsaktivität sowie bildmorphologisch nachgewiesener Sakroiliitis, wie in der Frühkohorte DESIR gezeigt werden konnte [287].

\subsubsection{Szintigraphie}

Der diagnostische Nutzen einer Szintigraphie ist bei Patienten mit axialer SpA gering [288, 289]. Die Sensitivität wurde für Patienten mit AS mit 51,8\% und für Patienten mit möglicher Sakroiliitis mit $49,4 \%$ angegeben. Die Spezifität lag für eine beidseitige Sakroiliitis bei 57,7\%, für einseitige Sakroiliitis bei 92,8\% und insgesamt für Sakroiliitis (ein- oder beidseitig) bei 50,5\%. Somit war die positive LR der Szintigraphie für Sakroiliitis nur 1,3.

\subsubsection{Computertomographie}

Die Computertomographie (CT) kann zur Beurteilung von strukturellen Veränderungen in den SI-Gelenken eingesetzt werden. Eine KM-Applikation ist in der Regel nicht erforderlich. In Kohorten mit definitiver AS wird die Sensitivität der CT zur Darstellung von postentzündlichen Strukturveränderungen bei Sakroiliitis mit $90 \%$ angegeben [42]. Die CT ist besonders sensitiv für die Detektion von Erosionen, Ankylose und Fusionen. In Kohorten mit V.a. eine axiale SpA wird die Sensitivität allerdings nur mit $49 \%$ angegeben bei allerdings hoher Spezifität zwischen 73 und $96 \%$ (je nach untersuchter Vergleichsgruppe [Kontrollen oder mechanischer Rückenschmerz]) $[42,290]$. Die gegenüber konventionellen Röntgenuntersuchungen höhere Strahlenexposition sollte bei der Indikationsstellung berücksichtigt werden. Eine kleine kontrollierte Studie zur PET/CT lässt keine Aussagen hinsichtlich der diagnostischen Aussagekraft zu [291].

\section{Empfehlung/Statement}

6-1

Bei Patienten mit Verdacht auf axiale Spondyloarthritis sollte eine Bildgebung der Sakroiliakalgelenke erfolgen. Abhängig von der Symptomdauer und unter Berücksichtigung von Alter und Geschlecht kann eine konventionelle Röntgenuntersuchung (Beckenübersicht) oder eine MRT-Untersuchung der Sakroiliakalgelenke mit Entzündungssequenz (STIR und/oder T1 nach Kontrastmittelgabe) erfolgen. Insbesondere bei jüngeren Erwachsenen mit kurzer Symptomdauer sollte die MRT bevorzugt werden. Empfehlungsgrad: Evidenz: Expertenkonsens 


\section{6-2}

Wenn strukturelle knöcherne Läsionen (Syndesmophyten) an der Wirbelsäule z. B. zur Beurteilung der Prognose erfasst werden sollen, sollte eine Röntgenuntersuchung des betroffenen WSAbschnitts erfolgen.

Empfehlungsgrad: -

Evidenz: Expertenkonsens

\section{6-3}

Röntgenuntersuchungen der Wirbelsäule im Krankheitsverlauf sollten nicht routinemäßig, sondern bedarfsorientiert erfolgen.

Empfehlungsgrad: $B$

Evidenz: 1+

\section{6-4}

Bei Patienten mit unauffälligem Röntgenbild der SI-Gelenke und mit starkem Verdacht auf eine axiale Spondyloarthritis soll eine MRT-Untersuchung der SI-Gelenke mit Entzündungssequenz (STIR und/oder T1 nach Kontrastmittelgabe) durchgeführt werden.

Empfehlungsgrad: A

Evidenz: 2b/3b

\section{Kommentar:}

Die Empfehlung wurde aufgrund der konsistenten Studienlage von einer "B"auf eine „ $A$ "-Empfehlung hochgestuft. Da ein unabhängiger Referenzstandard für eine Diagnosestudie Level 1 fehlt, wird eine andere Studienqualität nicht möglich sein.

\section{6-5}

Bei Patienten mit gesicherter axialer SpA und Rückenschmerzen soll beschwerdeorientiert eine MRT-Untersuchung des betroffenen Abschnitts des Achsenskeletts mit Entzündungssequenz (STIRE, T1, KM) zum Nachweis entzündlicher Veränderungen in der Wirbelsäule durchgeführt werden. Empfehlungsgrad: A

Evidenz: 2b/3b

\section{Kommentar:}

Die Empfehlung wurde aufgrund der konsistenten Studienlage von einer "B" auf eine " $A$ "-Empfehlung hochgestuft.

Da ein unabhängiger Referenzstandard für eine Diagnosestudie Level 1 fehlt, wird eine andere Studienqualität nicht möglich sein.

\section{6-6}

Ist bei Verdacht auf eine periphere Enthesitis eine Bildgebung erforderlich, sollten eine Sonographie oder eine MRT der betroffenen Region durchgeführt werden.

Empfehlungsgrad: $B$

Evidenz: 3b

\section{6-7}

Zur Diagnosestellung einer axialen SpA sollte die Szintigraphie nicht eingesetzt werden.

Empfehlungsgrad: $B$

Evidenz: 2a

\section{6-8}

Unter strenger Indikation kann zum Nachweis von Fusionen und Erosionen oder zur Differenzialdiagnose eine CTUntersuchung der SI-Gelenke indiziert sein.

Empfehlungsgrad: -

Evidenz: Expertenkonsens

\subsection{Laborparameter}

Der Stellenwert von HLA-B27 wird ausführlich im Abschn. 5.2.2.1 behandelt, da der Parameter ausschließlich bei der Diagnosestellung eine Rolle spielt und nicht zur Verlaufsuntersuchung herangezogen wird.

\subsubsection{Entzündungsparameter}

Nur ca. 40-60\% der Patienten mit axialer SpA weisen im Verlauf ihrer Erkrankung erhöhte CRP-Werte auf [74, 200, 292, 293]. Patienten mit einer AS haben tendenziell höhere CRP- und BSGWerte als Patienten mit nr-axSpA [74]. Der Nachweis von strukturellen Veränderungen in den SI-Gelenken im konventionellen Röntgenbild ist bei Patienten mit erhöhten CRP-Werten häufiger (adjustierte OR 1,85; $95 \%$-CI 0,96-3,56 [ $p=0,066])$ [74]. In der deutschen Inzeptionskohorte konnte gezeigt werden, dass initial erhöhte CRP-Werte bei Patienten mit axSpA das Risiko einer Röntgenprogression erhöhen [215, 294, 295].

In einer Metaanalyse wurde die klinische Relevanz von verschiedenen CRPWerten bei Patienten mit AS untersucht. Die Höhe des CRP korrelierte schwach positiv mit männlichem Geschlecht, Krankheitsaktivität (gemessen mit BASDAI) und Funktion (gemessen mit BASFI), alle $p<0,0001$ [293]. Mit einem hochsensitiven Standard (hs-CRP) gemessene CRP-Werte korrelieren allerdings besser mit klinischen Parametern für Krankheitsaktivität [296]. Bei ASPatienten wurde in dieser Studie eine signifikante Korrelation zwischen der Höhe des CRP und nächtlichen Rückenschmerzen $(\rho=0,3 ; p=0,012)$ und bei nr-axSpA-Patienten eine signifikante Korrelation zwischen der Höhe des CRP und Druckschmerz bei Patienten mit Enthesitis gefunden $(\rho=0,2 ; p=0,031)$. Die Relevanz des hochsensitiven CRP für die tägliche Praxis ist gegenwärtig noch unklar. Bei Patienten mit axSpA kann trotz normaler CRP-Werte gleichwohl eine aktive Erkrankung vorliegen - in diesen Fällen ist dann eine Bildgebung zielführender. In der oben zitierten Studie lag ein Trend für stärkere Schmerzen, mehr Steifigkeit und funktionelle Einbußen vor, wenn bei negativem CRP hohe hs-CRP-Konzentrationen vorlagen.

$\mathrm{Zu}$ beachten ist, dass die Höhe der CRP-Werte durch medikamentöse Therapie beeinflussbar ist. Sowohl NSAR als auch, deutlich stärker, TNFi führen zu einer Senkung des CRP. Der Effekt der NSAR auf die Höhe des CRP ist bei Patienten mit erhöhtem CRP stärker ausgeprägt. Das Ansprechen auf eine Therapie 
mit TNFi ist bei Patienten mit erhöhten CRP-Werten höher als bei Patienten mit normalen Werten (OR 2,8; $95 \%$ CI 1,3-5,7; adjustiert auf Alter und Geschlecht) [297].

Die Wertigkeit der BSG wurde bei Patienten mit SpA bisher nur in wenigen Studien untersucht. Die Studien zur Wertigkeit von BSG und CRP für die Patientenselektion und zur Überwachung von Patienten unter Therapie mit TNFi konnten keinen Vorteil für die Bestimmung der BSG gegenüber dem CRP nachweisen [297].

Es fehlen Studien, die untersuchen, wie häufig Entzündungsparameter bei Patienten mit axialer SpA bestimmt werden sollen. Die Häufigkeit der Untersuchung wird daher auf individueller Basis gewählt in Abhängigkeit von Symptomen, allgemeinem Gesundheitszustand des Patienten und von der Medikation.

In einzelnen Studien wird die diagnostische Wertigkeit von Calprotectin im Serum bei Patienten mit SpA untersucht [298]. Kleinere Kohortenstudien zeigen eine positive Korrelation zwischen erhöhten Calprotectin-Spiegeln und einer intestinalen Entzündung, wie dies bei $\mathrm{Pa}$ tienten mit gesicherter CED ja ebenfalls beschrieben ist [299]. Die Bestimmung des Calprotectins ist bei Patienten mit axSpA in der klinischen Routine nicht gebräuchlich. In die Bewertung des Calprotectin-Spiegels muss die Beeinflussung durch externe Faktoren (z. B. NSAR-Therapie) mit einfließen.

\section{Empfehlung/Statement}

\section{6-9}

Zur Erfassung und Überprüfung der Krankheitsaktivität bei Patienten mit axialer SpA sollten CRP und/oder BSG bestimmt werden.

Empfehlungsgrad: $B$

Evidenz: 2b

\section{Kommentar:}

Es gibt keine Studien, die überprüfen, mit welcher Methode (Labor/MRT) die Krankheitsaktivität erfasst werden sollte.

\section{Krankheitsaktivität und Prognose der SpA}

\section{Schlüsselfrage 5}

- Welche Fragen oder Maßnahmen eignen sich am besten zur Selektion/zur intensiveren Diagnostik/möglichen Überweisung von Patienten mit chronischen Rückenschmerzen?

Da sich die Leitlinie sowohl an Ärzte der hausärztlichen Versorgung als auch an Rheumatologen richtet, stehen zur Beantwortung dieser Schlüsselfrage für den einzelnen Arzt jeweils andere Aspekte im Fokus. So sind z.B. Patienten mit hoher Krankheitsaktivität Kandidaten für eine intensivere Diagnostik und Überwachung - daher wird die Erfassung und Messbarkeit der Krankheitsaktivität in diesem Kapitel näher erläutert. Die Erläuterung der diagnostischen Maßnahmen findet sich im Abschn. 6.1 und 6.2. Zur Abschätzung des Risikos, einen schweren Krankheitsverlauf zu erleiden, eignen sich Prognosefaktoren, die (und deren Wertigkeit) im zweiten Abschnitt dieses Kapitels unter besonderer Berücksichtigung der röntgenologischen Progression vorgestellt werden.

Die Schlüsselfrage impliziert, dass auf das Stadium bzw. auf die Schwere der Erkrankung eingegangen werden soll. Allerdings ist hierbei grundsätzlich zu beachten, dass es keine internationale Übereinstimmung hinsichtlich einer Definition für die Schwere der Erkrankung („severity“) bei Patienten mit axialer SpA gibt.

\subsection{Krankheitsaktivität}

Mit dem Begriff Krankheitsaktivität wird das Ausmaß der entzündlichen Aktivität beschrieben. Das Ausmaß der entzündlichen Aktivität kann grundsätzlich sowohl durch die klinische Untersuchung als auch durch Laborparameter oder durch Bildgebung evaluiert werden. Zur Erfassung der Krankheitsaktivität stehen neben Ergebnissen der klinischen Untersuchung, der Bildgebung und der Labordiagnostik 2 validierte Messinstrumente zur Verfügung: zum einen der vom Patienten selbst auszufüllende BASDAI (Bath Ankylosing Spondylitis
Disease Activity Index) und zum anderen der neu entwickelte ASDAS (Ankylosing Spondylitis Disease Activity Score), der auf einigen BASDAI-Fragen beruht und das CRP einschließt [67, 300].

\subsubsection{Bath Ankylosing Spondylitis Disease Activity Index (BASDAI)}

Der BASDAI gehört zu den Bath-Indizes (s. Abschn. 3.1.2.2.1). Der BASDAI erfasst die Krankheitsaktivität der $\mathrm{Pa}$ tienten, indem diese 6 Fragen nach Müdigkeit, Rückenschmerzen, Schmerzen in peripheren Gelenken und nach Morgensteifigkeit auf einer numerischen Ratingskala zwischen 0 und 10 beantworten [67, 301]. Der BASDAI hat einen Wert zwischen 0 (niedrige Krankheitsaktivität) und 10 (hohe Krankheitsaktivität). Die Übereinstimmung des Messinstrumentes zwischen verschiedenen Anwendern liegt zwischen 0,87 und 0,94 und ist damit ausreichend hoch. Eine klinisch wichtige Verbesserung $(\mathrm{MCID}=$, minimum clinically important difference“) ist mit 1,0 schmal [302]. Zur Abgrenzung hoher und niedriger Krankheitsaktivität wurde willkürlich vor Jahren ein BASDAI von 4 festgelegt [303]. In der klinischen Anwendung ist die hohe intraindividuelle Variabilität des BASDAI-Summenscores zu berücksichtigen [304]. Nur ca. 30\% der Patienten mit AS haben auch eine periphere Arthritis (BASDAI-Frage 3) bzw. Enthesitis (BASDAI-Frage 4). Die Evaluation einer Mini-BASDAI-Version, bei der die Fragen 3 und 4 den Patienten nicht gestellt wurden, bot jedoch keine Vorteile gegenüber der herkömmlichen Version [305]. Die BASDAI-Summenwerte korrelieren gut mit Schmerzen und Steifigkeit [210]. Allerdings grenzt der BASDAI-Schwellenwert von 4 nicht zuverlässig Patienten mit viel und wenig Entzündung in der Wirbelsäule ab [270].

\subsubsection{Ankylosing Spondylitis Disease Activity Score (ASDAS)}

Der ASDAS wurde in Analogie zum DAS-

28 der rheumatoiden Arthritis entwickelt - ein „zusammengesetztes Instrument, welches aus 3 von 6 BASDAI-Fragen (BASDAI 2 Rückenschmerz, BASDAI 3 Gelenkschwellung, BASDAI 6 Dauer Morgensteifigkeit) sowie dem CRP und 


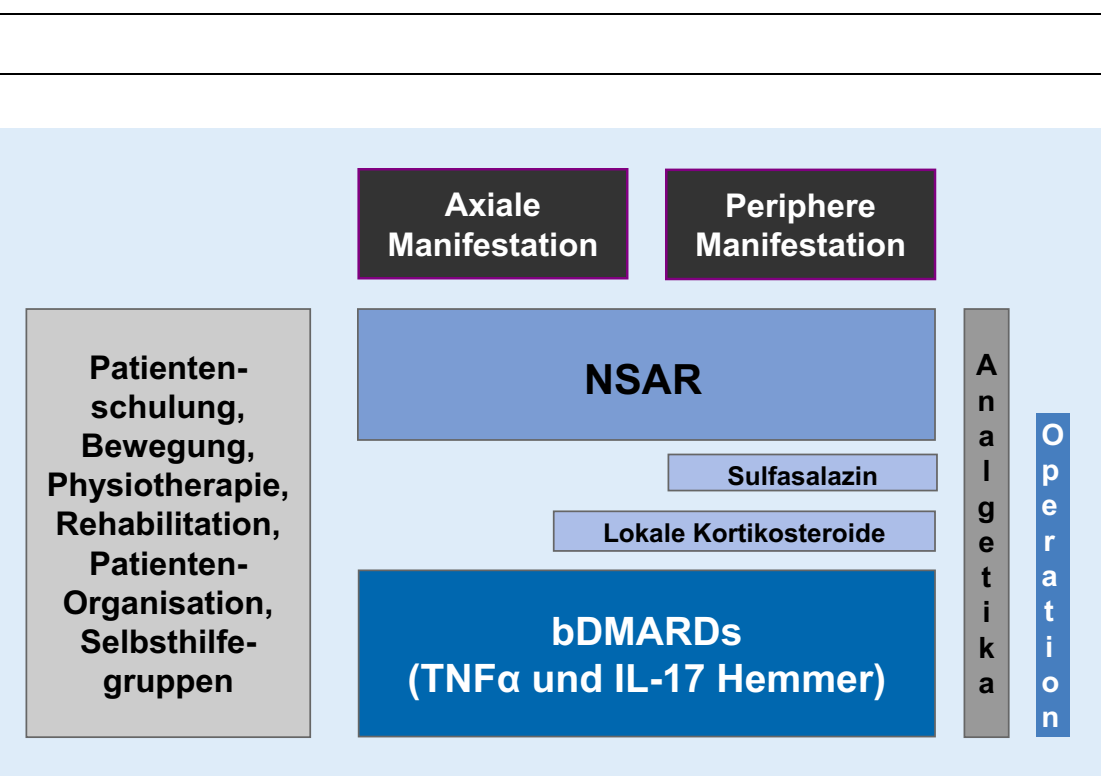

Abb. 3 ^ ASAS/EULAR-Empfehlung für die Behandlung der axialen Spondyloarthritis. (Publikation mit Genehmigung durch ASAS [www.asas-group.org])

dem Patientenglobalurteil besteht [300]. Bezüglich der CRP-Werte ist aufgrund einer Auswertung der DESIR Kohorte festgelegt worden, dass bei normwertigem CRP (bzw. hs-CRP $<2 \mathrm{mg} / \mathrm{l}$ ) mit einem Wert von $2,0 \mathrm{mg} / \mathrm{l}$ zu rechnen ist." [306]

Der ASDAS kann Werte zwischen 0 und 10 annehmen. Die Schwellenwerte wurden auf Datenbasis und per Expertenkonsensus definiert mit: $<1,3=$ inaktive Erkrankung bzw. Remission, $1,3-2,0=$ niedrige, $2,1-3,4=$ hohe und $\geq 3,5=$ sehr hohe Krankheitsaktivität [307]. Eine klinisch wichtige Verbesserung (MCID) wurde mit $\geq 1,1$ Punkten festgelegt, eine bedeutende Verbesserung („major improvement“) mit $\geq 2,0$ Punkten [307]. Mit Anwendung des ASDAS kann gut zwischen hoher und niedriger Krankheitsaktivität differenziert werden [308-310]. Es ist jedoch zu berücksichtigen, dass die Patientenpopulation, die mit Schwellenwert BASDAI $\geq 4$ oder ASDAS $\geq 2.1$ erfasst werden, nicht deckungsgleich sind. Bei 568 Patienten mit einem BASDAI $<4$ aus dem REGISPONDER-Register hatten 210 Patienten einen ASDAS $\geq 2,1$ und 16 Patienten einen ASDAS $\geq 3,5$ [311]. Umgekehrt gab es jedoch keine Patienten mit einem BASDAI $\geq 4$, die einen ASDAS $<1,3$ aufwiesen. Die diskriminatorische Kapazität des ASDAS unterscheidet sich nicht zwischen ASund nr-axSpA-Patienten [312]. Wie auch bei anderen PRO dokumentiert, liegen die ASDAS Scores für Frauen höher als die für Männer, welches jedoch aufgrund der unterschiedlichen Beantwortung der BASDAI Fragen zustande kommt [313].

Daten der deutschen Kerndokumentation zeigen, dass der Anteil von Patienten mit hoher Krankheitsaktivität (BASDAI $\geq 4,0$ ) von $37 \%$ im Jahr 2000 auf $19 \%$ im Jahr 2012 gesunken ist [314]. Parallel hierzu stieg der Anteil von Patienten mit guter Funktionsfähigkeit $(\mathrm{FFbH} \geq 75)$ von $36 \%$ im Jahr 2000 auf $49 \%$ im Jahr 2012.

\subsection{Prognosefaktoren}

Eine Vielzahl von Prognosefaktoren, die mit einem ungünstigen Verlauf assoziiert sind, ist für Patienten mit AS identifiziert worden. Allerdings wurden diese Faktoren überwiegend in retrospektiven Studien bzw. in Kohortenanalysen gefunden. Von den verschiedenen Faktoren ist die röntgenologische Progression am intensivsten untersucht worden. Des Weiteren wird im Abschn. 10 die Bedeutung des Rauchens als Prognosefaktor im Detail erläutert.

Prognostisch ungünstige Faktoren bei Patienten mit AS sind [70, 215, 224, 315-320]:

1. männliches Geschlecht,

2. Syndesmophyten bei der Erstvorstellung,

3. früher Beginn und lange Krankheitsdauer,
4. Hüftgelenkbeteiligung,

5. erhöhtes CRP,

6. röntgenologische SI-Gelenk-Veränderungen in den ersten 2 Jahren,

7. erhebliche Sakroiliitis in der MRT bei der Erstvorstellung.

Darüber hinaus sind Einschränkungen der körperlichen Funktionsfähigkeit durch körperlich belastende Arbeit, durch die Anzahl an Komorbiditäten und durch die Erkrankungsdauer möglich [70]. Die Durchführung regelmäBiger Bewegungsübungen sowie eine gute soziale Unterstützung führen offenbar zu einer besseren körperlichen Funktionsfähigkeit [319].

Patienten mit ungünstigen Prognosefaktoren sollten adäquat überwacht werden, um die Therapie dem Krankheitsverlauf anpassen zu können. Generelle Empfehlungen sind hier nicht möglich, da nicht nachgewiesen worden ist, dass eine engmaschige Überwachung in Kenntnis der schlechten Prognosefaktoren das Outcome verbessert. Die Überwachung der Erkrankung sollte deshalb in Abhängigkeit vom aktuellen klinischen Zustand des Patienten erfolgen, und die Häufigkeit des Monitorings sollte individuell unter Berücksichtigung der klinischen Symptomatik, des Schweregrads der Erkrankung und der durchgeführten Behandlung erfolgen (s. Abschn. 8.1).

\section{Therapien}

Die Behandlung von Patienten mit axSpA zeichnet sich durch ein multimodales Behandlungskonzept aus (• Abb. 3). Dies umfasst neben Schulungsmaßnahmen (Abschn. 10.1) insbesondere die Kombination von nichtpharmakologischen und pharmakologischen Maßnahmen (Abschn. 8.3 und 8.4) [21]. Im Laufe der Erkrankung können rehabilitative und invasive Maßnahmen wie Injektionen oder Operationen notwendig werden (Abschn. 8.7).

\section{Empfehlung/Statement}

Die meisten Therapiestudien sind bei $\mathrm{Pa}$ tienten mit AS durchgeführt worden. Bei der Übertragbarkeit der Ergebnisse auf die Gesamtgruppe der axialen SpA muss 
Tab. 9 Möglichkeiten der systematischen Erfassung von Therapiezielen

\begin{tabular}{|c|c|}
\hline Domäne & Instrument \\
\hline Allgemeines Patientenurteil & $\begin{array}{l}\text { Skala aür allgemeine Krankheitsaktivität in der vorhergehen- } \\
\text { den Woche }\end{array}$ \\
\hline Entzündungsparameter & CRP, BSG \\
\hline Ermüdbarkeit & BASDAI-Frage 1 (Müdigkeit und Erschöpfung) \\
\hline Körperliche Funktionsfähigkeit & BASFI \\
\hline Periphere Gelenke und Sehnen & $\begin{array}{l}\text { Anzahl geschwollener Gelenke ( } 44 \text { Gelenke) } \\
\text { Validierter Enthesitis-Score (z. B. MASES, San Francisco und } \\
\text { Berlin) }\end{array}$ \\
\hline Schmerz & $\begin{array}{l}\text { Skala für: } \\
\text { Nächtliche Schmerzen der Wirbelsäule in der vorhergehen- } \\
\text { den Woche wegen AS und } \\
\text { Schmerzen der Wirbelsäule in der vorhergehenden Woche } \\
\text { (s. oben) wegen AS }\end{array}$ \\
\hline Steifigkeit & $\begin{array}{l}\text { Skala }{ }^{a} \text { für Dauer Morgensteifigkeit der Wirbelsäule in der } \\
\text { vorhergehenden Woche }\end{array}$ \\
\hline Wirbelsäulenbeweglichkeit & $\begin{array}{l}\text { Thoraxexkursion: } \\
\text { Und modifizierter Schober } \\
\text { Und Okziput-Wand-Abstand } \\
\text { Und zervikale Rotation } \\
\text { Und laterale lumbale Flexion oder BASMI }\end{array}$ \\
\hline Arbeits- und Erwerbsfähigkeit & $\begin{array}{l}\text { Arbeitsunfähigkeitsdauer } \\
\text { Subjektive Arbeitsfähigkeit (Work-Ability-Index) }\end{array}$ \\
\hline
\end{tabular}

daher diskutiert werden, ob dadurch der Empfehlungsgrad automatisch reduziert werden sollte. Dies wurde von der Leitliniengruppe verneint, da es keine überzeugenden Hinweise darauf gibt, dass die therapeutischen Optionen bei Patienten mit axSpA im Gegensatz zu AS-Patienten schlechter wirken.

\section{8-1}

Das optimale Management für Patienten mit axialer SpA sollte eine Kombination aus nichtpharmakologischen und pharmakologischen Maßnahmen beinhalten.

Empfehlungsgrad: B

Evidenz: 1b

\section{Kommentar zu 8-1:}

Herabstufung der Empfehlung von Empfehlungsgrad „A“ auf „B", da die Kombination der beiden Maßnahmen in den klinischen Studien nicht der primäre Endpunkt war.

\section{8-2}

Die Therapiemöglichkeiten von Patienten mit axialer SpA können auch operative Maßnahmen umfassen.

Empfehlungsgrad: Statement Evidenz: -

\subsection{Therapieziele}

\section{Schlüsselfrage 1}

- Welches sind die vorrangigen Therapieziele (z. B. Schmerzreduktion, Verhinderung der röntgenologischen Progression, Arbeitsfähigkeit, Funktionserhalt)?

Wichtige Ziele für Patienten mit SpA sind die Schmerzreduktion, der Erhalt der körperlichen Funktionsfähigkeit, Reduktion der Steifigkeit, die Verhinderung struktureller Läsionen und der Erhalt der Arbeits- und Erwerbsfähigkeit. Ein Teil dieser Ziele kann systematisch erfasst werden (• Tab. 9; [321, 322]).

An zentraler Stelle in der Behandlung von Patienten mit axialer SpA stehen die Reduktion der Krankheitsaktivität und das Erreichen einer klinischen Remission. Remission wird für die axiale $\mathrm{SpA}$ entweder durch eine Kombination klini- scher Parameter („ASAS partial remission“: 4 Domänen [Schmerz, Funktion, Entzündung, Patientenurteil], kein Wert höher als 2 Einheiten) oder durch das Erreichen eines ASDAS-Schwellenwertes von $<1,3$ angegeben (s. Abschn. 7.1.2) [307, 323]. Prädiktoren für das Erreichen einer niedrigen Krankheitsaktivität bzw. Remission sind kurze Erkrankungsdauer, erhöhte Entzündungsparameter und erhaltene Funktionsfähigkeit [15, 324-328].

Die Verhinderung der röntgenologischen Progression in Gelenken und Wirbelsäule ist ebenfalls ein wichtiges Ziel in der Rheumatologie. Die Hemmung der röntgenologischen Progression ist im Gegensatz zum Effekt von Therapien auf das Entstehen von Erosionen bei der RA bei Patienten mit axialer SpA bisher noch nicht ganz überzeugend gezeigt worden.

\section{Empfehlung/Statement}

8-3

Die Therapie eines Patienten mit axialer SpA sollte immer wieder an den aktuellen Gesundheitszustand, die Auswirkungen der Behandlung und die gemeinsam vorab definierten Ziele angepasst werden (s. Präambel 2.5). Empfehlungsgrad: Evidenz: Expertenkonsens

\section{8-4}

Die Behandlung sollte fortwährend an ein festgelegtes Therapieziel angepasst werden. Dieses wird zwischen Arzt und Patient festgelegt und kann im Krankheitsverlauf adaptiert werden. Dabei liegt für das Erreichen einer Remission/niedrigen Krankheitsaktivität die größte Evidenz vor.

Empfehlungsgrad: -

Evidenz: Expertenkonsens

\subsection{Therapiestrategie}

\section{Schlüsselfrage 23}

- Wie wirken sich die verschiedenen

Facetten der Erkrankung auf die

Therapiestrategie aus?

Es gibt keine Studien, die verschiedene Therapiestrategien miteinander vergleichen. 


\subsection{Nichtpharmakologische Therapiemaßnahmen}

\section{Schlüsselfrage 15}

- Welche Effekte haben physiotherapeutische Verfahren, und welche Behandlungsmethode erzielt die besten Ergebnisse und mit welcher Intensität?

\section{Schlüsselfrage 16}

- Welche Effekte haben Trainings- und Rehabilitationsprogramme?

Körperliche Aktivität stellt neben der gewöhnlich parallel laufenden medikamentösen Therapie eine wesentliche Säule im Behandlungskonzept der axialen SpA dar (-Abb. 3). Dabei bezieht sich der Begriff „körperliche Aktivität“ sowohl auf die Steigerung der Alltagsaktivität, die sportliche Betätigung oder Durchführung physiotherapeutischer Maßnahmen. Ziele der Bewegungstherapie sind nicht nur der Erhalt der körperlichen Beweglichkeit und die Verminderung der Steifheit, sondern auch die Schmerzreduktion, eine verbesserte Haltung, Koordination, Sturzprophylaxe und der Erhalt der funktionalen Gesundheit (s. Abschn. 9). Dabei ist ein erklärtes Ziel der Therapie, an die Ressourcenpotenziale der Patienten anzuknüpfen. Es ist wichtig, den Patienten zu vermitteln, dass die Bewegungsübungen regelmäßig durchgeführt werden sollen. Ein weiterer wichtiger Aspekt ist es, den Patienten darauf hinzuweisen, dass Bewegung im Alltag ein essenzieller Aspekt der Behandlung ist. Die EULAREmpfehlungen zur körperlichen Aktivität bei Patienten mit entzündlich rheumatischen Erkrankungen fassen die wesentlichen Empfehlungen zusammen [329]. Die körperliche Aktivität sollte sich auf die Bereiche kardiorespiratorisches Training, Widerstandsübungen, Dehnungen und Stabilisationsübungen erstrecken. Vor dem Hintergrund von Komorbiditäten bei Patienten mit axialer Spondyloarthritis sollte der Gesundheitszustand des Patienten vor Einleitung einer Physiotherapie bekannt sein und in die Überlegungen mit einfließen [330].

Da Studien nur bei Patienten mit AS durchgeführt worden sind, gibt es keine
Grundlage für Empfehlungen für Patienten mit nr-axSpA. Im Analogieschluss ist jedoch davon auszugehen, dass regelmäßige Bewegungsübungen auch bei $\mathrm{Pa}-$ tienten mit nr-axSpA sinnvoll sind. Zudem gibt es keine Untersuchungen zu der Frage, ob Patienten mit Steifigkeit wegen struktureller Veränderungen im Gegensatz zu Patienten mit vorwiegend entzündlichen Veränderungen von Maßnahmen der Physiotherapie profitieren.

Die Studienlage zu Bewegungstherapien ist auch bei AS, methodisch bedingt, eher spärlich. Bei vielen Studien fehlt eine Kontrollgruppe, oft ist die Studiendauer zu kurz und die Anzahl der Patienten zu gering, und nicht selten fehlt ein definierter Endpunkt. Die Studien zeigen, dass physiotherapeutische und rehabilitative Maßnahmen kurz- und mittelfristig wirksam sind [331, 332]. Es ist schwierig, die Ergebnisse der einzelnen Studien direkt zu vergleichen, zumal die Art und Weise der Interventionen und die verwendeten Outcome-Parameter meist unterschiedlich sind.

Insgesamt ist die Rate der physiotherapeutischen Verordnungen immer noch gering. In der französischen Frühkohorte lag sie bei $24 \%$ innerhalb der ersten 6 Monate nach Diagnosestellung [333]. Die Daten der deutschen Kerndokumentation zeigen, dass $47 \%$ der AS-Patienten Physiotherapie verordnet bekamen [334]. Die Rate an Verordnungen war bei Patienten mit Funktionseinbußen am größten.

\subsubsection{Bewegungstherapie}

8.3.1.1 Bewegungstherapie im Trockenen. Für Patienten mit AS sind folgende Bewegungstherapien im Trockenen beschrieben worden: angeleitete Einzelkrankengymnastik, Eigenübungsprogramm im Rahmen der häuslichen Bewegungstherapie, angeleitete Gruppentherapien und kombinierte Therapien. Eine Cochrane-Analyse zeigt, dass häusliche Bewegungstherapie oder angeleitete Übungen besser sind als keine Therapie, dass angeleitete Gruppentherapien besser sind als häusliche Übungen und dass kombinierte Therapien mit Übungen im Wasser und Übungen auf dem Trockenen gefolgt von Gruppentherapien besser sind als alleinige Gruppentherapie [331]. Eine Metaanalyse zeigt einen Einfluss angeleiteter Bewegungsübungen auf die Krankheitsaktivität („weighted mean deviation" $=-0,581 \quad[95 \%-\mathrm{CI}=-0,940$ bis -0,222]), auf die körperliche Funktionsfähigkeit („weighted mean deviation" $=-0,438 \quad[95 \%-\mathrm{CI}=-0,791$ bis $-0,085])$ und auf den Bewegungsumfang der Wirbelsäule („weighted mean deviation “ $=-0,513 \quad[95 \%-\mathrm{CI}=-0,948$ bis -0,078]) [335]. Dass Patienten mit AS von Übungsprogrammen profitieren, belegt ein systematisches Review, in der der Einfluss von Bewegungsübungen auf Schmerzen, Steifigkeit der Wirbelsäule, des Thorax und der Hüftgelenke, Krankheits- und Alltagsaktivität belegt wurde. Ebenso wurde der Patientenaufklärung sowie der aktiven Beteiligung und Motivation eine hohe Bedeutung zugemessen [336]. In einer kontrollierten Studie mit 70 Patienten konnte gezeigt werden, dass sowohl Übungen zur Steigerung der körperlichen Fitness als auch Dehnungsübungen die körperliche Funktionsfähigkeit bei Patienten mit AS positiv über 24 Wochen beeinflussen [337]. Die Durchführung eines häuslichen Übungsprogramms wirkt sich nicht nur auf die Krankheitsaktivität und die körperliche Funktionsfähigkeit aus, sondern auch auf depressive Symptome des Patienten [338]. Für ein kardiovaskuläres Fitnesstraining, das 3-mal pro Woche zusätzlich zu wöchentlichen Übungen zur Förderung der Beweglichkeit durchgeführt wurde, konnten im Vergleich $\mathrm{zu}$ alleinigen Bewegungsübungen eine höhere Fitness sowie eine Schmerzreduktion nachgewiesen werden [339]. In einer kleinen Studie mit 48 AS-Patienten pro Gruppe zeigte sich, dass ein multimodales Training mit Pilates, McKenzieund Heckscher-Übungen im Vergleich $\mathrm{zu}$ einem klassischen Bewegungsprogramm die körperliche Funktionsfähigkeit, die Krankheitsaktivität und die Lungenfunktion verbessert [340]. Die Effektivität von Pilates-Training in Bezug auf eine Besserung der körperlichen Funktionsfähigkeit zeigte sich auch in einer weiteren, kleineren Studie mit insgesamt 55 Patienten [341].

Jedoch zeigen Versorgungsstudien, dass regelmäßige Bewegungstherapie 
nur von ca. einem Drittel der Patienten auch wirklich konsequent durchgeführt wird - unabhängig von einer begleitenden Therapie mit TNFi [342]. In einer norwegischen Kohortenstudie wurden der Grad der körperlichen Aktivität sowie der Energieverbrauch in metabolischen Äquivalenten erhoben [343]. Hier zeigte sich, dass alle Patienten mit AS gegenüber gesunden Kontrollen einen geringeren Energieverbrauch angeben und dass Patienten mit einer hohen Krankheitsaktivität den niedrigsten Energieverbrauch angeben (Median MET/Woche 4,300 in der Kontrollgruppe und 3,073 bei hoher- bzw. 4,290 bei niedriger Krankheitsaktivität $[p=0,02])$. In einer weiteren RCT wurde die Wirksamkeit eines 3-wöchigen multidisziplinären Rehabilitationsprogramms gegenüber einer üblichen Therapie bei Patienten mit AS verglichen. Die Ergebnisse zeigten signifikante Verbesserung von Krankheitsaktivität, Schmerzsituation, Wohlbefinden und der körperlichen Funktionsfähigkeit [344]. Der nachhaltige Effekt von Rehabilitationstraining in Form von Kräftigung, Dehnungen, Herz-Kreislauf-Training, Wirbelsäulen-, Thorax- und Hüftgelenkmobilisationen wurde in einer kontrollierten, klinischen Untersuchung untersucht. Im Vergleich $\mathrm{zu}$ einer Kontrollgruppe mit einer Verhaltensschulung konnte nach 12 Monaten eine verbesserte Wirbelsäulenbeweglichkeit, Thoraxexpansion und Krankheitsaktivität nachgewiesen werden [345] (s. auch Abschn. 8.7).

8.3.1.1.1 Eigenübungsprogramm im Rahmen der häuslichen Bewegungstherapie verglichen mit keiner Thera-

pie. Die Mehrzahl der Studien zeigt eine Verbesserung der körperlichen Funktionsfähigkeit nach 2 bis 4 Monaten sowohl in der Behandlungs- als auch in der Kontrollgruppe [331, 346-350]. In einer Metaanalyse von 6 RCTs mit insgesamt 1098 Patienten wurden durch Bewegungsprogramme im häuslichen Bereich größere Effekte in Bezug auf Krankheitsaktivität, Funktionsfähigkeit und depressive Symptome nachgewiesen, die allerdings nur für die Funktionsfähigkeit von klinischer Relevanz waren [338]. Die regelmäßige Bewegungstherapie bestand aus angeleiteten Übungen durch Physiotherapeuten, die die Patienten in der Regel regelmäßig (Schwankung zwischen täglich bis 2-mal wöchentlich) selbstständig zu Hause durchführen sollten. Die Kontrollgruppe bestand aus Patienten, die eine Gruppentherapie oder keine bestimmte Bewegungstherapie durchgeführt haben. Eine Metaanalyse aus 3 kontrollierten Studien fand ebenfalls nach Bewegungsübungen bessere Funktionsfähigkeit, Krankheitsaktivität und Beweglichkeit gegenüber alltäglichen Bewegungsaktivitäten [335]. Im Widerspruch hierzu steht eine Studie, die keine Verbesserung der körperlichen Funktionsfähigkeit zeigte [351]. Neue Entwicklungen der Exergames (körperliche Übungsprogramme in Verbindung mit spielerischen Computeranimationen) haben verglichen mit Kontrollen ohne Exergames-Übungen höhere Effekte auf die körperliche Funktionsfähigkeit gezeigt [352]. Es überwiegen erste Studien, die eine Verbesserung der pulmonalen Funktion durch regelmäßige Bewegungstherapie zeigen.

In einer Studie wurde mittels einer experimentellen Methode (Global Postural Reeducation[GPR]-Methode) eine Verbesserung der pulmonalen Funktionsparameter erreicht, während in einer anderen Studie keine Verbesserung festgestellt werden konnte [347, 349]. Die Diskrepanz kann jedoch auch auf die relativ kurze Beobachtungszeit (12 bzw. 8 Wochen) zurückzuführen sein.

Das aktuelle Cochrane-Review zur Physiotherapie bei AS kommt hinsichtlich der Physiotherapie bei AS zu ähnlichen Schlussfolgerungen [331, 353, 354]. In 3 Studien wurden eindeutige Verbesserungen der körperlichen Funktionsfähigkeit festgestellt [353-355], aber nur in einer konnte gezeigt werden, dass auch die Schmerzen durch die Bewegungstherapie abnahmen [355]. In einer Studie wurde insbesondere eine Verbesserung der Thoraxbeweglichkeit und des Hinterkopf-Wand-Abstandes erzielt (gewichtete mittlere Differenz 1,46; 95\%-CI 0,29-2,63) [354]. In einer weiteren Arbeit konnte die erreichte Funktionsverbesserung durch eine dauerhafte gering intensive Behandlung, bei der im Mittel nur 1,5 Visiten durch den
Physiotherapeuten in 4 Monaten erforderlich waren, aufrechterhalten werden [356].

8.3.1.1.2 Einzeltherapie verglichen $\mathrm{mit}$ Gruppentherapie. Eine Studie, die Effekte einer Gruppentherapie mit denjenigen von häuslichen Übungen verglich, fand in keiner Gruppe eine Verbesserung von körperlicher Funktionsfähigkeit und Schmerzen nach einer Therapie von 6 Wochen [357]. Für das allgemeine Gesundheitsgefühl der Patienten waren Bewegungstherapie in der Gruppe mit Maßnahmen physikalischer Therapie besser als eine Individualtherapie [358]. Im Vergleich zu einem häuslich durchgeführten Eigenübungsprogramm zeigte sich in einer Studie nach einem 3-wöchigen Programm mit Bewegungstherapie in der Gruppe und Hydrotherapie eine kurzfristige Verbesserung der Schmerzen, dieser Effekt hielt aber nicht über 6 Monate hinaus an [359]. Eine andere randomisierte Studie zeigte keinen zusätzlichen Effekt einer solchen Intervention auf Schmerzen und Funktion [360]. Bewegungstherapie in der Gruppe wurde darüber hinaus als kosteneffektiv bewertet [361].

\subsubsection{Bewegungstherapie im Wasser} (Balneotherapie). Studien, die Ergebnisse von Maßnahmen im Wasser berichten, werden häufig in Thermalbädern durchgeführt. Innerhalb der Studien gibt es für die Patienten häufig kombinierte Anwendungen mit aktiven Übungen in warmem Wasser und passiven Anwendungen wie Fangopackungen. Teilweise werden die Ergebnisse während eines stationären Rehabilitationsprogrammes erhoben, was die Analyse der einzelnen Effekte erschwert [351, 362, 363]. In 2 der 3 Studien erhielten die Patienten zusätzlich eine Therapie mit TNFi [351, 362]. In einer Studie verbesserte sich nur die Therapiegruppe an den 3 Untersuchungszeitpunkten (T1 nach den Anwendungen, T2 nach 3 Monaten und T3 nach 6 Monaten) bezüglich der körperlichen Funktionsfähigkeit (gemessen mit BASFI). Die Krankheitsaktivität (gemessen mit BASDAI) war nach 6 Monaten aber in beiden Gruppen nicht signifikant unterschiedlich gegenüber Baseline. Ein Gruppen- 
vergleich wurde in dieser Studie nicht durchgeführt. Dagegen besserten sich in einer anderen Studie nach 6 Monaten nicht der BASFI, sondern nur die Parameter für Lebensqualität [351]. In einer weiteren Studie wurde eine Therapie mit Stangerbädern in Kombination mit Bewegungstherapie über eine Periode von 3 Wochen mit einer Gruppe verglichen, die nur Bewegungstherapie über eine Periode von 3 Wochen durchgeführt hatte [363]. Hierbei zeigte sich in beiden Gruppen eine signifikante Verbesserung in BASFI und BASDAI, jedoch nicht im BASMI. Die Prüfung des Gruppenunterschieds zeigte eine stärkere Verbesserung für die kombinierte Therapiegruppe. In einem Cochrane-Review zeigten die eingeschlossenen Studien keine Unterschiede zwischen der Balneotherapiegruppe und der Vergleichsgruppe, weder bei Beweglichkeit noch bei Schmerzen und Steifheit [331, 364, 365].

\subsubsection{Manuelle Therapie}

Manuelle Mobilisation kann zu einer verbesserten Körperhaltung und zu einer Zunahme der Wirbelsäulen- und Thoraxbeweglichkeit führen (Thoraxexpansion) [349]. In der manuellen Medizin kommen als Behandlungstechniken Weichteiltechniken, Mobilisation, Manipulation und neuromuskuläre Therapie zur Anwendung. Aufgrund des geringeren Kraftimpulses mit hoher Geschwindigkeit und kleiner Amplitude stellt die Manipulation eine Besonderheit dar und sollte an der Wirbelsäule bei Patienten mit axialer SpA nicht eingesetzt werden. Die manuelle Mobilisationsbehandlung löst sensomotorische (wie beispielweise veränderte Schweißsekretion, Durchblutung und Tonusregulation) und neurophysiologische (z. B. Schmerzverarbeitung) Effekte aus. Ziel dieser Methode ist die Normalisierung und Ökonomisierung von Körperfunktionen. Die oben zitierte, kleine Studie ( $n=34$ Patienten mit AS) von Widberg et al. enthält keine Angaben $\mathrm{zu}$ vorbestehenden funktionellen Einbußen, die als Einschlusskriterium gewählt wurden. Insbesondere gibt es keine Angaben zu bereits bestehenden Strukturveränderungen. Der BASFI in der Erstuntersuchung lag bei 3,2 $\pm 1,75$; ein Schmerzlevel wird nicht angegeben. Die Studie zeigt eine Verbesserung der Körperhaltung und eine Verbesserung im BASMI, die sich nach einer 8-wöchigen Therapie zeigte. Außerhalb dieser Studie und einer Studie von 2005 gibt es lediglich Fallberichte zum Themenkreis der manuellen Medizin [366]. Diese beschränken sich auf manuelle Therapien am Achsenskelett und umfassen weitgehend Mobilisationstechniken und zielen weniger auf Manipulationen ab. Manipulationen am SI-Gelenk spielen lediglich in der frühen Erkrankungsphase eine Rolle.

Generell sollte ein erfahrener Therapeut die genannten Techniken durchführen. Es können aufgrund der Datenlage keine Aussagen zur Selektion der Patienten, zur Häufigkeit der Verordnungen und zur Dauer der Maßnahme getroffen werden. Die zitierte Studie basiert auf einer Studiendauer von 8 Wochen.

\subsubsection{Hyperthermie/Kältetherapie}

Diese Verfahren sind in kleinen kontrollierten Studien untersucht worden. In einer niederländischen Studie mit 17 ASPatienten führte eine Therapie mit 2 Saunagängen $\left(55^{\circ} \mathrm{C}\right) 2$-mal wöchentlich über einen Zeitraum von 4 Wochen $\mathrm{zu}$ einer Reduktion der Steifheit und Schmerzen unmittelbar nach den Saunagängen [367]. Dieser Effekt bestand ebenfalls in der Kontrollgruppe mit RA-Patienten, in beiden Gruppen hielten die Effekte aber nicht dauerhaft an. Eine milde Hyperthermie im Vergleich zu einer gesunden Kontrollgruppe führt bei Patienten mit AS ohne Entzündungsschub zu signifikant niedrigeren Zytokinspiegeln 6 und $12 \mathrm{~h}$ nach einem Überwärmungsbad [368].

Die Ganzkörperkältetherapie wurde bezüglich der Schmerzreduktion von ASPatienten in einer kleinen kontrollierten Studie als nicht effektiv angesehen [369].

\subsubsection{Elektrotherapie, Magnetfeld- therapie und Ultraschall}

Eine sehr begrenzte Anzahl an Studien nimmt zu elektrotherapeutischen Verfahren Stellung. Stangerbäder in Kombination mit Bewegungstherapie über 3 Wochen führten gegenüber der Gruppe mit Übungen ohne Stangerbäder zu einer Verbesserung von BASMI, BASFI, BASDAI und ASQoL [363].

Die transkutane elektrische Nervenstimulation (TENS) im Vergleich zu einer simulierten TENS-Behandlung über 3 Wochen ergab im Rahmen einer randomisierten klinischen Studie keine signifikante Kurzwirksamkeit hinsichtlich Schmerzen in der Behandlungsgruppe [370].

Für eine 20-minütige Magnetfeldtherapie der Hüftregionen $(2 \mathrm{~Hz})$ zusätzlich zu Kurzwellentherapie und Bewegungsübungen an 15 Tagen wurde im 6-monatigen Verlauf bei 66 AS-Patienten kein Unterschied gegenüber Plazeboultraschall bei sonst gleicher Kombinationstherapie festgestellt [371].

In einer kleinen kontrollierten Studie mit 52 AS-Patienten wurde der additive Effekt von einer Ultraschaltherapie $(5 \mathrm{~Hz})$ der paravertebralen Muskulatur vor dem Hintergrund eines supervidierten Übungsprogramms untersucht [372]. Die Autoren kamen zu dem Schluss, dass die zusätzliche Ultraschalltherapie den Effekt der Übung bei Patienten mit AS erhöht.

\subsubsection{Ergotherapie}

Ergotherapie kann dazu beitragen, dass bei Patienten mit AS die Krankheitsaktivität abnimmt und die körperliche Funktionsfähigkeit zunimmt, wenn die Patienten eine Schulung zu gelenkschonendem Verhalten und Selbstmanagementmethoden anwenden [373]. 


\section{Empfehlung/Statement}

\section{8-5}

Patienten mit axialer SpA sollten zu Beginn und im Verlauf der Erkrankung auf die Wichtigkeit von Sport, Bewegung im Alltag und regelmäßige Bewegungstherapie hingewiesen und individuell beraten werden.

Empfehlungsgrad: $B$

Evidenz: 1/2+

\section{8-6}

Bewegungsübungen, die zu Hause durchgeführt werden, sind zwar effektiv, aber alleine nicht immer ausreichend. Angeleitete Bewegungstherapien (als Trocken- oder Wasserübungen), individuell oder als Gruppe, sollten zusätzlich zum häuslichen Bewegungsprogramm verordnet werden.

Empfehlungsgrad: B

Evidenz: 1/2+

\section{8-7}

Bewegungstherapien sollten zusätzlich zur medikamentösen Therapie (B) bzw. zu interventionellen Therapien (Expertenkonsens) erfolgen, da sie zu einer weiteren Verbesserung der Beweglichkeit und der Funktionsfähigkeit im Alltag führen.

Empfehlungsgrad: $B$

Evidenz: 1/2+

\section{Kommentar zu 8-5 bis 8-7:}

Die kontrollierten Studien ohne Randomisierung sind mehrheitlich über einen kurzen Zeitraum durchgeführt worden. Daher wurde der Empfehlungsgrad von "A" auf „B" herabgestuft. Die Aussagen beruhen auf Studien mit AS-Patienten, sodass sich der Empfehlungsgrad mindert, wenn er auf die Gesamtgruppe der axialen SpA übertragen wird.
8-8

Manuelle Therapie (Mobilisation) kann durchgeführt werden, um eine Verbesserung der Wirbelsäulenbeweglichkeit und eine verbesserte Körperhaltung zu erreichen.

Empfehlungsgrad: 0

Evidenz: 2

Kommentar zu 8-8:

Herabstufung des Empfehlungsgrades von "B" auf " 0 ", da nur eine schmale Datenlage vorliegt.

\section{8-9}

Manipulationen an der Wirbelsäule sollten nicht durchgeführt werden. Empfehlungsgrad: $B$

Evidenz: 2

\subsection{Medikamentöse Therapie}

\section{Schlüsselfrage 10}

- Welche medikamentöse Therapie sollte zu welchem Zeitpunkt bei Patienten mit axialer SpA eingesetzt werden und für wie lange?

\section{Schlüsselfrage 11}

- Zu welchem Zeitpunkt kann eine medikamentöse Therapie beendet werden?

\section{Schlüsselfrage 12}

- Nach welchem Zeitraum sollte bei den verschiedenen Medikamenten ein Therapieerfolg evaluiert werden?

\section{Schlüsselfrage 13}

- Welche unerwünschten Wirkungen von medikamentösen Therapien müssen im Langzeitverlauf beachtet und mit dem Patienten kommuniziert werden?

Das Ziel einer medikamentösen Therapie besteht in Schmerzreduktion, Verbesserung der Funktionsfähigkeit und der Steifheit sowie der Reduktion inflammatorischer Prozesse und in einer Hemmung der röntgenologischen Progression.

\subsubsection{Nicht-steroidale Antirheuma-} tika (NSAR)

8.4.1.1 Wirksamkeit. Nicht-steroidale Antirheumatika (NSAR) spielen in der Behandlung von Patienten mit axialer SpA eine zentrale Rolle. Für die AS liegt klare Evidenz vor, dass NSAR sowohl bei kurzfristiger als auch bei längerer Behandlungsdauer hinsichtlich der Linderung von Schmerzen und Steifigkeit an der Wirbelsäule und an peripheren $\mathrm{Ge}$ lenken wirksam sind [374-380]. In den Studien wird über einen mittleren Rückgang der Schmerzen um $30 \mathrm{~mm}$ auf einer visuellen Analogskala (VAS) $0-100 \mathrm{~mm}$ berichtet. Die Besserung setzt in der Regel in den ersten $48 \mathrm{~h}$ nach Einnahme der Medikation ein. Die Mehrzahl der Patienten (70-80\%) berichtet von einer guten bis sehr guten Besserung ihrer Symptome. In einer neueren kontrollierten Studie konnte gezeigt werden, dass unter einer NSAR-Monotherapie (in dieser Studie hatte die Vergleichsgruppe Infliximab erhalten) $35,3 \%$ der Patienten mit einer NSAR-Monotherapie den Endpunkt der partiellen Remission in Woche 28 erreichten (Infliximab-Gruppe allerdings 61,9\%) (s. Abschn. 8.4.2) [381]. Dieser Effekt ist unabhängig von der Gegenwart einer peripheren Arthritis. Patienten, die zusätzlich eine Synovitis hatten, zeigten aber weniger Besserung bei den Wirbelsäulenschmerzen [382]. Die Wirksamkeit der NSAR ist individuell unterschiedlich. In den Studien zeigte sich auf Gruppenebene eine höhere Wirksamkeit bei Gebrauch von höheren Dosen [378, 383]. Die Effektstärke für NSAR bezüglich "Schmerz" wurde in einer Metaanalyse mit -1,07 SMD (standardisierte mittlere Differenz) (95\%-CI -1,55 bis -0,58) angegeben [384]. In derselben Metaanalyse zeigte sich eine moderate Effektstärke bezüglich „körperlicher Funktionsfähigkeit" mit -0,54 SMD (95\%-CI -0,67 bis -0,42). Das Ausmaß der Effektstärke bei Patienten mit AS konnte in einem neueren Cochrane-Review, einer Metaanalyse und einer kontrollierten Studie bestätigt werden [379, 380, 385]. Daten zu Patienten mit nr-axSpA gibt es nicht, sodass medikamentöse Empfehlungen für diese Patientengruppe nur im Analogieschluss möglich sind. In ei- 
ner kontrollierten Studie mit Naproxen vs. Naproxen + Infliximab zeigte sich, dass $35,3 \%$ der Patienten im Naproxen-Monotherapiearm den Status einer klinischen Remission nach einer Therapiedauer von 24 Wochen erreichten, dabei fand sich kein Unterschied zwischen den Subgruppen AS und nr-axSpA [381].

In den meisten randomisierten Studien über NSAR in der Behandlung von ASPatienten werden verschiedene Substanzen miteinander verglichen. Bis jetzt gibt es hierbei allerdings keine klare Evidenz, dass ein NSAR besser ist als das andere. Bezüglich der Wirksamkeit der Medikation gibt es nach bisherigen Erkenntnissen keinen Unterschied zwischen traditionellen NSAR und Coxiben [386].

Eine Wirkung von NSAR auf in der MRT nachweisbare entzündliche Läsionen in der Wirbelsäule ist nicht nachgewiesen. In einer 6-wöchigen Studie mit Etoricoxib $90 \mathrm{mg} / \mathrm{Tag}$ bei Patienten mit aktiver AS traten nur geringfügige Änderungen der in der MRT sichtbaren entzündlichen Läsionen der Wirbelsäule auf - allerdings wurden hier auch nur relativ wenige Patienten eingeschlossen und vor allem solche, die klinisch auf NSAR nicht gut angesprochen hatten [279].

Ein geringer Einfluss einer NSARTherapie auf die Höhe von CRP und BSG ist nach Datenlage wahrscheinlich [293, 374, 383]. In einer gepoolten Analyse zeigte sich, dass die Änderung des CRP zwischen der NSAR- und der Plazebogruppe unterschiedlich war und dass der Therapieeffekt umso höher lag, je höher das CRP bei Einschluss in die Studie mit 851 AS-Patienten war [293]. In einer Metaanalyse zeigte sich allerdings kein sicherer Einfluss auf AkutePhase-Proteine [384].

Die Anzahl der zu behandelnden $\mathrm{Pa}$ tienten, um eine Verbesserung zu erzielen ( „number needed to treat" ${ }^{\text {" }}$ NNT), ist für eine Therapie mit Coxiben oder konventionellen NSAR klein und bewegt sich zwischen 2 und 3 [387]. In dieser Post-hoc-Analyse einer Studie mit 4 Armen (Etoricoxib $90 \mathrm{mg}$, Etoricoxib $120 \mathrm{mg}$, Naproxen $1000 \mathrm{mg}$, Plazebo) errechnete sich $\mathrm{zu}$ Woche 6 eine NNT für eine mindestens $30 \%$ ige Verbesserung des BASDAI von 2,0, 2,0 und 2,7 in den aktiven Gruppenarmen. Bei Patienten mit einer TNFi-Therapie konnte gezeigt werden, dass die Dosis der NSARMedikation durch die effektive Kontrolle der Krankheitsaktivität gesenkt werden kann (s. Abschn. 8.4) [388].

Der Therapieerfolg von einem einzelnen NSAR-Präparat kann in der Regel nach 1 bis 2 Wochen bei einer Dosierung in Maximaldosis beurteilt werden [378, 383]. In den ASAS-Empfehlungen zur TNFi-Therapie bei Patienten mit axialer SpA wird vor dem Beginn einer solchen Therapie eine Behandlung mit mindestens 2 NSAR über insgesamt 4 Wochen empfohlen, bevor eine nicht ausreichende Effektivität angenommen werden kann [389].

In der klinischen Praxis wird der behandelnde Arzt bei Unwirksamkeit eines Medikamentes häufig einen Präparatewechsel vornehmen. Studien über die Wirksamkeit eines Wechsels fehlen vollständig. In der oben zitierten DVMBBefragung gaben $20 \%$ der Patienten an, dass sie innerhalb des letzten Jahres vor Durchführung der Befragung mehr als ein NSAR-Präparat verwendet haben [390]. Bei Patienten mit noch nicht ganz beherrschten Schmerzen oder Schmerzen aus nichtentzündlichen Gründen können Schmerzmittel wie Paracetamol oder Opioide bzw. Opioid-ähnliche Medikamente berücksichtigt werden, wenn eine NSAR-Behandlung nicht angeschlagen hat, kontraindiziert war und/oder schlecht vertragen wurde [21]. Studien liegen hierzu allerdings nicht vor.

8.4.1.2 Therapiedauer. Die Datenlage bezüglich der optimalen Dauer der Medikation mit NSAR ist spärlich. Die Studien umfassen meist nur eine relativ kurze Studiendauer zwischen 6 und 12 Wochen, nur eine Studie ist in den letzten Jahren über einen Zeitraum von einem Jahr durchgeführt worden [378]. In einer offenen Befragung der Deutschen Vereinigung Morbus Bechterew (DVMB) gaben $71,4 \%$ der Patienten an, dass sie mehr als 4 Jahre mit NSAR behandelt wurden [390].

Es ist unklar, ob NSAR als Dauertherapie bei Patienten mit AS möglicherweise einen krankheitsmodifizierenden Effekt mit einer Reduktion der röntgenologi- schen Progression haben. In einer 2005 publizierten kontrollierten und randomisierten Studie wurde die Wirksamkeit von einer kontinuierlichen Therapie mit Celecoxib im Vergleich zu einer Bedarfstherapie untersucht. Hierbei ergaben sich deutliche Anhaltspunkte, dass die kontinuierliche Therapie einen günstigeren Einfluss auf die Röntgenprogression an der Wirbelsäule nach 2 Jahren hatte [391]. In einer Subgruppenanalyse zeigte sich nur bei Patienten mit erhöhtem CRP ein geringeres Fortschreiten der Röntgenprogression [294]. Diese Ergebnisse konnten in einer Analyse der deutschen Inzeptionskohorte bestätigt werden [295]. Allerdings konnte die Hemmung der radiologischen Progression unter einer kontinuierlichen NSARGabe in einer weiteren großen kontrollierten Studie nicht bestätigt werden [392]. In der Diclofenac-Gruppe mit kontinuierlicher Medikation war die mSASSS-Progression sogar numerisch höher $(1,28$ [95\%-CI 0,7-1,9]) als in der Diclofenac-bei-Bedarf-Gruppe mit 0,79 (95\%-CI 0,2-1,4).

8.4.1.3 Unerwünschte Wirkung. Die Sicherheit von NSAR ist besonders wichtig, wenn man in Betracht zieht, dass diese Behandlungsform bei einem nicht geringen Teil der Patienten über einen längeren Zeitraum durchgeführt werden muss. Die Studienlage hinsichtlich der Sicherheit von NSAR bei Patienten mit axSpA ist spärlich. In 2 Cochrane-Reviews zu NSAR bei Arthritiden bzw. bei axialer SpA wurden 17 bzw. 35 Studien eingeschlossen [379, 393]. Bei Patienten mit axSpA liegen kontrollierte Studien nur für Patienten mit AS vor. In dem aktuellen Cochrane-Review zur NSARTherapie bei axSpA wurde kein Unterschied zwischen traditionellen NSAR und COX-2-Hemmer hinsichtlich gastrointestinaler unerwünschter Ereignisse berichtet, insgesamt lagen in der Verumgruppe im Vergleich zu Plazebo mehr gastrointestinale unerwünschte Ereignisse (RR 1,92, $95 \%$ CI 1,41-2,61) [379]. In einer Studie wurde in den 3 Behandlungsgruppen (Diclofenac $75 \mathrm{mg} 2$-mal täglich, Celecoxib $200 \mathrm{mg}$ 1-mal täglich, Celecoxib $200 \mathrm{mg}$ 2-mal täglich) über eine Rate von unerwünschten Wirkun- 
gen von $54,8 \%$ berichtet [383]. Die Rate an schweren unerwünschten Ereignissen lag bei $20 \%$. Die Rate an gastrointestinalen unerwünschten Wirkungen lag in der Diclofenac-Gruppe $(28,4 \%)$ signifikant höher als in der Celecoxib-Gruppe (200 mg/Tag: $15 \%$, $400 \mathrm{mg} /$ Tag: 16,7\%) $(p=0,006)$. In einer kürzlich publizierten Kohortenstudie aus Schweden mit 21.872 Patienten mit SpA waren die Sicherheitsdaten vergleichbar mit denen publizierter Daten [394]. Das relative Risiko für arteriosklerotische Ereignisse unterschied sich in dieser Kohorte nicht zwischen COX-Hemmer und nichtselektiven NSAR: RR 1,0; $95 \%$-CI 0,7-1,5. Das relative Risiko für gastrointestinale Ereignisse war niedriger für Patienten, die keine NSAR erhalten haben: RR 0,5; $95 \%$-CI 0,4-0,7. Es liegen allerdings liegen umfangreiche Sicherheitsdaten zur NSAR-Langzeittherapie bei anderen Erkrankungen wie RA und Osteoarthrose vor [395, 396], diese Patientengruppen sind allerdings im Mittel durchweg deutlich älter als Patienten mit axialer SpA. Aufgrund der guten Effektivität der NSAR-Therapie bei axSpA wird das Nutzen-Risiko-Verhältnis der NSAR bei dieser Indikation für insgesamt günstig gehalten [397].

Neben Blutungsrisiken sind NSAR und Coxibe mit einem erhöhten kardiovaskulären Risiko assoziiert [398, 399]. Allerdings ist bei der Interpretation der kardiovaskulären Daten bei NSAR im Allgemeinen zu bedenken, dass bei Patienten mit AS eine erhöhte Mortalität für diejenigen Patienten gezeigt wurde, die wenig oder keine NSAR eingenommen hatten [128, 159]. Die norwegischen Forscher analysierten eine OR von 4,35 (95\%-CI 1,753-10,771) bei Patienten, die wenig oder keine NSAR eingenommen hatten, und die kanadischen Kollegen analysierten eine reduzierte HR von 0,1 (95\%-CI 0,01-0,61) für den Tod aufgrund eines vaskulären Ereignisses, wenn traditionelle NSAR eingenommen wurden.
Aufgrund des Sicherheitsprofils der NSAR-Präparate sollten die Dosierung und Dauer der Therapie kritisch fortwährend überprüft werden. Hierzu liegen DGRh-Empfehlungen zur Verordnung von NSAR vor [400]. Da es sich bei Patienten mit chronisch rheumatischen Erkrankungen um eine besondere $\mathrm{Pa}$ tientengruppe handelt, sind von einem multidisziplinären Expertengremium Empfehlungen zum Einsatz von NSAR und Coxiben veröffentlicht worden [401]. Bei Patienten über 60 Jahren und/oder weiteren gastrointestinalen Risiken sollte die Kombination mit einem Protonenpumpeninhibitor erwogen werden [402]. Zusätzlich wird bei Patienten mit Risikofaktoren wie gastrointestinale Blutung in der Anamnese, Komedikation mit Thrombozytenaggregationshemmer, oraler Antikoagulation, Bisphosphonaten, Colitis ulcerosa, Alkoholismus eine Kombination mit Protonenpumpenhemmern empfohlen [403].

Patienten, die eine Indikation zur Thrombozytenaggregationshemmung mit ASS haben, müssen diese Medikation ca. $1 \mathrm{~h}$ vor dem NSAR einnehmen, da ansonsten die Wirksamkeit der Thrombozytenaggregationshemmung reduziert sein kann [404, 405].

NSAR sind formal erst ab einer eGFR $<30 \mathrm{ml} / \mathrm{min}$ kontraindiziert. Da NSAR mit einem erhöhten Risiko für akutes Nierenversagen assoziiert sind und zu Progression der Niereninsuffizienz führen, sind eine individuelle Entscheidungsfindung und Monitoring notwendig [406, 407].

Ein Problem bei Patienten mit SpA kann selten darin bestehen, dass es durch den NSAR-Gebrauch zu einer Exazerbation einer Psoriasis vulgaris kommen kann [408]. Aufgrund dessen empfehlen die EULAR-Empfehlungen für die Behandlung der PsA NSAR als Erstlinientherapie [409].

\section{Empfehlung/Statement}

8-10

Nichtsteroidale Antirheumatika (NSAR) inklusive Coxibe sollen bei symptomatischen Patienten mit axialer SpA als Mittel der ersten Wahl eingesetzt werden.

Empfehlungsgrad: A

Evidenz: 1+

Kommentar zu 8-10:

Die Aussagen beruhen auf Studien mit AS-Patienten, sodass sich der Empfehlungsgrad mindert, wenn er auf die Gesamtgruppe der axialen SpA übertragen wird.

\section{8-11}

Die Dosierung und Therapiedauer der nichtsteroidalen Antirheumatika (NSAR) inklusive Coxibe richtet sich nach der Intensität der Beschwerden des Patienten. Empfehlungsgrad: Statement

Evidenz: -

\section{8-12}

Die Effektivität einer neu begonnenen Therapie mit nichtsteroidalen Antirheumatika (NSAR) sollte nach 2 bis 4 Wochen beurteilt werden. Weitere Kontrollen sollen individuell vereinbart werden.

Empfehlungsgrad: $\mathrm{B}$

Evidenz: 1+

\section{8-13}

Wenn ein NSAR nicht gewirkt hat, sollte ein zweites NSAR für weitere 2 bis 4 Wochen versucht werden Empfehlungsgrad: $B$

Evidenz: 1+

\section{Kommentar zu 8-13:}

Es gibt keine Strategiestudien, die den Ablauf verschiedener NSAR-Medikation miteinander vergleichen. Diese Schlussfolgerung beruht allein auf verschiedenen unabhängigen RCTs. Daher wurde die Empfehlung von Empfehlungsgrad ${ }_{\text {„A }} \mathrm{A}^{\prime \prime}$ auf „ $B$ " herabgestuft. 


\section{8-14}

Eine kontinuierliche Therapie mit nichtsteroidalen Antirheumatika (NSAR) ist indiziert, solange diese für eine gute Symptomkontrolle erforderlich sind. Empfehlungsgrad: Statement Evidenz: -

\subsubsection{Biologika („,biologic disease- modifying antirheumatic drugs" [bDMARDs])}

Patienten, die unter der Standardtherapie mit NSAR keine ausreichende Reduktion der entzündlichen Krankheitsaktivität erreichen, können Biologika verschrieben bekommen. In der französischen Frühkohorte haben innerhalb eines Jahres nach Einschluss in die Kohorte 23,4\% der Patienten Biologika verordnet bekommen [410]. Bei Patienten mit axSpA spielen die Zytokine Tumor-Nekrose-Faktor(TNF)- $\alpha$ und Interleukin-17 in der Pathogenese eine wichtige Rolle, die beide durch biotechnologisch hergestellte Substanzen geblockt werden können. TNF-Inhibitoren (TNFi) sind für die Gesamtgruppe der axSpA zugelassen, Interleukin-17-Inhibitoren im Moment nur für die AS. ${ }^{2}$

8.4.2.1 Tumor-Nekrose-Faktor-Inhibitoren (TNFi). TNFi sind bei Patienten mit axialer SpA klinisch effektiv und werden bei Patienten mit hoher Krankheitsaktivität eingesetzt [411-416]. Die pathophysiologische Rationale für den Einsatz von TNFi bei Patienten mit persistierend hoher Krankheitsaktivität beruht auf dem Nachweis von TNF$a$ in Biopsien aus entzündeten SI-Gelenken [417]. Die Wirkstärke („effect size“) der verschiedenen TNFi ist bei Patienten mit AS sehr hoch („effect size“ 0,89 und 1,3) [418, 419]. Die Anzahl der zu behandelnden AS-Patienten, um eine Verbesserung $\mathrm{zu}$ erzielen („number needed to treat ${ }^{\prime \prime}=\mathrm{NNT}$ ) ist klein und bewegt sich zwischen 2 und $3 \mathrm{zu}$ behandelnder Patienten [412, 418, 420]. Die NNT bei Patienten mit nr-axSpA unter einer Therapie mit TNFi liegt zwi-

\footnotetext{
${ }^{2}$ Die Zulassungsstudie zur nr-axSpA wird gerade durchgeführt.
}

schen 3,9 und 6,6 und somit etwas höher als bei AS-Patienten [421].

Die Wirksamkeit und Sicherheit der TNFi ist bei Patienten mit AS sehr gut belegt [250, 256-286, 422]. Patienten mit totaler Ankylose der Wirbelsäule profitieren ebenfalls von einer Therapie mit TNFi [423, 424]. Die Wirksamkeit und Sicherheit einer Gabe von TNFi ist bei Patienten mit nr-axSpA ebenfalls sehr gut belegt [425-428]. Die Indikation zur Einleitung einer Therapie mit TNFi umfasst jedoch zusätzlich $\mathrm{zu}$ einer klinisch definierten erhöhten Krankheitsaktivität noch den objektiven Entzündungsnachweis mit entweder erhöhtem CRP oder dem Nachweis einer kernspintomographisch darstellbaren aktiven Sakroiliitis (subchondrales Knochenmarködem) [21]. Die bei nraxSpA-Patienten bestehende geringere Effektstärke im Vergleich zur AS-Population wird durch verschiedene Autoren auf eine heterogenere Population der nr-axSpA-Patienten und auf geringere Krankheitsschwere in einigen der kontrollierten Studien zurückgeführt [413, 429]. In der Metaanalyse von Callhoff et al. zeigte sich nach Korrektur für das Publikationsjahr (als Proxy für die Krankheitsschwere) jedoch kein Unterschied zwischen der Effektstärke von TNFi bei AS und nr-axSpA [413]. Daten der Schweizer Biologikakohorte zeigen, dass die Wirksamkeit der TNFi-Therapie bei Patienten mit AS und nr-axSpA vergleichbar ist, wenn vergleichbare Ausgangsbedingungen (wie erhöhtes CRP) vorliegen [430]. Andere Kohorten konnten ebenfalls keinen Unterschied auf Gruppenniveau zwischen AS und nraxSpA in Bezug auf das Therapieansprechen von bDMARDs sehen [431]. Die Wirksamkeit einer TNFi-Therapie beginnt in der Regel früh, schon nach Tagen bis wenigen Wochen, bei den meisten Patienten ist dies spätestens in Woche 12 klar.

Prädiktoren für ein gutes Ansprechen auf eine TNFi-Therapie sind junges Alter bzw. kurze Krankheitsdauer, eine hohe Krankheitsaktivität (CRP, ASDAS), ein positiver HLA-B27-Befund, eine gute körperliche Funktionsfähigkeit (gemessen mit BASFI oder modifiziertem Schober), das Vorhandensein einer peripheren Arthritis und männliches Geschlecht [297, 326, 327, 432-435].

Die Indikation für eine Therapieeinleitung mit einem TNF-Inhibitor ist im Abschn. 8.4.2.4.1 näher erläutert.

Klinisches Bild: Die klinische Wirksamkeit von TNFi beginnt meist relativ schnell und hält bei einem größeren Teil der Patienten unter fortlaufender Therapie mehrere Jahre an $[152,253,381,388$, 424-426, 428, 436-460]. Fast alle kontrollierten Studien sind unter Einschluss von Patienten mit AS durchgeführt worden. Ausnahmen sind die Studie mit Certolizumab [460], die in der Gesamtgruppe axiale SpA durchgeführt wurde, und Adalimumab [428], die in der Indikation nraxSpA durchgeführt wurde. Biosimilardaten mit äquivalenten pharmakokinetischen Profilen liegen für Infliximab bzw. CT-P13 für die Indikation AS vor [461].

In den Zulassungsstudien der TNFi konnten eine Reduktion der Wirbelsäulenschmerzen und der Morgensteifigkeit sowie eine Verbesserung der Funktionsfähigkeit und eine Reduktion der Müdigkeit erzielt werden [153, 425, 427, 436, 438, 441, 448, 449, 454]. Studienergebnisse und die klinische Erfahrung zeigen, dass nicht alle Patienten mit axialer Symptomatik von einer Therapie mit den TNFi so ausreichend profitieren, dass die Schmerzmedikation vollständig abgesetzt werden kann. Im klinischen Alltag spielen bei der Bewertung eines nicht ausreichenden Ansprechens auf eine neu eingeleitete TNFi-Therapie Überlegungen $\mathrm{zu}$ Komorbiditäten wie vorbestehende degenerative Wirbelsäulenveränderungen bzw. eine generalisierte Schmerzsymptomatik eine Rolle. In einer Kohortenstudie konnte gezeigt werden, dass Patienten mit axSpA und FM eine niedrigere Ansprechrate auf eine TNFi-Therapie zeigen als Patienten ohne generalisiertes Schmerzsyndrom [125]. Auf die Dosisreduktion von NSAR unter einer suffizienten TNFiTherapie wurde bereits im Kapitel NSAR (s. Abschn. 8.4.1) eingegangen [388].

Neben axialen Symptomen klagen Patienten mit axialer SpA auch häufig über extraspinale Manifestationen, wie z.B. periphere Arthritis und/oder Enthesitis. Das Vorliegen einer peripheren Arthritis ist ein Prädiktor für die 
Fortführung einer TNFi-Therapie (HR 0,49 [95\%-CI 0,27-0,88]) [432, 433]. SpA-Patienten mit einer Enthesitis im Bereich der Fersenregion geben eine bessere globale Beurteilung nach einer 12-wöchigen Therapie mit Etanercept an als Patienten, die Plazebo erhalten haben [445]. Darüber hinaus bestehen bei einer peripheren Arthritis noch Therapieoptionen mit Sulfasalazin zur Beeinflussung einer peripheren Arthritis (s. Abschn. 8.4.3) und die Option einer Synovektomie, wobei hierzu allerdings keine Daten vorliegen.

Der Einfluss der TNFi auf pulmonale Funktionsparameter wurde in einer französischen Studie mit 82 AS-Patienten, die an einer fortgeschrittenen Ankylose der Wirbelsäule litten, geprüft. Es zeigte sich nach 3 Monaten ein positiver Trend für die mit Etanercept behandelte Gruppe, die Unterschiede waren jedoch nicht signifikant [424].

TNFi wirken sich günstig auf die Häufigkeit von Schüben einer AAU aus (s. Abschn. 8.4.2) [90-94]. Wie bei der Inzidenzrate der AAU unter verschiedenen TNFi gibt es Hinweise auf eine partiell unterschiedliche Wirksamkeit der TNFi hinsichtlich eines Krankheitsschubes im Rahmen einer CED [105]. In einer Metaanalyse von Studien mit AS-Patienten zeigte sich, dass es während einer Therapie mit Infliximab kaum zu Aktivität einer CED kam, während dies unter Etanercept häufiger beobachtet wurde [105]. Daher sollte bei begleitender CED eine Therapie mit monoklonalen Antikörpern einer Therapie mit dem Fusionsprotein Etanercept vorgezogen werden. Als weitere wichtige extraskeletale Manifestation bei Patienten mit axialer SpA gilt die Psoriasis vulgaris. Es gibt Berichte über paradoxe Reaktionen einer vorbekannten Psoriasis als auch Berichte über das Neuauftreten einer Psoriasis vulgaris [462]. Es fehlen Daten aus großen plazebokontrollierten Studien, sodass hier keine evidenzbasierte Stellungnahme möglich ist.

Patient-reported Outcome: Unter der Therapie mit TNFi steigt die Lebensqualität der Patienten stärker an als bei Patienten unter Plazebo [152, 463, 464]. Signifikante Veränderungen zeigen sich schon ab Woche 12 mit einer lang anhaltenden
Besserung im Langzeitverlauf [465]. Das Ausmaß der Veränderungen übersteigt die zu erwartende klinische Verbesserung mit einem MCID von 3,5 Punkten für den Gesundheitsstatus SF-36 [463]. Betrachtet man die Subskalen des SF36, verbessert sich die Summenskala für die körperliche Funktionsfähigkeit. Die Summenskala für die psychische Funktionsfähigkeit verändert sich nicht signifikant [463]. Trotz der Effektivität der Therapie mit Biologika bleiben die SF36-Werte auch unter Therapie unterhalb der der Vergleichspopulation [466].

Laborwerte: Die Wirksamkeit der TNFilässt sich auch in laborchemischen Verlaufsuntersuchungen nachweisen. CRP und BSG fallen unter einer Therapie mit einem TNFi parallel zum Rückgang der klinisch gemessenen Krankheitsaktivität [436, 438, 449]. Die Sensitivität und Spezifität des CRP für die Vorhersage eines ASAS-20-Ansprechens wurden in einer Kohorte mit 155 AS-Patienten mit $69 \%$ und $57 \%$ berechnet [297]. Patienten mit erhöhten CRP-Werten bei Beginn einer TNFi-Therapie sprechen generell besser auf eine Therapie mit TNFi an.

Die Verbesserung einer vorbestehenden Anämie durch eine Therapie mit TNFi konnte in einer plazebokontrollierten Studie mit Infliximab (ASSERT) gezeigt werden [467]. Eine Regressionsanalyse in dieser Studienpopulation zeigte, dass die Verbesserung des Hämoglobinwertes mit einer Verbesserung der körperlichen Funktionsfähigkeit (gemessen am BASFI) und der Müdigkeit einhergeht. Diese Daten sprechen dafür, dass die Hämoglobinwerte entzündungsbedingt niedrig waren.

Unter der Gabe von TNFi kann es zum Auftreten von antinukleären Antikörpern kommen. In einer französischen Kohorte mit 70 AS-Patienten ist das Auftreten von antinukleären Antikörpern beschrieben worden [468]. $\mathrm{Zu}$ Beginn der Therapie mit TNFi hatten $27 \%$ der AS-Patienten einen erhöhten ANA-Titer (Immunfluoreszenzmessung, Titer musste $>1: 250$ sein), im Laufe eines Jahres entwickelten dann $52 \%$ der Patienten einen erhöhten ANA-Titer. Allerdings zeigte kein SpA-Patient einen medikamentös induzierten Lupus.
Bildgebung: Die Wirksamkeit der TNFi lässt sich auch in MRT-Verlaufsuntersuchungen nachweisen. Bei Patienten mit axialer SpA zeigte sich nach 16 Wochen ein deutlicher Rückgang der Entzündung in den SI-Gelenken unter einer Therapie mit Infliximab [427]. Die Patienten mit einer höhergradigen Entzündung in den SI-Gelenken zeigten einen stärkeren Rückgang der Entzündung verglichen mit Patienten, die eine geringe Entzündung in den SI-Gelenken aufwiesen.

Unter Therapie mit TNFi kommt es zu einem mittels MRT nachweisbaren Rückgang der Entzündung auch in der Wirbelsäule, der nach 6 Monaten deutlicher ausgeprägt war als nach 6 Wochen $[275,277$, 469]. Jedoch können auch nach 2-jähriger Therapie mit einem TNFiimmer noch residuale entzündliche Läsionen in der Wirbelsäule nachgewiesen werden [275]. Die Bedeutung dieses Befundes für die Langzeitprognose ist unklar.

Im klinischen Alltag sind systematische Verlaufsuntersuchungen bei einzelnen Patienten nicht indiziert, diese sind für die weitere Erforschung jedoch wichtig und notwendig.

Es liegen derzeit keine kontrollierten Studien vor, die eine Hemmung der röntgenologischen Progression durch TNFi bei Patienten mit axSpA zeigen. Es existiert aber eine Reihe an Kohortenstudien, die eine Verlangsamung der Knochenneubildung nahelegen [216, 470-476]. Die Interpretation dieser Daten ist allerdings durch das Fehlen einer Kontrollgruppe erschwert.

Unter einer Therapie mit TNFi steigt die Knochendichte signifikant deutlicher an als bei Patienten, die Plazebo erhalten haben $[477,478]$. Dies hat für die Patienten eine große Bedeutung, da bekannt ist, dass die Patienten durch die Achsenskelettentzündung häufig eine Osteopenie aufweisen (s. Abschn. 3.1.1.4).

Erwerbstätigkeit: Die Effekte einer Therapie mit TNFi haben auch Einfluss auf die Erwerbstätigkeit der Patienten [479-483]. Schwedische Registerdaten zeigen, dass das Risiko, arbeitsunfähig zu sein, mit dem Beginn der Therapie mit TNFi abnimmt: Drei Monate vor Beginn der Therapie betrug die OR 8,0 (95\%CI 4,6-13,9) und 12 Monate nach Be- 


\section{Diagnose der axSpA durch den Rheumatologen}

und

Erhöhung von CRP und/ oder positives MRT und/ oder röntgenologische Sakroilititis*

und

Therapieversagen auf Standardtherapie:

Alle Patienten

- mindestens 2 NSAR über insgesamt 4 Wochen

Patienten mit predominant peripheren Manifestationen

- Eine lokale Steroidinjektion wenn möglich

- Normalerweise ein Therapieversuch mit Sulfasalazin

und

Hohe Krankheitsaktivität: ASDAS $\geq 2.1$ oder BASDAI $\geq 4$

und

Positive Meinung des Rheumatologen

Abb. $4 \Delta$ ASAS-Empfehlungen zur Anwendung von bDMARDs bei Patienten mit axialer Spondyloarthritis. (*Röntgenologische Sakroiliitis ist notwendig für Infliximab und IL17i) (Publikation mit Genehmigung durch ASAS, www.asas-group.org)

Erwäge die Fortführung von

bDMARDs nach mindestens 12

Wochen Behandlung, wenn
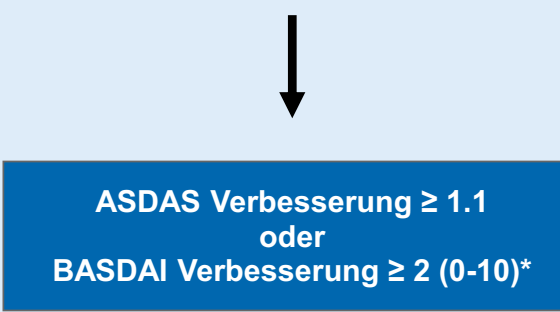

Positive

Expertenmeinung

des

Rheumatologen,

fortzuführen

Abb. $5 \Delta$ ASAS-Empfehlungen zur Überprüfung derbDMARD-Therapie. $\left({ }^{*}\right.$ Entweder ASDAS oder BASDAl können verwendet werden, jedoch dasselbe Messinstrument pro Patient) (Publikation mit Genehmigung durch ASAS, www.asas-group.org)

ginn der Therapie nur noch 4,0 (95\%-CI 2,1-6,3) [482]. Auch eine neuere Studie aus Schweden bestätigte den Rückgang der Arbeitsunfähigkeit innerhalb der ersten 2 Jahre nach Einsatz der TNFiTherapie [484]. Eine Studie aus England zeigte, dass arbeitslose Patienten unter einer TNFi-Therapie erneut ein Arbeitsverhältnis wieder aufnehmen können - allerdings umfasste die Kohorte nur 65 AS-Patienten [481]. Bei Patienten mit unsicherem Arbeitsverhältnis aufgrund der aktiven AS-Erkrankung reduzierte sich das Risiko, den Job zu verlieren, um $55 \%$ nach einer 12-wöchigen Therapie mit Etanercept [480].

8.4.2.2 Interleukin-17-Blocker. Secukinumab als Interleukin-17A-Hemmer zeigte in den Zulassungsstudien als auch in der Proof-of-concept-Studie eine gute Wirksamkeit und eine Sicherheitslage, wie sie von TNFi bekannt ist [485-488]. In der MEASURE 1 (i.v.-
Aufdosierung)-Studie lag die ASAS20 -Antwort in Woche 16 bei $61 \%$ bei einer Plazeboresponse-Rate von $29 \%$ [486]. In der MEASURE 2-Studie (75 und $150 \mathrm{mg}$ s.c. mit Aufdosierung über die ersten 4 Wochen) lag für die 150mg-Dosierung die ASAS-20-Antwort in Woche 16 bei TNFi-naiven Patienten bei $68,2 \%$ bei einer Plazeboresponse-Rate von $31,1 \%$ und bei TNFi-erfahrenen Patienten bei $50,0 \%$ bei einer Plazeboresponse-Rate von $24,1 \%$ [487]. In der MEASURE 3-Studie zeigte sich für die 150-mg (bzw. 300-mg)-Dosierung eine ähnliche ASAS-20-Antwort von 58,1\% (bzw. 60,5\%) bei einer Plazeboresponse-Rate von 36,8\% [488]. In allen Zulassungsstudien konnten somit eine Reduktion der Wirbelsäulenschmerzen und der Morgensteifigkeit sowie eine Verbesserung der Funktionsfähigkeit erreicht werden.

Die Wirksamkeit von Secukinumab auf das Vorhandensein extraspinaler Manifestationen ist nicht in gesonderten RCTs untersucht worden. Secukinumab hat eine exzellente Wirksamkeit in Bezug auf eine Psoriasis vulgaris, aber keine Wirksamkeit in Bezug auf eine akute anteriore Uveitis oder eine CED. In den Zulassungsstudien konnte gezeigt werden, dass die Lebensqualität unter einer Therapie mit Secukinumab signifikant gegenüber Plazebo ansteigt $[489,490]$. In der Proof-of-concept-Studie mit Secukinumab konnte gezeigt werden, dass es zu einem mittels MRT nachweisbaren Rückgang der Entzündung in der Wirbelsäule kommt [491].

Secukinumab ist mit einer Dosis von $150 \mathrm{mg}$ s.c. alle 4 Wochen im Anschluss an eine wöchentliche Aufdosierung über die ersten 4 Wochen mit $150 \mathrm{mg}$ s.c. für die AS zugelassen. Es liegen auch Daten aus Phase-III-Studien zur Dosierung mit einer i.v.-Aufdosierung (Secukinumab i.v. $10 \mathrm{mg} / \mathrm{kgKG}$ in Woche 0,2 und 4 ), mit nachfolgender monatlicher Dosierung wie bei den s.c.-Gaben, zu einer Dosierung mit $75 \mathrm{mg}$ (statt $150 \mathrm{mg}$ ), aber auch mit Gaben von $300 \mathrm{mg}$ s.c. alle 4 Wochen (wie sie bei der PsA zugelassen ist) vor.

Inzwischen liegen für die MEASURE 1- und 2-Studie 3-Jahres-Daten vor, die eine lang anhaltende Wirksamkeit 
der Therapie mit Secukinumab zeigen [492-494].

8.4.2.3 Andere Biologika. WeiterebDMARDs sind bei Patienten mit AS zwar untersucht worden, haben sich jedoch als ineffektiv erwiesen.

In einer prospektiven offenen PhaseII-Studie mit Rituximab bei AS-Patienten mit und ohne Versagen auf eine vorangegangene Therapie mit TNFi zeigte sich ein besseres Ansprechen in der Gruppe der TNFi-naiven Patienten [495]. Die ASAS20-Ansprechrate lag in dieser Gruppe bei $50 \%$. Jedoch fehlte in dieser Studie eine Kontrollgruppe.

Eine weitere prospektive offene Studie mit Abatacept bei Patienten mit und ohne Versagen auf eine vorangegangene Therapie mit TNFi zeigte kein Ansprechen [496]. Die ASAS-20-Ansprechrate lag zwischen 20 und $27 \%$ - und ist damit deutlich niedriger, als bei den TNFi berichtet.

Kontrollierte, randomisierte Studien mit Tocilizumab bzw. Sarilumab erreichten nicht den primären Endpunkt, sodass keine Interleukin-6 blockierende Substanz bei Patienten mit axSpA zur Verfügung steht [497, 498].

Ustekinumab hat sich sowohl in einer Proof-of-concept-Studie als auch in Phase-II-Studien als unwirksam in der Therapie der AS erwiesen [499, 500].

\subsubsection{Einleitung einer Therapie mit} bDMARDs. Die Einleitung einer bDMARD Therapie sollte gemäß internatioanler Empfehlungen erfolgen.

8.4.2.4.1 Internationale Empfehlungen. Für die Einleitung einer Therapie mit Biologika liegen evidenzbasierte Empfehlungen von ASAS und EULAR vor (• Abb. 4 und 5; [21]). Entsprechend dieser Empfehlung soll die Effektivität der Therapie nach 12 Wochen überprüft werden. Bei Patienten, die ein Ansprechen zeigen (BASDAI-Verbesserung um $\geq 2$ Punkte [auf einer Skala von 0-10] oder eine Verbesserung im ASDAS um $\geq 1,1$ ) und bei denen eine positive Expertenmeinung für eine Fortführung vorliegt, kann die Therapie fortgeführt werden.

Zusammengefasste Empfehlungen dieser S3-Leitlinie zur bDMARD-Therapie:

\subsection{Retentionsrate der bDMARD-}

Therapie. Die 1-Jahres-Retentionsrate in einem norwegischen Register für TNFi lag bei Patienten mit AS bei 77,5\% und ist damit im Vergleich zu anderen rheumatischen Erkrankungen (RA 65,4\%, Psoriasisarthritis 77,3\%) am höchsten [501]. Die 2-Jahres-Retentionsrate lag in einem schwedischen Register bei $74 \%$ [432]. In der dänischen Kohorte betrug die mittlere Dauer der Medikation 3,1 Jahre (erster TNFi) bzw. 1,6 (zweiter TNFi) und 1,8 (dritter TNFi) Jahre $(p<0,001)$ [502]. Die Ansprechraten liegen hierbei ähnlich hoch, fallen in verschiedenen Populationen aber durchaus unterschiedlich aus. Etwa 50-80\% der Patienten mit axialer SpA erreichen eine Ansprechrate mit einer ASAS-20-Antwort, 30-60\% ASAS40-Antwort, und 20-50\% kommen sogar in Remission. In kontrollierten Studien mit Populationen, die eine kurze Erkrankungsdauer (hier <3 Jahre) aufwiesen, konnte eine höhere Rate der (partiellen) Remission erreicht werden (z. B. INFAST mit 61,9\%) [381].

Der stärkste Prädiktor für eine dauerhafte Remission im Jahr 1 und 5 nach Einleitung der TNFi-Therapie war das Erreichen einer Remission in Woche 12 nach Beginn einer TNFi-Therapie [324]. Prädiktoren für ein schlechtes Ansprechen auf eine TNFi-Therapie sind Nikotinkonsum sowie Übergewicht [503-505]. Adipöse Patienten hatten eine niedrige Wahrscheinlichkeit, ein ASAS-40-Ansprechen zu erreichen, als normalgewichtige Patienten (OR 0,27 [95\%-CI $0,09-0,70]$ vs. 0,62 [95\%-CI 0,24-1,14]) [505].

Bei Patienten mit AS und vorwiegend axialer Beteiligung werden die TNFi als Monotherapie verwendet, da sich kein zusätzlicher Nutzen einer begleitenden Basistherapie mit Methotrexat zeigen ließ (s. Abschn. 8.4.3.2) [506-508]. Es liegen allerdings nur Daten zu einer Kombinationstherapie mit Infliximab und Methotrexat vor. In einer Studie mit schlechter Studienqualität wurde über einen zusätz- lichen Effekt von MTX in Kombination mit Infliximab berichtet. Grund für die Nichtbewertung dieser Aussage ist die geringe Patientenanzahl (19 AS-Patienten), die fehlende Verblindung und das hohe Risiko einer Verzerrung der Studienergebnisse (Bias) [509]. 8.4.2.4.3 Stratifikation IL-17-Inhibito-
ren und TNF-Inhibitoren untereinander. Es liegen weder Vergleichsstudien (Head-to-head-Studie) zwischen TNFi und IL-17-Inhibitoren noch Strategiestudien bei Patienten mit axialer SpA vor. Aufgrund der unterschiedlichen Wirksamkeit von TNFi und Secukinumab in Bezug auf extraskeletale Manifestationen kann in der klinischen Entscheidungsfindung die Wirksamkeit auf eine begleitende Psoriasis oder AAU bzw. CED einer Einzelsubstanz berücksichtigt werden. Zudem sind die Unterschiede im Zulassungsstatus (TNFi für axSpA-Gesamtgruppe, Secukinumab für AS) $\mathrm{zu}$ berücksichtigen.

8.4.2.5 Unerwünschte Wirkungen einer bDMARD-Therapie. Die unerwünschten Wirkungen einer Therapie mit Biologika sind potenziell vielfältig, das NutzenRisiko-Profil wird jedoch allgemein als günstig eingeschätzt. Die Datenlage bezüglich unerwünschter Wirkungen ist für TNFi sehr viel größer als für Secukinumab, für das bisher nur Sicherheitsdaten aus den kontrollierten Studien zur Verfügung stehen.

Die Inzidenz unerwünschter Ereignisse der Therapie mit TNFi wird mit einem Risiko von $\mathrm{RR}=1,22$; $95 \%$-CI: 1,12-1,33 angegeben [510]. Da die meisten Biologika s.c. appliziert werden, sind Probleme an der Injektionsstelle beschreiben worden, stellen in der klinischen Versorgung jedoch kein relevantes Problem dar. In einer Metaanalyse wurde die Inzidenz einer Reaktion an der Injektionsstelle mit einem Risiko von $\mathrm{RR}=2,93$; $95 \%$-CI: 2,02-4,23 angegeben [510]. In der täglichen Praxis konzentriert sich die Aufmerksamkeit hinsichtlich der unerwünschten Wirkungen vor allem auf die gesteigerte Rate an Infektionen inklusive der Reaktivierung einer latenten Tuberkulose (TB) und einer infektiösen Hepatitis, insbesondere der Hepatitis B. Das ef- 
fizienteste Vorgehen zur Minderung des Risikos für TB ist vor Einleitung einer Biologikatherapie ein sorgfältiges Screening auf eine latente TB-Infektion und ggf. umgehende Einleitung einer TB-Prophylaxe [511]. In den verfügbaren Metaanalysen zeigt sich eine nicht signifikant erhöhte Rate schwerwiegender Infektionen bei Patienten mit AS unter Biologika [512, 513]. In der Metaanalyse aus dem Jahr 2010 wurden schwerwiegende Infektionen in der Plazebogruppe mit einer Häufigkeit von 0,4/100 Patientenjahren beschrieben im Vergleich zur Verumgruppe mit 1,9/100 Patientenjahren. Eine weitere Metaanalyse zeigte keinen signifikanten Unterschied in der Inzidenz schwerwiegender unerwünschter Ereignisse zwischen den verschiedenen TNFi [510]. In der Metaanalyse aus dem Jahr 2017 wurde bestätigt, dass schwere Infektionen bei Patienten mit AS unter TNFi nicht signifikant häufiger im Vergleich zu plazebobehandelten Patienten auftreten (RR 1,57 [95\%-CI 0,63-3,91]) [513]. In dieser Metaanalyse zeigte sich, dass bei Durchführung von einem Screening auf Tuberkulose kein erhöhtes Risiko unter einer Therapie mit TNFi besteht (RR 2,52 [95\%-CI, 0,53-12,09]).

Selten kann es unter einer Therapie mit einem TNFi zu malignen Erkrankungen kommen. In einer Metaanalyse aller TNFi und Einbeziehung von Patienten mit diversen entzündlich rheumatischen Erkrankungen lag das zusammengefasste Risiko bei 0,95 (95\%-CI 0,85-1,05) [514]. In dieser Metaanalyse zeigte sich ein erhöhtes Risiko für die Entwicklung maligner Hauttumoren, insbesondere von Nicht-Melanomen (1,45 [95\%-CI 1,15-1,76]). In einzelnen Fällen wurde über kardiale Dekompensationen (Herzinsuffizienz Grad III und IV sind Kontraindikationen) und entzündliche neuromuskuläre Erkrankungen berichtet.

Die Sicherheitsdaten aus den Secukinumab-Studien zeigen ebenfalls ein erhöhtes Risiko für die Entwicklung von Infektionen. Allerdings fehlen Realworld-Daten sowie Longitudinaldaten über einen längeren Zeitraum.

8.4.2.6 Dosisreduktion bzw. Absetzen der bDMARD-Therapie. Ein Absetzen der Therapie führt bei einem hohen Prozentsatz der AS-Patienten zu klinischen Rückfällen, diese treten zum Teil bereits nach 7 Wochen auf, die mittlere Zeit bis zum Rückfall beträgt 17 Wochen [515]. Eine Wiederaufnahme der bDMARDTherapie scheint aber ohne wesentliche Probleme möglich zu sein, und die meisten, aber nicht alle Patienten sprechen auch erneut an. Die erneute Zunahme der Krankheitsaktivität nach dem kompletten Absetzen der TNFi-Therapie ist konsistent in etablierten AS-Kohorten mit eher langer Erkrankungsdauer gezeigt worden [515].

Dosisreduktion ist möglicherweise eine Option für Patienten, die unter einer TNFi-Therapie eine Remission bzw. niedrige Krankheitsaktivität erreicht haben. ${ }^{3}$ In einer kontrollierten Studie mit Infliximab bei Patienten mit axialer SpA und kurzer Erkrankungsdauer konnte gezeigt werden, dass bei Patienten in Remission unter einer Infliximab-Therapie der TNFi abgesetzt werden konnte und ca. 50\% der Patienten innerhalb der folgenden 28 Wochen in Remission verbleiben [517]. Retrospektive Studien konnten zeigen, dass ca. $60 \%$ der ASPatienten mit niedriger Krankheitsaktivität die bDMARD-Dosis um ein Drittel reduzieren konnten [518]. In einer französischen Studie zeigte sich, dass die kontinuierliche Gabe mit $5 \mathrm{mg} / \mathrm{kgKG}$ Infliximab alle 6 Wochen in einem festen Schema der Infusion mit $5 \mathrm{mg} / \mathrm{kgKG}$ Infliximab bei Bedarf durchgeführten Gabe überlegen ist [506]. Eine niedrig dosierte Gabe mit Infliximab $3 \mathrm{mg} / \mathrm{kgKG}$ ist Plazebo ebenfalls überlegen (ASAS20 -Antwort $53,5 \%$ vs. $30,6 \%, p=0,042$ ) [519]. Jedoch haben $68 \%$ der Patienten in der offenen Beobachtungsphase eine Dosiseskalation auf Infliximab $5 \mathrm{mg} /$ kgKG benötigt, um die Symptome der AS adäquat $\mathrm{zu}$ behandeln.

\footnotetext{
${ }^{3}$ Nach Fertigstellung der SLR wurde eine kontrollierte Studie bei Patienten mit nr-axSpA publiziert, in der gezeigt wurde, dass bei Patienten in klinischer Remission unter einer Therapie mit Adalimumab das Absetzen der bDMARD-Therapie zwar zu einer höheren Rate an Schüben führte, aber dass trotzdem $47 \%$ der Patienten in dem Beobachtungszeitraum keinen Schub erlitten [516].
}

8.4.2.7 Wirkverlust und Switching der bDMARD-Therapie. bDMARDs können neben einem primären Wirkverlust (eine Wirksamkeit kann von Anfang an nicht beobachtet werden) auch einen sekundären Wirkverlust zeigen, d. h. nach einer Phase der Wirksamkeit tritt ein Wirkverlust ein. Die Genese wird kontrovers diskutiert, und die Ursache ist bislang unklar, wobei immunogene Faktoren und die Entwicklung von Autoantikörpern diskutiert werden. Eine französische Studie zeigte, dass kein Zusammenhang zwischen den Infliximab-Serumkonzentrationen und der Krankheitsaktivität nach einer 1-jährigen Therapie mit Infliximab besteht [520]. Im Gegensatz zu dieser Studie hat eine niederländische Gruppe eine Korrelation zwischen der Höhe der Serumspiegelbestimmung verschiedener TNFi und der Krankheitsaktivität gefunden [521]. Es zeigte sich insbesondere eine negative Korrelation zwischen Höhe der Serumspiegelbestimmung und der Krankheitsaktivität für Adalimumab nach 6 Monaten Therapie. In dieser Studie wurden die Patienten auch auf die Induktion von Antikörpern gegen TNFi untersucht. Diese und eine weitere Studie aus den Niederlanden stützen die These, dass für den sekundären Wirkverlust bei Patienten mit AS unter Therapie mit einem TNFi, Autoantikörper gegen Adalimumab und Infliximab eine Rolle spielen können [521, 522]. In diesen Studien konnte keine Antikörperbildung gegen Etanercept nachgewiesen werden [523]. Die Untersuchung auf Antikörper gegen TNFi ist derzeit nicht kommerziell erhältlich.

Die Datenlage zu Therapiewechsel bei bDMARD-Therapie ist spärlich und bezieht sich überwiegend auf TNFiDaten. Der Wechsel von einem TNFi zu einem anderen ist möglich, ist aber mit einem schlechteren Therapieansprechen verknüpft. Diese Aussage basiert auf 3 systematischen Reviews und mehreren Registerstudien; kontrollierte Studien fehlen [502, 524-527]. Beide Reviews zeigen, dass der Wechsel für einen Teil 
der Patienten erfolgreich ist, aber mit einem schlechteren Therapieansprechen verknüpft ist. „Drug survival“ war bei dem zweiten TNFi (47-72\% über 2 Jahre) oder dritten TNFi (49\% über 2 Jahre) niedriger als beim ersten TNFi. In der dänischen Kohorte mussten $30 \%$ der Patienten auf einen zweiten TNFi umgestellt werden, wobei der Hauptgrund für die Umstellung der sekundäre Wirkverlust war [502]. Von den umgestellten Patienten erreichten immer noch $52 \%$ der Patienten eine klinische Remission, Daten der Schweizer Kohorte legen nahe, dass die mittlere Therapiedauer bei Patienten mit Wechsel auf einen zweiten TNFi bei primärer Wirkungslosigkeit deutlich kürzer ist als bei einem sekundären Wirkverlust (mittlere Therapiedauer mit einem zweiten TNFi: 1,06 Jahre [95\%-CI 0,75-1,96]) nach primären Versagen vs. 3,76 Jahre (95\%-CI 3,12-4,28) nach sekundärem Versagen [525]. In einer prospektiven longitudinalen Kohorte aus Schweden mit 514 AS-Patienten wechselten $77 \mathrm{~Pa}$ tienten auf einen zweiten TNFi entweder wegen Wirkverlust oder wegen Nebenwirkungen [524]. Die Krankheitsaktivität konnte zwar für einige Patienten gesenkt werden, die Krankheitsaktivität war aber höher als in der Patientengruppe, die keinen Wechsel der Medikation durchführen mussten.

Daten zur Effektivität einer Änderung des Wirkprinzips liegen nicht vor.

\section{Empfehlung/Statement}

\section{8-15}

Eine Therapie mit Biologika soll bei Patienten mit persistierend hoher entzündlicher Krankheitsaktivität und unzureichendem Ansprechen auf eine NSARTherapie oder Unverträglichkeit von NSAR begonnen werden. Dabei sind Unterschiede in der Zulassung für TNFund IL-17-Inhibitoren zu beachten. Empfehlungsgrad: A

Evidenz: 1++

\section{8-16}

Bei Patienten mit axialer SpA und symptomatischer peripherer Arthritis sollte eine TNF-Blocker-Therapie versucht werden, wenn der Patient auf mindestens eine lokale Steroidinjektion ungenügend angesprochen hat und ein angemessener Behandlungsversuch mit einem Basistherapeutikum, bevorzugt Sulfasalazin, keine Wirkung gezeigt hat. Empfehlungsgrad: $B$

Evidenz: 1

Kommentar zu 8-16:

Diese Empfehlung setzt sich aus Informationen von mehreren Studien zusammen. Sequenzielle Studien (lokales Steroid, Sulfasalazin-Therapie und danach Therapie mit einem TNFi) bei Patienten mit peripherer Arthritis sind nicht durchgeführt worden. Daher wird der Empfehlungsgrad von „A“ auf „B“ herabgestuft.

\section{8-17}

Bei Patienten mit extramuskuloskeletalen Manifestationen, insbesondere bei Vorliegen einer Uveitis, chronisch entzündlichen Darmerkrankung oder Psoriasis sollte die unterschiedliche Effektivität der verschiedenen Biologika auf diese Manifestationen beachtet werden.

Empfehlungsgrad: B

Evidenz: $1+/ 2 b$

\section{8-18}

Bei Patienten mit verbleibenden muskuloskeletalen Symptomen unter einer Biologikatherapie kann eine zusätzliche Therapie mit NSAR erfolgen. Empfehlungsgrad: Statement Evidenz: -

\section{8-19}

Die Wirksamkeit einer Biologikatherapie soll nach 12 Wochen überprüft werden.

Empfehlungsgrad: A

Evidenz: 1++

\section{8-20}

Bei Patienten, die ein Ansprechen zeigen (BASDAI-Verbesserung um $\geq 2$ Punkte [auf einer Skala von 0-10] oder eine Verbesserung im ASDAS um $\geq 1,1$ ) und bei denen eine positive Expertenmeinung für eine Fortführung vorliegt, kann die Therapie fortgeführt werden.

Bei Patienten ohne Ansprechen sollte ein Absetzen in Erwägung gezogen werden.

Empfehlungsgrad: $B$

Evidenz: $2 b$

\section{8-21}

Eine Empfehlung, ob mit einem TNFInhibitor oder mit einem IL-17-Inhibitor begonnen werden soll, kann aufgrund der Studiendaten zur Wirksamkeit auf das Achsenskelett und Sicherheit nicht gegeben werden. Für TNF-Inhibitoren bestehen längere Erfahrungen in der klinischen Anwendung. Empfehlungsgrad: Statement Evidenz: -

\section{8-22}

Bei nicht ausreichender Wirksamkeit eines Biologikums und bestehender hoher entzündlicher Krankheitsaktivität sollte der Wechsel auf ein weiteres Biologikum erfolgen. Empfehlungsgrad: B

Evidenz: 2

\section{8-23}

Bei Patienten in anhaltender Remission (mindestens für 6 Monate) unter einer Biologikagabe können eine Dosisreduktion bzw. eine Intervallverlängerung und später evtl. auch das Absetzen des Biologikums erwogen werden. Empfehlungsgrad: B

Evidenz: 2

\subsubsection{Chemisch-synthetische} "disease-modifying antirheumatic drugs" (csDMARDs) (sog. Basistherapie)

Der Begriff „Basistherapeutika“ ist bei Patienten mit axialer SpA irreführend, da im Gegensatz zur RA diese Therapieoption nicht als „Basis“ der Therapie angesehen wird. Da es sich aber in der 
Rheumatologie um einen weitverbreiteten Begriff handelt, wird der Begriff „Basistherapie bzw. Basistherapeutikum " in dieser Leitlinie weiterverwendet. In der Literatur wird kontrovers diskutiert, ob die Komedikation mit einem DMARD zusätzlich zu einer bestehenden Biologikatherapie die Zeitdauer der Biologikagabe verlängert. In einer portugiesischen Kohorte mit 954 Patienten hatte die Gabe von DMARDs keinen messbaren Effekt aufdie Retention derTNFiüber die Zeit [528], wohingegen die Kollegen der Schweizer Kohorte zeigten, dass eine TNFi-Monotherapie zu einer geringeren Retention der Biologikamedikation führt (HR 1,17, 95\%-CI 1,01-1,35) [529].

8.4.3.1 Sulfasalazin. Es liegt ein Cochrane-Review mit Update von 2014 zum Gebrauch von Sulfasalazin bei Patienten mit AS vor [530, 531]. Nach Auswertung von 12 randomisierten kontrollierten Studien kommen die Autoren zu dem Schluss, dass Sulfasalazin bei axialer Manifestation keinen klinisch relevanten Einfluss auf die Wirbelsäulensymptome hat, dass aber Sulfasalazin möglicherweise eine Rolle in der Behandlung der aktiven peripheren Arthritis spielt. In einer Langzeitstudie über 3 Jahre traten unter Sulfasalazin weniger Episoden von peripheren Gelenkbeschwerden auf als bei den mit Plazebo behandelten Patienten. In den im Review eingeschlossenen Studien, bei denen sich Hinweise auf eine Wirksamkeit auf periphere Symptome ergaben, lag eine kurze Krankheitsdauer mit einer starken entzündlichen Komponente vor. In einer norwegischen Kohortenstudie zeigte sich, dass Patienten mit peripherer Arthritis stärker von einer Sulfasalazin-Therapie profitierten als Patienten mit reiner Achsenskelettbeteiligung [532]. Eine kleine kontrollierte Studie mit RA- und AS-Patienten zeigte bei Beobachtung über 1 Jahr keine Wirksamkeit von Sulfasalazin bei Patienten mit Enthesitis [533]. Die Wirksamkeit von Sulfasalazin scheint sich zwischen Patienten mit kurzer und langer Krankheitsdauer $\mathrm{zu}$ unterscheiden. In einer Studie zeigte sich, dass Patienten mit kurzer Erkrankungsdauer ( $<5$ Jahre) auf eine Therapie mit Sulfasalazin auch bei axialen Symptomen mit allerdings geringer Ef- fektstärke ansprechen [534]. Die Wirksamkeit von Sulfasalazin bei Patienten mit kurzer Erkrankungsdauer und jungem Erkrankungsalter (<25 Jahre) konnte in einer 2017 publizierten randomisierten Studie bestätigt werden [535]. In dieser kleinen Studie erreichte allerdings kein Patient eine ASDAS-Remission. In 2 weiteren Studien wurde Sulfasalazin mit Etanercept bei Patienten mit aktiver AS bzw. mit nr-axSpA-Patienten verglichen [426, 447]. Da die Studien insgesamt wegen der Verwendung verschiedener Outcome-Parameter nur schwer vergleichbar sind und die Qualität der Studien ebenfalls variiert, sind generelle Aussagen hierzu schwierig. In der Studie mit AS-Patienten erreichte die Etanercept-Gruppe zwar signifikant häufiger den primären Endpunkt ASAS-20Ansprechrate in Woche 16 als die mit Sulfasalazin behandelte Gruppe (ASAS-20Ansprechrate: $76 \%$ vs. $53 \%$; $p<0,0001$ ), die Ansprechrate war in der SulfasalazinGruppe jedoch nach 16 Wochen Therapie relativ hoch $(53 \% ; p<0,0001)$ [447]. In der zweiten genannten Studie (Einschlusskriterium: Patienten mit axialer SpA und einem Krankheitsverlauf von weniger als 5 Jahren [AS-Patienten und Patienten mit nr-axSpA]) zeigte sich bezogen auf den primären Endpunkt (Veränderung entzündlicher Areale in der MRT in Woche 48) kein Unterschied in der Etanercept- und in der Sulfasalazin-Gruppe. Nach 48 Wochen zeigte sich bei $50 \%$ der mit Etanercept und $19 \%$ der mit Sulfasalazin behandelten nraxSpA-Patienten eine klinische Remission [426]. Eine neuere Metaanalyse aus China vergleicht die Wirksamkeit von mit Sulfasalazin behandelten Patienten im Vergleich zu mit Etanercept behandelten Patienten [536]. Auch in dieser Studie wurde die Überlegenheit von Etanercept auf sämtliche Outcome-Variablen bestätigt. Eine Kombinationstherapie Etanercept plus Sulfasalazin wurde in keiner Studie durchgeführt. Eine chinesische Studie untersuchte, ob sich nach einer durch Biologika induzierten klinischen Remission die Remission durch die Gabe von Sulfasalazin im Vergleich zu Thalidomid bzw. NSAR aufrechterhalten ließ [537]. In allen 3 Armen zeigte sich eine Rezidivrate von deutlich mehr als $50 \%$ (NSAR $89,2 \%$, SSZ $84,4 \%$, Thalidomid 60\%).

Darüber hinaus wurde gezeigt, dass eine Sulfasalazin-Therapie das Auftreten von akuten Episoden einer anterioren Uveitis bei AS-Patienten verhindert [88]. Patienten mit einer Colitis ulcerosa oder Crohn-Kolitis mit leichter bis mäßiger Aktivität sollten entsprechend der DGVS-Leitlinie entweder mit Sulfasalazin oder systemisch wirksamen Glukokortikoiden behandelt werden [104].

8.4.3.2 Methotrexat. Methotrexat (MTX) hat auf die Wirbelsäulensymptomatik von AS-Patienten keine Wirkung [538, 539]. Ob ein Effekt auf die periphere Gelenksymptomatik von Patienten mit AS vorliegt, ist nicht klar, da keine der 3 randomisierten kontrollierten Studien eine ausreichende Fallzahl aufweist [538]. Die aktuellen Studien untersuchen alle die Wirkung von MTX in Kombination mit TNFi [506, 508, 509]. In 2 randomisierten kontrollierten Studien zeigte sich keine zusätzliche Wirksamkeit (gemessen mit ASAS-20-Kriterien) von MTX gegenüber Infliximab allein [506, 508]. In einer kleinen Studie $(n=19)$ im Parallelgruppendesign zeigte sich eine bessere Wirksamkeit von MTX in Kombination mit Infliximab verglichen mit Infliximab alleine nach 30 Wochen (gemessen mit BASDAI 50) [509]. Da es sich hierbei aber um eine recht kleine Studie mit hohem Risiko für Bias der Studienergebnisse handelt, wird das Ergebnis kritisch bewertet.

\subsubsection{Andere medikamentöse Therapien}

Insgesamt gibt es keine überzeugende Evidenz, um den Gebrauch von anderen traditionellen DMARDs bei Patienten mit AS zu empfehlen - dies betrifft vor allem die Achsenskelettsymptomatik und schließt Gold, Hydroxychloroquin, D-Penicillamin, Ciclosporin A und Leflunomid ein [418].

Glukokortikoide: Es gibt lediglich eine kleine Studie $(n=39$, Laufzeit 2 Wochen) zu oralen Glukokortikoiden (sog. systemische Applikation), die die Wirksamkeit von Prednisolon $50 \mathrm{mg} / \mathrm{Tag}$ vs. $20 \mathrm{mg} / \mathrm{Tag}$ vs. Plazebo bei Patienten mit 
AS randomisiert-kontrolliert untersucht hat $[418,540]$. Der primäre Endpunkt BASDAI 50 wurde von 33 und $27 \%$ in der 50- und 20-mg-Dosierung, aber nur von $8 \%$ der Plazebogruppe erreicht. Die mittlere BASDAI-Verbesserung war in der 50-mg-Dosierung signifikant größer ausgeprägt als in der 20-mg-Dosierung $(2,39 \pm 0,5$ vs. $1,19 \pm 0,53 ; p=0,410$; Plazebo $0,66 \pm 0,49)$. Die Studie zeigt, dass orale Glukokortikoidgaben in höheren Dosierungen wirksam sind, und die erreichten Verbesserungen müssen vor dem Hintergrund des Potenzials einer bDMARD-Versorgung gesehen werden.

Nur wenige Studien befassen sich mit der lokalen Applikation von Glukokortikoiden - entweder als intraartikuläre Injektion oder als Injektion im Bereich der Enthesen. Die Datenlage wird im Abschn. 8.6.1 erläutert.

Siehe auch Empfehlung 8-28 und 8-29 zur lokalen Injektion von Glukokortikoiden (Abschn. 8.6.1).

Intravenöse Bisphosphonate: Bisphosphonate weisen keinen krankheitsmodifizierenden Effekt bei Patienten mit AS auf [541-546]. In der randomisierten kontrollierten Studie aus Kanada wurde zwar mit der höheren Dosis Pamidronat eine signifikant bessere Wirkung sowohl für Wirbelsäulenschmerz als auch für Funktion ermittelt, jedoch erst beginnend ab Monat 4. Da die Behandlung mit Pamidronat mit transienten Gelenkschmerzen und Muskelschmerzen nach der Infusion bei der Mehrheit der Patienten assoziiert ist, ist eine prospektive doppelblinde kontrollierte Studie kaum möglich. Studien, die die Wirksamkeit von Bisphosphonaten auf Patienten mit AS und Osteoporose untersuchen, liegen nicht vor.

Komplementärmedizin: Patienten mit chronischen Erkrankungen nutzen komplementärmedizinische Angebote. In einer Querschnittstudie aus Australien gaben $94,7 \%$ an, dass sie komplementärmedizinische Angebote genutzt haben [547]. Zwei randomisierte kontrollierte Studien überprüften die Wirksamkeit von Probiotika bei Patienten mit SpA [548, 549]. Beide Studien zeigen keinen Unterschied gegenüber Plazebo in Bezug auf Krankheitsaktivität, Funktionsfähigkeit und körperliches Wohlbefinden.

\section{Empfehlung/Statement}

\section{8-24}

Bei Patienten mit axialer SpA und klinisch führender peripherer Arthritis sollte eine Basistherapie mit Sulfasalazin durchgeführt werden (B). Andere Basistherapeutika wie Methotrexat können alternativ eingesetzt werden (Expertenkonsens). Empfehlungsgrad: $B$

Evidenz: 1

Kommentar zu 8-24:

Diese Empfehlung basiert auf einer Cochrane-Analyse, die einen geringen Effekt der Sulfasalazin-Behandlung bei Patienten mit peripherer Arthritis diskutiert hat. Daher wird der Empfehlungsgrad von „A“ auf „B“ herabgestuft.

\section{8-25}

Bei Patienten mit AS sollte keine Behandlung der Wirbelsäulensymptomatik mit Methotrexat erfolgen. Empfehlungsgrad: $B$

Evidenz: 1

Kommentar zu 8-25:

Herabstufung des Empfehlungsgrads von „A“ auf „B", da hier eine Extrapolation der Ergebnisse aus der Evidenzebene 1 vorgenommen wurde.

\section{8-26}

Es gibt keine ausreichende Evidenz, eine Kombination von TNF-Inhibitoren mit MTX zur Vermeidung von „antidrug-antibodies" (ADAs) zu empfehlen. Empfehlungsgrad: Statement Evidenz:

\section{8-27}

Die systemische Langzeitgabe von Glukokortikoiden wird bei Patienten mit Achsenskelettbeteiligung nicht empfohlen. Für die Wirksamkeit einer kurzfristigen Therapie mit Glukokortikoiden gibt es nur sehr begrenzte Evidenz. Empfehlungsgrad: 0

Evidenz: 4

\subsection{Familienplanung vor dem Hintergrund einer medikamentösen Therapie}

\section{Schlüsselfrage 9}

- Welche Aspekte müssen bei Patienten/Patientinnen mit Kinderwunsch bzw. in der Schwangerschaft beachtet werden?

Familienplanung: Bei der Frage nach der Wahl der Therapiestrategie sowie der Überwachung der eingesetzten Therapie klingen auch immer häufiger Fragen zu Kinderwunsch, Schwangerschaft und Stillzeit an. Sowohl die EULAR als auch die britischen Kollegen haben hierzu Stellungnahmen publiziert [550, 551]. In beiden Stellungnahmen wird zu dem Einsatz von csDMARD und bDMARD bei Frauen mit Kinderwunsch bzw. bestehender Schwangerschaft detailliert Stellung genommen.

Der Einfluss von TNFi auf die Spermatogenese wird kontrovers diskutiert. Es gibt Hinweise, dass sich die bei $\mathrm{Pa}$ tienten mit einer aktiven AS bestehenden Abnormalitäten der Spermien unter einer Therapie mit TNFi zurückbilden [552].

In Registern ist der Verlauf von einigen Hundert Schwangerschaften dokumentiert, allerdings überwiegend bei Patienten mit RA [553]. In einer schwedischen Fall-Kontroll-Studie zeigte sich, dass $\mathrm{Pa}$ tientinnen mit AS eine höhere Prävalenz an Schwangerschafts- und Geburtskomplikationen hatten, die die Autoren sowohl auf die Krankheitsschwere als auch bestehende Komorbiditäten zurückführten [554]. Sowohl die Rate an Notfallund elektiven Kaiserschnitten war erhöht (OR 3,00 [95\%-CI 2,01-4,46] bzw. 1,66 [95\%-CI 1,09-2,54]) als auch die Rate an Frühgeburten und niedrigem Geburtsgewicht (OR 1,92 [95\%-CI 1,17-3,15] bzw. 2,12 [95\%-CI 1,00-4,50]). In einer prospektiven Schweizer Kohorte mit RAund axSpA- $(n=62)$ Patienten zeigte sich, dass knapp ein Drittel der Patientinnen einen Schub ihrer Grunderkrankung in der Schwangerschaft erlebte, wobei das relative Risiko bei 3,08 (95\%-CI 1,2-7,9) für die Entwicklung eines Schubes lag, wenn der TNFi mit Bekanntwerden der Schwangerschaft pausiert wurde [555]. 
In einer amerikanischen Kohorte konnte kein Zusammenhang zwischen Einnahme von oralen Kontrazeptiva und dem Beginn bzw. der Schwere der SpA festgestellt werden [556].

\subsection{Invasive Therapie}

\subsubsection{Injektionen}

\section{Schlüsselfrage 21}

- Bei welchen Patienten mit SpA kommt eine Injektionstherapie am Achsenskelett infrage?

Intraartikuläre Gelenkinjektionen können bei Patienten mit axialer SpA im Bereich der SI-Gelenke und im Bereich der peripheren Gelenke bzw. Enthesen durchgeführt werden. Kontrollierte Studien für Patienten mit $\mathrm{SpA}$ gibt es für die Injektion in die SI-Gelenke und in die Achillessehne [557-559]. Es fehlen kontrollierte Studien zur Injektion peripherer Gelenke und zu Injektionen im Bereich der Wirbelsäule.

Achillessehne: Es liegt nur eine kontrollierte Studie mit 12 AS-Patienten und schwergradiger unilateraler Enthesitis der Achillessehne vor, in der eine Kortikoidinjektion mit einer lokalen Etanercept-Injektion verglichen wird [557]. Die Injektion mit Betamethason war genauso effektiv wie die lokale Etanercept-Injektion, gemessen mit ASAS-20 in Woche 2, 4, 8 und 12.

SI-Gelenke: In einer kontrollierten Studie aus China wurde die Wirkung einer Radiofrequenzdenervierung mit der von Celebrex $400 \mathrm{mg} / \mathrm{Tag}$ verglichen, wobei sich ein stärkerer Effekt durch die Radiofrequenztherapie als durch die NSAR-Gabe nach 24 Wochen zeigte [560]. Eine kleine randomisierte, kontrollierte und doppelblinde Studie mit insgesamt 10 Patienten und symptomatischer florider Sakroiliitis (8 AS, 2 Patienten mit SAPHO-Syndrom) zeigt eine Verbesserung der Schmerzen im SI-Gelenk über 6 Monate für die Kortikosteroidgruppe im Vergleich zu der Plazebogruppe (Wirkstärke 1,94 [95\%CI 0,53-3,35]) [558]. Eine ältere Querschnittstudie mit 30 SpA-Patienten (9 AS, 11 uSpA-Patienten) zeigte ebenfalls eine Verbesserung der Schmerzen im SI-
Gelenk über 6 Monate [561]. Periartikuläre Injektionen im Bereich der SIGelenke zeigen ebenfalls eine Effektivität über 2 Monate [559]. Es gibt keine Vergleichsstudien zwischen CT-gesteuerter intraartikulärer SI-Gelenkinjektion und einer periartikulären Injektion.

\subsubsection{Totalendoprothese}

\section{Schlüsselfrage 19}

- Welche Indikation besteht zur Durchführung einer TEP?

Eine Beteiligung der Hüftgelenke geben bis zu 36\% der Patienten mit AS in Registerstudien an [562]. In den Registerdaten ist die Diagnose „Hüftbeteiligung“ aufgrund klinischer Kriterien gestellt worden und dann ins Register aufgenommen worden. Dies bedeutet, dass es sich um eine Koxitis gehandelt haben kann, eine enthesitische Beteiligung periartikulärer Strukturen ist jedoch auch möglich. Eine Totalendoprothese (TEP) der Hüfte wurde bei 5-8\% der Patienten in der gesamten Kohorte implantiert, bei $47 \%$ dieser Patienten wurde diese beidseitig vorgenommen. Patienten mit einer Erkrankungsdauer von über 30 Jahren haben in $12-25 \%$ eine Hüft-TEP erhalten. Patienten mit einer Hüftgelenkbeteiligung hatten eine signifikant schlechtere körperliche Funktionsfähigkeit. Das Risiko für eine Hüft-TEP-Implantation war für AS-Patienten in einer taiwanesischen Fall-Kontroll-Studie deutlich gegenüber einer gesunden Vergleichspopulation erhöht (Inzidenzrate: 12,59 [95\%CI 5,54-28,58]) [563].

Nach einem Hüftgelenkersatz berichten $83 \%$ der Patienten über eine gute bis sehr gute Schmerzreduktion, und $52 \%$ der Patienten berichten über eine gute bis sehr gute Funktionsfähigkeit [418]. Es liegen Daten zu 340 AS-Patienten mit Hüft-TEP vor, die im Mittel über 14 Jahre nachverfolgt wurden. Verglichen mit Patienten, die aus anderen Gründen eine Hüft-TEP erhalten haben, sind die AS-Patienten deutlich jünger - im Mittel 40 Jahre alt [418]. Revisionen werden entsprechend den Daten aus England überwiegend in den ersten 7 Jahren meist aufgrund einer Prothesenlockerung durchgeführt. Nach 10 Jahren mussten $90 \%$ der TEP und nach 20 Jahren $65 \%$ der TEP nicht gewechselt werden [418]. Die in den initialen Berichten gesehene hohe Rate an heterotopen Ossifikationen (um $80 \%)$ bestätigt sich in jüngeren Publikationen nicht (22\%) [564]. Die Patienten erhalten in den Fallserien, die in jüngerer Zeit publiziert wurden, eine kontinuierliche NSAR-Therapie perioperativ zur Verhinderung heterotoper Ossifikationen. Kontrollierte Studien liegen hierzu nicht vor.

\subsubsection{Wirbelsäulenoperation}

\section{Schlüsselfrage 20}

- Wann sollte eine Korrektur-OP der

Wirbelsäule empfohlen werden?

Wirbelsäulenkorrekturosteotomie: ASPatienten, die aufgrund ihrer Wirbelsäulendeformität die Fähigkeit zur horizontalen Sicht verloren haben, kommen für eine Aufrichtungsoperation mit Korrekturosteotomie infrage $[418,565,566]$. Es kann keine der 3 Techniken (Opening-wedge-Osteotomie, Polysegmental-wedge-Osteotomie, Closing-wedgeOsteotomie $=$,pedicle subtraction osteotomy“ [PSO]) eindeutig favorisiert werden [418]. Eine Winkelverbesserung der Wirbelsäule zwischen 10 und $60^{\circ}$ kann erzielt werden [418, 567]. Die Komplikationsrate schwankt zwischen den Studien beträchtlich. Neben meist temporären neurologischen Störungen handelt es sich dabei vor allem um Implantatlockerungen mit konsekutivem Korrekturverlust und schmerzhafter Pseudarthrosenbildung.

Wirbelsäulenfrakturen: Aufgrund der verminderten Knochendichte und der fehlenden Pufferwirkung verknöcherter Bandscheiben und Gelenke (s. Abschn. 3.1.1.4) können AS-Patienten bereits ohne erinnerliches Trauma spontan eine Wirbelkörperfraktur erleiden. Wirbelsäulenverletzungen nach adäquatem Trauma sind am häufigsten an der HWS und am zweithäufigsten an der unteren BWS lokalisiert. Von den an der Halswirbelsäule Verletzten waren bei $67,2 \%$ der Patienten bei Einlieferung in das Krankenhaus bereits Lähmungen aufgetreten [53]. Wegen der Einsteifung der Bewegungssegmente frakturiert bei 
Patienten mit AS fast immer auch die hintere Säule (Wirbelbögen). Diese Verletzungen sind hoch instabil, lassen sich durch konservative Maßnahmen meist nicht suffizient ruhigstellen und sollten operativ angegangen werden [568-572]. Von diesen Autoren werden in kleinen Patientenkollektiven eine sehr hohe Komplikationsrate von $51,1 \%$ und eine Gesamtmortalitätsrate nach 3 Monaten von $17,7 \%$ nach der operativen Frakturversorgung angegeben. In einer Analyse aus den USA, die auf Krankenhausdaten basiert, zeigte sich eine erhöhte Sterblichkeit bei hospitalisierten AS-Patienten mit Halswirbelsäulenfraktur (OR 1,61 [95\%-CI 1,16-2,22]) (s. Abschn. 3.2.3) [161].

Die Bildgebung zur Erkennung einer Wirbelsäulenfraktur bei Patienten mit AS ist nicht standardisiert. Die Schnittbildverfahren MRT und CT liefern unterschiedliche Informationen. Eine angemessene Einschätzung ist in ausgewählten Fällen manchmal nur durch beide Verfahren gewährleistet. AS-Patienten mit einer akuten Wirbelfraktur sollten in einem spezialisierten operativen Wirbelsäulenzentrum vorgestellt werden.

Die Empfehlung 3-2 wurde aus dem Abschn. 3.1.1.4 hier eingefügt, da der Inhalt sich mit dem der invasiven Therapie überschneidet.

Bei allen invasiven Maßnahmen sind die DGRh-Empfehlungen zur perioperativen Vorgehensweise bei Patienten, die eine Therapie mit DMARDs und Biologika erhalten, zu beachten [573].

\section{Empfehlung/Statement}

\section{8-28}

Bei Patienten mit axialer SpA und symptomatischer peripherer Arthritis (Statement) oder Enthesitis kann eine lokale Injektion mit Glukokortikoiden erfolgen.

Empfehlungsgrad: 0

Evidenz: 1

\section{Kommentar:}

Die Empfehlung bezüglich der Enthesitis basiert auf einer einzigen kontrollierten Studie, in der eine Glukokortikoidinjektion gegenüber einer Injektion mit einem TNFi verglichen wird. Rando- misierte Studien mit einem Vergleich Glukokortikoidinjektion vs. Plazebo fehlen. Daher wird der Empfehlungsgrad von „ $A$ " auf " 0 “ herabgestuft.

\section{8-29}

Bei Patienten mit axialer SpA und symptomatischer florider Sakroiliitis kann eine Glukokortikoidinjektion in das Sakroiliakalgelenk erfolgen. Empfehlungsgrad: 0

Evidenz: 4

\section{8-30}

Bei Patienten mit AS und einer klinisch symptomatischen Destruktion der Hüftgelenke sollte die Indikation zur Versorgung mit einer Totalendoprothese unabhängig vom Lebensalter gestellt werden.

Empfehlungsgrad: B

Evidenz: 4

\section{Kommentar:}

Die Empfehlung wurde von einem Empfehlungsgrad " $0^{\prime \prime}$ auf eine „B"-Empfehlung hochgestuft, weil es aufgrund ethischer Verpflichtung keine prospektiven Daten geben kann.

\section{8-31}

Die Möglichkeit einer Wirbelsäulenaufrichtungsoperation in einem erfahrenen Zentrum sollte AS-Patienten mit einer erheblichen Wirbelsäulenkyphose und dem Verlust der horizontalen Sicht angeboten werden.

Empfehlungsgrad: Statement

Evidenz: -

\section{3-2\#}

Bei einer raschen Verschlimmerung oder Veränderung der Schmerzsymptomatik der Wirbelsäule sollte neben einer Entzündung auch an eine Fraktur (auch nach geringfügigem Trauma) gedacht werden. Eine entsprechende Diagnostik inklusive Bildgebung (Rö/CT/MRT) sollte zeitnah veranlasst werden. Bei Wirbelsäulenverletzungen (Frakturen) sollte aufgrund des höheren Instabilitätspotenzials nur in Ausnahmefällen eine konservative der operativen Therapie vorgezogen werden. Empfehlungsgrad: $B$

Evidenz: 5
Kommentar zur Empfehlung 3-2:

Die Empfehlung wurde von einem Empfehlungsgrad " 0 " auf eine „B"-Empfehlung hochgestuft, weil es aufgrund ethischer Verpflichtung keine prospektiven Daten geben kann und gleichzeitig Patienten mit einer Wirbelkörperfraktur ein schlechtes Outcome haben.

\# Wiedergabe der Empfehlung aus Abschn. 3

\section{8-32}

Patienten mit axialer SpA und ankylosierter Wirbelsäule und einer Wirbelfraktur sollten in einem spezialisierten operativen Wirbelsäulenzentrum vorgestellt werden.

Empfehlungsgrad: 0

Evidenz: 4

\subsection{Rehabilitation}

\section{Schlüsselfrage 15}

- Welche Effekte haben Trainings- und Rehabilitationsprogramme?

Medizinische Rehabilitationsmaßnahmen wirken sich positiv auf die körperliche Funktionsfähigkeit aus [574]. Ein 3-wöchiges stationäres Rehabilitationsprogramm zeigte gegenüber der üblichen ambulanten Versorgung durch Rheumatologen und Hausärzte noch nach einem Jahr Verbesserungen der Krankheitsaktivität und der Lebensqualität [344]. Es gibt Anhaltspunkte, dass Klima und Umgebung eine Rolle bei der Effektivität von Maßnahmen spielen könnten. So zeigte eine Studie aus Norwegen, dass 16 Wochen nach einer 4-wöchigen stationären Rehabilitationsleistung eine ASAS-20-Ansprechrate von $23 \%$ erwartet werden kann, wenn die Rehabilitationsleistung in Norwegen erbracht wurde, und dass $50 \% \mathrm{zu}$ erwarten sind, wenn die Rehabilitationsleistung in Mittelmeerraum erbracht wurde [575]. Rehabilitationsmaßnahmen wirken besonders gut, wenn sie mit einem verhaltenstherapeutischen Modul während der Rehabilitationsmaßnahme kombiniert sind [576]. Welchen additiven Effekt ein körperliches Training im Vergleich $\mathrm{zu}$ einer Verhaltensschulung hat, ist aber unklar [345]. 
Eine Besonderheit in deutschsprachigen Ländern ist die Heilstollen-Behandlung (teilweise auch als Wannenbad), in deren Rahmen das schwach radioaktive Edelgas Radon über die Haut und Atmung in den Organismus aufgenommen wird. Eine randomisierte Studie untersuchte eine 3-wöchige Rehabilitationsmaßnahme in einem speziellen Kurort (Heilstollen, Radontherapie) im Vergleich zu einem anderen Kurort ohne Radontherapie sowie einer alleinigen ambulanten Physiotherapie, gefolgt von wöchentlichen Gruppen-Physiotherapiesitzungen über 37 Wochen. Die Rehabilitationsmaßnahmen verbesserten Schmerzen und Allgemeinbefinden von AS-Patienten in beiden Gruppen deutlicher und anhaltender als in der Gruppe mit alleiniger Physiotherapie mit einem nichtsignifikanten Vorteil für die nicht verblindet untersuchbare HeilstollenGruppe gegenüber der konventionellen Rehabilitation [332]. In einer damit verbundenen sozioökonomischen Analyse wurde darüber hinaus errechnet, dass bei parallel laufender konventioneller medikamentöser Behandlung die kombinierte Rehabilitation und kontinuierliche Gruppenphysiotherapie ein günstiges Kosten-Nutzen-Verhältnis und einen realen Nutzwert aufweisen [577].

Adäquate Rehabilitationsmaßnahmen sind bei Patienten mit einer hohen Krankheitsaktivität teilweise erst nach Einleitung medikamentöser Maßnahmen durchführbar. Von 60 ASPatienten, die wegen unzureichenden Ansprechens auf NSAR mehrheitlich Bewegungstherapie ablehnten und daraufhin alle Etanercept erhielten, wurde nach Besserung unter 2-monatiger Biologikatherapie bei zufällig ausgewählten 30 Patienten ein 7-tägiges intensives Rehabilitationsprogramm durchgeführt und mit der nur medikamentös behandelten Gruppe verglichen [351]. Nach 6 Monaten zeigte die Rehabilitationsgruppe eine höhere Lebensqualität (EQ-5D) als die Vergleichsgruppe. In einer weiteren randomisierten kontrollierten Studie wurden 62 AS-Patienten mit bestehender und effektiver Therapie mit einem TNFi 3 Studienarmen zugeordnet: (A) intensives Rehabilitationsprogramm (inklusive eines ver- haltenstherapeutischen Moduls), (B) 2wöchentliches Schulungsprogramm und (C) keine zusätzliche Intervention [373]. Nach 6 Monaten war die Reha-Gruppe hinsichtlich Schmerz, BASFI, BASDAI, BASMI, Wirbelsäulen- und Thoraxbeweglichkeit der Kontrollgruppe und bezüglich der meisten Parameter auch der Schulungsgruppe überlegen. Insbesondere Bewegungstherapie, die in Kombination mit anderen adhärenzfördernden Maßnahmen entsprechend einem komplexen rehabilitativen Ansatz durchgeführt wurde, zeigte gegenüber der alleinigen Biologikatherapie zahlreiche zusätzliche Effekte.

Allerdings nimmt nur ein relativ kleiner Teil der Patienten Rehabilitationsmaßnahmen in Anspruch. In einer Befragung unter 400 deutschen Rheumapatienten der Region Halle und einer gleichzeitigen Befragung von internistischen Rheumatologen zeigte sich, dass nur ca. 50\% der AS-Patienten mit Rehabilitationsbedarf auch wirklich eine rehabilitative Leistung beantragen [578].

\section{Empfehlung/Statement}

8-33

Die medizinische Rehabilitation wirkt sich positiv auf die Schmerzen, Beweglichkeit und körperliche Funktionsfähigkeit bei Patienten mit funktionellen Einschränkungen aus. Die Indikation zur Rehabilitation sollte bedarfsorientiert evaluiert werden, auch vor Ablauf des 4-jährigen Regelabstandes zu einer vorausgegangenen medizinischen Rehabilitation.

Empfehlungsgrad: $B$

Evidenz: 2b

\section{Internationale Klassifikation für Funktionsfähigkeit, Behinderung und Gesundheit (ICF)}

\section{Schlüsselfrage 9}

- Welche Probleme der Alltagsfähigkeit und sozialen Teilhabe sollen erfasst werden (ICF)?

\subsection{ICF Core Set für AS}

Patienten mit einer axialen SpA erleben eine vielfältige Beeinträchtigung im Leben durch ihre Erkrankung. In vielen Untersuchungen wird auf die reduzierte Lebensqualität und Funktionsfähigkeit von axSpA-Patienten eingegangen [74, 152, 463, 465]. Studien, die die Gesamtgruppe der axialen SpA untersucht haben, zeigen, dass die Krankheitslast sich bei Patienten mit AS und mit nr-axSpA kaum unterscheidet $[74,75,176]$.

Zur systematischen Erfassung der Beeinträchtigung der Alltagsfähigkeit und sozialen Teilhabe ist die Internationale Klassifikation für Funktionsfähigkeit, Behinderung und Gesundheit (ICF) durch die WHO 2004 entwickelt worden [465]. Die ICF ist in die Komponenten Körperfunktion und Körperstruktur sowie Aktivität und Teilhabe aufgeteilt. Diese Komponenten können von Umweltfaktoren oder personenbezogenen Faktoren beeinflusst werden.

Neben dieser konzeptuellen Anwendung des Modells bietet die gesamte ICF ein Klassifikationssystem mit mehr als 1500 Kategorien, deren Anwendung sich in der Praxis noch nicht allgemein durchgesetzt hat. Für spezifische Erkrankungen existieren sog. „Core Sets“, die die relevanten Beeinträchtigungen für eine bestimmte Erkrankung zusammenfassen. Im Jahr 2009 ist ein ICF Core Set für AS entwickelt worden, das insbesondere für die spezialfachärztliche, therapeutische und rehabilitative Versorgung einen systematischen Zugang zu bedeutenden Aspekten der Funktionsfähigkeit bietet [579]. Die Entwicklung des Core Sets, dessen Themen in einem Delphi-Verfahren zusammengestellt wurden, basiert auf strukturierten Patienteninterviews sowie Expertenmeinungen [580, 581]. In - Tab. 10 sind die Beeinträchtigungen erfasst, die AS-Patienten nach Ansicht von Patientenvertretern und nach Ansicht des Gesundheitspersonals spüren.

In einer anderen Analyse mit einer norwegischen Population wurde auf die Beeinträchtigung nur im Bereich der Aktivität und Teilhabe eingegangen. Die Probleme, die am häufigsten während dieser Interviews genannt wurden, waren: „unterbrochener Schlaf“, „einge- 


\section{Leitlinien}

Tab. 10 Häufigkeit der Funktionseinschränkungen anhand der ICF

ICF-Kategorie

Niederländische

AS-Experten

Norwegische

AS-Patienten [581]

Körperfunktion

\begin{tabular}{l|l|l|}
\hline b130 Psychische Energie und Antrieb & 81
\end{tabular}

b180 Die Selbstwahrnehmung und die Zeitwahrnehmung betreffende Funktionen

\begin{tabular}{l|l|l} 
b280 Schmerz & 97
\end{tabular}

b440 Atmung 58

b4402 Atemtiefe 58

b455 Kardiorespiratorische Belastbarkeit

b640 Sexuelle Funktionen

b660 Fortpflanzungsfunktionen

b710 Gelenkbeweglichkeit

b715 Funktionen der Gelenkstabilität

bb720 Beweglichkeit der Knochen

b730 Muskelkraft

b770 Bewegungsmuster beim Gehen

b780 Gefühl zu Bewegung assoziiert oder Muskel- und Bewegungsschmerzen

b7800 Empfindung von Muskelsteifigkeit

81

Körperstruktur

s220 Augapfel

s410 Kardiovaskuläres System

s420 Immunsystem

s430 Atmungssystem

s710 Kopf- und Halsregion

s720 Schulter

s740 Becken

s750 Untere Extremität

100

83

84

100

$58-100$

$58-$

$-$

s760 Rumpf

s770 Weitere mit der Bewegung in Zusammenhang stehende muskuloskeletale Strukturen

\section{Aktivität und Teilhabe}

d410 Elementare Körperposition wechseln

d4100 Sich hinlegen

d4101 Hocken

d4103 Sitzen

d4105 Sich beugen

d415 In einer Körperposition verbleiben

d4150 In liegender Position verbleiben

d4153 In sitzender Position verbleiben

d4154 In stehender Position verbleiben

d420 Sich verlagern

d4201 Sich beim Liegen verlagern

d430 Gegenstände tragen, bewegen und handhaben

d435 Gegenstände mit den unteren Extremitäten bewegen

d445 Hand- und Armgebrauch

\section{$-$}

d450 Gehen

d455 Sich auf andere Weise fortbewegen 
Tab. 10 (Fortsetzung)

Niederländische

AS-Patienten [581]
AS-Experten

[579]
Norwegische

AS-Patienten [12]

\begin{tabular}{|c|c|c|c|c|c|}
\hline d4551 & \multicolumn{2}{|l|}{ Klettern/steigen } & 78 & - & 39 \\
\hline d4552 & \multicolumn{2}{|l|}{ Rennen } & 93 & - & - \\
\hline d460 & \multicolumn{2}{|l|}{ Sich in verschiedenen Umgebungen fortbewegen } & - & 89 & - \\
\hline d465 & \multicolumn{2}{|l|}{ Sich unter Verwendung von Geräten/Ausrüstung fortbewegen } & - & 86 & - \\
\hline d470 & \multicolumn{2}{|l|}{ Transportmittel benutzen } & - & 84 & - \\
\hline d475 & \multicolumn{2}{|l|}{ Ein Fahrzeug fahren } & 74 & 88 & - \\
\hline d4751 & \multicolumn{2}{|l|}{ Ein motorisiertes Fahrzeug fahren } & 70 & - & - \\
\hline d480 & \multicolumn{2}{|l|}{ Tiere zu Transportzwecken reiten } & - & 81 & - \\
\hline d510 & \multicolumn{2}{|l|}{ Sich waschen } & - & 100 & - \\
\hline d520 & \multicolumn{2}{|l|}{ Seine Körperteile pflegen } & 70 & 96 & - \\
\hline d5204 & \multicolumn{2}{|l|}{ Die Fußnägel pflegen } & 70 & - & - \\
\hline d540 & \multicolumn{2}{|l|}{ Sich kleiden } & 62 & 100 & - \\
\hline d5402 & \multicolumn{2}{|l|}{ Schuhwerk anziehen } & 62 & - & 38 \\
\hline d5403 & \multicolumn{2}{|l|}{ Schuhwerk ausziehen } & 61 & - & - \\
\hline d640 & \multicolumn{2}{|l|}{ Hausarbeiten erledigen } & 86 & 100 & 39 \\
\hline d650 & \multicolumn{2}{|l|}{ Haushaltsgegenstände pflegen } & - & 85 & - \\
\hline d845 & \multicolumn{2}{|l|}{ Eine Arbeit erhalten, behalten und beenden } & - & 94 & - \\
\hline d850 & \multicolumn{2}{|l|}{ Bezahlte Tätigkeit } & 58 & 85 & - \\
\hline d910 & \multicolumn{2}{|l|}{ Gemeinschaftsleben } & 59 & - & 52 \\
\hline d920 & \multicolumn{2}{|l|}{ Erholung und Freizeit } & 82 & 93 & - \\
\hline $\begin{array}{l}\text { Umwelt- } \\
\text { faktoren }\end{array}$ & Förderfaktoren & Barriere & - & - & - \\
\hline e110 & $\begin{array}{l}\text { Produkte und Substanzen für den persönlichen Ver- } \\
\text { brauch }\end{array}$ & - & 76 & 91 & - \\
\hline e115 & $\begin{array}{l}\text { Produkte und Technologien zum persönlichen Gebrauch } \\
\text { im täglichen Leben }\end{array}$ & - & 71 & 86 & - \\
\hline e1150 & Allgemeine Produkte zum persönlichen Gebrauch & - & 64 & - & - \\
\hline e120 & $\begin{array}{l}\text { Produkte und Technologien zur persönlichen Mobilität } \\
\text { drinnen und draußen und zum Transport }\end{array}$ & - & - & 85 & - \\
\hline e135 & Produkte und Technologien für die Erwerbstätigkeit & - & - & 88 & - \\
\hline e225 & - & Klima & 60 & - & - \\
\hline e310 & Engster Familienkreis & - & 89 & 81 & - \\
\hline e320 & Freunde & - & 77 & - & - \\
\hline e325 & $\begin{array}{l}\text { Bekannte, Seinesgleichen (Peers), Kollegen, Nachbarn } \\
\text { und andere Gemeindemitglieder }\end{array}$ & - & 50 & - & - \\
\hline e355 & Fachleute der Gesundheitsberufe & - & 88 & 91 & - \\
\hline e410 & $\begin{array}{l}\text { Individuelle Einstellungen der Mitglieder des engsten } \\
\text { Familienkreises }\end{array}$ & - & 78 & 85 & - \\
\hline e420 & Individuelle Einstellungen von Freunden & - & 62 & - & - \\
\hline e450 & $\begin{array}{l}\text { Individuelle Einstellungen von Fachleuten der Gesund- } \\
\text { heitsberufe }\end{array}$ & - & 73 & 85 & - \\
\hline e460 & Gesellschaftliche Einstellungen & - & - & 92 & - \\
\hline e570 & $\begin{array}{l}\text { Dienste, Systeme und Handlungsgrundsätze der sozia- } \\
\text { len Sicherheit }\end{array}$ & - & - & 100 & - \\
\hline e575 & $\begin{array}{l}\text { Dienste, Systeme und Handlungsgrundsätze der allge- } \\
\text { meinen sozialen Unterstützung }\end{array}$ & - & - & 100 & - \\
\hline e580 & $\begin{array}{l}\text { Dienste, Systeme und Handlungsgrundsätze des Ge- } \\
\text { sundheitswesens }\end{array}$ & - & 55 & 100 & - \\
\hline e590 & $\begin{array}{l}\text { Dienste, Systeme und Handlungsgrundsätze des Ar- } \\
\text { beits- und Beschäftigungswesens }\end{array}$ & - & - & 94 & - \\
\hline
\end{tabular}


schränkte Kopfbewegungen beim Autofahren“, „Einkäufe tragen“ und „Energie für soziale Tätigkeiten" haben [12].

Schon vor 12 Jahren wurde in einer amerikanischen Population gezeigt, wie bedeutsam Beeinträchtigungen im Alltag für AS-Patienten sind [582]. Diese Ergebnisse von 175 AS-Patienten zeigen nicht nur eine Beeinträchtigung der Körperfunktionen (Schmerz, Steifheit), sondern die Patienten waren auch durch $\mathrm{Zu}$ kunftsängste, reduzierte soziale Kontakte und Medikamentennebenwirkungen beeinträchtigt. Diese Erhebung beruhte zwar nicht primär auf der ICF, die hierbei identifizierten Hemmnisse sind jedoch auf die Systematik der ICF übertragbar.

Die Beeinträchtigung ist im Bereich der Körperfunktionen auf die Problembereiche Schmerz und Funktionseinschränkung in der Region Rumpf/ Becken/untere Extremität zurückzuführen. In den Bereichen Aktivität und Teilhabe sind die Limitationen direkt auf Beeinträchtigungen der Körperfunktionen und -strukturen zurückzuführen (Körperposition). Sowohl Freizeit- und Berufsleben als auch die Verrichtung von Hausarbeit werden von der Mehrzahl der Patienten als beeinträchtigt angegeben. Hinsichtlich der Umweltfaktoren werden die Unterstützung und Haltung der Familie, von Freunden und von der Peergroup als wichtig erachtet. Bei den Umweltfaktoren werden fast alle Bereiche überwiegend positiv bewertet. Lediglich das Klima wird als mögliche Barriere empfunden.

Idealerweise sollte die Untersuchung von Patienten sich auf alle Bereiche beziehen, die von den Patienten als problematisch erlebt werden. Die angegebene Beeinträchtigung sollte grundsätzlich aber möglichst mit validen Messinstrumenten überprüft werden. Die im Kapitel Assessments erläuterten Messinstrumente erfassen überwiegend die Bereiche Körperfunktion und Körperstruktur. Die Bereiche Aktivität und Teilhabe werden zwar ebenfalls erfasst, die Aspekte unterscheiden sich zum Teil aber erheblich [583, 584]. Eine Untersuchung mit 522 AS-Patienten aus Kanada und Australien hat gezeigt, dass die Varianz von Messinstrumenten (in der Studie wird dies anhand von BASDAI, BASFI,
ASQoL, EQ-5D gezeigt) erheblich durch Umweltfaktoren und personenbezogene Faktoren (in der ICF als kontextuale Faktoren zusammengefasst) beeinflusst wird [585].

\section{Empfehlung/Statement}

\section{9-1}

Die systematische Erfassung von funktionellen Beeinträchtigungen zur Beurteilung der Krankheitslast bei Patienten mit axialer SpA kann mit der Internationalen Klassifikation für Funktionsfähigkeit, Behinderung und Gesundheit (ICF) erfolgen. Das ICF Core Set für AS gibt auch für relevante Bereiche der Teilhabe eine Orientierung.

Empfehlungsgrad: Statement Evidenz: -

\subsection{Aktivitäten und Teilhabe}

\section{Schlüsselfrage 2}

- Welche Interventionen können die Aktivität und Teilhabe (Partizipation) positiv beeinflussen?

Die Beeinträchtigungen von Patienten mit AS im Alltag können durch systematische Anwendung der ICF erhoben werden (s. Abschn. 9.2). In diesem Kapitel wird auf die Beeinträchtigung im Berufsleben und auf mögliche Interventionen eingegangen. Für AS-Patienten haben verschiedene soziale Rollen eine höhere subjektive Bedeutung als für Vergleichspersonen aus der Bevölkerung, aber ihre Zufriedenheit mit ihrem Ausfüllen dieser Rollen ist deutlich geringer als bei den Kontrollpersonen [586]. Die standardisierte Beschäftigungsrate von AS-Patienten in Deutschland bei Patienten im Alter von 20 bis 59 Jahren zwischen 1993 und 2001 lag bei 0,94 [13]. Bei Patienten mit einer Krankheitsdauer $>10$ Jahre lag das relative Risiko, berufstätig zu sein, von Patienten mit AS im Vergleich zu RA bei 1,42. Vergleichende Daten der deutschen rheumatologischen Kerndokumentation ambulant betreuter AS-Patienten im erwerbsfähigen Alter der Jahre 2000 und 2012 zeigen, dass sich die Anteile der Erwerbstätigen unter der Frauen von 51 auf $56 \%$ und deutlicher bei den Män- nern von 54 auf $71 \%$ erhöhten [314, 587]. Entsprechend verminderte sich in diesem Zeitraum der Anteil der männlichen AS-Patienten mit Erwerbminderungsrente von 20,8 auf $12,4 \%$, während er sich unter den Frauen nicht änderte $(16,7 \%$ gegenüber $16,5 \%)$. Weibliches Geschlecht, niedriger Bildungsstand und höheres Lebensalter sind mit einem höheren Risiko der vorzeitigen Berentung assoziiert [20]. Daten aus den USA, Holland und der Türkei zeichnen ein ähnliches Bild der Beschäftigungssituation [11, 14, 588-592]. Es waren 13,2\% der Patienten mit AS in einer US-amerikanischen Kohorte dauerhaft erwerbsunfähig, und insgesamt $24,3 \%$ hatten jemals eine Zahlung wegen Arbeitsunfähigkeit erhalten [588]. Risikofaktoren sind höheres Lebensalter, geringere Bildung, Anzahl der Komorbiditäten und körperlich anstrengende Berufe. Das Risiko, innerhalb von 4 Jahren nach Diagnose die Arbeitszeit reduzieren zu müssen, war assoziiert mit einer größeren Einschränkung der körperlichen Funktionsfähigkeit und einem höheren Schmerzlevel. Im Vergleich zur US-amerikanischen Normalbevölkerung war dies signifikant erhöht [11]. Dies lag besonders an der Altersgruppe $\geq 45$ Jahre mit $\geq 20$ Jahren Krankheitsdauer. In einer türkischen Kohorte gaben $44 \%$ der Patienten an, die Arbeit gewechselt zu haben und zum Zeitpunkt der Befragung einen „leichteren“ Job zu verrichten [589].

Es gibt Untersuchungen für TNFi, die zeigen, dass eine suffiziente antientzündliche Therapie zu besserer Arbeitsfähigkeit und Produktivität sowie zu einem geringeren Risiko, den Job zu verlieren, führt [480, 481, 483].

Positive Effekte auf die Arbeitsfähigkeit und Produktivität wurden auch für ein strukturiertes Patientenschulungsprogramm für Patienten mit AS im Rahmen einer stationären Rehabilitation nachgewiesen: Noch 1 Jahr danach wurden bei Schulungsteilnehmern gegenüber Rehabilitanden ohne Schulung weniger Arbeitsunfähigkeit, seltener Rentenanträge und geringere indirekte Krankheitskosten durch Arbeitsausfälle festgestellt [593, 594]. Die multimodale Rehabilitation, die neben einer suffizienten antientzündlichen Therapie ein 
intensives Bewegungsprogramm und eine strukturierte Patientenschulung für Patienten mit AS einschließt, verbessert somit die Aktivität und Teilhabe am Erwerbsleben.

Zunehmend relevant sind auch stärker berufsbezogene Therapieelemente der Rehabilitation wie arbeitsplatzbezogenes Training einschließlich ergonomischer Beratung und Sozialberatung, die individuell nach den empfundenen Arbeitsbelastungen, den funktionellen Einschränkungen und vor allem den beruflichen Kontextfaktoren der Rehabilitanden angeboten werden [595].

\section{Empfehlung/Statement}

\section{9-2}

Patienten sollen darüber informiert werden, dass Aktivität und Teilhabe durch pharmakologische und nichtpharmakologische Maßnahmen positiv beeinflusst werden. Zu den nichtpharmakologischen Maßnahmen gehören:

- multimodale Rehabilitation mit intensiver Bewegungstherapie (B),

- strukturierte Patientenschulung (B),

- berufsbezogene Therapieelemente in der Rehabilitation (Expertenkonsens).

\section{Empfehlungsgrad: $B$}

Evidenz: 1/2+

\section{Patienteninformation}

\subsection{Strukturiertes Schulungs- programm}

\section{Schlüsselfrage 18}

- Welche Effekte haben strukturierte Patientenschulungsprogramme?

Von der DGRh ist ein strukturiertes Patientenschulungsprogramm für Patienten mit AS entwickelt worden. Das Programm wird sowohl ambulant als auch stationär angeboten. Eine kontrollierte Studie verglich AS-Patienten mit und ohne Schulungsprogramm im Rahmen einer stationären Rehabilitation [593, 594]. Schulungsteilnehmer hatten noch nach einem Jahr eine bessere Krankheitsbewältigung durch erhöhte Selbstwirksamkeit sowie geringere Krankheitskosten vor allem durch weniger Arbeitsausfälle. Die Durchführung eines strukturierten Schulungsprogramms führte bei spanischen AS-Patienten dazu, dass die Patienten mehr Informationen $\mathrm{zu}$ ihrer Erkrankung hatten und dass Bewegungsübungen häufiger durchgeführt wurden (selbstberichtet) [596]. Allerdings waren die Effekte auf die Krankheitsaktivität und körperliche Funktionsfähigkeit nur marginal.

\section{Empfehlung/Statement}

10-1

Patienten mit einer axialen SpA sollten an einem strukturierten Schulungsprogramm teilnehmen, da die Krankheitsbewältigung verbessert wird und die Krankheitskosten reduziert werden. Empfehlungsgrad: $B$

Evidenz: 1/2+

\section{Kommentar:}

Die zugrunde liegende Studie hat ein kontrolliertes, quasi-randomisiertes, prospektives multizentrisches Design.

\subsection{Gesundheitsfördernde Verhaltensweise}

\section{Schlüsselfrage 17}

- Welche gesundheitsfördernde Verhaltensweise soll empfohlen werden (Nichtrauchen, Ernährung, [Freizeit-] Sport, Selbsthilfegruppe)?

Rauchende AS-Patienten haben mehr Schmerzen, eine höhere Krankheitsaktivität und einen größeren röntgenologischen Schaden als nicht rauchende Patienten, und die Höhe des Zigarettenkonsums korreliert mit der Höhe der Krankheitsaktivität [597, 598]. Rauchende Patienten mit axialer SpA haben eine größere Einschränkung der körperlichen Funktionsfähigkeit als nicht rauchende Patienten (BASFI 5,5 vs. 3,8; $p=0,002)[70,319]$. Das Ausmaß struktureller Läsionen (gemessen am BASRI) ist bei rauchenden Patienten höher als bei nicht rauchenden Patienten (OR 4,72) [599]. Diese Ergebnisse konnten in den 2 großen europäischen Frühkohorten bestätigt werden [224, 600, 601].
Zum Einfluss von Ernährung gibt es keine Daten.

Daten über den Einfluss von (Freizeit-) Sport liegen ebenfalls nicht vor. Es gibt kontrollierte Studien, die Patienten ohne und mit regelmäßiger Bewegung sowie Patienten mit regelmäßiger Bewegung im Vergleich zu strukturierten Therapieprogrammen untersuchen (s. Abschn. 8.3).

\section{Empfehlung/Statement}

\section{0-2}

Patienten mit axSpA sollen darüber informiert werden, dass neben den allgemeinen gesundheitlichen Risiken des Rauchens speziell für sie stärkere Einbußen der Funktionsfähigkeit, eine stärkere röntgenologische Progression und ein schlechteres Therapieansprechen auf Biologika im Vergleich zu Nichtrauchern bestehen.

Empfehlungsgrad: B

Evidenz: $2 b$

\subsection{Selbsthilfegruppe}

Die Datenlage zur Effektivität von Selbsthilfegruppen ist spärlich, da keine kontrollierten Studien oder größere Kohortenstudien vorliegen. Es liegen keine Daten zum Einfluss von Selbsthilfegruppen auf den Krankheitsverlauf vor. Experten gehen allerdings davon aus, dass Selbsthilfegruppen hilfreich in der Unterstützung von Patienten sein können [602]. Das betrifft sowohl die gegenseitige Beratung als auch die gemeinsame Organisation von Bewegungstherapie.

\section{Empfehlung/Statement}

\section{0-3}

Patientenorganisationen und Selbsthilfegruppen können das Management der Erkrankung unterstützen. Empfehlungsgrad: Statement Evidenz: - 


\section{Korrespondenzadresse}

PD Dr. med. U. Kiltz

Rheumazentrum Ruhrgebiet, Ruhr-Universität Bochum

Claudiusstr. 45, 44649 Herne, Deutschland uta.kiltz@elisabethgruppe.de

\section{Einhaltung ethischer Richtlinien}

Interessenkonflikt. Angaben zum Interessenkonflikt aller Leitlinienautoren sind im Leitlinienreport unter https://www.awmf.org/leitlinien/detail/ll/060-003. html einsehbar.

Für diesen Beitrag wurden von den Autoren keine Studien an Menschen oder Tieren durchgeführt. Für die aufgeführten Studien gelten die jeweils dort angegebenen ethischen Richtlinien.

\section{Literatur}

1. Braun J, Sieper J (2007) Ankylosing spondylitis. Lancet 369(9570):1379-1390

2. Rudwaleit $M$ et al (2009) The development of Assessment of SpondyloArthritis international Society classification criteria for axial spondyloarthritis (part II): validation and final selection. Ann Rheum Dis 68(6):777-783

3. Rudwaleit M et al (2009) The development of Assessment of SpondyloArthritis international Society classification criteria for axial spondyloarthritis (part I): classification of paper patients by expert opinion including uncertainty appraisal. Ann Rheum Dis 68(6):770-776

4. Dean LE et al (2014) Global prevalence of ankylosing spondylitis. Rheumatol (oxford) 53(4):650-657

5. Braun J et al (1998) Prevalence of spondylarthropathies in HLA-B27 positive and negative blood donors. Arthritis Rheum 41(1):58-67

6. Almodovar R et al (2011) Phenotypic differences between familial versus sporadic ankylosing spondylitis: a cross-sectional Spanish registry of spondyloarthropathies (REGISPONSER). Clin Exp Rheumatol 29(5):822-827

7. Chen HA et al (2012) Clinical, functional, and radiographic differences among juvenile-onset, adult-onset, and late-onset ankylosing spondylitis. JRheumatol 39(5):1013-1018

8. Jamalyaria F et al (2017) Ethnicity and disease severity in ankylosing spondylitis a cross-sectional analysis of three ethnic groups. Clin Rheumatol 36(10):2359-2364

9. Skare TL et al (2012) Effect of age at disease onset in the clinical profile of spondyloarthritis: a study of 1424 Brazilian patients. Clin Exp Rheumatol 30(3):351-357

10. Landewe Ret al (2009) Physical function in ankylosing spondylitis is independently determined by both disease activity and radiographic damage of the spine. Ann Rheum Dis 68(6):863-867

11. Ward MM et al (2008) Impact of ankylosing spondylitis on work and family life: comparisons with the US population. Arthritis Rheum 59(4):497-503

12. Dagfinrud $\mathrm{H}$ et al (2005) Impact of functional impairment in ankylosing spondylitis:impairment, activity limitation, and participation restrictions. JRheumatol 32(3):516-523

13. Mau W et al (2005) Employment across chronic inflammatory rheumatic diseases and comparison with the general population. J Rheumatol 32(4):721-728

14. Boonen A et al (2010) Impact of ankylosing spondylitis on sick leave, presenteeism and unpaid productivity, and estimation of the societal cost. Ann Rheum Dis 69(6):1123-1128

15. Calin A et al (1980) The prevalence and nature of back pain in an industrial complex: a questionnaire and radiographic and $\mathrm{HLA}$ analysis. Spine (phila Pa 1976) 5(2):201-205

16. Feldtkeller E et al (2003) Age at disease onset and diagnosis delay in HLA-B27 negative vs. positive patients with ankylosing spondylitis. Rheumatol Int 23(2):61-66

17. van der Linden SM et al (1984) The risk of developing ankylosing spondylitis in HLA-B27 positive individuals. A comparison of relatives of spondylitis patients with the general population Arthritis Rheum 27(3):241-249

18. Sorensen J, Hetland ML, D. all departments of rheumatology (2015) in, Diagnostic delay in patients with rheumatoid arthritis, psoriatic arthritis and ankylosing spondylitis: results from the Danish nationwide DANBIO registry. Ann Rheum Dis 74(3):e12

19. Burgos-Varga $R$ et al (2016) The prevalence and clinical characteristics of nonradiographic axial spondyloarthritis among patients with inflammatory back pain in rheumatology practices: a multinational, multicenter study. Arthritis Res Ther 18(1):132

20. Zink A et al (2000) Disability and handicap in rheumatoid arthritis and ankylosing spondylitis-results from the German rheumatological database. German Collaborative Arthritis Centers. JRheumatol 27(3):613-622

21. van der Heijde D et al (2017) 2016 update of the ASAS-EULAR management recommendations for axial spondyloarthritis. Ann Rheum Dis 76(6):978-991

22. Rojas-Vargas M et al (2009) First signs and symptoms of spondyloarthritis - data from an inception cohort with a disease course of two years or less (REGISPONSER-Early). Rheumatol (oxford) 48(4):404-409

23. Hamilton L et al (2014) The prevalence of inflammatory back pain in a UK primary care population. Rheumatol (oxford) 53(1):161-164

24. Calin A et al (1977) Clinical history as a screening test for ankylosing spondylitis. JAMA 237(24):2613-2614

25. Rudwaleit M et al (2006) Inflammatory back pain in ankylosing spondylitis: a reassessment of the clinical history for application as classification and diagnostic criteria. Arthritis Rheum 54(2):569-578

26. Sieper J et al (2009) New criteria for inflammatory back pain in patients with chronic back pain: a rea patient exercise by experts from the Assessment of SpondyloArthritis international Society (ASAS). Ann Rheum Dis 68(6):784-788

27. Solmaz D et al (2014) Performance of different criteria sets for inflammatory back pain in patients with axial spondyloarthritis with and without radiographic sacroiliitis. Clin Rheumatol 33(10):1475-1479

28. Gazeau P et al (2018) Classification criteria versus physician's opinion for considering a patient with inflammatory back pain as suffering from spondyloarthritis. Joint Bone Spine 85(1):85-91
29. Machado Petal (2010) Both structural damage and inflammation of the spine contribute to impairment of spinal mobilityin patients with ankylosing spondylitis. Ann Rheum Dis 69(8):1465-1470

30. Weber U et al (2012) Anterior chest wall inflammation by whole-body magnetic resonance imaging in patients with spondyloarthritis: lack of association between clinical and imaging findings in a cross-sectional study. Arthritis Res Ther 14(1):R3

31. Payet J et al (2012) Prevalence and clinical characteristics of dactylitis in spondylarthritis: a descriptive analysis of 275 patients. Clin Exp Rheumatol 30(2):191-196

32. Navarro-Compan V et al (2015) Sponyloarthritis features forecasting the presence of HLA-B27 or sacroiliitis on magnetic resonance imaging in patients with suspected axial spondyloarthritis: results from a cross-sectional study in the ESPeranza Cohort. Arthritis Res Ther 17:265

33. Bethi S et al (2013) Functional limitations due to axial and peripheral joint impairments in patients with ankylosing spondylitis: are focused measures more informative? Arthritis Care Res (hoboken) 65(4):607-614

34. Aydin SZ et al (2016) A relationship between spinal new bone formation in ankylosing spondylitis and the sonographically determined Achilles tendon enthesophytes. Rheumatol Int 36(3):397-404

35. Ruta S et al (2011) Prevalence of subclinical enthesopathy in patients with spondyloarthropathy: an ultrasound study. J Clin Rheumatol 17(1):18-22

36. Maksymowych WP et al (2009) Development and validation of the Spondyloarthritis Research Consortium of Canada (SPARCC) Enthesitis Index. Ann Rheum Dis 68(6):948-953

37. Heuft-Dorenbosch L et al (2003) Assessment of enthesitis in ankylosing spondylitis. Ann Rheum Dis 62(2):127-132

38. Tournadre A et al (2013) Differences between women and men with recent onset axial spondyloarthritis: results from the DESIR cohort. Arthritis Care Res (hoboken) 65(9):1482-1489

39. Webers $C$ et al (2016) Gender-attributable differences in outcome of ankylosing spondylitis: long-term results from the Outcome in Ankylosing Spondylitis International Study. Rheumatol (oxford) 55(3):419-428

40. van der Horst-Bruinsma IE et al (2013) Female patients with ankylosing spondylitis: analysis of the impact of gender across treatment studies. Ann Rheum Dis 72(7):1221-1224

41. Landi $M$ et al (2016) Gender differences among patients with primary ankylosing spondylitis and spondylitis associated with psoriasis and inflammatory bowel disease in an iberoamerican spondyloarthritis cohort. Medicine (Baltimore) 95(51):e5652

42. Sidiropoulos PI et al (2008) Evidence-based recommendations for the management of ankylosing spondylitis: systematic literature search of the 3 Initiative in Rheumatology involving a broad panel of experts and practising rheumatologists. Rheumatol (oxford) 47(3):355-361

43. Rudwaleit $M$ et al (2004) How to diagnose axial spondyloarthritis early. Ann Rheum Dis 63(5):535-543

44. Karberg K et al (2005) Bone loss is detected more frequently in patients with ankylosing spondylitis with syndesmophytes. J Rheumatol 32(7):1290-1298

45. Wang D et al (2017) Bone edema on magnetic resonance imaging is highly associated with low bone mineral density in patients with ankylosing spondylitis. PLoS ONE 12(12):e189569 
46. Briot $\mathrm{K}$ et al (2016) Bone loss in patients with early inflammatory back pain suggestive of spondyloarthritis: results from the prospective DESIR cohort. Rheumatol (oxford) 55(2):335-342

47. Gratacos J et al (1999) Significant loss of bone mass in patients with early, active ankylosing spondylitis: a followup study. Arthritis Rheum 42(11):2319-2324

48. Prieto-Alhambra D et al (2015) Ankylosing spondylitis confers substantially increased risk of clinical spine fractures: a nationwide case-control study. Osteoporos Int 26(1):85-91

49. Jun JB et al (2006) Femoral bone mineral density is associated with vertebral fractures in patients with ankylosing spondylitis: a cross-sectional study. JRheumatol 33(8):1637-1641

50. Vosse D et al (2009) Ankylosing spondylitis and the risk of fracture: results from a large primary carebased nested case-control study. Ann Rheum Dis 68(12):1839-1842

51. Deminger A et al (2017) Which measuring site in ankylosing spondylitis is best to detect bone loss and what predicts the decline: results from a 5 -year prospective study. Arthritis Res Ther 19(1):273

52. Montala $\mathrm{N}$ et al (2011) Prevalence of vertebral fractures by semiautomated morphometry in patients with ankylosing spondylitis. J Rheumatol 38(5):893-897

53. Westerveld LA, Verlaan JJ, Oner FC (2009) Spinal fractures in patients with ankylosing spinal disorders: a systematic review of the literature on treatment, neurological status and complications. Eur Spine J 18(2):145-156

54. Dursun $\mathrm{N}$ et al (2015) Risk of falls in patients with ankylosing spondylitis. J Clin Rheumatol 21(2):76-80

55. Protopopov M et al (2017) Relevance of structural damage in the sacroiliac joints for the functional status and spinal mobility in patients with axial spondyloarthritis: results from the German Spondyloarthritis Inception Cohort. Arthritis Res Ther 19(1):240

56. Haywood KL et al (2004) Spinal mobility in ankylosing spondylitis: reliability, validity and responsiveness. Rheumatol (oxford) 43(6):750-757

57. Wanders A et al (2005) Association between radiographic damage of the spine and spinal mobility for individual patients with ankylosing spondylitis: can assessment of spinal mobility be a proxy for radiographic evaluation? Ann Rheum Dis 64(7):988-994

58. Heuft-Dorenbosch L et al (2004) Measurement of spinal mobility in ankylosing spondylitis: comparison of occiput-to-wall and tragus-to-wall distance. J Rheumatol 31(9):1779-1784

59. Deyo RA, Diehr P, Patrick DL (1991) Reproducibility and responsiveness of health status measures. Statistics and strategies for evaluation. Control Clin Trials 12(4Suppl):142S-158S

60. Pile KD et al (1991) Clinical assessment of ankylosing spondylitis: a study of observer variation in spinal measurements. Br J Rheumatol 30(1):29-34

61. Gladman DD et al (2007) International spondyloarthritis interobserver reliability exercise-the INSPIRE study: I. Assessment of spinal measures. JRheumatol 34(8):1733-1739

62. KennedyLGetal (1995) Ankylosing spondylitis: the correlation between a new metrology score and radiology. Br JRheumatol 34(8):767-770

63. Chandran V et al (2007) Relationship between spinal mobility and radiographic damage in ankylosing spondylitis and psoriatic spondylitis: a comparative analysis. J Rheumatol 34(12):2463-2465
64. Shin JK et al (2014) Correlation between clinical outcome and spinopelvic parameters in ankylosing spondylitis. Eur Spine J 23(1):242-247

65. Jenkinson TRetal(1994)Defining spinalmobilityin ankylosing spondylitis (AS). The Bath AS Metrology Index. JRheumatol 21(9):1694-1698

66. Calin A et al (1994) A new approach to defining functional ability in ankylosing spondylitis: the development of the Bath Ankylosing Spondylitis Functional Index.J Rheumatol 21(12):2281-2285

67. Garrett $S$ et al (1994) A new approach to defining disease status in ankylosing spondylitis: the Bath Ankylosing Spondylitis Disease Activity Index. JRheumatol 21(12):2286-2291

68. Ruof J, Sangha O, Stucki G (1999) Evaluation of a German version of the Bath Ankylosing Spondylitis Functional Index (BASFI) and Dougados Functional Index (D-FI). Z Rheumatol 58(4):218-225

69. Boonen A et al (2009) Spinal radiographic changes in ankylosing spondylitis:association with clinical characteristics and functional outcome. JRheumatol 36(6):1249-1255

70. Ward MMetal (2005) Riskfactors for functional limitations in patients with long-standing ankylosing spondylitis. Arthritis Rheum 53(5):710-717

71. Daltroy LH et al (1990) A modification of the Health Assessment Questionnaire for the spondyloarthropathies. JRheumatol 17(7):946-950

72. Vander Cruyssen Betal (2007) The epidemiology of ankylosing spondylitis and the commencement of anti-TNF therapy in daily rheumatology practice. Ann Rheum Dis 66(8):1072-1077

73. Brophy S, Calin A (2001) Ankylosing spondylitis: interaction between genes, joints, age at onset, and disease expression. J Rheumatol 28(10):2283-2288

74. Rudwaleit M et al (2009) The early disease stage in axial spondylarthritis: results from the German Spondyloarthritis Inception Cohort. Arthritis Rheum 60(3):717-727

75. Kiltz $U$ et al (2012) Do patients with nonradiographic axial spondylarthritis differ from patients with ankylosing spondylitis? Arthritis Care Res (hoboken) 64(9):1415-1422

76. Stolwijk C et al (2015) Prevalence of extra-articular manifestations in patients with ankylosing spondylitis: a systematic review and meta-analysis. Ann Rheum Dis 74(1):65-73

77. Collantes E et al (2007) Disease pattern of spondyloarthropathies in Spain: description of the first national registry (REGISPONSER) extended report. Rheumatol (oxford) 46(8):1309-1315

78. BremanderAetal (2011) Population-based estimates of common comorbidities and cardiovascular disease in ankylosing spondylitis. Arthritis Care Res (hoboken) 63(4):550-556

79. Chorus AM et al (2003) Quality of life and work in patients with rheumatoid arthritis and ankylosing spondylitis of working age. Ann Rheum Dis 62(12):1178-1184

80. Sampaio-Barros PD et al (2013) An analysis of 372 patients with anterior uveitis in a large Ibero-American cohort of spondyloarthritis: the RESPONDIA Group. Clin Exp Rheumatol 31(4):484-489

81. Canoui-Poitrine F et al (2012) Prevalence and factors associated with uveitis in spondylarthritis patients in France: results from an observational survey. Arthritis Care Res (hoboken) 64(6):919-924

82. Zeboulon N, Dougados M, Gossec L (2008) Prevalence and characteristics of uveitis in the spondyloarthropathies: a systematic literature review. Ann Rheum Dis 67(7):955-959
83. Essers I et al (2015) Characteristics associated with the presence and development of extraarticular manifestations in ankylosing spondylitis: 12-year results from OASIS. Rheumatol (oxford) 54(4):633-640

84. Robinson PC et al (2015) Genetic dissection of acute anterior uveitis reveals similarities and differences in associations observed with ankylosing spondylitis. Arthritis Rheumatol 67(1):140-151

85. Rosenbaum JT (1992) Acute anterior uveitis and spondyloarthropathies. Rheum Dis Clin North Am 18(1):143-151

86. Accorinti $M$ et al (2010) Clinical features and prognosis of HLA B27-associated acute anterior uveitis in an Italian patient population. Ocul Immunol Inflamm 18(2):91-96

87. Munoz-Fernandez S et al (2003) Sulfasalazine reduces the number of flares of acute anterior uveitis over a one-year period. J Rheumatol 30(6):1277-1279

88. Benitez-Del-Castillo JM et al (2000) Sulfasalazine in the prevention of anterior uveitis associated with ankylosing spondylitis. Eye (Lond) $14(\mathrm{Pt}$ 3A):340-343

89. Nguyen QD et al (2016) Adalimumab for prevention of uveitic flare in patients with inactive noninfectious uveitis controlled by corticosteroids (VISUAL II): a multicentre, double-masked, randomised, placebo-controlled phase 3 trial. Lancet 388(10050):1183-1192

90. Braun J et al (2005) Decreased incidence of anterior uveitis in patients with ankylosing spondylitis treated with the anti-tumor necrosis factor agents infliximab and etanercept. Arthritis Rheum 52(8):2447-2451

91. Sieper J et al (2010) Analysis of uveitis rates across all etanercept ankylosing spondylitis clinical trials. Ann Rheum Dis 69(1):226-229

92. Gao X et al (2012) Clinical and economic burden of extra-articular manifestations in ankylosing spondylitis patients treated with anti-tumor necrosis factor agents. J Med Econ 15(6):1054-1063

93. Lie E et al (2017) Tumour necrosis factor inhibitor treatment and occurrence of anterior uveitis in ankylosing spondylitis: results from the Swedish biologics register. Ann Rheum Dis 76(9):1515-1521

94. Wendling D et al (2014) Comparing the risk of developing uveitis in patients initiating anti-tumor necrosis factor therapy for ankylosing spondylitis: an analysis of a large US claims database. Curr Med Res Opin 30(12):2515-2521

95. Rudwaleit M et al (2009) Adalimumab effectively reduces the rate of anterior uveitis flares in patients with active ankylosing spondylitis: results of a prospective open-label study. Ann Rheum Dis 68(5):696-701

96. Dick AD et al (2013) Secukinumab in the treatment of noninfectious uveitis: results of three randomized, controlled clinical trials. Ophthalmology 120(4):777-787

97. Letko $E$ et al (2015) Efficacy and safety of intravenous secukinumab in noninfectious uveitis requiring steroid-sparing immunosuppressive therapy. Ophthalmology 122(5):939-948

98. Mielants Het al (1996) Course of gut inflammation in spondylarthropathies and therapeutic consequences. Baillieres Clin Rheumatol 10(1):147-164

99. Mielants $\mathrm{H}$ et al (1995) The evolution of spondyloarthropathies in relation to gut histology. III. Relation between gut and joint. J Rheumatol 22(12):2279-2284 
100. D'Inca R et al (2009) Articular manifestations in inflammatory bowel disease patients: a prospective study. Dig Liver Dis 41(8):565-569

101. Peeters $\mathrm{H}$ et al (2008) Clinical and genetic factors associated with sacroiliitis in Crohn's disease. JGastroenterol Hepatol 23(1):132-137

102. Vavricka SR et al (2011) Frequency and risk factors for extraintestinal manifestations in the Swiss inflammatory bowel disease cohort. Am J Gastroenterol 106(1):110-119

103. El Miedany Y et al (2006) The gastrointestinal safety and effect on disease activity of etoricoxib, a selective cox- 2 inhibitor in inflammatory bowel diseases. Am J Gastroenterol 101(2):311-317

104. Hoffmann JC et al (2008) Clinical practice guideline on diagnosis and treatment of Crohn's disease. ZGastroenterol 46(9):1094-1146

105. Braun J et al (2007) Differences in the incidence of flares or new onset of inflammatory bowel diseases in patients with ankylosing spondylitis exposed to therapy with anti-tumor necrosis factor alpha agents. Arthritis Rheum 57(4):639-647

106. Toussirot E et al (2012) Development of inflammatory bowel disease during anti-TNF-alpha therapy for inflammatory rheumatic disease. A nationwide series. Joint Bone Spine 79(5):457-463

107. Hueber $W$ et al (2012) Secukinumab, a human anti-IL-17A monoclonal antibody, for moderate to severe Crohn's disease: unexpected results of a randomised, double-blind placebo-controlled trial.Gut 61(12):1693-1700

108. Bergfeldt $L$ et al (1982) Ankylosing spondylitis: an important cause of severe disturbances of the cardiac conduction system. Prevalence among 223 pacemaker-treated men. Am J Med 73(2):187-191

109. O'Neill TW, Bresnihan B (1992) The heart in ankylosing spondylitis. Ann Rheum Dis 51(6):705-706

110. Bengtsson K et al (2018) Risk of cardiac rhythm disturbances and aortic regurgitation in different spondyloarthritis subtypes in comparison with general population: a register-based study from Sweden. Ann Rheum Dis 77(4):541-548

111. Sampaio-Barros PD et al (2007) Pulmonary involvement in ankylosing spondylitis. Clin Rheumatol 26(2):225-230

112. El Maghraoui A et al (2004) Lung findings on thoracichigh-resolution computed tomographyin patients with ankylosing spondylitis. Correlations with disease duration, clinical findings and pulmonary function testing. Clin Rheumatol 23(2):123-128

113. Souza ASJr.etal (2004)Pulmonaryabnormalities in ankylosing spondylitis: inspiratory and expiratory high-resolution CT findings in 17 patients. J Thorac Imaging 19(4):259-263

114. Gratacos J et al (1997) Secondary amyloidosis in ankylosing spondylitis. A systematic survey of 137 patients using abdominal fat aspiration. JRheumatol 24(5):912-915

115. LeeSHetal(2013) Renal involvement in ankylosing spondylitis: prevalence, pathology, response to TNF-a blocker. Rheumatol Int 33(7):1689-1692

116. Jakobsen AK et al (2014) Is nephrolithiasis an unrecognized extra-articular manifestation in ankylosing spondylitis? A prospective populationbased Swedish national cohort study with matched general population comparator subjects. PLoSONE 9(11):e113602

117. Molto A et al (2016) Prevalence of comorbidities and evaluation of their screening in spondyloarthritis: results of the international crosssectional ASAS-COMOSPA study. Ann Rheum Dis 75(6):1016-1023
118. Hmamouchiletal (2016) Vitamin D, diseaseactivity and comorbidities in early spondyloarthritis. Clin Exp Rheumatol 34(3):396-403

119. Meesters JJ et al (2014) The risk for depression in patients with ankylosing spondylitis:a populationbased cohort study. Arthritis Res Ther 16(5):418

120. Li Y et al (2012) Sleep disturbances are associated with increased pain, disease activity, depression, and anxiety in ankylosing spondylitis: a casecontrol study. Arthritis Res Ther 14(5):R215

121. Dhakad U et al (2015) Sexual dysfunctions and lower urinary tract symptoms in ankylosing spondylitis. Int J Rheum Dis 18(8):866-872

122. Dau JD et al (2018) Opioid analgesic use in patients with Ankylosing Spondylitis: an analysis of the prospective study of outcomes in an Ankylosing Spondylitis cohort. JRheumatol 45(2):188-194

123. Maas F et al (2016) Obesity is common in axial Spondyloarthritis and is associated with poor clinical outcome. J Rheumatol43(2):383-387

124. Baraliakos X et al (2018) Patients with fibromyalgia rarely fulfil classification criteria for axial spondyloarthritis. Rheumatol (oxford) 57(9):1541-1547

125. Molto A et al (2018) Evaluation of the impact of concomitant fibromyalgia on TNF alpha blockers effectiveness in axial spondyloarthritis: results of a prospective, multicentre study. Ann Rheum Dis 77(4):533-540

126. Macfarlane GJ et al (2017) Co-occurrence and characteristics of patients with axial Spondyloarthritis who meet criteria for Fibromyalgia: results from a UK national register. Arthritis Rheumatol 69(11):2144-2150

127. Peters MJ et al (2004) Cardiovascular risk profile of patients with spondylarthropathies, particularly ankylosing spondylitis and psoriatic arthritis. Semin Arthritis Rheum 34(3):585-592

128. Haroon NN et al (2015) Patients with Ankylosing Spondylitis have increased cardiovascular and cerebrovascular mortality: a population-based study. Ann Intern Med 163(6):409-416

129. Heeneman S, Daemen MJ (2007) Cardiovascular risks in spondyloarthritides. Curr Opin Rheumatol 19(4):358-362

130. Arida A et al (2015) Subclinical atherosclerosis is not accelerated in patients with Ankylosing Spondylitis with low disease activity: new data and metaanalysis of published studies. J Rheumatol 42(11):2098-2105

131. Chen $Y$ et al (2015) Left ventricular myocardial dysfunction and premature atherosclerosis in patients with axial spondyloarthritis. Rheumatol (oxford) 54(2):292-301

132. Choe JY et al (2008) No differences of carotid intima-media thickness between young patients with ankylosing spondylitis and healthy controls. Joint Bone Spine 75(5):548-553

133. Gonzalez-Juanatey C et al (2009) The high prevalence of subclinical atherosclerosis in patients with ankylosing spondylitis without clinically evident cardiovascular disease. Medicine (Baltimore) 88(6):358-365

134. Peters MJ et al (2010) Signs of accelerated preclinical atherosclerosis in patients with ankylosing spondylitis. JRheumatol 37(1):161-166

135. Bengtsson Ketal(2017) Areankylosing spondylitis, psoriatic arthritis and undifferentiated spondyloarthritis associated with an increased risk of cardiovascular events? A prospective nationwide population-based cohort study. Arthritis Res Ther 19(1):102

136. Peters MJ et al (2010) Ankylosing spondylitis: a risk factor for myocardial infarction? Ann Rheum Dis 69(3):579-581
137. Mathieu S et al (2011) Cardiovascular profile in ankylosing spondylitis: a systematic review and meta-analysis. Arthritis Care Res (hoboken) 63(4):557-563

138. van Halm VP et al (2006) Increased disease activity is associated with a deteriorated lipid profile in patients with ankylosing spondylitis. Ann Rheum Dis 65(11):1473-1477

139. Berg IJ et al (2015) Disease activity in ankylosing spondylitis and associations to markers of vascular pathology and traditional cardiovascular disease risk factors: a cross-sectional study. J Rheumatol 42(4):645-653

140. Spanakis E et al (2006) Modest but sustained increase of serum high density lipoprotein cholesterol levels in patients with inflammatory arthritides treated with infliximab. J Rheumatol 33(12):2440-2446

141. van Eijk IC et al (2009) Improvement of lipid profile is accompanied by atheroprotective alterations in high-density lipoprotein composition upon tumor necrosis factor blockade: a prospective cohort study in ankylosing spondylitis. Arthritis Rheum 60(5):1324-1330

142. van Eijk IC et al (2009) Microvascular function is impaired in ankylosing spondylitis and improves after tumour necrosis factor alpha blockade. Ann Rheum Dis 68(3):362-366

143. Peters MJ et al (2010) EULAR evidence-based recommendations for cardiovascular risk management in patients with rheumatoid arthritis and other forms of inflammatory arthritis. Ann Rheum Dis 69(2):325-331

144. Bianchi WA et al (2014) Assessment of fatigue in a large series of 1492 Brazilian patients with Spondyloarthritis. Mod Rheumatol 24(6):980-984

145. Alkan BM et al (2013) Fatigue and correlation with disease-specific variables, spinal mobility measures, and health-related quality of life in ankylosing spondylitis. Mod Rheumatol 23(6):1101-1107

146. van Tubergen A et al (2002) Assessment of fatigue in patients with ankylosing spondylitis: a psychometric analysis. Arthritis Rheum 47(1):8-16

147. Jones SD et al (1996) Fatigue in ankylosing spondylitis: its prevalence and relationship to disease activity, sleep, and other factors. JRheumatol 23(3):487-490

148. Bedaiwi $\mathrm{M}$ et al (2015) Fatigue in Ankylosing Spondylitis and Nonradiographic axial Spondyloarthritis:analysisfromalongitudinal observation cohort.JRheumatol 42(12):2354-2360

149. Dagfinrud $\mathrm{H}$ et al (2005) Fatigue in patients with ankylosing spondylitis: a comparison with the general population and associations with clinical and self-reported measures. Arthritis Rheum 53(1):5-11

150. Chauffier Ketal (2013) Fatigue in spondyloarthritis: a marker of disease activity. A cross-sectional study of 266 patients. Clin Exp Rheumatol 31(6):864-870

151. Gossec L et al (2016) Fatigue in early axial spondyloarthritis. Results from the French DESIR cohort. Joint Bone Spine 83(4):427-431

152. van der Heijde DM et al (2009) Physical function, disease activity, and health-related quality-of-life outcomes after 3 years of adalimumab treatment in patients with ankylosing spondylitis. Arthritis Res Ther 11(4):R124

153. Revicki DA et al (2008) Adalimumab reduces pain, fatigue, and stiffness in patients with ankylosing spondylitis: results from the adalimumab trial evaluating long-term safety and efficacy for ankylosing spondylitis (ATLAS). J Rheumatol 35(7):1346-1353 
154. Espahbodi S et al (2017) Fatigue contributes to work productivity impairment in patients with axial spondyloarthritis: a cross-sectional UK study. Clin Exp Rheumatol 35(4):571-578

155. Zochling J, Braun J (2009) Mortality in rheumatoid arthritis and ankylosing spondylitis. Clin Exp Rheumatol 27(4Suppl 55):S127-30

156. Brown WM, Doll R (1965) Mortality from cancer and other causes after radiotherapy for ankylosing spondylitis. Br Med J 2(5474):1327-1332

157. Exarchou $S$ et al (2016) Mortality in ankylosing spondylitis: results from a nationwide populationbased study. Ann Rheum Dis 75(8):1466-1472

158. Oza A et al (2017) Survival benefit of statin use in ankylosing spondylitis: a general population-based cohort study. Ann Rheum Dis 76(10):1737-1742

159. Bakland G, Gran JT, Nossent JC (2011) Increased mortality in ankylosing spondylitis is related to disease activity. Ann Rheum Dis 70(11):1921-1925

160. ZinkA, Minden K (2010) Entzündlich rheumatische Erkrankungen. Gesundheitsberichterstattung des Bundes, Bd. 49. R.-K.I., Berlin

161. Wysham KD et al (2017) Cervical spinal fracture and other diagnoses associated with mortality in hospitalized Ankylosing Spondylitis patients. Arthritis Care Res (hoboken) 69(2):271-277

162. Wick RR et al (2008) Increased risk of myeloid leukaemia in patients with ankylosing spondylitis following treatment with radium-224. Rheumatol (oxford) 47(6):855-859

163. Rudwaleit $M$ et al (2011) The Assessment of SpondyloArthritis International Society classification criteria for peripheral spondyloarthritis and for spondyloarthritis in general. Ann Rheum Dis 70(1):25-31

164. Amor B, Dougados M, Mijiyawa M (1990) Criteria of the classification of spondylarthropathies. Rev Rhum Mal Osteoartic 57(2):85-89

165. Dougados Metal (1991) The European Spondylarthropathy Study Group preliminary criteria for the classification of spondylarthropathy. Arthritis Rheum 34(10):1218-1227

166. Chenot JF et al (2017) Non-specific low back pain. Dtsch Arztebl Int 114(51-52):883-890

167. Aydin SZ et al (2012) Validation of the ASAS criteria and definition of a positive MRI of the sacroiliac joint in an inception cohort of axial spondyloarthritis followed up for 8 years. Ann Rheum Dis 71(1):56-60

168. Sepriano A et al (2016) Predictive validity of the ASAS classification criteria for axial and peripheral spondyloarthritis after follow-up in the ASAS cohort: a final analysis. Ann Rheum Dis 75(6):1034-1042

169. Molto A et al (2013) Performances of the Assessment of SpondyloArthritis International Society axial spondyloarthritis criteria for diagnostic and classification purposes in patients visiting a rheumatologist because of chronic back pain: results from a multicenter, cross-sectional study. Arthritis Care Res (hoboken) 65(9):1472-1481

170. Molto A et al (2014) Evaluation of the validity of the different arms of the ASAS set of criteria for axial spondyloarthritis and description of the different imaging abnormalities suggestive of spondyloarthritis: data from the DESIR cohort. Ann Rheum Dis 74(4):746-751

171. Rudwaleit Metal (2005) The challenge of diagnosis and classification in early ankylosing spondylitis: do we need new criteria? Arthritis Rheum 52(4):1000-1008. https://doi.org/10.1002/art. 20990
172. Ez-Zaitouni Z et al (2017) Presence of multiple spondyloarthritis (SpA) features is important but not sufficient for a diagnosis of axial spondyloarthritis: data from the SPondyloArthritis Caught Early (SPACE) cohort. Ann Rheum Dis 76(6):1086-1092

173. Bakker PA et al (2016) Are additional tests needed to rule out axial Spondyloarthritis in patients ages 16-45 years with short-duration chronic back pain and maximally one Spondyloarthritis feature? Arthritis Care Res (hoboken) 68(11):1726-1730

174. Joven BE et al (2017) Diagnostic value and validity of early Spondyloarthritis features: results from a national Spanish cohort. Arthritis Care Res (hoboken) 69(6):938-942

175. van der Linden S, Valkenburg HA, Cats A (1984) Evaluation of diagnostic criteria for ankylosing spondylitis. A proposal for modification of the New York criteria. Arthritis Rheum 27(4):361-368

176. Wallman JK et al (2015) Comparison of non-radiographic axial spondyloarthritis and ankylosing spondylitis patients-baseline characteristics, treatment adherence, and development of clinical variables during three years of anti-TNF therapy in clinical practice. Arthritis Res Ther 17(1):378

177. Malaviya AN et al (2015) Comparison of patients with ankylosing spondylitis (AS) and nonradiographic axial spondyloarthritis ( $\mathrm{nr}-\mathrm{axSpA}$ ) from a single rheumatology clinic in New Delhi. Int JRheum Dis 18(7):736-741

178. Glintborg B et al (2017) Ankylosing Spondylitis versus Nonradiographic Axial Spondyloarthritis: Comparison of Tumor Necrosis Factor Inhibitor Effectiveness and Effect of HLA-B27 Status. An Observational Cohort Study from the Nationwide DANBIO Registry. JRheumatol 44(1):59-69

179. Sieper J, Rudwaleit M (2005) Early referral recommendations for ankylosing spondylitis (including pre-radiographic and radiographic forms) in primary care. Ann Rheum Dis 64(5):659-663

180. Heuft-Dorenbosch L et al (2007) Performance of various criteria sets in patients with inflammatory back pain of short duration; the Maastricht early spondyloarthritis clinic. Ann Rheum Dis 66(1):92-98

181. Dean LE, Macfarlane GJ, Jones GT (2016) Differences in the prevalence of ankylosing spondylitis in primary and secondary care: only one-third of patients are managed in rheumatology. Rheumatol (oxford) 55(10):1820-1825

182. Deodhar A et al (2016) Ankylosing spondylitis diagnosis in US patients with back pain: identifying providers involved and factors associated with rheumatology referral delay. Clin Rheumatol 35(7):1769-1776

183. Underwood MR, Dawes P (1995) Inflammatory back pain in primary care. $\mathrm{Br} J$ Rheumatol 34(11):1074-1077

184. StrandVetal (2013) Prevalence of axial spondyloarthritis in United States rheumatology practices: Assessment of SpondyloArthritis International Society criteria versus rheumatology expert clinical diagnosis. Arthritis Care Res (hoboken) 65(8):1299-1306

185. Liao ZT et al (2009) An epidemiological survey of low back pain and axial spondyloarthritis in a Chinese Han population. Scand J Rheumatol 38(6):455-459

186. Weisman MH, Witter JP, Reveille JD (2013) The prevalence of inflammatory backpain: populationbased estimates from the US National Health and Nutrition Examination Survey, 2009-10. Ann Rheum Dis 72(3):369-373
187. Jois RN, Macgregor AJ, Gaffney K (2008) Recognition of inflammatory back pain and ankylosing spondylitis in primary care. Rheumatol (oxford) 47(9):1364-1366

188. Brandt HC et al (2007) Performance of referral recommendations in patients with chronic back pain and suspected axial spondyloarthritis. Ann Rheum Dis 66(11):1479-1484

189. Hermann J et al (2009) Early spondyloarthritis: usefulness of clinical screening. Rheumato (oxford) 48(7):812-816

190. Braun A et al (2011) Identifying patients with axial spondyloarthritis in primary care: how useful are items indicative of inflammatory back pain? Ann Rheum Dis 70(10):1782-1787

191. Poddubnyy Det al (2011) Evaluation of 2 screening strategies for early identification of patients with axial spondyloarthritis in primary care. JRheumatol 38(11):2452-2460

192. Sampaio-Barros PD et al (2010) Undifferentiated spondyloarthritis: a longterm followup. JRheumatol 37(6):1195-1199

193. Freeston J et al (2007) Ankylosing spondylitis, HLAB27 positivity and the need for biologic therapies. Joint Bone Spine 74(2):140-143

194. Chung HY et al (2011) HLA-B27 positive patients differ from HLA-B27 negative patients in clinical presentation and imaging: results from the DESIR cohort of patients with recent onset axial spondyloarthritis. Ann Rheum Dis 70(11):1930-1936

195. van Onna M et al (2011) HLA-B27 and gender independently determine the likelihood of a positive MRI of the sacroiliac joints in patients with early inflammatory back pain: a 2-year MRI follow-upstudy. Ann Rheum Dis 70(11):1981-1985

196. Linssen A (1990) B27+disease versus B27-disease. Scand J Rheumatol Suppl 87:111-118 (discussion 118-9)

197. Khan MA, Kushner I, Braun WE (1977) Comparison of clinical features in HLA-B27 positive and negative patients with ankylosing spondylitis. Arthritis Rheum 20(4):909-912

198. Kim TJ et al (2009) HLA-B27 homozygosity has no influence on clinical manifestations and functional disability in ankylosing spondylitis. Clin Exp Rheumatol 27(4):574-579

199. Lin H, Gong YZ (2017) Association of HLA-B27 with ankylosing spondylitis and clinical features of the HLA-B27-associated ankylosing spondylitis: a meta-analysis. Rheumatol Int 37(8):1267-1280

200. Spoorenberg A et al (1999) Relative value of erythrocyte sedimentation rate and C-reactive protein in assessment of disease activity in ankylosing spondylitis. JRheumatol 26(4):980-984

201. Braun A et al (2013) Optimizing the identification of patients with axial spondyloarthritis in primary care-the case for a two-step strategy combining the most relevant clinical items with HLA B27. Rheumatol (oxford) 52(8):1418-1424

202. van Hoeven $L$ et al (2014) Identifying axial spondyloarthritis in Dutch primary care patients, ages 20-45 years, with chronic low back pain. Arthritis Care Res (hoboken) 66(3):446-453

203. Sieper J et al (2013) Comparison of two referral strategies for diagnosis of axial spondyloarthritis: the Recognising and Diagnosing Ankylosing Spondylitis Reliably (RADAR) study. Ann Rheum Dis 72(10):1621-1627

204. Abawi $O$ et al (2017) Evaluation of multiple referral strategies for axial spondyloarthritis in the SPondyloArthritis Caught Early (SPACE) cohort. RMD Open 3(1):e389

205. Baraliakos X et al (2008) The relationship between inflammation and new bone formation in patients 
with ankylosing spondylitis. Arthritis Res Ther 10(5):R104

206. Maksymowych WP et al (2009) Inflammatory lesions of the spine on magnetic resonance imaging predict the development of new syndesmophytes in ankylosing spondylitis: evidence of a relationship between inflammation and new bone formation. Arthritis Rheum 60(1):93-102

207. Chiowchanwisawakit $P$ et al (2011) Focal fat lesions at vertebral corners on magnetic resonance imaging predict the development of new syndesmophytes in ankylosing spondylitis. Arthritis Rheum 63(8):2215-2225

208. Pedersen SJ et al (2011) Radiographic progression is associated with resolution of systemic inflammation in patients with axial spondylarthritis treated with tumornecrosis factor alpha inhibitors: a study of radiographic progression, inflammation on magnetic resonance imaging, and circulating biomarkers of inflammation, angiogenesis, and cartilage and bone turnover. Arthritis Rheum 63(12):3789-3800

209. Pedersen SJetal (2011) Resolution ofinflammation following treatment of ankylosing spondylitis is associated with new bone formation. J Rheumatol 38(7):1349-1354

210. Baraliakos X et al (2007) Progression of radiographic damage in patients with ankylosing spondylitis: defining the central role of syndesmophytes. Ann Rheum Dis 66(7):910-915

211. Diarra Detal(2007)Dickkopf-1 is a master regulator of joint remodeling. Nat Med 13(2):156-163

212. Daoussis D et al (2010) Evidence that Dkk-1 is dysfunctional in ankylosing spondylitis. Arthritis Rheum 62(1):150-158

213. Appel H et al (2009) Altered skeletal expression of sclerostin and its link to radiographic progression in ankylosing spondylitis. Arthritis Rheum 60(11):3257-3262

214. Mau W et al (1988) Clinical features and prognosis of patients with possible ankylosing spondylitis. Results of a 10-year followup. J Rheumatol 15(7):1109-1114

215. Poddubnyy D et al (2011) Rates and predictors of radiographic sacroiliitis progression over 2 years in patients with axial spondyloarthritis. Ann Rheum Dis 70(8):1369-1374

216. Dougados M et al (2017) Sacroiliac radiographic progression in recent onset axial spondyloarthritis: the 5-year data of the DESIR cohort. Ann Rheum Dis 76(11):1823-1828

217. Wang R, Gabriel SE, Ward MM (2016) Progression of Nonradiographic axial Spondyloarthritis to Ankylosing Spondylitis:a population-based cohort study. Arthritis Rheumatol 68(6):1415-1421

218. Baraliakos X et al (2009) Development of a radiographic scoring tool for ankylosing spondylitis only based on bone formation: addition of the thoracic spine improves sensitivity to change. Arthritis Rheum 61(6):764-771

219. Lee JS et al (2015) Relationship between cervical sagittal alignment and quality of life in ankylosing spondylitis. Eur Spine J24(6):1199-1203

220. Baraliakos X et al (2011) The natural course of radiographic progression in ankylosing spondylitis: differences between genders and appearance of characteristic radiographic features. Curr Rheumatol Rep 13(5):383-387

221. Poddubnyy D et al (2016) High disease activity according to the Ankylosing Spondylitis Disease Activity Score is associated with accelerated radiographic spinal progression in patients with early axial spondyloarthritis: results from the
GErman SPondyloarthritis Inception Cohort. Ann Rheum Dis 75(12):2114-2118

222. van Tubergen A et al (2012) Development of new syndesmophytes and bridges in ankylosing spondylitis and their predictors: a longitudinal study. Ann Rheum Dis 71(4):518-523

223. RamiroSetal (2014) Higher disease activityleads to more structural damage in the spine in ankylosing spondylitis: 12-year longitudinal data from the OASIS cohort. Ann Rheum Dis 73(8):1455-1461

224. Poddubnyy D et al (2012) Baseline radiographic damage, elevated acute-phase reactant levels, and cigarette smoking status predict spinal radiographic progression in early axial spondylarthritis. Arthritis Rheum 64(5):1388-1398

225. van der Heijde D et al (2008) Radiographic findings following two years of infliximab therapy in patients with ankylosing spondylitis. Arthritis Rheum 58(10):3063-3070

226. MacKay K et al (1998) The Bath Ankylosing Spondylitis Radiology Index (BASRI): a new, validated approach to disease assessment. Arthritis Rheum 41(12):2263-2270

227. Averns HL et al (1996) Radiological outcome in ankylosing spondylitis: use of the Stoke Ankylosing Spondylitis Spine Score (SASSS). Br J Rheumatol 35(4):373-376

228. Creemers MCetal (2005) Assessment of outcome in ankylosing spondylitis: an extended radiographic scoring system. Ann Rheum Dis 64(1):127-129

229. Salaffi F et al (2007) Radiological scoring methods for ankylosing spondylitis: a comparison between the Bath Ankylosing Spondylitis Radiology Index and the modified Stoke Ankylosing Spondylitis Spine Score. Clin Exp Rheumatol 25(1):67-74

230. de Bruin F et al (2016) Prevalence of degenerative changes of the spine on magnetic resonance images and radiographs in patients aged 16-45 years with chronic back pain of short duration in the Spondyloarthritis Caught Early (SPACE) cohort Rheumatol (oxford) 55(1):56-65

231. Vosse D et al (2007) Comparing morphometric X-ray absorptiometry and radiography in defining vertebral wedge fractures in patients with ankylosing spondylitis. Rheumatol (oxford) 46(11):1667-1671

232. Diekhoff T et al (2017) Comparison of MRI with radiography for detecting structural lesions of the sacroiliac joint using CT as standard of reference: results from the SIMACT study. Ann Rheum Dis 76(9):1502-1508

233. Baraliakos X et al (2005) Assessment of acute spinal inflammation in patients with ankylosing spondylitis by magnetic resonance imaging: a comparison between contrast enhanced T1 and short tau inversion recovery (STIR) sequences. Ann Rheum Dis 64(8):1141-1144

234. Rudwaleit Met al (2009) Defining active sacroiliitis on magnetic resonance imaging (MRI) for classification of axial spondyloarthritis: a consensual approach by the ASAS/OMERACT MRI group. Ann Rheum Dis 68(10):1520-1527

235. Weber U et al (2009) Sensitivity and specificity of spinal inflammatory lesions assessed by whole-body magnetic resonance imaging in patients with ankylosing spondylitis or recentonset inflammatory back pain. Arthritis Rheum 61(7):900-908

236. Althoff CE et al (2013) Active inflammation and structural change in early active axial spondyloarthritis as detected by whole-body MRI. Ann Rheum Dis 72(6):967-973

237. Weber $U$ et al (2009) Validation of whole-body againstconventionalmagneticresonanceimaging for scoring acute inflammatory lesions in the sacroiliac joints of patients with spondylarthritis. Arthritis Rheum 61(7):893-899

238. Weber $U$ et al (2010) Assessment of active spinal inflammatory changes in patients with axial spondyloarthritis: validation of whole body MRI against conventional MRI. Ann Rheum Dis 69(4):648-653

239. Bollow M et al (2000) Quantitative analyses of sacroiliac biopsies in spondyloarthropathies: $T$ cells and macrophages predominate in early and active sacroiliitis - cellularity correlates with the degree of enhancement detected by magnetic resonance imaging. Ann Rheum Dis 59(2):135-140

240. Gong Y et al (2012) Ten years' experience with needle biopsy in the early diagnosis of sacroiliitis. Arthritis Rheum 64(5):1399-1406

241. Muche B et al (2003) Anatomic structures involved in early- and late-stage sacroiliitis in spondylarthritis: a detailed analysis by contrastenhanced magnetic resonance imaging. Arthritis Rheum 48(5):1374-1384

242. Madsen KB, Jurik AG (2010) Magnetic resonance imaging grading system for active and chronic spondylarthritis changes in the sacroiliac joint. Arthritis Care Res (hoboken) 62(1):11-18

243. Madsen KB, Egund N, Jurik AG (2010) Grading of inflammatory disease activity in the sacroiliac joints with magnetic resonance imaging: comparison between short-tau inversion recovery and gadolinium contrast-enhanced sequences. JRheumatol 37(2):393-400

244. Marzo-Ortega H et al (2009) Baseline and 1-year magnetic resonance imaging of the sacroiliac joint and lumbar spine in very early inflammatory back pain. Relationship between symptoms, HLA-B27 and disease extent and persistence. Ann Rheum Dis 68(11):1721-1727

245. Song IH et al (2011) Relationship between active inflammatory lesions in the spine and sacroiliac joints and new development of chronic lesions on whole-body MRI in early axial spondyloarthritis: results of the ESTHER trial at week 48. Ann Rheum Dis 70(7):1257-1263

246. Hu Z et al (2016) Backfill is a specific sign of axial spondyloarthritis seen on MRI. Joint Bone Spine 83(2):179-183

247. Wick MC et al (2010) Erosions are the most relevant magnetic resonance imaging features in quantification of sacroiliac joints in ankylosing spondylitis. JRheumatol 37(3):622-627

248. WeberUetal (2013) Developmentand validation of a magnetic resonance imaging reference criterion for defining a positive sacroiliac joint magnetic resonance imaging finding in spondyloarthritis. Arthritis Care Res (hoboken) 65(6):977-985

249. Weber $U$ et al (2010) The diagnostic utility of magnetic resonance imaging in spondylarthritis: an international multicenter evaluation of one hundred eighty-seven subjects. Arthritis Rheum 62(10):3048-3058

250. Weber $U$ et al (2010) Assessment of structural lesions in sacroiliac joints enhances diagnostic utility of magnetic resonance imaging in early spondylarthritis. Arthritis Care Res (hoboken) 62(12):1763-1771

251. Madsen KB, Schiottz-Christensen B, Jurik AG (2010) Prognostic significance of magnetic resonance imaging changes of the sacroiliac joints in spondyloarthritis - a followup study. J Rheumatol 37(8):1718-1727

252. Bennett AN et al (2008) Severity of baseline magnetic resonance imaging-evident sacroiliitis and HLA-B27 status in early inflammatory back 
pain predict radiographically evident ankylosing spondylitis at eight years. Arthritis Rheum 58(11):3413-3418

253. Pedersen SJ et al (2016) Course of magnetic resonance imaging-detected inflammation and structural lesions in the sacroiliac joints of patients in the randomized, double-blind, placebo-controlled Danish Multicenter study of Adalimumab in Spondyloarthritis, as assessed by the berlin and Spondyloarthritis research consortium of Canada methods. Arthritis Rheumatol 68(2):418-429

254. BaraliakosXetal (2005)Inflammationinankylosing spondylitis: a systematic description of the extent and frequency of acute spinal changes using magnetic resonance imaging. Ann Rheum Dis 64(5):730-734

255. Rennie WJ et al (2009) Magnetic resonance imaging assessment of spinal inflammation in ankylosing spondylitis: standard clinical protocols may omit inflammatory lesions in thoracic vertebrae. Arthritis Rheum 61(9):1187-1193

256. Appel H et al (2006) Correlation of histopathological findings and magnetic resonance imaging in the spine of patients with ankylosing spondylitis. Arthritis Res Ther 8(5):R143

257. Jevtic Vet al (2000) Marginal erosive discovertebral "Romanus" lesions in ankylosing spondylitis demonstrated by contrast enhanced Gd-DTPA magnetic resonance imaging. Skeletal Radiol 29(1):27-33

258. Romanus R, Yden S(1952) Destructiveand ossifying spondylitic changes in rheumatoid ankylosing spondylitis (pelvo-spondylitis ossificans). Acta Orthop Scand 22(2):88-99

259. Bennett AN et al (2009) Evaluation of the diagnostic utility of spinal magnetic resonance imaging in axial spondylarthritis. Arthritis Rheum 60(5):1331-1341

260. Kenny JB, Hughes PL, Whitehouse GH (1990) Discovertebral destruction in ankylosing spondylitis: the role of computed tomography and magnetic resonance imaging. Br J Radiol 63(750):448-455

261. Kabasakal Y, Garrett SL, Calin A (1996) The epidemiology of spondylodiscitis in ankylosing spondylitis-a controlled study. Br J Rheumatol 35(7):660-663

262. Bennett AN et al (2010) The fatty Romanus lesion: a non-inflammatory spinal MRI lesion specific for axial spondyloarthropathy. Ann Rheum Dis 69(5):891-894

263. Braun J et al (2004) Analysing chronic spinal changes in ankylosing spondylitis: a systematic comparison of conventional $x$ rays with magnetic resonance imaging using established and new scoring systems. Ann Rheum Dis 63(9):1046-1055

264. Braun J et al (2003) Magnetic resonance imaging examinations of the spine in patients with ankylosing spondylitis, before and after successful therapy with infliximab: evaluation of a new scoring system. Arthritis Rheum 48(4):1126-1136

265. van der Heijde $D$ et al (2007) Is there a preferred method for scoring activity of the spine by magnetic resonance imaging in ankylosing spondylitis? J Rheumatol 34(4):871-873

266. Maksymowych WP et al (2005) Spondyloarthritis research Consortium of Canada magnetic resonance imaging index for assessment of sacroiliac joint inflammation in ankylosing spondylitis. Arthritis Rheum 53(5):703-709

267. Maksymowych WP et al (2007) Validation of the spondyloarthritis research consortium of Canada magnetic resonance imaging spinal inflammation index: is it necessary to score the entire spine? Arthritis Rheum 57(3):501-507
268. Lukas C et al (2007) Scoring inflammatory activity of the spine by magnetic resonance imaging in ankylosing spondylitis: a multireader experiment. JRheumatol 34(4):862-870

269. Treitl M et al (2008) Magnetic resonance imaging assessment of spinal inflammation in patients treated for ankylosing spondylitis. J Rheumatol 35(1):126-136

270. Kiltz $U$ et al (2012) The degree of spinal inflammation is similar in patients with axial spondyloarthritis who report high or low levels of disease activity: a cohort study. Ann Rheum Dis 71(7):1207-1211

271. Madsen KB, Jurik AG (2010) MRI grading method for active and chronic spinal changes in spondyloarthritis. Clin Radiol 65(1):6-14

272. Weber U et al (2015) Does spinal MRI add incremental diagnostic value to MRI of the sacroiliac joints alone in patients with nonradiographic axial spondyloarthritis? Ann Rheum Dis 74(6):985-992

273. Hermann KG et al (2012) Descriptions of spinal MRI lesions and definition of a positive MRI of the spine in axial spondyloarthritis: a consensual approach by the ASAS/OMERACT MRI study group. Ann Rheum Dis 71(8):1278-1288

274. Rudwaleit M et al (2008) MRl in predicting a major clinical response to anti-tumour necrosis factor treatment in ankylosing spondylitis. Ann Rheum Dis 67(9):1276-1281

275. Braun J et al (2006) Major reduction in spinal inflammation in patients with ankylosing spondylitis after treatment with infliximab: results of a multicenter, randomized, double-blind, placebocontrolled magnetic resonance imaging study. Arthritis Rheum 54(5):1646-1652

276. Baraliakos X et al (2005) Outcome of patients with active ankylosing spondylitis after two years of therapy with etanercept: clinical and magnetic resonance imaging data. Arthritis Rheum 53(6):856-863

277. Baraliakos X et al (2005) Magnetic resonance imaging examinations of the spine in patients with ankylosing spondylitis before and after therapy with the tumor necrosis factor alpha receptor fusion protein etanercept. Arthritis Rheum 52(4):1216-1223

278. Sieper J et al (2005) Persistent reduction of spinal inflammation as assessed by magnetic resonance imaging in patients with ankylosing spondylitis after 2 yrs of treatment with the anti-tumour necrosis factor agent infliximab. Rheumatol (oxford) 44(12):1525-1530

279. Jarrett SJ et al (2009) MRI and clinical findings in patients with ankylosing spondylitis eligible for anti-tumour necrosis factor therapy after a short course of etoricoxib. Ann Rheum Dis 68(9):1466-1469

280. Feydy A et al (2012) Comparative study of MRI and power Doppler ultrasonography of the heel in patients with spondyloarthritis with and without heel pain and in controls. Ann Rheum Dis 71(4):498-503

281. Spadaro A et al (2009) Sonographic-detected joint effusion compared with physical examination in the assessment of sacroiliac joints in spondyloarthritis. Ann Rheum Dis 68(10):1559-1563

282. Unlu E, Pamuk ON, Cakir N (2007) Color and duplex Doppler sonography to detect sacroiliitis and spinal inflammation in ankylosing spondylitis. Can this method reveal response to anti-tumor necrosis factor therapy? J Rheumatol 34(1):110-116

283. Aydin SZ et al (2010) Validation of ultrasound imaging for Achilles entheseal fibrocartilage in bovines and description of changes in humans with spondyloarthritis. Ann Rheum Dis 69(12):2165-2168

284. D'Agostino MA et al (2011) How to diagnose spondyloarthritis early? Accuracy of peripheral enthesitis detection by power Doppler ultrasonography. Ann Rheum Dis 70(8):1433-1440

285. de Miguel E et al (2011) Diagnostic accuracy of enthesis ultrasound in the diagnosis of early spondyloarthritis. Ann Rheum Dis 70(3):434-439

286. de Miguel E et al (2009) Validity of enthesis ultrasound assessment in spondyloarthropathy. Ann Rheum Dis 68(2):169-174

287. Ruyssen-Witrand A et al (2017) Relationships between ultrasound enthesitis, disease activity and axial radiographic structural changes in patients with early spondyloarthritis: data from DESIR cohort. RMD Open 3(2):e482

288. Song $\mathrm{IH}$ et al (2008) The diagnostic value of scintigraphy in assessing sacroiliitis in ankylosing spondylitis: a systematic literature research. Ann Rheum Dis 67(11):1535-1540

289. Song IHetal (2010) Limited diagnostic value of unilateral sacroiliitis in scintigraphy in assessing axial spondyloarthritis.J Rheumatol 37(6):1200-1202

290. Devauchelle-Pensec V et al (2012) Computed tomography scanning facilitates the diagnosis of sacroiliitis in patients with suspected spondylarthritis: results of a prospective multicenter French cohort study. Arthritis Rheum 64(5):1412-1419

291. Strobel K et al (2010) 18F-fluoride PET/CT for detection of sacroiliitis in ankylosing spondylitis. Eur J Nucl Med Mol Imaging 37(9):1760-1765

292. Dougados Met al (1999) Clinical relevance of C-reactive protein in axial involvement of ankylosing spondylitis. JRheumatol 26(4):971-974

293. BenhamouM,GossecL,Dougados M(2010)Clinical relevance of $\mathrm{C}$-reactive protein in ankylosing spondylitis and evaluation of the NSAIDs/coxibs' treatment effect on C-reactive protein. Rheumatol (oxford) 49(3):536-541

294. Kroon F et al (2012) Continuous NSAID use reverts the effects of inflammation on radiographic progression in patients with ankylosing spondylitis. Ann Rheum Dis 71(10):1623-1629

295. Poddubnyy D et al (2012) Effect of non-steroidal anti-inflammatory drugs on radiographic spinal progression in patients with axial spondyloarthritis: results from the German Spondyloarthritis Inception Cohort. Ann Rheum Dis 71(10):1616-1622

296. Poddubnyy DA et al (2010) Comparison of a high sensitivity and standard $C$ reactive protein measurement in patients with ankylosing spondylitis and non-radiographic axial spondyloarthritis. Ann Rheum Dis 69(7):1338-1341

297. de Vries MKet al (2009) Erythrocyte sedimentation rate, C-reactive protein level, and serum amyloid a protein for patient selection and monitoring of anti-tumor necrosis factor treatment in ankylosing spondylitis. Arthritis Rheum 61(11):1484-1490

298. Klingberg Eetal (2017) A longitudinal study of fecal calprotectin and the development of inflammatory bowel disease in ankylosing spondylitis. Arthritis Res Ther 19(1):21

299. Cypers H et al (2016) Elevated calprotectin levels reveal bowel inflammation in spondyloarthritis. Ann Rheum Dis 75(7):1357-1362

300. van der Heijde D et al (2009) ASDAS, a highly discriminatory ASAS-endorsed disease activity score in patients with ankylosing spondylitis. Ann Rheum Dis 68(12):1811-1818

301. Brandt J et al (2003) Adaption and validation of the Bath Ankylosing Spondylitis Disease Activity 
Index (BASDAI) for use in Germany. Z Rheumatol 62(3):264-273

302. Pavy S, Brophy S, Calin A (2005) Establishment of the minimum clinically important difference for the bath ankylosing spondylitis indices: a prospective study. JRheumatol 32(1):80-85

303. Brandt J et al (2000) Successful treatment of active ankylosing spondylitis with the antitumor necrosis factor alpha monoclonal antibody infliximab. Arthritis Rheum 43(6):1346-1352

304. Berthelot JM et al (2008) High intraindividual week-to-week variability in BASDAI and BASFI values: are several evaluations needed before starting or stopping TNFalpha antagonist therapy for spondyloarthropathies? Joint Bone Spine 75(2):167-171

305. Song IH et al (2009) Comparison of the Bath Ankylosing Spondylitis Disease Activity Index and a modified version of the index in assessing disease activity in patients with ankylosing spondylitis without peripheral manifestations. Ann Rheum Dis 68(11):1701-1707

306. Machado P et al (2015) Calculating the ankylosing spondylitis disease activity score if the conventional c-reactive protein level is below the limit of detection or if high-sensitivity c-reactive protein is used: an analysis in the DESIR cohort. Arthritis Rheumatol 67(2):408-413

307. Machado P et al (2011) Ankylosing Spondylitis Disease Activity Score (ASDAS): defining cut-off values for disease activity states and improvement scores. Ann Rheum Dis 70(1):47-53

308. Nas K et al (2010) Discrimination ability of ASDAS estimating disease activity status in patients with ankylosing spondylitis. Int J Rheum Dis 13(3):240-245

309. Pedersen SJ et al (2010) Responsiveness of the Ankylosing Spondylitis Disease Activity Score (ASDAS) and clinical and MRI measures of disease activity in a 1-year follow-up study of patients with axial spondyloarthritis treated with tumour necrosis factor alpha inhibitors. Ann Rheum Dis 69(6):1065-1071

310. Aydin SZ et al (2010) Active disease requiring TNF-alpha-antagonist therapy can be well discriminated with different ASDAS sets: a prospective, follow-up of disease activity assessment in ankylosing spondylitis. Clin Exp Rheumatol 28(5):752-755

311. Vastesaeger $\mathrm{N}$ et al (2014) ASDAS high disease activity versus BASDAI elevation in patients with ankylosing spondylitis as selection criterion for anti-TNF therapy. Reumatol Clin 10(4):204-209

312. Kilic E et al (2015) Discriminant validity of the Ankylosing Spondylitis Disease Activity Score (ASDAS) in patients with non-radiographic axial spondyloarthritis and ankylosing spondylitis: a cohort study. Rheumatol Int 35(6):981-989

313. Kilic G, Kilic E, Ozgocmen S (2017) Is there any gender-specific difference in the cut-off values of ankylosing spondylitis disease activity score in patients with axial spondyloarthritis? Int J Rheum Dis 20(9):1201-1211

314. Huscher D et al (2015) Trends in treatment and outcomes of ankylosing spondylitis in outpatient rheumatological care in Germany between 2000 and 2012. RMD Open 1(1):e33

315. Doran MF et al (2003) Predictors of longterm outcome in ankylosing spondylitis. J Rheumatol 30(2):316-320

316. Amor B et al (1994) Predictive factors for the longterm outcome of spondyloarthropathies. JRheumatol 21(10):1883-1887
317. Gran JT, Skomsvoll JF (1997) The outcome of ankylosing spondylitis: a study of 100 patients. $\mathrm{Br}$ J Rheumatol 36(7):766-771

318. Averns HL et al (1996) Smoking and outcome in ankylosing spondylitis. Scand J Rheumatol 25(3):138-142

319. Ward MM (2002) Predictors of the progression of functional disability in patients with ankylosing spondylitis. JRheumatol 29(7):1420-1425

320. Brophy $S$ et al (2002) The natural history of ankylosing spondylitis as defined by radiological progression. JRheumatol 29(6):1236-1243

321. van der Heijde D et al (1997) Preliminary core sets for endpoints in ankylosing spondylitis. Assessments in Ankylosing Spondylitis Working Group. JRheumatol 24(11):2225-2229

322. van der Heijde D et al (1999) Ankylosing spondylitis: plenary discussion and results of voting on selection of domains and some specific instruments. JRheumatol 26(4):1003-1005

323. Anderson JJ et al (2001) Ankylosing spondylitis assessment group preliminary definition of shortterm improvement in ankylosing spondylitis. Arthritis Rheum 44(8):1876-1886

324. Sieper J et al (2012) Early response to adalimumab predicts long-term remission through 5 years of treatment in patients with ankylosing spondylitis. Ann Rheum Dis 71(5):700-706

325. Rudwaleit M et al (2010) Effectiveness and safety of adalimumab in patients with ankylosing spondylitis or psoriatic arthritis and history of antitumor necrosis factor therapy. Arthritis Res Ther 12(3):R117

326. Glintborg B et al (2010) Predictors of treatment response and drug continuation in 842 patients with ankylosing spondylitis treated with antitumour necrosis factor: results from 8 years' surveillance in the Danish nationwide DANBIO registry. Ann Rheum Dis 69(11):2002-2008

327. Lord PA et al (2010) Predictors of response to antiTNF therapy in ankylosing spondylitis: results from the British Society for Rheumatology Biologics Register. Rheumatol (oxford) 49(3):563-570

328. Perrotta FMetal (2014) Predictive factors for partial remission according to the Ankylosing Spondylitis Assessment Study working group in patients with ankylosing spondylitis treated with anti-TNFalpha drugs. Reumatismo 66(3):208-214

329. Rausch Osthoff AK et al (2018) EULAR recommendations for physical activity in people with inflammatory arthritis and osteoarthritis. Ann Rheum Dis 77(9):1251-1260

330. Klemz BN et al (2016) The relevance of performing exercise test before starting supervised physical exercise in asymptomatic cardiovascular patient with rheumatic diseases. Rheumatol (oxford) 55(11):1978-1986

331. Dagfinrud H, Kvien TK, Hagen KB (2008) Physiotherapy interventions for ankylosing spondylitis. Cochrane Database Syst Rev 2008(1):CD2822

332. van Tubergen A et al (2001) Combined spa-exercise therapy is effective in patients with ankylosing spondylitis: a randomized controlled trial. Arthritis Rheum 45(5):430-438

333. Escalas Cetal (2016) Evaluation of physiotherapy in a prospective cohort of early axial spondyloarthritis. Data from the DESIR cohort. Joint Bone Spine 83(2):185-190

334. Albrecht K, Huscher D (2017) Do we prescribe physical medicine sufficiently? Current data from the German Collaborative Arthritis Center. Akt Rheumatol 42:118-121

335. Martins NA et al (2014) Exercise and ankylosing spondylitis with New York modified criteria: a systematic review of controlled trials with metaanalysis. Acta Reumatol Port 39(4):298-308

336. Sharan D, Rajkumar JS (2017) Physiotherapy for Ankylosing Spondylitis: systematic review and a proposed rehabilitation protocol. Curr Rheumatol Rev 13(2):121-125

337. Jennings F et al (2015) Effects of aerobic training in patients with Ankylosing Spondylitis. J Rheumatol 42(12):2347-2353

338. Liang H et al (2015) Effects of home-based exercise intervention on health-related quality of life for patients with ankylosing spondylitis: a metaanalysis. Clin Rheumatol 34(10):1737-1744

339. Niedermann Ket al (2013) Effect of cardiovascular training on fitness and perceived disease activity in people with ankylosing spondylitis. Arthritis Care Res (hoboken) 65(11):1844-1852

340. Rosu MO et al (2014) Effects of Pilates, McKenzie and Heckscher training on disease activity, spinal motility and pulmonary function in patients with ankylosing spondylitis: a randomized controlled trial. Rheumatol Int 34(3):367-372

341. Altan $L$ et al (2012) Effect of Pilates training on people with ankylosing spondylitis. Rheumatol Int 32(7):2093-2099

342. Passalent LA et al (2010) Exercise in ankylosing spondylitis: discrepancies between recommendations and reality. J Rheumatol 37(4):835-841

343. Fongen C, Halvorsen S, Dagfinrud H (2013) High disease activity is related to low levels of physical activity in patients with ankylosing spondylitis. Clin Rheumatol 32(12):1719-1725

344. Kjeken let al (2013) A three-week multidisciplinary in-patient rehabilitation programme had positive long-term effects in patients with ankylosing spondylitis: randomized controlled trial. J Rehabil Med 45(3):260-267

345. Masiero $S$ et al (2014) Supervised training and home-based rehabilitation in patients with stabilized ankylosing spondylitis on TNF inhibitor treatment: a controlled clinical trial with a 12month follow-up. Clin Rehabil 28(6):562-572

346. Durmus D et al (2009) Effects of a home-based exercise program on quality of life, fatigue, and depression in patients with ankylosing spondylitis Rheumatol Int 29(6):673-677

347. Durmus D et al (2009) Effects of two exercise interventions on pulmonary functions in the patients with ankylosing spondylitis. Joint Bone Spine 76(2):150-155

348. Karapolat $\mathrm{H}$ et al (2009) Are swimming or aerobic exercise better than conventional exercise in ankylosing spondylitis patients? A randomized controlled study. Eur J Phys Rehabil Med 45(4):449-457

349. Widberg K, Karimi H, Hafstrom I (2009) Self- and manual mobilization improves spine mobility in men with ankylosing spondylitis-a randomized study. Clin Rehabil 23(7):599-608

350. Aytekin Eetal (2012) Home-based exercise therapy in patients with ankylosing spondylitis: effects on pain, mobility, disease activity, quality of life, and respiratory functions. Clin Rheumatol 31(1):91-97

351. Colina M et al (2009) Combination treatment with etanercept and an intensive spa rehabilitation program in active ankylosing spondylitis. Int J Immunopathol Pharmacol 22(4):1125-1129

352. Karahan AY et al (2016) The effectiveness of Exergamesin patients with Ankylosing Spondylitis: a randomized controlled trial. Adv Clin Exp Med 25(5):931-936

353. Kraag G et al (1990) The effects of comprehensive home physiotherapy and supervision on patients 
with ankylosing spondylitis - a randomized controlled trial. J Rheumatol 17(2):228-233

354. Ince $G$ et al (2006) Effects of a multimodal exercise program for people with ankylosing spondylitis. Phys Ther 86(7):924-935

355. Lim HJ, Moon Yl, Lee MS (2005) Effects of home-based daily exercise therapy on joint mobility, daily activity, pain, and depression in patients with ankylosing spondylitis. Rheumatol Int 25(3):225-229

356. Kraag $G$ et al (1994) The effects of comprehensive home physiotherapy and supervision on patients with ankylosing spondylitis-an 8-month followup. JRheumatol 21(2):261-263

357. Karapolat $\mathrm{H}$ et al (2008) Comparison of groupbased exercise versus home-based exercise in patients with ankylosing spondylitis: effects on Bath Ankylosing Spondylitis Indices, quality of life and depression. Clin Rheumatol 27(6):695-700

358. Hidding A et al (1993) Is group physical therapy superior to individualized therapy in ankylosing spondylitis? A randomized controlled trial. Arthritis Care Res 6(3):117-125

359. Helliwell P (1996) A randomised trial of three different physiotherapy regimes in ankylosing spondylitis. Physiotherapy 82:85-90

360. Analay Y et al (2003) The effectiveness of intensive group exercise on patients with ankylosing spondylitis. Clin Rehabil 17(6):631-636

361. Bakker C et al (1994) Cost effectiveness of group physical therapy compared to individualized therapy for ankylosing spondylitis. A randomized controlled trial. J Rheumatol 21(2):264-268

362. Ciprian L et al (2013) The effects of combined spa therapy and rehabilitation on patients with ankylosing spondylitis being treated with TNF inhibitors. Rheumatol Int 33(1):241-245

363. Gurcay E et al (2008) Stanger bath therapy for ankylosing spondylitis: illusion or reality? Clin Rheumatol 27(7):913-917

364. Altan L et al (2006) The effect of balneotherapy on patients with ankylosing spondylitis. Scand J Rheumatol 35(4):283-289

365. Codish S et al (2005) Spa therapy for ankylosing spondylltis at the Dead Sea. Isr Med Assoc J 7(7):443-446

366. Rutherford SM, Nicolson CF, Crowther ER (2005) Symptomatic improvement in function and disease activity in a patient with ankylosing spondylitis utilizing a course of chiropractic therapy: a prospective case study. J Can Chiropr Assoc 49(2):81-91

367. Oosterveld FG et al (2009) Infrared sauna in patients with rheumatoid arthritis and ankylosing spondylitis. A pilot study showing good tolerance, short-term improvement of pain and stiffness, and a trend towards long-term beneficial effects. Clin Rheumatol 28(1):29-34

368. Tarner IH et al (2009) The effect of mild whole-body hyperthermia on systemic levels of TNF-alpha, IL-1beta, and IL- 6 in patients with ankylosing spondylitis. Clin Rheumatol 28(4):397-402

369. Samborski W et al (1992) Can thermal therapy of ankylosing spondylitis induce an activation of the disease? ZRheumatol 51(3):127-131

370. Gemignani Get al (1991) Transcutaneous electrical nerve stimulation in ankylosing spondylitis: a double-blind study. Arthritis Rheum 34(6):788-789

371. Turan Y et al (2014) Is magnetotherapy applied to bilateral hips effective in ankylosing spondylitis patients? A randomized, double-blind, controlled study. Rheumatol Int 34(3):357-365

372. Silte Karamanlioglu D et al (2016) Effectiveness of ultrasound treatment applied with exercise therapy on patients with ankylosing spondylitis: a double-blind, randomized, placebo-controlled trial. Rheumatol Int 36(5):653-661

373. Spadaro A et al (2008) Occupational therapy in ankylosing spondylitis: Short-term prospective study in patients treated with anti-TNF-alpha drugs. Joint Bone Spine 75(1):29-33

374. Barkhuizen A et al (2006) Celecoxib is efficacious and well tolerated in treating signs and symptoms of ankylosing spondylitis. J Rheumatol 33(9):1805-1812

375. Dougados M et al (1989) A double blind crossove placebo controlled trial of ximoprofen in as JRheumatol 16(8):1167-1169

376. Dougados Met al (1994) Ximoprofen in ankylosing spondylitis. Adoubleblind placebocontrolleddose ranging study. Scand J Rheumatol 23(5):243-248

377. Sturrock RD, Hart FD (1974) Double-blind crossover comparison of indomethacin, flurbiprofen, and placebo in ankylosing spondylitis. Ann Rheum Dis 33(2):129-131

378. van der Heijde D et al (2005) Evaluation of the efficacy of etoricoxib in ankylosing spondylitis: results of a fifty-two-week, randomized, controlled study. Arthritis Rheum 52(4):1205-1215

379. Kroon FP et al (2016) Nonsteroidal antiinflammatory drugs for axial Spondyloarthritis: a Cochrane review. JRheumatol 43(3):607-617

380. Wang R, Dasgupta A, Ward MM (2016) Comparative efficacy of non-steroidal anti-inflammatory drugs in ankylosing spondylitis: a Bayesian network meta-analysis of clinical trials. Ann Rheum Dis 75(6):1152-1160

381. Sieper J et al (2014) Efficacy and safety of infliximab plus naproxen versus naproxen alone in patients with early, active axial spondyloarthritis: results from the double-blind, placebo-controlled INFAST study, Part 1. Ann Rheum Dis 73(1):101-107

382. Gossec L et al (2005) Efficacy of cyclo-oxygenase2 inhibition by etoricoxib and naproxen on the axial manifestations of ankylosing spondylitis in the presence of peripheral arthritis. Ann Rheum Dis 64(11):1563-1567

383. Sieper J et al (2008) Comparison of two different dosages of celecoxib with diclofenac for the treatment of active ankylosing spondylitis: results of a 12-week randomised, double-blind, controlled study. Ann Rheum Dis 67(3):323-329

384. Escalas C, Trijau S, Dougados M (2010) Evaluation of the treatment effect of NSAIDs/TNF blockers according to different domains in ankylosing spondylitis: results of a meta-analysis. Rheumato (oxford) 49(7):1317-1325

385. Walker C et al (2016) Celecoxib versus diclofenac for the treatment of ankylosing spondylitis: 12 week randomized study in Norwegian patients. J IntMed Res 44(3):483-495

386. Balazcs E et al (2016) A randomized, clinical trial to assess the relative efficacy and tolerability of two doses of etoricoxib versus naproxen in patients with ankylosing spondylitis. Bmc Musculoskelet Disord 17(1):426

387. Peloso PM et al (2011) Longitudinal numbersneeded-to-treat (NNT) for achieving various level of analgesic response and improvement with etoricoxib, naproxen, and placebo in ankylosing spondylitis. Bmc Musculoskelet Disord 12:165

388. Dougados $M$ et al (2014) Evaluation of the nonsteroidal anti-inflammatory drug-sparing effect of etanercept in axial spondyloarthritis: results of the multicenter, randomized, doubleblind, placebo-controlled SPARSE study. Arthritis Res Ther 16(6):481
389. van der Heijde D et al (2011) 2010 Update of the international ASAS recommendations for the use of anti-TNF agents in patients with axial spondyloarthritis. Ann Rheum Dis 70(6):905-908

390. Zochling $J$ et al (2006) Nonsteroidal anti-inflammatory drug use in ankylosing spondylitis-a population-based survey. Clin Rheumatol 25(6):794-800

391. Wanders A et al (2005) Nonsteroidal antiinflammatory drugs reduce radiographic progression in patients with ankylosing spondylitis:arandomized clinical trial. Arthritis Rheum 52(6):1756-1765

392. Sieper J et al (2016) Effect of continuous versus on-demand treatment of ankylosing spondylitis with diclofenac over 2 years on radiographic progression of the spine: results from a randomised multicentre trial (ENRADAS). Ann Rheum Dis 75(8):1438-1443

393. Colebatch AN, Marks JL, Edwards CJ (2011) Safety of non-steroidal anti-inflammatory drugs, including aspirin and paracetamol (acetaminophen) in people receiving methotrexate for inflammatory arthritis (rheumatoid arthritis, ankylosing spondylitis, psoriatic arthritis, other spondyloarthritis). Cochrane Database Syst Rev 2011(11):CD8872

394. Kristensen LE et al (2015) Safety of Etoricoxib, Celecoxib, and nonselective Nonsteroidal antiinflammatory drugs in Ankylosing Spondylitis and other Spondyloarthritis patients: a Swedish national population-based cohort study. Arthritis Care Res (hoboken) 67(8):1137-1149

395. Cannon CP et al (2006) Cardiovascular outcomes with etoricoxib and diclofenac in patients with osteoarthritis and rheumatoid arthritis in the Multinational Etoricoxib and Diclofenac Arthritis Long-term (MEDAL) programme: a randomised comparison. Lancet 368(9549):1771-1781

396. Farkouh ME et al (2004) Comparison of lumiracoxib with naproxen and ibuprofen in the Therapeutic Arthritis Research and Gastrointestinal Event Trial (TARGET), cardiovascular outcomes: randomised controlled trial. Lancet 364(9435):675-684

397. Song IH et al (2008) Benefits and risks of ankylosing spondylitis treatment with nonsteroidal antiinflammatory drugs. Arthritis Rheum 58(4):929-938

398. Bally M et al (2017) Risk of acute myocardial infarction with NSAIDs in real world use: bayesian meta-analysis of individual patient data. BMJ 357:j1909

399. Fanelli A et al (2017) Cardiovascular and cerebrovascular risk with nonsteroidal anti-inflammatory drugs and cyclooxygenase 2 inhibitors: latest evidence and clinical implications. Ther Adv Drug Saf8(6):173-182

400. Bolten W, Krüger K, Reiter S (2009) Nichtsteroidale Antirheumatika, aktualisierte Empfehlungen zur Verordnung. Deutsche Gesellschaft für Rheumatologie. https://dgrh.de/dam/ jcr:4a4ce23b-d97e-4395-b091-6368d9e8c7d4/ Nichtsteroidale_Antirheumatika_2009_final.pd

401. Burmester $G$ et al (2011) The appropriate use of non-steroidal anti-inflammatory drugs in rheumatic disease: opinions of a multidisciplinary European expert panel. Ann Rheum Dis 70(5):818-822

402. Arzneimittelkommission der deutschen Ärzteschaft (2008) Empfehlungen zur Therapie von degenerativen Gelenkerkrankungen. 35(Sonderheft 1)

403. Lanza FL et al (2009) Guidelines for prevention of NSAID-related ulcer complications. Am J Gastroenterol 104(3):728-738 
404. Yokoyama Het al (2013) Influence of non-steroidal anti-inflammatory drugs on antiplatelet effect of aspirin. JClin Pharm Ther 38(1):12-15

405. Catella-Lawson F et al (2001) Cyclooxygenase inhibitors and the antiplatelet effects of aspirin NEngl J Med 345(25):1809-1817

406. Zhang $X$ et al (2017) Non-steroidal anti-inflammatory drug induced acute kidney injury in the community dwelling general population and people with chronic kidney disease: systematic review and meta-analysis. Bmc Nephrol 18(1):256

407. Gooch K et al (2007) NSAID use and progression of chronic kidney disease. Am J Med 120(3):280 e1-280 e7

408. Basavaraj KH et al (2010) The role of drugs in the induction and/or exacerbation of psoriasis. Int J Dermatol 49(12):1351-1361

409. Gossec L et al (2016) European League Against Rheumatism (EULAR) recommendations for the management of psoriatic arthritis with pharmacological therapies: 2015 update. Ann Rheum Dis 75(3):499-510

410. Canoui-Poitrine F et al (2014) Early tumor necrosis factor alpha antagonist therapy in everyday practice for inflammatory back pain suggesting axial spondyloarthritis: results from a prospective multicenter french cohort. Arthritis Care Res (hoboken) 66(9):1395-1402

411. Chen C et al (2016) Comparative effectiveness of biologic therapy regimens for Ankylosing Spondylitis: a systematic review and a network Meta-analysis. Medicine (Baltimore) 95(11):e3060

412. Betts KA et al (2016) Network Meta-analysis and cost Per responder of tumor necrosis factor-alpha and Interleukin inhibitors in the treatment of active Ankylosing Spondylitis. Rheumatol Ther 3(2):323-336

413. Callhoff J et al (2015) Efficacy of TNFalpha blockers in patients with ankylosing spondylitis and nonradiographic axial spondyloarthritis: a metaanalysis. Ann Rheum Dis 74(6):1241-1248

414. Li ZH et al (2013) Etanercept in the treatment of ankylosing spondylitis: a meta-analysis of randomized, double-blind, placebo-controlled clinical trials, and the comparison of the Caucasian and Chinese population. Eur J Orthop Surg Traumatol 23(5):497-506

415. Wang $H$ et al (2014) Randomized, placebo controlled and double-blind trials of efficacy and safety of adalimumab for treating ankylosing spondylitis: a meta-analysis. Int J Rheum Dis 17(2):142-148

416. Wang $Y$ et al (2016) Comparative efficacy and acceptability of anti-TNF-Alpha therapy in Ankylosing Spondylitis: a mixed-treatments comparison. Cell Physiol Biochem 39(5):1679-1694

417. Braun Jet al (1995) Use of immunohistologic and in situ hybridization techniques in the examination of sacroiliac joint biopsy specimens from patients with ankylosing spondylitis. Arthritis Rheum 38(4):499-505

418. Zochling J et al (2006) Current evidence for the management of ankylosing spondylitis: a systematic literature review for the ASAS/EULAR management recommendations in ankylosing spondylitis. Ann Rheum Dis 65(4):423-432

419. Sepriano A et al (2017) Efficacy and safety of biological and targeted-synthetic DMARDs: a systematic literature review informing the 2016 update of the ASAS/EULAR recommendations for the management of axial spondyloarthritis. RMD Open 3(1):e396
420. Maxwell LJ et al (2015) TNF-alpha inhibitors for ankylosing spondylitis. Cochrane Database Syst Rev 2015(4):CD5468

421. Olivieri I et al (2016) Efficacy, safety and cost per responder of biologics in the treatment of non-radiographic axial spondyloarthritis. Clin Exp Rheumatol 34(5):935-940

422. Ren $L$ et al (2013) Efficacy of antitumor necrosis factor(alpha) agents on patients with ankylosing spondylitis. Am J Med Sci 346(6):455-461

423. van der Heijde D et al (2008) Adalimumab effectively reduces the signs and symptoms of active ankylosing spondylitis in patients with tota spinal ankylosis. Ann Rheum Dis 67(9):1218-1221

424. Dougados M et al (2011) Efficacy of etanercept on rheumatic signs and pulmonary function tests in advanced ankylosing spondylitis: results of a randomised double-blind placebo-controlled study (SPINE). Ann Rheum Dis 70(5):799-804

425. Haibel $\mathrm{H}$ et al (2008) Efficacy of adalimumab in the treatment of axial spondylarthritis without radiographically defined sacroiliitis: results of a twelve-week randomized, double-blind, placebo-controlled trial followed by an open-label extension up to week fifty-two. Arthritis Rheum 58(7):1981-1991

426. Song IH et al (2011) Effects of etanercept versus sulfasalazine in early axial spondyloarthritis on active inflammatory lesions as detected by whole body MRI (ESTHER): a 48-week randomised controlled trial. Ann Rheum Dis 70(4):590-596

427. Barkham N et al (2009) Clinical and imaging efficacy of infliximab in HLA-B27-Positive patients with magnetic resonance imaging-determined early sacroiliitis. Arthritis Rheum 60(4):946-954

428. Sieper J et al (2013) Efficacy and safety of adalimumab in patients with non-radiographic axial spondyloarthritis: results of a randomised placebo-controlled trial (ABILITY-1). Ann Rheum Dis 72(6):815-822

429. CorbettMetal(2016)Tumournecrosisfactor-alph inhibitors for ankylosing spondylitis and nonradiographic axial spondyloarthritis: a systematic review and economic evaluation. Health Technol Assess 20(9):1-334

430. Ciurea A et al (2013) Tumor necrosis factor alpha inhibition in radiographic and Nonradiographic axial Spondyloarthritis: results from a large observational cohort. Arthritis Rheum 65(12):3096-3106

431. Corli J et al (2015) Tumor necrosis factor-alpha inhibition in Ankylosing Spondylitis and Nonradiographic axial Spondyloarthritis: treatment response, drug survival, and patient outcome. JRheumatol 42(12):2376-2382

432. Kristensen LE et al (2010) Presence of periphera arthritis and male sex predicting continuation of anti-tumor necrosis factor therapy in ankylosing spondylitis: an observational prospective cohort study from the South Swedish Arthritis Treatment Group Register. Arthritis Care Res (hoboken) 62(10):1362-1369

433. Arends S et al (2011) Baseline predictors of response and discontinuation of tumor necrosis factor-alpha blocking therapy in ankylosing spondylitis: a prospective longitudinal observational cohort study. Arthritis Res Ther 13(3):R94

434. Vastesaeger $\mathrm{N}$ et al (2011) Predicting the outcome of ankylosing spondylitis therapy. Ann Rheum Dis 70(6):973-981

435. Maneiro JR et al (2015) Predictors of response to TNF antagonists in patients with ankylosing spondylitis and psoriatic arthritis: systematic review and meta-analysis. RMD Open 1(1):e17
436. van der Heijde D et al (2006) Efficacy and safety of adalimumab in patients with ankylosing spondylitis: results of a multicenter, randomized, double-blind, placebo-controlled trial. Arthritis Rheum 54(7):2136-2146

437. van der Heijde D et al (2009) Adalimumab effectiveness for the treatment of ankylosing spondylitis is maintained for up to 2 years: longterm results from the ATLAS trial. Ann Rheum Dis 68(6):922-929

438. Davis JC Jr. et al (2003) Recombinant human tumor necrosis factor receptor (etanercept) for treating ankylosing spondylitis: a randomized, controlled trial. Arthritis Rheum 48(11):3230-3236

439. Davis JC et al (2005) Sustained durability and tolerability of etanercept in ankylosing spondylitis for 96 weeks. Ann Rheum Dis 64(11):1557-1562

440. Davis JC Jr. et al (2008) Efficacy and safety of up to 192 weeks of etanercept therapy in patients with ankylosing spondylitis. Ann Rheum Dis 67(3):346-352

441. Calin A et al (2004) Outcomes of a multicentre randomised clinical trial of etanercept to treat ankylosing spondylitis. Ann Rheum Dis 63(12):1594-1600

442. Dijkmans B et al (2009) Etanercept in the longterm treatment of patients with ankylosing spondylitis. JRheumatol 36(6):1256-1264

443. Martin-Mola E et al (2010) Sustained efficacy and safety, including patient-reported outcomes, with etanercept treatment over 5 years in patients with ankylosing spondylitis. Clin Exp Rheumatol 28(2):238-245

444. van der Heijde D et al (2006) Etanercept $50 \mathrm{mg}$ once weekly is as effective as $25 \mathrm{mg}$ twice weekly in patients with ankylosing spondylitis. Ann Rheum Dis 65(12):1572-1577

445. Dougados $\mathrm{M}$ et al (2010) A randomised, multicentre, double-blind, placebo-controlled trial of etanercept in adults with refractory heel enthesitis in spondyloarthritis: the HEEL trial. Ann Rheum Dis 69(8):1430-1435

446. Navarro-Sarabia F et al (2011) High-dose etanercept in ankylosing spondylitis: results of a 12week randomized, double blind, controlled multicentre study (LOADET study). Rheumatol (oxford) 50(10):1828-1837

447. Braun J et al (2011) Clinical efficacy and safety of etanercept versus sulfasalazine in patients with ankylosing spondylitis: a randomized, doubleblind trial. Arthritis Rheum 63(6):1543-1551

448. Inman RD et al (2008) Efficacy and safety of golimumab in patients with ankylosing spondylitis: results of a randomized, double-blind, placebo-controlled, phase III trial. Arthritis Rheum 58(11):3402-3412

449. Braun J et al (2002) Treatment of active ankylosing spondylitis with infliximab: a randomised controlled multicentre trial. Lancet 359(9313):1187-1193

450. Braun J et al (2005) Two year maintenance of efficacy and safety of infliximab in the treatment of ankylosing spondylitis. Ann Rheum Dis 64(2):229-234

451. Braun J et al (2005) Persistent clinical response to the anti-TNF-alpha antibody infliximab in patients with ankylosing spondylitis over 3 years. Rheumatol (oxford) 44(5):670-676

452. Braun J et al (2008) Persistent clinical efficacy and safety of anti-tumour necrosis factor alpha therapy with infliximab in patients with ankylosing spondylitis over 5 years: evidence for different types of response. Ann Rheum Dis 67(3):340-345 
453. Baraliakos $X$ et al (2011) Persistent clinical efficacy and safety of infliximab in ankylosing spondylitis after 8 years - early clinical response predicts long-term outcome. Rheumatol (oxford) 50(9):1690-1699

454. van der Heijde D et al (2005) Efficacy and safety of infliximab in patients with ankylosing spondylitis: results of a randomized, placebo-controlled trial (ASSERT). Arthritis Rheum 52(2):582-591

455. Heldmann F et al (2011) The European ankylosing spondylitis infliximab cohort (EASIC): a European multicentre study of long term outcomes in patients with ankylosing spondylitis treated with infliximab. Clin Exp Rheumatol 29(4):672-680

456. Bao Cet al (2014) Safety and efficacy of golimumab in Chinese patients with active ankylosing spondylitis: 1-year results of a multicentre randomized, double-blind, placebo-controlled phase III trial. Rheumatol (oxford) 53(9):1654-1663

457. Deodhar A et al (2018) Safety and efficacy of Golimumab administered intravenously in adults with Ankylosing Spondylitis: results through week 28 of the GO-ALIVE study. J Rheumatol 45(3):341-348

458. Dougados M et al (2014) Symptomatic efficacy of etanercept and its effects on objective signs of inflammation in early nonradiographic axial spondyloarthritis: a multicenter, randomized, double-blind, placebo-controlled trial. Arthritis Rheumatol 66(8):2091-2102

459. Huang $F$ et al (2014) Efficacy and safety of adalimumab in Chinese adults with active ankylosing spondylitis: results of a randomised, controlled trial. Ann Rheum Dis 73(3):587-594

460. Landewe R et al (2014) Efficacy of certolizumab pegol on signs and symptoms of axial spondyloarthritis including ankylosing spondylitis: 24-week results of a double-blind randomised placebo-controlled Phase 3 study. Ann Rheum Dis 73(1):39-47

461. Park W et al (2013) A randomised, double-blind, multicentre, parallel-group, prospective study comparing the pharmacokinetics, safety, and efficacy of CT-P13 and innovator infliximab in patients with ankylosing spondylitis: the PLANETAS study. Ann Rheum Dis 72(10):1605-1612

462. Cohen JD et al (2007) Psoriasis induced by tumor necrosis factor-alpha antagonist therapy: a case series. J Rheumatol 34(2):380-385

463. Davis JC Jr. et al (2007) Health-related quality of life outcomes in patients with active ankylosing spondylitis treated with adalimumab: results from a randomized controlled study. Arthritis Rheum 57(6):1050-1057

464. Braun J et al (2007) Improvement in patientreported outcomes for patients with ankylosing spondylitis treated with etanercept $50 \mathrm{mg}$ onceweekly and $25 \mathrm{mg}$ twice-weekly. Rheumatol (oxford) 46(6):999-1004

465. Boonen A et al (2008) Rapid and sustained improvement in health-related quality of life and utility for 72 weeks in patients with ankylosing spondylitis receiving etanercept. J Rheumatol 35(4):662-667

466. Kimel M et al (2011) Norms-based assessment of patient-reported outcomes associated with adalimumab monotherapy in patients with ankylosing spondylitis. Clin Exp Rheumatol 29(4):624-632

467. Braun Jetal (2009) Improvement in hemoglobin levels in patients with ankylosing spondylitis treated with infliximab. Arthritis Rheum 61(8):1032-1036

468. Gonnet-Gracia C et al (2008) Anti-nuclear antibodies, anti-DNA and C4 complement evolution in rheumatoid arthritis and ankylosing spondylitis treated with TNF-alpha blockers. Clin Exp Rheumatol 26(3):401-407

469. Maksymowych WP et al (2010) Low-dose infliximab $(3 \mathrm{mg} / \mathrm{kg})$ significantly reduces spinal inflammation on magnetic resonance imaging in patients with ankylosing spondylitis: a randomized placebo-controlled study. J Rheumatol 37(8):1728-1734

470. van der Heijde D et al (2008) Radiographic progression of ankylosing spondylitis after up to two years of treatment with etanercept. Arthritis Rheum 58(5):1324-1331

471. Baraliakos X et al (2007) Radiographic progression in patients with ankylosing spondylitis after 4 yrs of treatment with the anti-TNF-alpha antibody infliximab. Rheumatol (oxford) 46(9):1450-1453

472. Haroon $N$ et al (2013) The impact of tumor necrosis factor alpha inhibitors on radiographic progression in ankylosing spondylitis. Arthritis Rheum 65(10):2645-2654

473. Kim TJ et al (2016) Radiographic progression in patients with ankylosing spondylitis according to tumor necrosis factor blocker exposure: Observation Study of Korean Spondyloarthropathy Regis try (OSKAR) data. Joint Bone Spine 83(5):569-572

474. MaasFetal (2017) Reduction in spinal radiographic progression in Ankylosing Spondylitis patients receiving prolonged treatment with tumor necrosis factor inhibitors. Arthritis Care Res (hoboken) 69(7):1011-1019

475. Molnar C et al (2018) TNF blockers inhibit spinal radiographic progression in ankylosing spondylitis by reducing disease activity: results from the Swiss Clinical Quality Management cohort. Ann Rheum Dis 77(1):63-69

476. Park JW et al (2016) Impact of dose tapering of tumor necrosis factor inhibitor on radiographic progression in Ankylosing Spondylitis. PLoS ONE 11(12):e168958

477. Visvanathan S et al (2009) Effects of infliximab on markers of inflammation and bone turnover and associations with bone mineral density in patients with ankylosing spondylitis. Ann Rheum Dis 68(2):175-182

478. Siu S et al (2015) Meta-analysis of tumor necrosis factor inhibitors and glucocorticoids on bone density in rheumatoid arthritis and ankylosing spondylitis trials. Arthritis Care Res (hoboken) 67(6):754-764

479. van der Heijde D et al (2006) Infliximab improves productivity and reduces workday loss in patients with ankylosing spondylitis: results from a randomized, placebo-controlled trial. Arthritis Rheum 55(4):569-574

480. Barkham N et al (2010) Double-blind placebocontrolled trial of etanercept in the prevention of work disability in ankylosing spondylitis. Ann Rheum Dis 69(11):1926-1928

481. Keat AC et al (2008) Influence of biologic therapy on return to work in people with work disability due to ankylosing spondylitis. Rheumatol (oxford) 47(4):481-483

482. Kristensen LE et al (2012) Sick leave in patients with ankylosing spondylitis before and after antiTNF therapy: a population-based cohort study. Rheumatology (Oxford) 51(2):243-249

483. Maksymowych WP et al (2010) Impact of age, sex, physical function, health-related quality of life, and treatment with adalimumab on work status and work productivity of patients with ankylosing spondylitis. JRheumatol 37(2):385-392

484. Wallman JK et al (2017) Work disability in non-radiographic axial spondyloarthritis patients before and after start of anti-TNF therapy: a population-based regional cohort study from southern Sweden. Rheumatology (Oxford) 56(5):716-724

485. Baeten D et al (2013) Anti-interleukin-17A monoclonal antibody secukinumab in treatment of ankylosing spondylitis: a randomised, double-blind, placebo-controlled trial. Lancet 382(9906):1705-1713

486. Baeten Det al (2015) Secukinumab, an Interleukin$17 \mathrm{~A}$ Inhibitor, in Ankylosing Spondylitis. N Engl J Med373(26):2534-2548

487. Sieper J et al (2017) Secukinumab efficacy in anti-TNF-naive and anti-TNF-experienced subjects with active ankylosing spondylitis: results from the MEASURE 2 Study. Ann Rheum Dis 76(3):571-592

488. Pavelka K et al (2017) Efficacy, safety, and tolerability of secukinumab in patients with active ankylosing spondylitis: a randomized, doubleblind phase 3 study, MEASURE 3 . Arthritis Res Ther 19(1):285

489. Deodhar AA et al (2016) Effect of Secukinumab on patient-reported outcomes in patients with active Ankylosing Spondylitis: a phase III randomized trial (MEASURE 1). Arthritis Rheumatol 68(12):2901-2910

490. Deodhar A et al (2019) Secukinumab provides rapid and persistent relief in pain and fatigue symptoms in patients with ankylosing spondylitis irrespective of baseline C-reactive protein levels or prior tumour necrosis factor inhibitor therapy: 2-year data from the MEASURE 2 study. Clin Exp Rheumatol 37(2):260-269

491. Baraliakos X et al (2016) Long-term effects of secukinumab on MRI findings in relation to clinical efficacy in subjects with active ankylosing spondylitis: an observational study. Ann Rheum Dis 75(2):408-412

492. Baraliakos X et al (2018) Long-term effects of interleukin-17A inhibition with secukinumab in active ankylosing spondylitis: 3-year efficacy and safety results from an extension of the Phase 3 MEASURE 1 trial. Clin Exp Rheumatol 36(1):50-55

493. Braun J et al (2017) Effect of secukinumab on clinical and radiographic outcomes in ankylosing spondylitis: 2-year results from the randomised phase III MEASURE 1 study. Ann Rheum Dis 76(6):1070-1077

494. Marzo-Ortega $H$ et al (2017) Secukinumab provides sustained improvements in the signs and symptoms of active ankylosing spondylitis with high retention rate: 3 -year results from the phase III trial, MEASURE 2. RMD Open 3(2):e592

495. Song IH etal (2010) Different response to rituximab in tumor necrosis factor blocker-naive patients with active ankylosing spondylitis and in patients in whom tumor necrosis factor blockers have failed: a twenty-four-week clinical trial. Arthritis Rheum 62(5):1290-1297

496. Song IH et al (2011) Treatment of active ankylosing spondylitis with abatacept: an open-label, 24week pilot study. Ann Rheum Dis 70(6):1108-1110

497. Sieper J et al (2015) Sarilumab for the treatment of ankylosing spondylitis: results of a Phase II, randomised, double-blind, placebo-controlled study (ALIGN). Ann Rheum Dis 74(6):1051-1057

498. Sieper J et al (2014) Assessment of short-term symptomatic efficacy of tocilizumab in ankylosing spondylitis: results of randomised, placebocontrolled trials. Ann Rheum Dis 73(19):95-100

499. Poddubnyy D et al (2014) Ustekinumab for the treatment of patients with active ankylosing spondylitis: results of a 28-week, prospective, open-label, proof-of-concept study (TOPAS). Ann Rheum Dis 73(5):817-823 
500. Deodhar A et al (2019) Three Multicenter randomized, double-blind, placebo-controlled studies evaluating the efficacy and safety of Ustekinumab in axial Spondyloarthritis. Arthritis Rheumatol 71(2):258-270

501. Heiberg MS et al (2008) The comparative one-year performance of anti-tumor necrosis factor alpha drugs in patients with rheumatoid arthritis, psoriatic arthritis, and ankylosing spondylitis: results from a longitudinal, observational, multicenter study. Arthritis Rheum 59(2):234-240

502. Glintborg B et al (2013) Clinical response, drug survival and predictors thereof in 432 ankylosing spondylitis patients after switching tumour necrosis factor alpha inhibitor therapy: results from the Danish nationwide DANBIO registry. Ann Rheum Dis 72(7):1149-1155

503. Ciurea A et al (2016) Impaired response to treatment with tumour necrosis factor alpha inhibitors in smokers with axial spondyloarthritis. Ann Rheum Dis 75(3):532-539

504. Glintborg B et al (2016) Impact of tobacco smoking on response to tumour necrosis factoralpha inhibitor treatment in patients with ankylosing spondylitis: results from the Danish nationwide DANBIO registry. Rheumatol (oxford) 55(4):659-668

505. Micheroli R et al (2017) Impact of obesity on the response to tumornecrosisfactorinhibitors in axial spondyloarthritis. Arthritis Res Ther 19(1):164

506. Breban M et al (2008) Maintenance of infliximab treatment in ankylosing spondylitis: results of a one-year randomized controlled trial comparing systematic versus on-demand treatment. Arthritis Rheum 58(1):88-97

507. Marzo-Ortega $\mathrm{H}$ et al (2005) Infliximab in combination with methotrexate in active ankylosing spondylitis: a clinical and imaging study. Ann Rheum Dis 64(11):1568-1575

508. Li EK et al (2008) Short-term efficacy of combination methotrexate and infliximab in patients with ankylosing spondylitis: a clinical and magnetic resonance imaging correlation. Rheumatol (oxford) 47(9):1358-1363

509. Perez-Guijo VC et al (2007) Increased efficacy of infliximab associated with methotrexate in ankylosing spondylitis. Joint Bone Spine 74(3):254-258

510. Ma Z et al (2017) Safety of tumor necrosis factoralpha inhibitors for treatment of ankylosing spondylitis: a meta-analysis. Medicine (Baltimore) 96(25):e7145

511. Diel R et al (2009) Recommendations for tuberculosis screening before initiation of TNFalpha-inhibitor treatment in rheumatic diseases. Pneumologie 63(6):329-334

512. Fouque-Aubert $A$ et al (2010) Serious infections in patients with ankylosing spondylitis with and without TNF blockers: a systematic review and meta-analysis of randomised placebo-controlled trials. Ann Rheum Dis 69(10):1756-1761

513. Xu Z et al (2017) Risk of infection in patients with spondyloarthritis and ankylosing spondylitis receiving antitumor necrosis factor therapy: a meta-analysis of randomized controlled trials. Exp Ther Med 14(4):3491-3500

514. Mariette $X$ et al (2011) Malignancies associated with tumour necrosis factor inhibitors in registries and prospective observational studies: a systematic review and meta-analysis. Ann Rheum Dis 70(11):1895-1904

515. Baraliakos $X$ et al (2005) Clinical response to discontinuation of anti-TNF therapy in patients with ankylosing spondylitis after 3 years of continuous treatment with infliximab. Arthritis Res Ther 7(3):R439-R444

516. Landewe R et al (2018) Efficacy and safety of continuing versus withdrawing adalimumab therapy in maintaining remission in patients with non-radiographic axial spondyloarthritis (ABILITY 3): a multicentre, randomised, double-blind study Lancet 392(10142):134-144

517. Sieper J et al (2014) Maintenance of biologicfree remission with naproxen or no treatment in patients with early, active axial spondyloarthritis: results from a 6-month, randomised, open-label follow-up study, INFAST Part 2. Ann Rheum Dis 73(1):108-113

518. Fong W et al (2016) The effectiveness of a real life dose reduction strategy for tumour necrosis facto inhibitors in ankylosing spondylitis and psoriatic arthritis. Rheumatol (oxford) 55(10):1837-1842

519. Inman RD, Maksymowych WP (2010) A doubleblind, placebo-controlled trial of low dose infliximab in ankylosing spondylitis. J Rheumato 37(6):1203-1210

520. Krzysiek R et al (2009) Circulating concentration of infliximab and response to treatment in ankylosing spondylitis: results from a randomized control study. Arthritis Rheum 61(5):569-576

521. Arends $S$ et al (2010) The formation of autoantibodies and antibodies to TNF-alpha blocking agents in relation to clinical response in patients with ankylosing spondylitis. Clin Exp Rheumatol 28(5):661-668

522. de Vries MKetal (2007) Decreased clinical response to infliximab in ankylosing spondylitis is correlated with anti-infliximab formation. Ann Rheum Dis 66(9):1252-1254

523. de Vries MK et al (2009) Immunogenicity does not influence treatment with etanercept in patients with ankylosing spondylitis. Ann Rheum Dis 68(4):531-535

524. Lie E et al (2011) Effectiveness of switching between TNF inhibitors in ankylosing spondylitis: data from the NOR-DMARD register. Ann Rheum Dis 70(1):157-163

525. Ciurea A et al (2016) Does the reason for discontinuation of a first TNF inhibitor influence the effectiveness of a second TNF inhibitor in axial spondyloarthritis? Results from the Swiss Clinical Quality Management Cohort. Arthritis Res Ther 18:71

526. Navarro-Compan V et al (2017) Switching biological disease-modifying antirheumatic drugs in patients with axial spondyloarthritis: results from a systematic literature review. RMDOpen 3(2):e524

527. Deodhar A, Yu D (2017) Switching tumor necrosis factor inhibitors in the treatment of axial spondyloarthritis. Semin Arthritis Rheum 47(3):343-350

528. Sepriano A et al (2016) Effect of Comedication with conventional synthetic disease-modifying Antirheumatic drugs on retention of tumor necrosis factor inhibitors in patients with Spondyloarthritis: a prospective cohort study. Arthritis Rheumatol 68(11):2671-2679

529. Nissen MJ et al (2016) The effect of Comedication with a conventional synthetic disease-modifying Antirheumatic drug on drug retention and clinical effectiveness of anti-tumor necrosis factor therapy in patients with axial Spondyloarthritis. Arthritis Rheumatol 68(9):2141-2150

530. Chen J, Liu C (2005) Sulfasalazine for ankylosing spondylitis. Cochrane Database Syst Rev 2005(2):CD4800
531. Chen J, Lin S, Liu C (2014) Sulfasalazine for ankylosing spondylitis. Cochrane Database Syst Rev 2014(11):CD4800

532. Fagerli KM et al (2014) Is there a role for sulphasalazine in axial spondyloarthritis in the era of TNF inhibition? Data from the NOR-DMARD longitudinal observational study. Rheumatol (oxford) 53(6):1087-1094

533. Genc $\mathrm{H}$ et al (2007) The effects of sulfasalazine treatment on enthesal abnormalities of inflammatory rheumatic diseases. Clin Rheumatol 26(7):1104-1110

534. Braun J et al (2006) Efficacy of sulfasalazine in patients with inflammatory back pain due to undifferentiated spondyloarthritis and early ankylosing spondylitis: a multicentre randomised controlled trial. Ann Rheum Dis 65(9):1147-1153

535. Khanna Sharma S et al (2018) A randomized controlled trial to study the efficacy of sulfasalazine for axial disease in ankylosing spondylitis. Int J Rheum Dis 21(1):308-314

536. Liu YF et al (2014) Etanercept in the treatment of ankylosing spondylitis: a systematic review and meta-analysis. Exp Ther Med 8(5):1585-1592

537. Deng $X$ et al (2013) Thalidomide reduces recurrence of ankylosing spondylitis in patients following discontinuation of etanercept. Rheumatol Int 33(6):1409-1413

538. Chen J, Liu C, Lin J (2006) Methotrexate for ankylosing spondylitis. Cochrane Database Syst Rev 2006(4):CD4524

539. Chen J et al (2013) Methotrexate for ankylosing spondylitis. Cochrane Database Syst Rev CD004524:2

540. Haibel Het al (2014) Efficacy of oral prednisolone in active ankylosing spondylitis: results of a doubleblind, randomised, placebo-controlled short-term trial. Ann Rheum Dis 73(1):243-246

541. Maksymowych WP et al (2002) A six-month randomized, controlled, double-blind, dose-response comparison of intravenous pamidronate $(60 \mathrm{mg}$ versus $10 \mathrm{mg}$ ) in the treatment of nonsteroidal antiinflammatory drug-refractory ankylosing spondylitis. Arthritis Rheum 46(3):766-773

542. Haibel Hetal (2003) Treatment of active ankylosing spondylitis with pamidronate. Rheumatol (oxford) 42(8):1018-1020

543. Cairns AP et al (2005) An open study of pulse pamidronate treatment in severe ankylosing spondylitis, and its effect on biochemical markers of bone turnover. Ann Rheum Dis 64(2):338-339

544. Malaviya AN et al (2007) A new strategy of drug treatment in NSAID-unresponsive ankylosing spondylitis: combination of pamidronate and methylprednisolone monthly intravenous infusions on the background of a combination of disease modifying drugs sulfasalazine and methotrexate. JAssocPhysicians India 55:193-197

545. Santra G et al (2010) Assessment of the efficacy of pamidronate in ankylosing spondylitis: an open prospective trial. Singapore Med J 51(11):883-887

546. Coates L et al (2017) Clinical efficacy of oral alendronate in ankylosing spondylitis: a randomised placebo-controlled trial. Clin Exp Rheumatol 35(3):445-451

547. Chatfield SMetal (2009) Complementaryandalternative medicines in ankylosing spondylitis: a crosssectional study. Clin Rheumatol 28(2):213-217

548. Brophy S et al (2008) Internet-based randomised controlled trials for the evaluation of complementary and alternative medicines: probiotics in spondyloarthropathy. Bmc Musculoskelet Disord 9:4 
549. Jenks K et al (2010) Probiotic therapy for the treatment of spondyloarthritis: a randomized controlled trial. JRheumatol 37(10):2118-2125

550. Gotestam Skorpen C et al (2016) The EULAR points to consider for use of antirheumatic drugs before pregnancy, and during pregnancy and lactation. Ann Rheum Dis 75(5):795-810

551. Flint $J$ et al (2016) BSR and BHPR guideline on prescribing drugs in pregnancy and breastfeedingPart l: standard and biologic disease modifying anti-rheumatic drugs and corticosteroids. Rheumatol (oxford) 55(9):1693-1697

552. Villiger PM et al (2010) Effects of TNF antagonists on sperm characteristics in patients with spondyloarthritis. Ann Rheum Dis 69(10):1842-1844

553. Verstappen SM et al (2011) Anti-TNF therapies and pregnancy: outcome of 130 pregnancies in the British Society for Rheumatology Biologics Register. Ann Rheum Dis 70(5):823-826

554. Jakobsson GL et al (2016) Pregnancy outcomes in patients with ankylosing spondylitis: a nationwide register study. Ann Rheum Dis 75(10):1838-1842

555. van den Brandt S et al (2017) Risk factors for flare and treatment of disease flares during pregnancy in rheumatoid arthritis and axial spondyloarthritis patients. Arthritis Res Ther 19(1):64

556. Mahendira D et al (2014) Analysis of the effect of the oral contraceptive pill on clinical outcomes in women with ankylosing spondylitis. J Rheumatol 41(7):1344-1348

557. Huang $Z$ et al (2011) Efficacy and safety of ultrasound-guided local injections of etanercept into entheses of ankylosing spondylitis patients with refractory Achilles enthesitis. Clin Exp Rheumatol 29(4):642-649

558. Maugars $Y$ et al (1996) Assessment of the efficacy of sacroiliac corticosteroid injections in spondylarthropathies: a double-blind study. $\mathrm{Br}$ J Rheumatol 35(8):767-770

559. Luukkainen R et al (1999) Periarticular corticosteroid treatment of the sacroiliac joint in patients with seronegative spondylarthropathy. Clin Exp Rheumatol 17(1):88-90

560. Zheng Y et al (2014) Tomography-guided palisade sacroiliac joint radiofrequency neurotomy versus celecoxib for ankylosing spondylitis: a open-label, randomized, and controlled trial. Rheumatol Int 34(9):1195-1202

561. Braun J et al (1996) Computed tomography guided corticosteroid injection of the sacroiliac joint in patients with spondyloarthropathy with sacroiliitis: clinical outcome and followup by dynamic magnetic resonance imaging. J Rheumatol 23(4):659-664

562. Vander Cruyssen B et al (2010) Hip involvement in ankylosing spondylitis: epidemiology and risk factors associated with hip replacement surgery. Rheumatol (oxford) 49(1):73-81

563. Lu MC et al (2017) Incident osteoarthritis and osteoarthritis-related joint replacement surgery in patients with ankylosing spondylitis: a secondary cohort analysis of a nationwide, population-based health claims database. PLoS ONE 12(11):e187594

564. Bhan S, Eachempati KK, Malhotra R (2008) Primary cementless total hip arthroplasty for bony ankylosis in patients with ankylosing spondylitis. JArthroplasty 23(6):859-866

565. Kiaer T, Gehrchen M (2010) Transpedicular closed wedge osteotomy in ankylosing spondylitis: results of surgical treatment and prospective outcome analysis. Eur Spine J 19(1):57-64

566. Regel A et al (2017) Efficacy and safety of nonpharmacological and non-biological pharmacological treatment: a systematic literature review informing the 2016 update of the ASAS/EULAR recommendations for the management of axial spondyloarthritis. RMD Open 3(1):e397

567. Pigge RR et al (2008) Effectiveness of preoperative planning in the restoration of balance and view in ankylosing spondylitis. Neurosurg Focus 24(1):E7

568. Anwar F et al (2011) Delayed presentation and diagnosis of cervical spineinjuriesin long-standing ankylosing spondylitis. Eur Spine J 20(3):403-407

569. Hoh DJ, Khoueir P, Wang MY (2008) Management of cervical deformity in ankylosing spondylitis. Neurosurg Focus 24(1):E9

570. Sapkas G et al (2009) Surgical outcome after spina fractures in patients with ankylosing spondylitis. Bmc Musculoskelet Disord 10:96

571. Thumbikat $P$ et al (2007) Spinal cord injury in patients with ankylosing spondylitis: a 10-year review. Spine (phila Pa 1976) 32(26):2989-2995

572. Whang PG et al (2009) The management of spina injuries in patients with ankylosing spondylitis or diffuse idiopathic skeletal hyperostosis: a comparison of treatment methods and clinical outcomes. J Spinal Disord Tech 22(2):77-85

573. Krüger KAK, Rehart S, Scholz R, Kommission Pharmakotherapie der DGRh (2014) Empfehlungen der Deutschen Gesellschaft für Rheumatologie zur perioperativen Vorgehensweise unter Therapie mit DMARDs und Biologika bei entzündlichrheumatischen Erkrankungen. Deutsche Gesellschaft für Rheumatologie. https://dgrh.de/dam/ jcr:e8ac6b95-3a2f-4113-ab59-da18f1cb7cf4/ empfehlungen_periop.pdf

574. Ayhan F, Gecene M, Gündüz R (2011) Long-term effects of comprehensive inpatient rehabilitation on function and disease activity in patients with chronic rheumatoid arthritis and Ankylosing Spondylitis. Turk J Rheumatol 26:135-142

575. Staalesen Strumse YA et al (2011) Efficacy of rehabilitation for patients with ankylosing spondylitis: comparison of a four-week rehabilitation programme in a Mediterranean and a Norwegian setting. JRehabil Med 43(6):534-542

576. Masiero S et al (2011) Rehabilitation treatment in patients with ankylosing spondylitis stabilized with tumor necrosis factor inhibitor therapy: a randomized controlled trial. J Rheumatol 38(7):1335-1342

577. Van Tubergen A et al (2002) Cost effectiveness of combined spa-exercise therapy in ankylosing spondylitis: a randomized controlled trial. Arthritis Rheum 47(5):459-467

578. Mau W, Muller A (2008) Rehabilitation and outpatient physiotherapy in rheumatic disease patients. Results of cross-sectional studies of patients with rheumatoid arthritis or ankylosing spondylitis and rheumatologists. Z Rheumatol 67(7):542-553

579. Boonen A et al (2010) ASAS/WHO ICF Core Sets for ankylosing spondylitis (AS): how to classify the impact of $A S$ on functioning and health. Ann Rheum Dis 69(1):102-107

580. Boonen A et al (2009) Aspects relevant for functioning in patients with ankylosing spondylitis according to the health professionals: a Delphi study with the ICF as reference. Rheumatol (oxford) 48(8):997-1002

581. van Echteld I et al (2006) Identification of the most common problems by patients with ankylosing spondylitis using the international classification of functioning, disability and health. J Rheumato 33(12):2475-2483

582. Ward MM (1999) Health-related quality of life in ankylosing spondylitis: a survey of 175 patients. Arthritis Care Res 12(4):247-255
583. Boonen A et al (2009) Which aspects of functioning are relevant for patients with ankylosing spondylitis: results of focus group interviews. J Rheumatol 36(11):2501-2511

584. Sigl T et al (2005) ICF based comparison of disease specific instruments measuring physical functional ability in ankylosing spondylitis. Ann Rheum Dis 64(11):1576-1581

585. Gordeev VS et al (2010) Role of contextual factors in health-related quality of life in ankylosing spondylitis. Ann Rheum Dis 69(1):108-112

586. van Genderen Setal (2016) Social role participation in patients with Ankylosing Spondylitis: a crosssectional comparison with population controls. Arthritis Care Res (hoboken) 68(12):1899-1905

587. Zink A, Mau W, Schneider M (2001) Epidemiologische und sozialmedizinische Aspekte entzündlichrheumatischer Systemerkrankungen. Internist (Berl) 42(2):211-222

588. Ward MM, Kuzis S (2001) Risk factors for work disability in patients with ankylosing spondylitis. JRheumatol 28(2):315-321

589. Cakar E et al (2009) Work disability in ankylosing spondylitis: differences among working and work-disabled patients. Clin Rheumatol 28(11):1309-1314

590. Boonen A et al (2002) Work status and productivity costs due to ankylosing spondylitis: comparison of three European countries. Ann Rheum Dis 61(5):429-437

591. Boonen A et al (2001) Employment, work disability, and work days lost in patients with ankylosing spondylitis: a cross sectional study of Dutch patients. Ann Rheum Dis 60(4):353-358

592. Castillo-Ortiz JD et al (2016) Work outcome in patients with Ankylosing Spondylitis: results from a 12-year followup of an international study. Arthritis Care Res (hoboken) 68(4):544-552

593. Bönisch A et al (2005) Evaluation eines Schulungsprogramms für Patienten mit Spondylitis ankylosans. In: Pertermann F (Hrsg) Prädiktion, Verfahrensoptimierung und Kosten in der medizinischen Rehabilitation. S Roderer, Regensburg, S51-102

594. Krauth C et al (2005) Gesundheitsökonomische Evaluation eines Patientenschulungsprogramms Spondylitis ankylosans in der stationären Rehabilitation. In: Petermann F (Hrsg) Prädiktion, Verfahrensoptimierung und Kosten in der medizinischen Rehabilitation. S Roderer, Regensburg, S103-143

595. Mau W, Ehlebracht-Konig I (2013) Rehabilitation in axial spondyloarthritides. Rehabilitation (Stuttg) 52(1):51-62

596. Rodriguez-Lozano C et al (2013) Outcome of an education and home-based exercise programme for patients with ankylosing spondylitis: a nationwide randomized study. Clin Exp Rheumatol 31(5):739-748

597. Mattey DL et al (2011) Relationship between smoking and patient-reported measures of disease outcome in ankylosing spondylitis. J Rheumatol 38(12):2608-2615

598. Zhao S et al (2017) Increasing smoking intensity is associated with increased disease activity in axial spondyloarthritis. Rheumatol Int 37(2):239-244

599. Ward MM et al (2009) Clinical and immunogenetic prognostic factors for radiographic severity in ankylosing spondylitis. Arthritis Rheum 61(7):859-866

600. Dougados M et al (2011) The DESIR cohort: a 10year follow-up of early inflammatory back pain in France: study design and baseline characteristics 


\section{Leitlinien}

of the 708 recruited patients. Joint Bone Spine 78(6):598-603

601. Chung HY et al (2012) Smokers in early axial spondyloarthritis have earlier disease onset, more disease activity, inflammation and damage, and poorer function and health-related quality of life: results from the DESIR cohort. Ann Rheum Dis 71(6):809-816

602. Feldtkeller E (2002) Do patient self-help organizations help? Best Pract Res Clin Rheumatol 16(4):667-673 